\begin{abstract}
Title of Document:

A DYNAMICS-BASED FIDELITY ASSESSMENT OF PARTIAL GRAVITY GAIT SIMULATION USING UNDERWATER BODY SEGMENT BALLASTING

Adam D. Mirvis, Master of Science, 2011

Directed By:

Professor David L. Akin, Department of Aerospace Engineering

In-water testing is frequently used to simulate reduced gravity for quasi-static tasks. For dynamic motions, however, the assumption has been that drag effects invalidate any data, and in-water testing has been dismissed in favor of complex and restrictive techniques such as counterweight suspension and parabolic flight. In this study, motion-capture was used to estimate treadmill gait metrics for three environments: underwater and ballasted to $1 \mathrm{~g}$ and to $1 / 6$ th $\mathrm{g}$, and on dry land at $1 \mathrm{~g}$. Ballast was distributed anthropometrically. Motion-capture results were compared with those for a simulated dynamic walker/runner, and used to assess the effect of the in-water environment on simulation fidelity. For each test case, the model was tuned to the subject's anthropometry, and stride length, pendulum frequency, and hip displacement were computed. In-water environmental effects were found to be sufficiently quantifiable to justify using in-water testing, under certain conditions, to study partial-gravity gait dynamics.
\end{abstract}




\title{
A DYNAMICS-BASED FIDELITY ASSESSMENT OF PARTIAL GRAVITY GAIT SIMULATION USING UNDERWATER BODY SEGMENT BALLASTING
}

\author{
By \\ Adam D. Mirvis \\ Thesis submitted to the Faculty of the Graduate School of the \\ University of Maryland, College Park, in partial fulfillment \\ of the requirements for the degree of \\ Master of Science in \\ Aerospace Engineering \\ 2011
}

Advisory Committee:

Professor David L. Akin, Chair

Professor Derek Paley

Professor Norman Wereley 
(C) Copyright by

Adam D. Mirvis

2011 


\section{Acknowledgements}

A project as complex and hardware-intensive as this one would not be possible without the guidance and support of quite a few people. I first want to thank my girlfriend, Sarah Rudnick, for her infinite patience and her wonderful home-cooked meals, without which I would not have gotten through this process, and my parents, for their concern and support. I owe tremendous thanks to Max Di Capua, who has been a sounding board for ideas, a source of guidance on the thesis process, and an eager guinea pig for my admittedly harebrained-sounding test procedures. I also owe an enormous thank you to Kate McBryan, who sacrificed many hours of her time to assist with my data collection. I would like to thank Nick Limparis, Barrett Dillow, Dru Ellsberry, and Chris Carlson for their advice and expertise on all things electrical and machining-related. I would like to thank Nitin Sydney, Levi DeVries, and the rest of the Collective Dynamics and Control Laboratory for the use of their Underwater Motion Capture Facility and their OptiTrack motion capture system, and for their time and assistance in calibrating their systems and showing me how to use them. I would like to thank Dr. Dave Akin, Barrett Dillow, Ali-Abbas Husain, Sharon Singer, Nick D'Amore, and Carlos Morato for assisting with dives and performing miscellaneous manual labor (i.e., moving around my unwieldy hardware). I would like to thank the Robotics@ Maryland club for their gracious sharing of space and facilities. Finally, I would like to thank the members of my defense committee, Dr. Dave Akin, Dr. Derek Paley, and Dr. Norman Wereley, for volunteering their time and expertise. 


\section{Table of Contents}

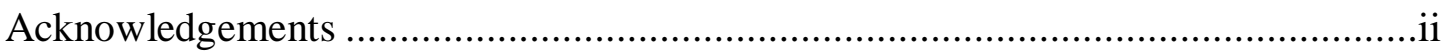

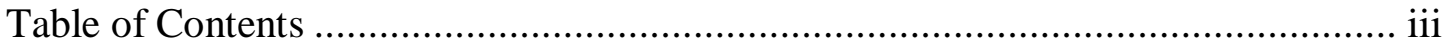

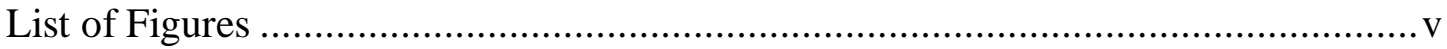

Chapter 1: Thesis Objectives and Contributions .................................................. 1

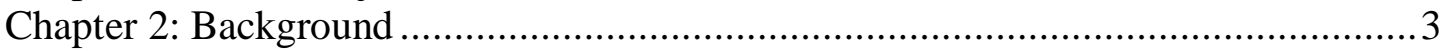

Previous reduced-gravity gait studies .............................................................

Chapter 3: Test Hardware and Equipment .........................................................6

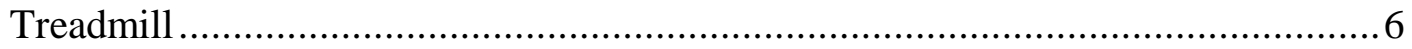

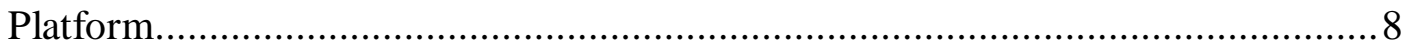

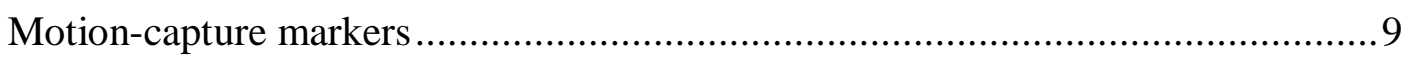

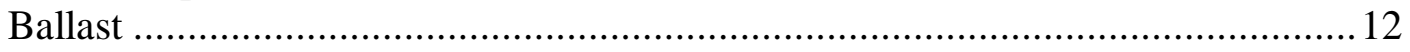

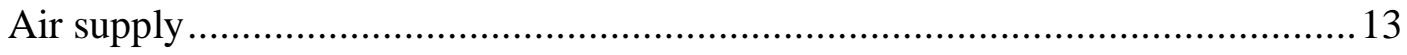

Chapter 4: Test Protocols and Procedures ……………….......................................14

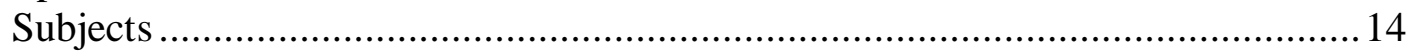

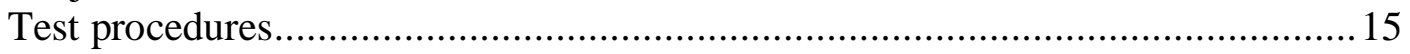

Qualitative assessment of testing process ..................................................... 20

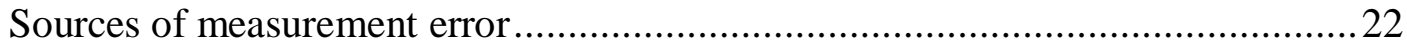

Chapter 5: Motion-Capture Data Processing ……………..................................2

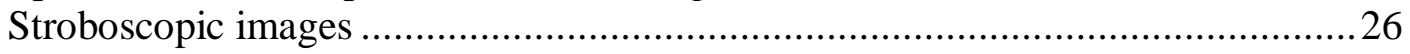

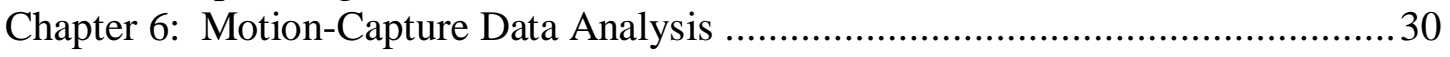

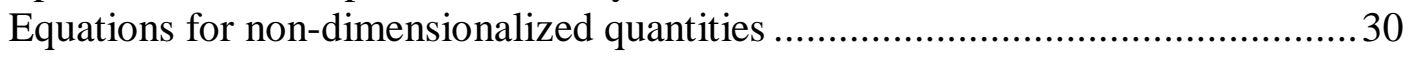

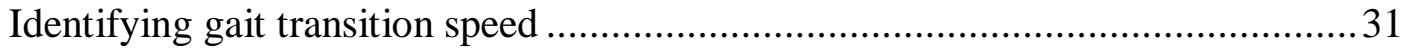

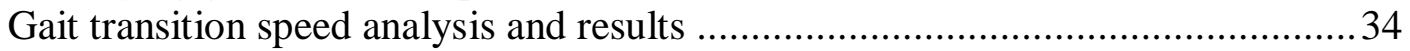

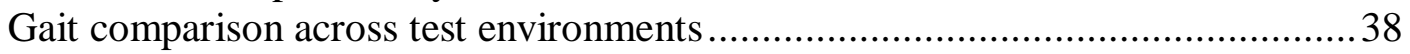

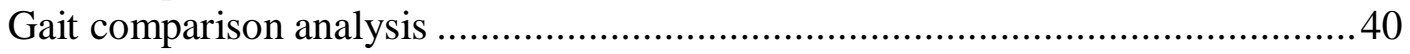

Linear regression and confidence band plots ....................................................42

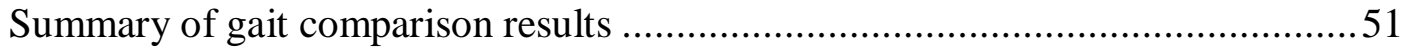

True lunar environment gait metric estimates ......................................................52

Chapter 7: Motion-Capture Testing Conclusions....................................................59

Chapter 8: Extended Test Matrix: Dynamic Walker/Runner Models ..........................61

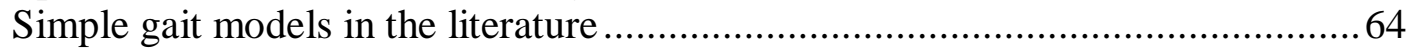

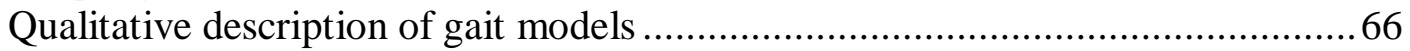

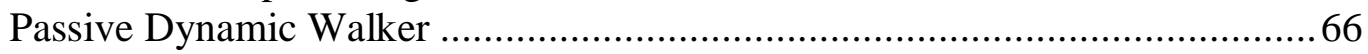

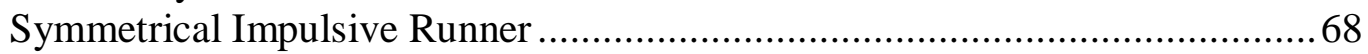

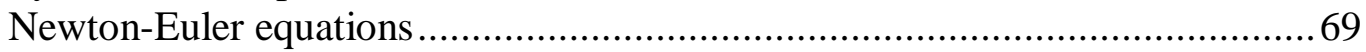

Chapter 9: Derivations of Equations of Motion for Passive Dynamic Walker............71

Definition of constants and variables ...............................................................

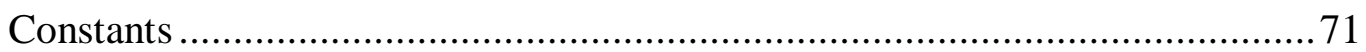

Position, velocity, and acceleration vectors for the geometric centers and centers

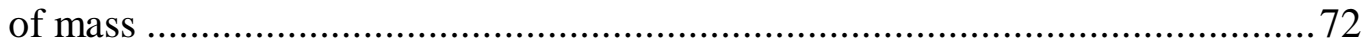

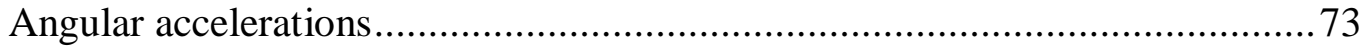

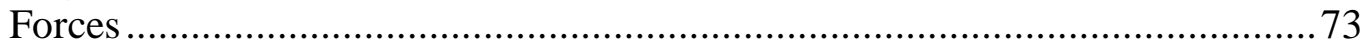




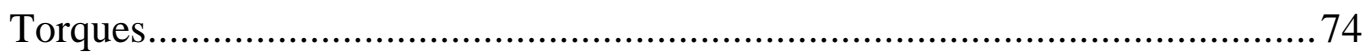

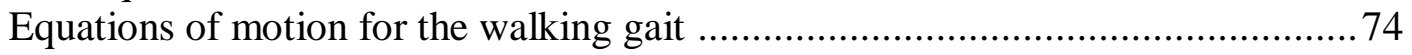

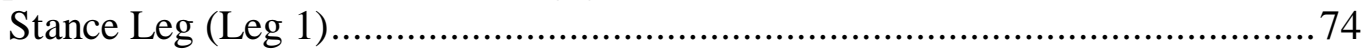

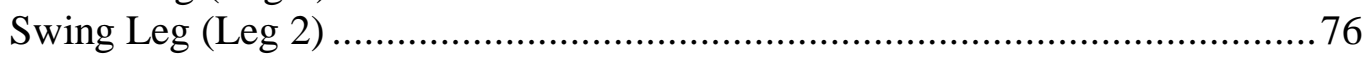

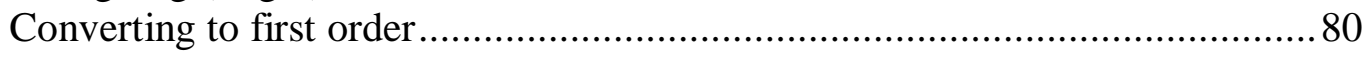

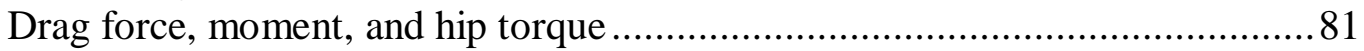

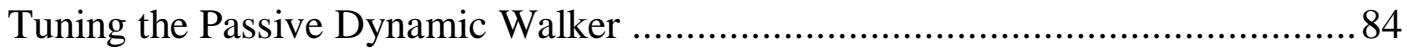

Chapter 10: Derivation of equations of motion for Symmetrical Impulsive Runner .. 89

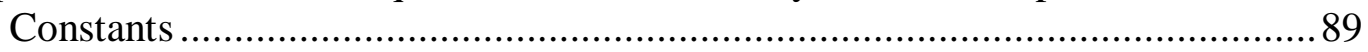

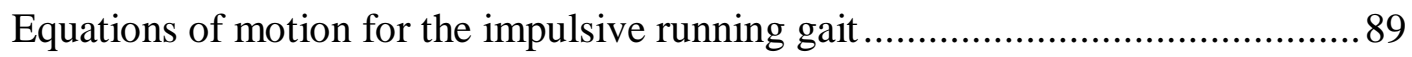

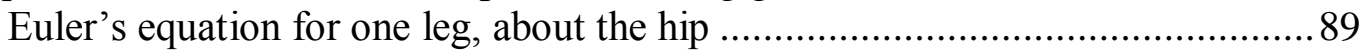

Newton's equation for the whole system .......................................................90

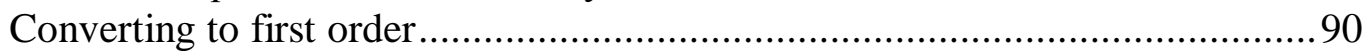

Drag force, moment, and hip torque ………………..................................

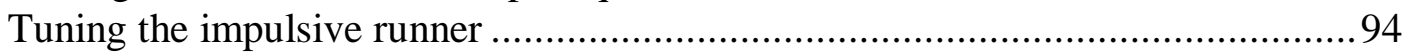

Chapter 11: Analysis of the Extended Test Matrix ..................................................96

Goal 1: Estimating gait metrics for the true lunar gravity environment .................98

Goal 2: Estimating the impact of each test environment factor on each gait metric

Virtual modeling conclusions.

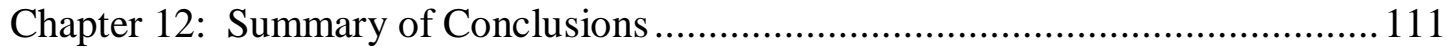

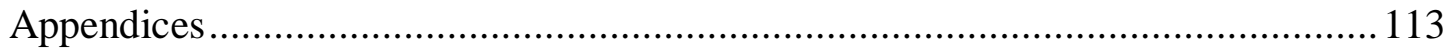

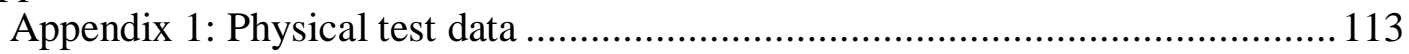

Appendix 2: Extended test matrix - linear fit plots............................................ 115

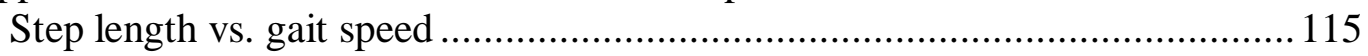

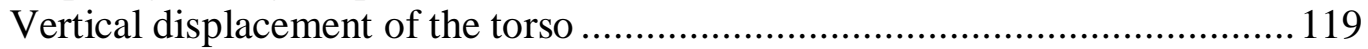

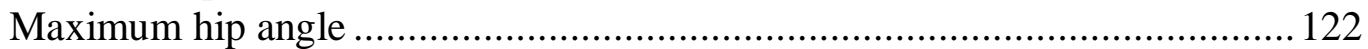

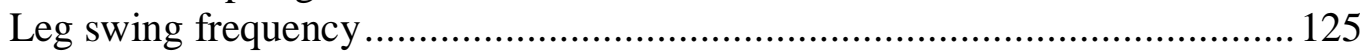

Appendix 3: Extended test matrix - difference plots ......................................... 128

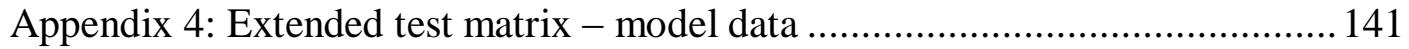

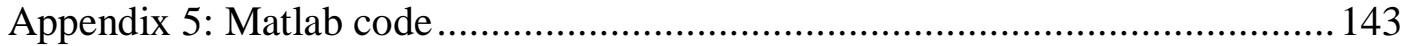

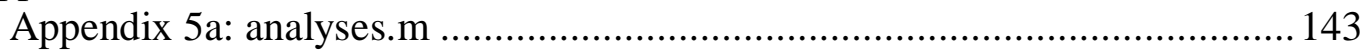

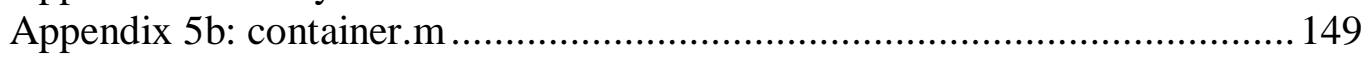

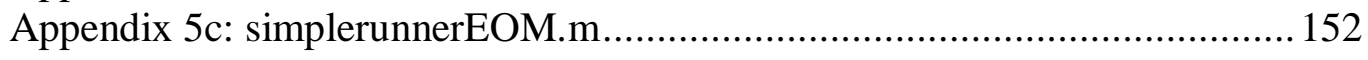

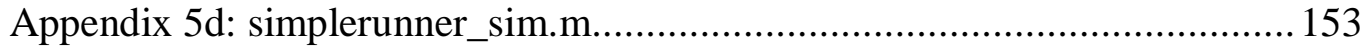

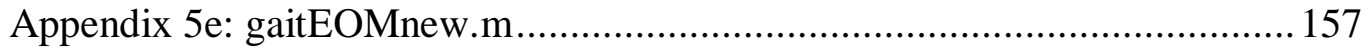

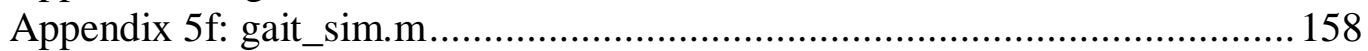

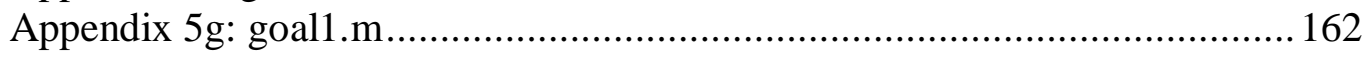

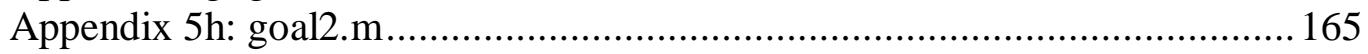

Appendix 5i: tripleimport.m.................................................................... 170

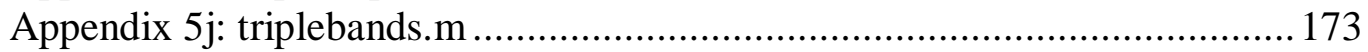

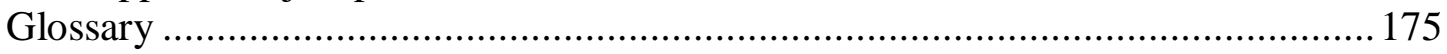

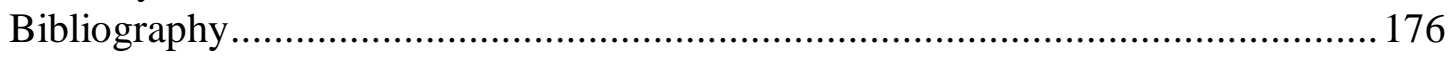




\section{List of Figures}

Figure 1: Detail of modified treadmill, showing motors, drive belt and tensioner, and

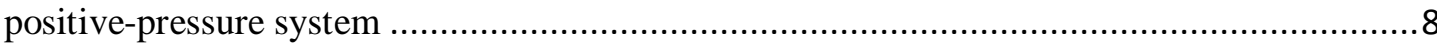

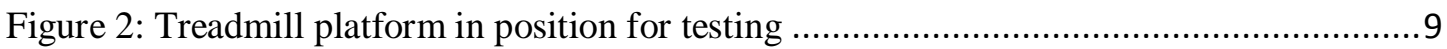

Figure 3: Approximate body positioning of motion-capture markers (Modified image from

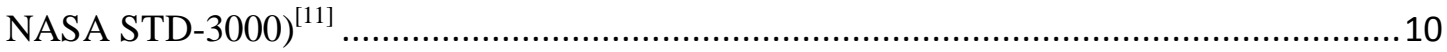

Figure 4: Detail of subject's leg, markers visible below and above the knee........................ 11

Figure 5: Detail of treadmill, showing a reflective marker .............................................. 11

Figure 6: The author walking on the treadmill while wearing the full ballast system ............13

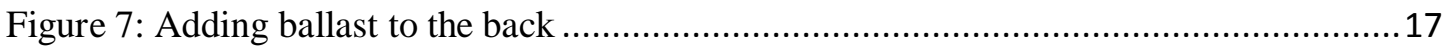

Figure 8: The treadmill being removed from the tank ................................................... 19

Figure 9: Stroboscopic image showing one step of a leg in the $1 \mathrm{~g}$, dry land test case $\ldots . . . . . . . .27$

Figure 10: "Stroboscopic" image showing step of a leg in the $1 \mathrm{~g}$, in-water test case............28

Figure 11: Stroboscopic image showing one step of a leg in the $1 / 6^{\text {th }} g$, in-water test case.. .29

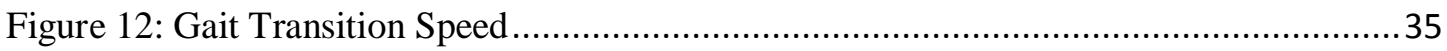

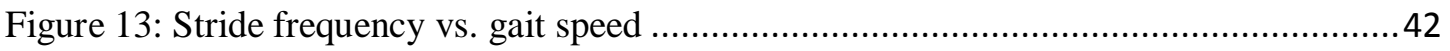

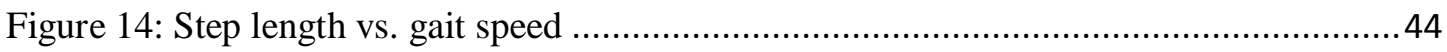

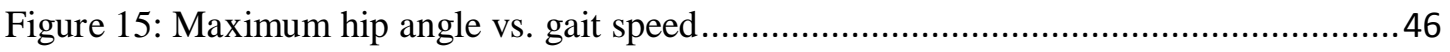

Figure 16: Vertical torso displacement vs. gait speed ........................................................... 48

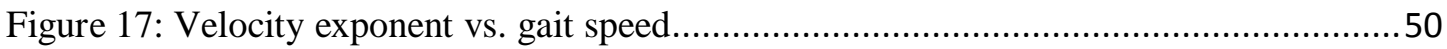

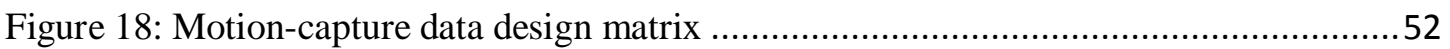

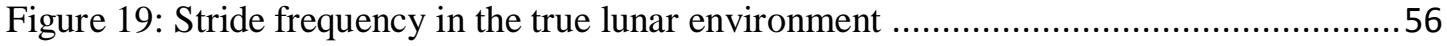

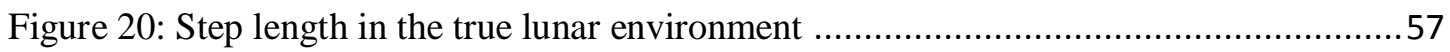

Figure 21: Maximum hip angle in the true lunar environment ...........................................5

Figure 22: Vertical displacement of the torso in the true lunar environment ........................58

Figure 23: Velocity exponent in the true lunar environment........................................... 58

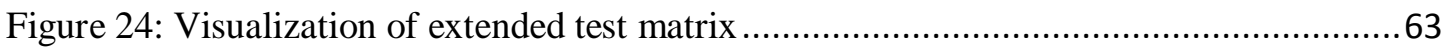

Figure 25: Definition of leg angles and coordinate frame (left) and free body diagram (right)

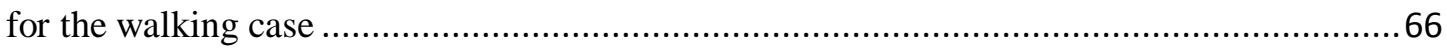

Figure 26: Definition of leg angles (left) and forces and free-body diagrams (right) .............68

Figure 27: Visualization of the test matrix used to determine gait metric functions for true

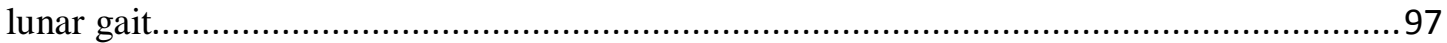

Figure 28: Step length vs. gait speed (extended test matrix) ......................................... 100

Figure 29: Vertical torso displacement vs. gait speed (extended test matrix) ......................102

Figure 30: Maximum hip angle vs. gait speed (extended test matrix) .................................103

Figure 31: Leg swing frequency vs. gait speed (extended test matrix) ..............................104

Figure 32: Modeling fidelity with regard to stride frequency ......................................... 107

Figure 33: Modeling fidelity with regard to vertical hip displacement ..............................108

Figure 34: gravity effect on step length (extended test matrix) ........................................129 
Figure 35: In-water effect on step length (extended test matrix) ..................................... 130

Figure 36: Modelling effect on step length (extended test matrix) ....................................131

Figure 37: Gravity impact on torso displacement (extended test matrix) ...........................132

Figure 38: In-water affect on torso displacement (extended test matrix) ..........................133

Figure 39: Modelling impact on torso displacement (extended test matrix) .......................134

Figure 40: gravity impact on hip angle (extended test matrix) ........................................135

Figure 41: In-water impact on hip angle (extended test matrix) ......................................136

Figure 42: Modelling impact on hip angle (extended test matrix) .................................137

Figure 43: gravity impact on swing frequency (extended test matrix) ...............................138

Figure 44: In-water impact on swing frequency (extended test matrix) ............................139

Figure 45: Modelling impact on swing frequency (extended test matrix) ..........................140 


\section{Chapter 1: Thesis Objectives and Contributions}

The overarching goal for this study was to improve understanding of environmental effects on human gait metrics, with an eye toward applicability in the planning and execution of planetary surface EVA. In particular, this study sought to better quantify, using various kinematic metrics of gait, the suitability of in-water partial-gravity ballasting and treadmill walking/running as a tool for predicting and studying gait dynamics in a true reduced-gravity environment, such as the lunar surface.

This objective was approached by means of a kinematic study, using motion-capture, of adult human gait in three environments: in the first, subjects walked on a treadmill on dry land, at normal Earth weight; in the second, the subjects walked/ran on an underwater treadmill while ballasted to $1 / 6^{\text {th }}$ of their normal weight, simulating the gravity of the lunar surface; finally, the subjects walked/ran on the underwater treadmill while ballasted to their full Earth weight. In each environment, subjects walked/ran at three progressively higher speeds, in order to examine the relationship between the non-dimensional Froude number and walk-run transition speed in these environments.

In addition to the comparisons performed between gait metrics measured in physical testing by means of motion capture, a pair of dynamic gait models were created to assess the ability of simple dynamic models to capture the behavior of corresponding 
physical environments. These models attempted to replicate various gait metrics of the real, recorded gaits.

The first-of-its-kind physical testing undertaken in this study provides a unique contribution to the field of space human factors; this study represents the first use of underwater motion capture to assess human gait dynamics in the ballasted underwater environment, with prior work relying on force measurements to estimate gait metrics. ${ }^{[14]}$ Additionally, this is the first known gait study in which subjects were ballasted underwater to a full one $g$, with prior work limited to approximately $9 / 10$ $g .{ }^{[14]}$ Given the close anthropometric distribution of the ballast, this allows, for the first time, a direct comparison of gait dynamics in the underwater and out-of-water environments, with proper gravitational force (although not inertial mass) on all relevant body segments.

The gait metrics assessed in this study allow for preliminary insights into gait energetics in reduced-gravity environments. Gait energetics in turn affect crew endurance and rates of consumables usage, key factors in EVA planning. ${ }^{[6]}$ The gait dynamics assessments conducted in this study may support future research incorporating additional measurement techniques, such as respiration measurement, to correlate gait dynamics and energetics with metabolic workload. ${ }^{[14][6][7]}$ 


\section{Chapter 2: Background}

Over the history of human spaceflight, a variety of techniques have been explored for use in simulating one or more aspects of a reduced- or zero gravity environment. Simulated reduced gravity may find use in training for spaceflight, or in studying human biomechanics or physiological response to offloading of muscles and joints. For brief periods of reduced gravity without any encumbering apparatus, parabolic flight is considered the standard of fidelity against which all other reduced-gravity simulation techniques must be compared. ${ }^{[14]}$

In addition to parabolic flight, suspension techniques are often employed, with upright, side, and supine suspension systems all having been tried. ${ }^{[15]}$ The primary limitation of suspension techniques is the inherent trade-off between mechanical complexity and simulation fidelity; simpler suspension systems apply a gravitational offset to the body mass center only, leaving the legs free to swing at their normal $1 \mathrm{~g}$ frequency. More complex suspension rigs with individual limb suspension tend to increase mechanical complexity and reduce freedom of motion to an undesirable degree. ${ }^{[14]}$

Underwater ballasting represents the third major regime in simulating reduced gravities. Use of underwater ballasting of human subjects has traditionally been limited to the study of quasi-static tasks, such as those performed by astronauts on EVA in Earth orbit. The assumption has been that drag and virtual mass effects 
induced by moving quickly though water would preempt the use of underwater ballasting for the serious study of dynamic human motions, such as gaits. However, Newman, Alexander and Webber demonstrated the use of a submerged treadmill as part of a kinetic-kinematic analysis of reduced-gravity gaits, ballasting subjects across

a range of weights from lunar to nearly Earth-normal. ${ }^{[14]}$ This project incorporates a fully kinematic analysis of underwater gaits, using motion-capture to record bodysegment positions in lieu of the split force-plate approach utilized by Newman et. al.

\section{Previous reduced-gravity gait studies}

A majority of the reduced-gravity gait studies described in the literature use some form of counterbalance rig as the means of simulating reduced gravity. Chang et al. suspended subjects in a modified climbing harness from a rolling trolley over a treadmill on a force measuring platform, with near-linear weight offset provided by a series of rubber-tubing springs. ${ }^{[2]}$ Donelan and Kram similarly used a spring-based suspension rig, opting to measure force in the suspending cable, rather than in the gait surface, and used a bicycle-seat-and-plastic-pipe assembly, straddled by the subject, to transfer the weight offset force to the subject's body. ${ }^{[3][8]}$ Perusek et al. describe a series of reduced-gravity simulators, used primarily for research into zero- $g$ exercise countermeasures. ${ }^{[15]}$

Citing the advantages of freedom of motion and unlimited simulation time, Newman, Alexander, and Webbon chose water immersion for reduced-gravity simulation. Their treadmill was powered by an electric motor outside the water, with power transferred via a flexible shaft. Subjects were outfitted with an adjustable ballast distributed 
across the chest, back, upper and lower legs, which enabled simulation of $1 / 6 g$ (lunar), ${ }^{3} / 8 g$ (Martian), $2 / 3 g$, and $9 / 10 g$ (nearly Earth-normal). Subjects traveled at 0.5 $\mathrm{m} / \mathrm{s}, 1.5 \mathrm{~m} / \mathrm{s}$, and $2.3 \mathrm{~m} / \mathrm{s}$. A split force plate beneath the treadmill and measurements of oxygen and carbon dioxide in subject's respired gases were used to assess the biomechanics and energetics of the gaits. 


\section{Chapter 3: Test Hardware and Equipment}

To create an underwater reduced-gravity environment in which one can safely and effectively assess human gait kinematics, hardware development and construction necessarily demand significant time and effort. The key hardware elements required for this study included a treadmill modified for underwater use, a 17-foot truss structure to serve as a test platform, and a ballast garment able to accommodate a wide range of subject sizes and weight requirements.

\section{$\underline{\text { Treadmill }}$}

The treadmill used in this study was a heavily-modified COTS exercise treadmill, the ProForm XP model 580S. All electronics, including the original drive motor, incline motor, motor controller board, and control console were removed. The 1.75-hp drive motor was replaced with a pair of 0.5 -hp trolling motors, designed for propelling small watercraft. The motors each contributed a portion of the torque demanded, and transferred power to the tread via a tensioned rubber drive belt. The motor drive wheels and drive belt tensioner mechanism were machined and assembled in-house.

Two readily-available trolling motors were used to drive the treadmill, due to the cost of obtaining a single, sufficiently powerful motor designed for underwater operation. Although the two-motor system ultimately performed as desired, its implementation created a challenge. Due to age, wear, and manufacturing variability, the two motors 
were not identical in performance, and spun at different speeds when the same voltage was applied across them. However, by wiring the motors in series, and tensioning the drive belt sufficiently so as to minimize slippage, the motors were forced to spin at the same speed, by drawing slightly different voltages. Wiring the motors in series had the secondary advantage of minimizing the amount of current which would be sent into the tank, a key safety consideration.

An Agilent model 6032 DC power supply provided 30 volts across the motors, at a maximum current of 25 amps. The power supply maintained a constant voltage, and allowed current to vary in response to the demand placed on the motors. Thus, although the torque applied to the motors varied with subject mass and across different phases of each stride, the treadmill was able to spin at a constant speed throughout the stride and across subjects for each test condition. For safety and convenience, a simple relay circuit was installed which allowed the test director or the subject to turn the treadmill on or off using a switch mounted to the treadmill handlebar. A COTS transformer converted grid AC to 12 volt DC to operate the relay circuit.

To prevent water from leaking in and shorting the motors, a positive pressure system was constructed. Whenever the treadmill was to be immersed in water, the motors housings were connected via a shop air hose to an air compressor at the SSL facility. An adjustable regulator mounted on the treadmill maintained a pressure in the motor casings of 3-5 psi above ambient. 
To accommodate test subjects, the treadmill handlebar was padded for safety, and nylon straps were attached at the side of the treadmill to secure the subject's air tank.

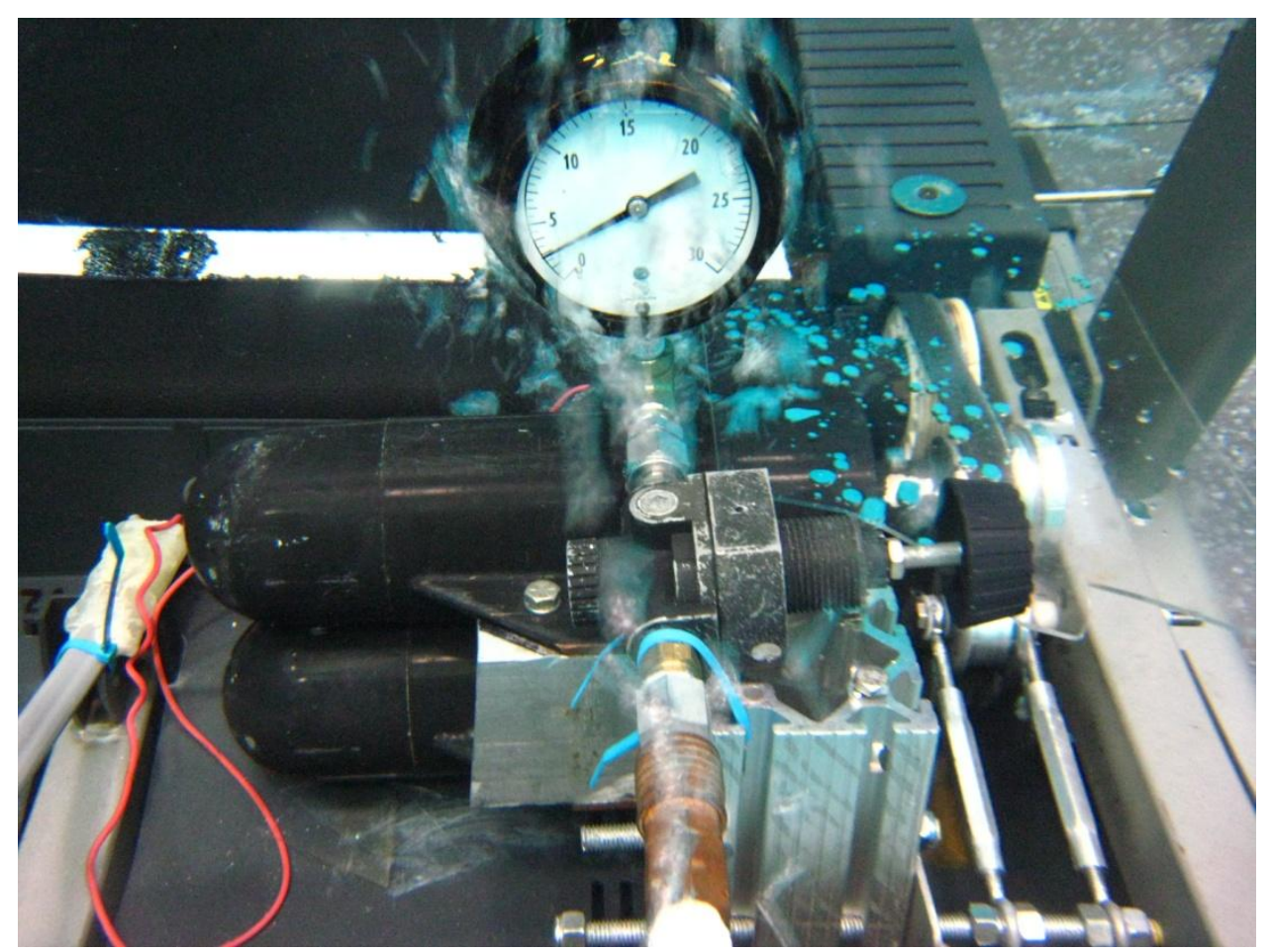

Figure 1: Detail of modified treadmill, showing motors, drive belt and tensioner, and positivepressure system

\section{Platform}

In order for the treadmill and subject to be within the field of view of the motioncapture cameras, the treadmill had to be located in the upper half of the tank, roughly centered relative to the walls of the tank. This required the construction of a stationary platform to support the treadmill and subject at this location. A 16-foot, 7inch tall by 6-foot by 6-foot truss was constructed from fiberglass I-beams, with 
nylon rope and ratcheting die-down straps serving as additional tensional members to increase the rigidity of the structure. The truss was secured to four hard points around the perimeter of the base of the tank using rope and ratchet straps. A $1 / 4$ "-inch thick aluminum plate, secured with C-clamps to the top of the truss structure, served as the deck for the treadmill.

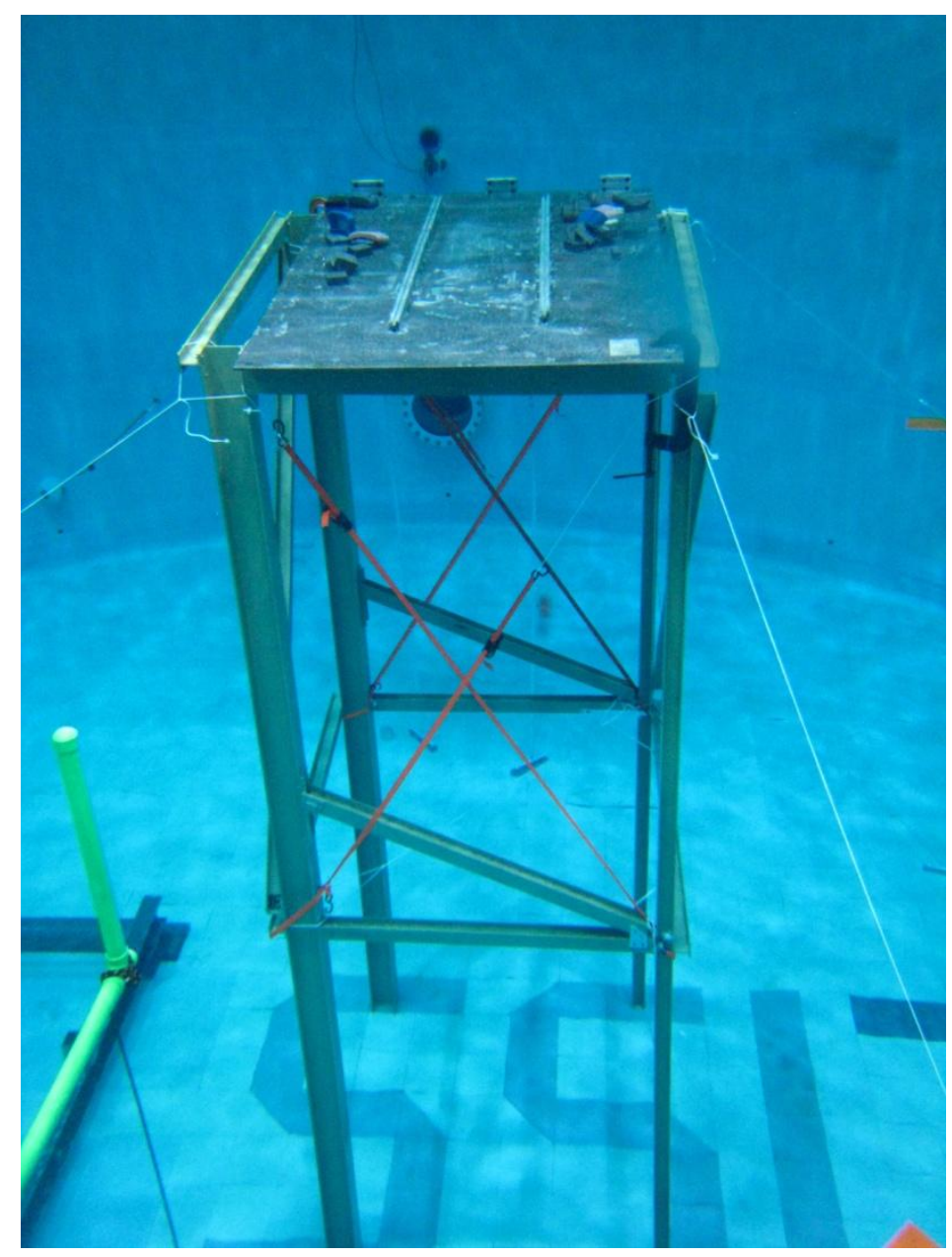

Figure 2: Treadmill platform in position for testing

\section{Motion-capture markers}

A total of twelve 33-mm-diameter motion-capture markers were used during data collection: four markers were mounted rigidly to the corners of the treadmill, to track 
undesirable motion of the platform; one marker was mounted on each hip at the protrusion of the greater trochanter ${ }^{[4]}$; one marker was mounted on each thigh just above the knee; one marker was mounted on each calf just below the knee, and one marker was mounted on each ankle. All six markers worn on the subjects' legs were located along the outside of the leg, in the coronal plane. The eight body markers were mounted to adjustable fabric straps that were used to hold and position the markers on each subject's body. Several subjects opted to wear a loose-fitting coverall for comfort, as the marker straps on bare skin were found to have a tendency to pull at leg hairs.
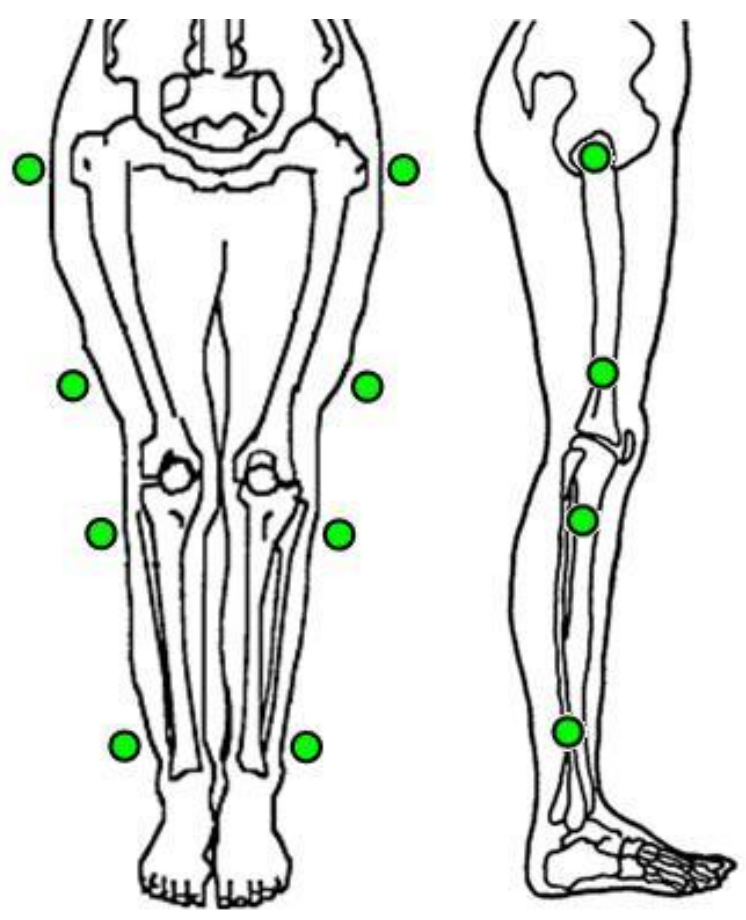

Figure 3: Approximate body positioning of motion-capture markers (Modified image from NASA STD-3000) ${ }^{[11]}$ 


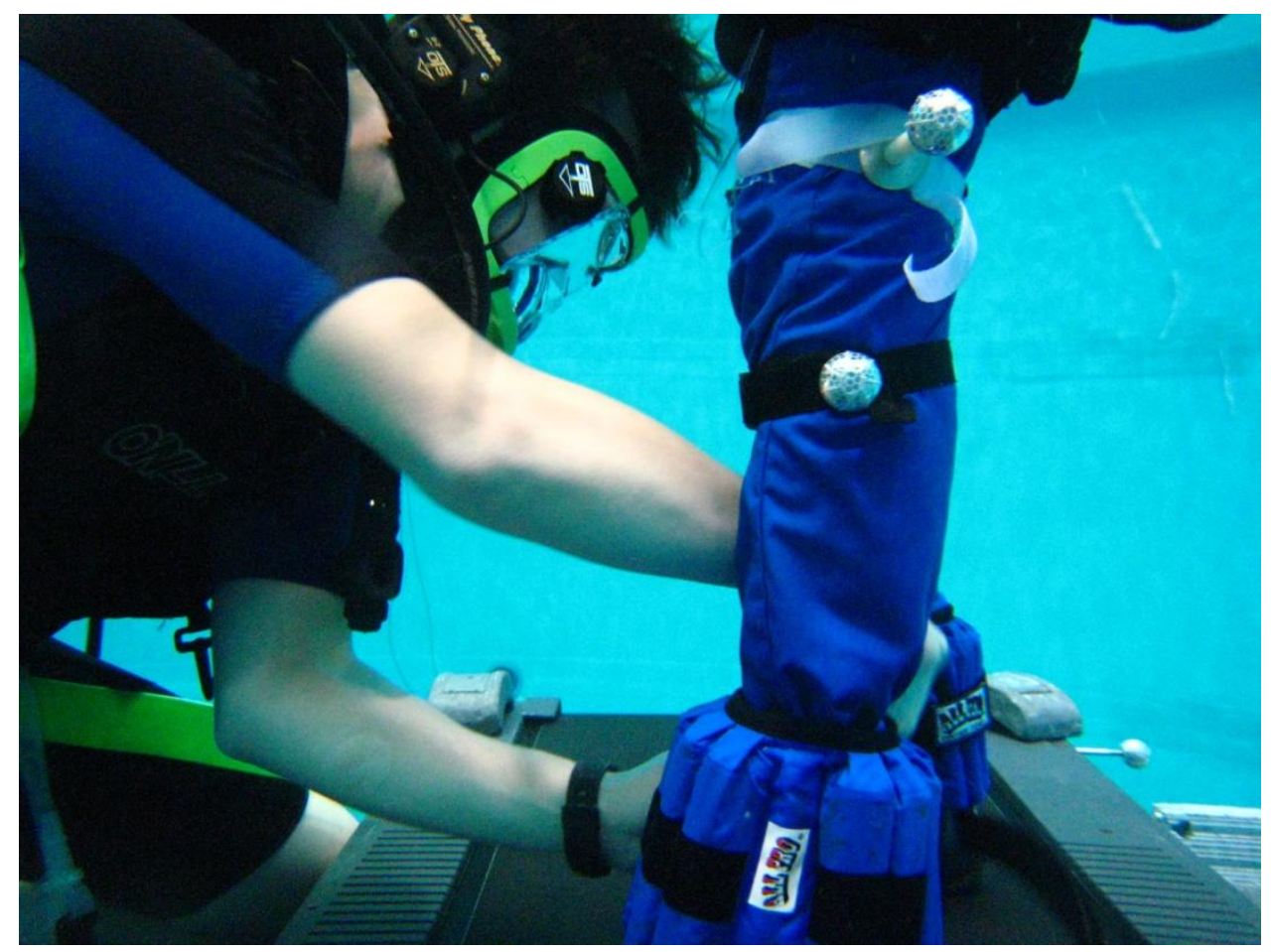

Figure 4: Detail of subject's leg, markers visible below and above the knee

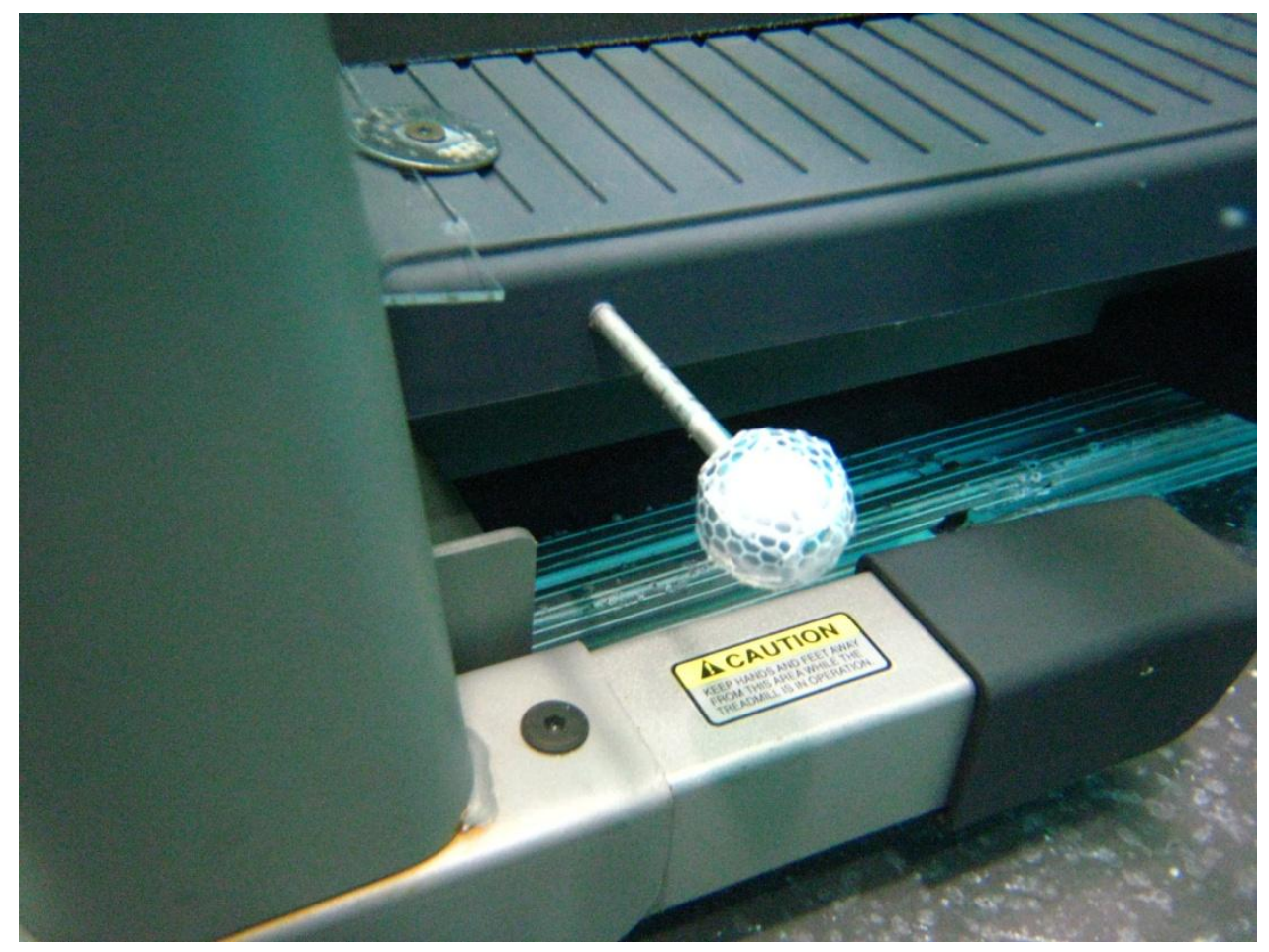

Figure 5: Detail of treadmill, showing a reflective marker 


\section{$\underline{\text { Ballast }}$}

The design of the ballasting system was driven by the extreme case of high subject mass and high simulated gravitational load. A maximum body mass of $200 \mathrm{lbs}$. was set as a requirement for test subjects, to allow the ballasting hardware to remain reasonably easy to assemble, disassemble, don, and doff. The ballast system was designed such that weight could easily be added or removed to alternate between the $1 / 6^{\text {th }} g$ and $1 g$ test cases.

A distributed ballast system was selected over a torso-only system to more accurately represent the distribution of gravitational forces over the walking body, with ballast located on the front of the torso, the back of the torso, the thighs, and the calves. An appropriate ballast distribution was calculated using body segment mass data for $50^{\text {th }}$ percentile American males. ${ }^{[11]}$ This resulted in placing $62 \%$ of the ballast in a given case on the torso (split evenly into $31 \%$ on the chest and $31 \%$ on the back), $13 \%$ on each thigh, and $6 \%$ on each calf. For the heaviest subject in the $1 g$ test case, this corresponded to $62 \mathrm{lbs}$. of ballast on the front of the torso, $62 \mathrm{lbs}$. on the back of the torso, $26 \mathrm{lbs}$. on each thigh, and $12 \mathrm{lbs}$. on each ankle.

The ballast system was assembled entirely from COTS components, primarily modular elements of a military tactical gear system which assembled with interweaving straps and snaps. The torso unit consisted of a vest, with a large pack on the back and several smaller ammunition pouches on the chest and sides. Two "drop 
leg" pouches strapped around the thighs and attached to a waist belt. A pair of COTS ankle weight belts completed the ballast system.

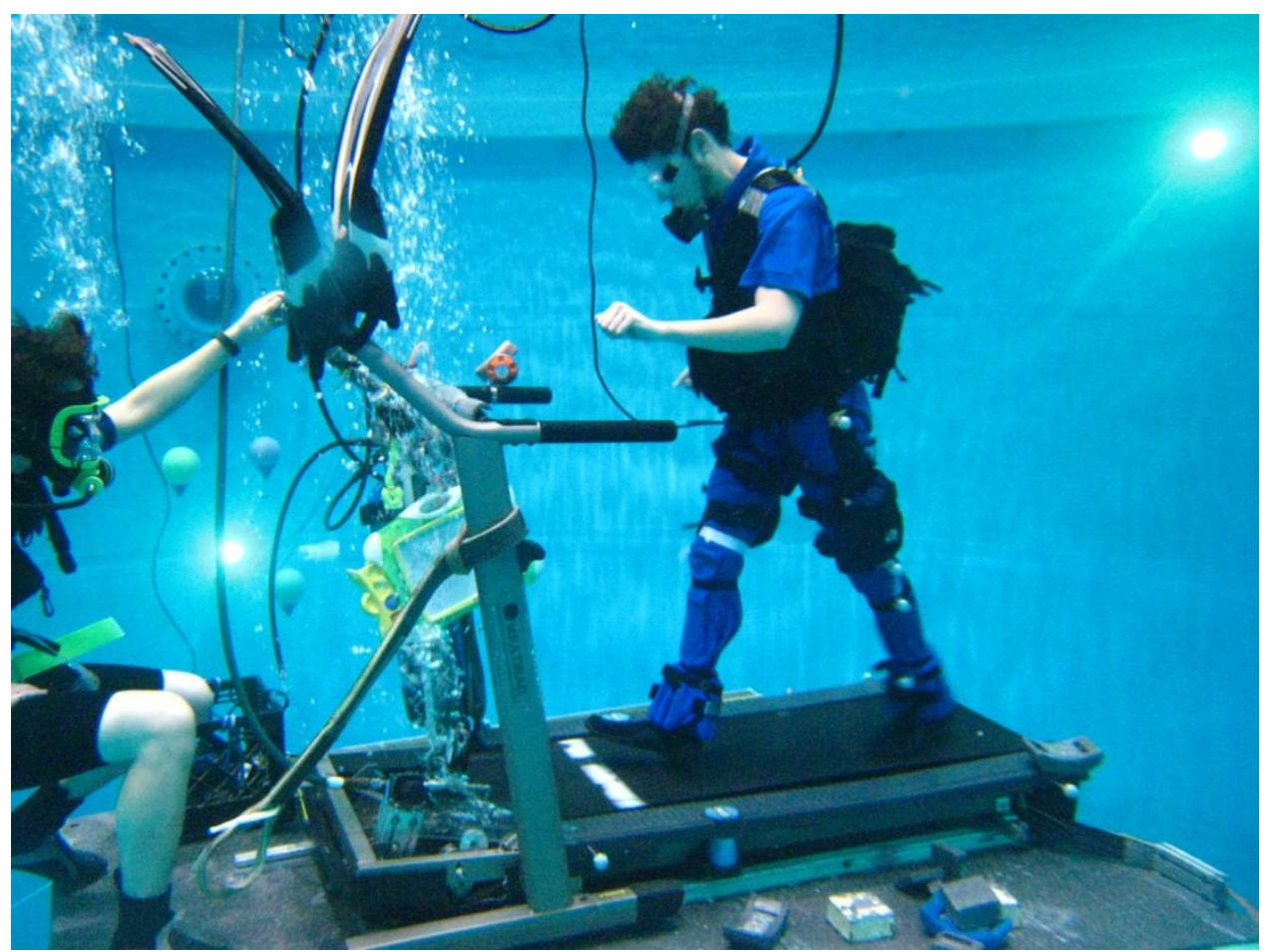

Figure 6: The author walking on the treadmill while wearing the full ballast system

For safety, test subjects wore a standard climbing harness, attached via a slack rope to an overhead crane. In the event a subject were to fall off the treadmill and platform while ballasted, this would ensure that they did not sink to the bottom of the tank.

\section{Air supply}

During in-water testing, subjects breathed from a standard scuba bottle mounted to the treadmill, via a "hookah" rig, a regulator with an extra-long hose to allow the subject freedom of motion. 


\section{Chapter 4: Test Protocols and Procedures}

Subjects in this study participated in two test sessions, one in the water and one out of the water. In-water testing took place at the University of Maryland Space Systems Laboratory's Neutral Buoyancy Research Facility, using the Underwater Motion Capture Facility (UMCF) owned and operated by the Collective Dynamics and Control Laboratory (CDCL). Dry-land testing took place in the Manufacturing Building on the UMd campus, using a second motion-capture system also belonging to the CDCL.

\section{$\underline{\text { Subjects }}$}

Five subjects, four male and one female, participated in the testing. All subjects were between the ages of 24 and 30 . The mean subject body weight was $74.8 \pm 14 \mathrm{~kg}$. The

mean leg length, as measured from the floor to the greater trochanter of the femur ${ }^{[4]}$ while standing with shoes off, was $90.7 \pm 5 \mathrm{~cm}$.

All subjects were PADI- or NAUI- certified scuba divers previously approved to participate in dive operations at the NBRF. All subjects had normal (unimpeded) gaits, and reported no medical conditions which would preclude participation or invalidate the collected data. Each subject was briefed on the test procedures and completed a University-approved informed consent agreement prior to testing. 


\section{Test procedures}

During the in-water session, subjects walked/ran on an underwater treadmill at three progressively greater speeds, first while ballasted to $1 / 6^{\text {th }}$ of their normal weight, and again while ballasted to their full normal weight, while the array of motion-capture cameras recorded the position of markers on their hips and legs at $20 \mathrm{~Hz}$. Each run lasted approximately 45 seconds, so that roughly 30 gait cycles (60 steps) were captured. In addition to the motion-capture data, still photographs and video were recorded during each dive.

While on the treadmill underwater, subjects breathed using a long "hookah" rig connected to a scuba tank mounted to the treadmill. Subjects were not permitted to wear a wetsuit during the in-water testing, as the buoyancy and range-of-motion restriction of a wetsuit could potentially alter the test results.

Procedures for the in-water sessions are as follows. Before getting in the water, subjects were weighed and key anthropometric dimensions (knee height, hip height) were measured. The subjects' weight was used to compute ballast loads for the torso, thighs, and calves for the $1 g$ and $1 / 6^{\text {th }} g$ test segments. The subjects then changed into the empty ballast garment and climbing harness, assisted by the student investigator as necessary, and donned the reflective markers used by the motioncapture system. 
In addition to the test subject and the student investigator, a third diver participated in each session as a safety diver, with the sole responsibility of watching, and, if necessary, assisting the test subject. This diver was equipped with a full facemask to allow communication with the surface. Before each subject entered the tank, the treadmill was lowered by crane onto its platform, and the student investigator and secondary diver prepared it for use.

Upon entering the water, subjects swam to the treadmill platform, removed their fins, and switched from their personal scuba tank to the one on the treadmill. They were then secured via their harness to a slack rope mounted to an overhead crane, which served as a safety measure in case a subject were to fall off of the platform while ballasted.

Based on the ballast loads computed earlier, the student investigator then strapped the adjusted ankle ballasts onto the subject, and proceeded to load the remaining pockets of the ballast garment with lead weights, working from thighs to chest and sides to back. During the ballasting process, the investigator periodically directed the subject to stand on a spring scale so that the ballast could be checked and adjusted accordingly. This process took approximately 5-10 minutes. 


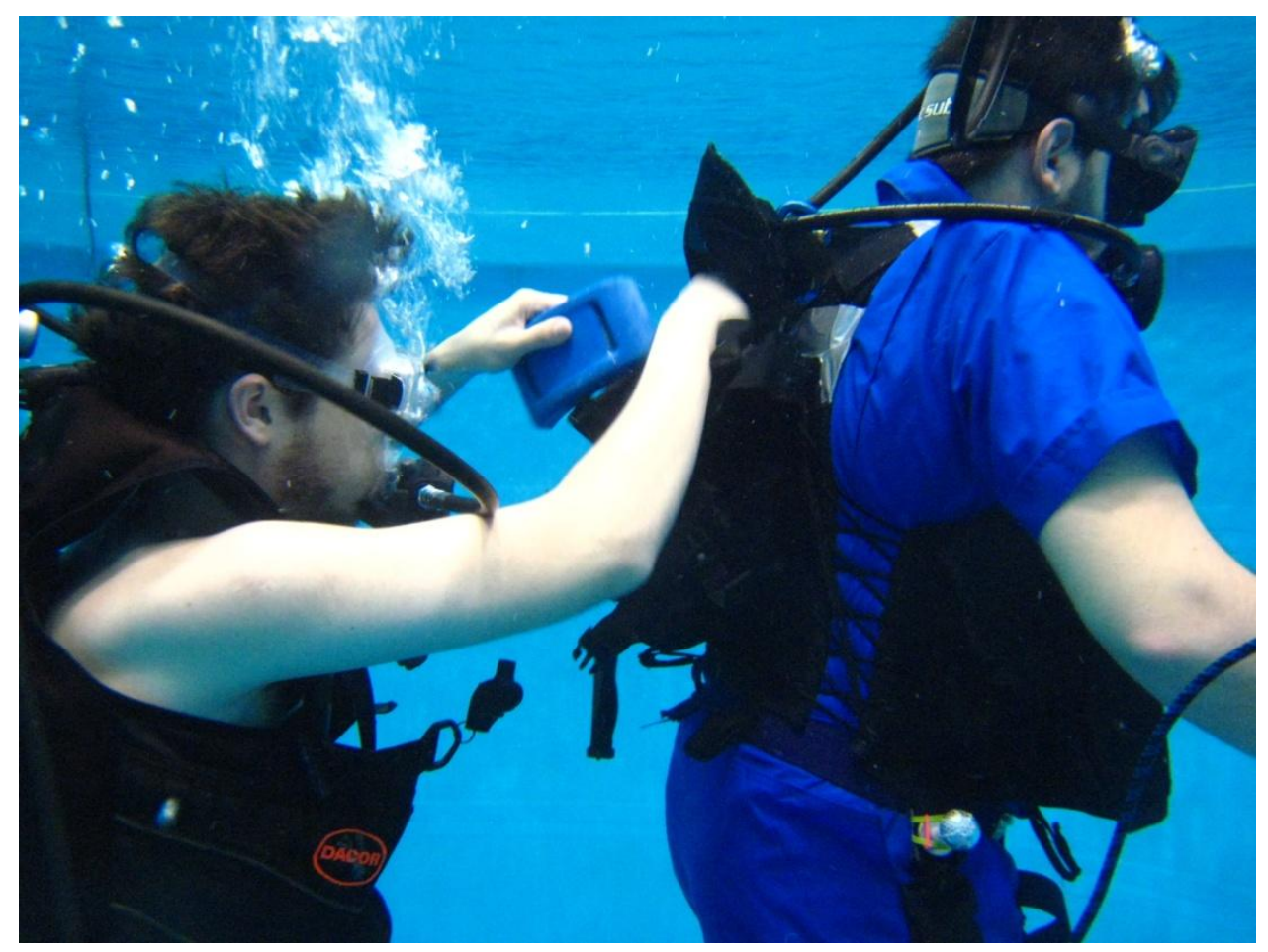

Figure 7: Adding ballast to the back

Once a subject was fully ballasted, the investigator would direct the deck chief to turn on the treadmill power supply and set it to output up to 25 amps at 15 volts, and direct the data collection assistant to prepare for a motion-capture run. The subject wound then flip on the treadmill kill switch when ready, and began walking/running. After a 45 second motion-capture run, the investigator would direct the subject to stop the treadmill and wait for the next run. The intestigator would then direct the deck chief to adjust the power supply to 25 amps and 20 volts. A second run would be performed at 20 volts and a third at 25 volts.

After the three runs at $1 / 6^{\text {th }} g$, the investigator would add additional ballast to all pockets, again working from ankles to thighs to thighs to torso and checking 
periodically with the spring scale, until the subject was ballasted to their full normal weight with mass distributed anthropometrically. With the subject ready, data collection runs were again performed at 15,20 , and 25 volts.

At the conclusion of the last run, the investigator would direct the deck chief to cut all power to the treadmill, and would begin removing weight from the subject's ballast garment, in the opposite order of how it was put in. The unburdened subject would then switch back to their personal scuba tank, don their fins, swim to the diving platform, and exit the tank. The investigator and secondary diver would then clean up the test platform, re-attaching the treadmill to the crane for removal from the tank, and returning the spring scale and fabric ballast bags to the surface so as to forestall corrosion and disintegration. 


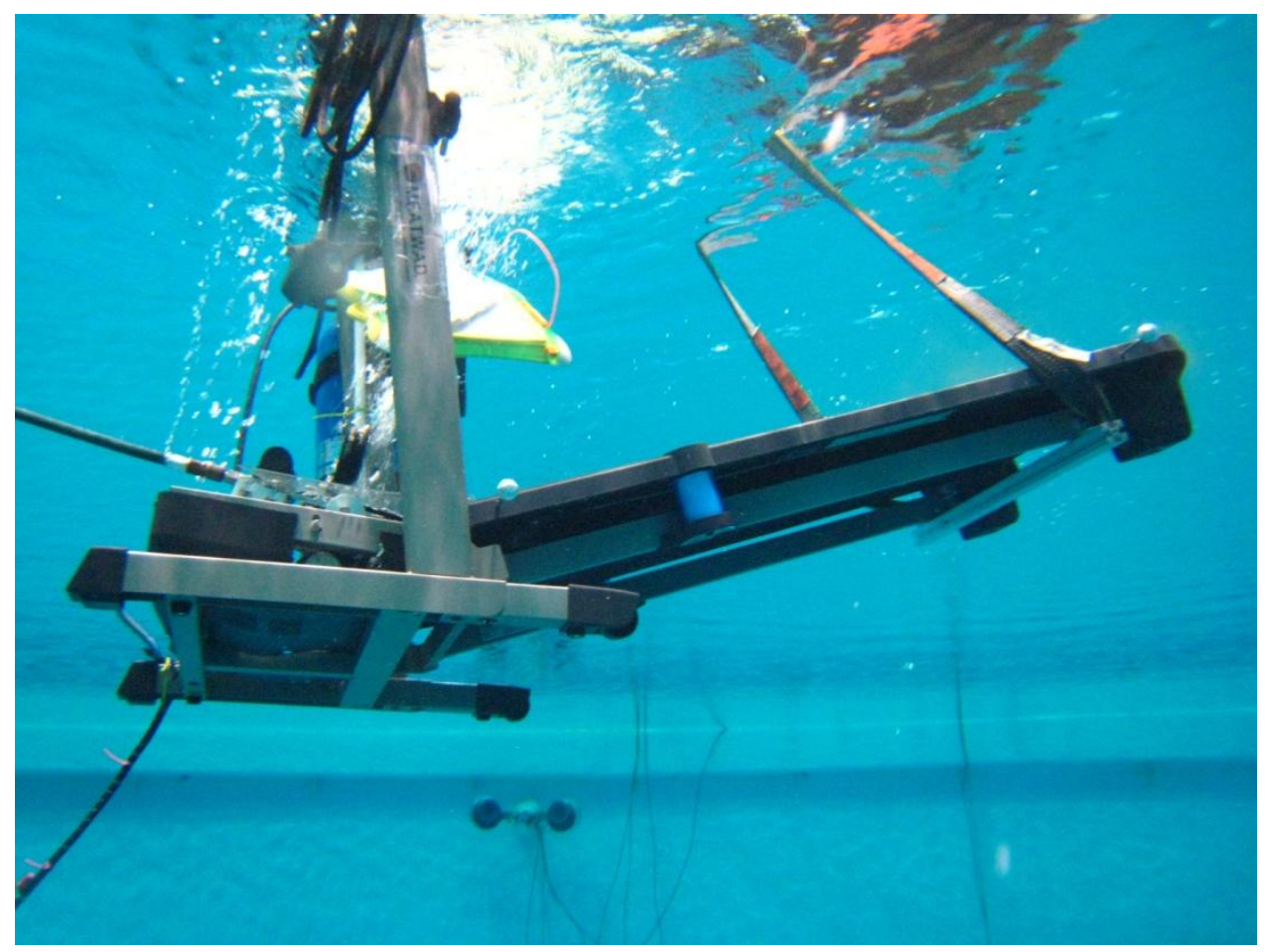

Figure 8: The treadmill being removed from the tank

The dry-land test sessions were far simpler and quicker than the in-water sessions, requiring no more than about 10 minutes of each subject's time, compared with the hour to an hour and a half required for the in-water sessions, even neglecting prep work. The subjects simply donned the reflectors, got on the treadmill, and performed runs at 15,20 , and 25 volts.

At the end of testing, subjects were asked to complete a short post-questionnaire about their experiences. The results of these questionnaires are discussed below. 


\section{Qualitative assessment of testing process}

A total of five subjects participated in testing, with all five subjects completing all test sessions. Feedback from the subjects primarily concerned the in-water sessions.

In the post-questionnaire, subjects were asked what, if any, difficulties they encountered during testing. Several commented that, while moving at $1 / 6^{\text {th }} g$ was not exhausting, it was difficult to retain one's balance. Multiple subjects commented that

moving at $1 \mathrm{~g}$ was more difficult than $1 / 6^{\text {th }} g$, but disagreed on whether slower or faster runs at $1 g$ were harder.

Subjects were also asked what, if any, discomfort they experienced during testing. Most subjects reported some discomfort in the back and shoulders associated with carrying the ballast for the $1 \mathrm{~g}$ runs, and one subject commented that the duration of the $1 g$ portion was an important factor in discomfort level. One subject reported a weight digging into their lower back. In addition, three subjects reported some discomfort or exhaustion due to movement of the thigh ballasts during gait.

Additional comments made by subjects included that tight straps were important to preventing shifting of the thigh ballasts; that the movement of the treadmill support structure could be perceived at $1 \mathrm{~g}$; that it took some time to adjust to new gaits, but that gait became more natural towards the ends of runs; that a flag mounted to the front of the treadmill served as a useful visual reference point for maintaining balance, and that the strobe-light effect created by the motion-capture cameras while 
operating was a distraction but that one got used to it. Two subjects commented that the $1 / 6^{\text {th }} g$ portion was "really fun".

From the perspective of the student investigator, the testing process carried a sharp learning curve, with unanticipated issues plaguing an early test run, but with each subsequent session becoming easier and taking less time. In particular, the ballasting process became significantly more efficient over the series of sessions. With the same individuals assisting as safety diver, deck chief, and data collection assistant for multiple sessions, communicating the steps of the test procedure became easier with familiarity.

One key logistic concern for this sort of testing is manpower. Each in-water session involved a subject, an investigator, a safety diver, a deck chief, and a data collection assistant, with each role requiring specialized knowledge and/or status. For safety reasons, each of the above personnel (with the exception of the data collection assistant) had to be certified scuba divers specifically approved to dive at the NBRF, with CPR/AED/first aid certifications up to date. Given the limited number of people meeting these criteria, and the hectic schedules of graduate students, it should be noted that determining manpower requirements early is a crucial step in the design of any human subject research project involving scuba diving. 


\section{Sources of measurement error}

There are several potential sources of error in the physical measurements obtained during testing. Error in measured subject weight due to miscalibration of the spring scale and parallax induced by the viewing angle of the scale is estimated to be approximately $\pm 1 \mathrm{~kg}$. Error in measured body-segment lengths is estimated to be $\pm 3 \%$. Imprecise placement and shifting of markers on the body is estimated to contribute an error of $\pm 3 \mathrm{~cm}$ in each axis to the measured marker positions throughout each run. Position drift over each run due to motion of the treadmill platform is estimated to be $\pm 2 \mathrm{~cm}$ in the front-back axis and $\pm 1 \mathrm{~cm}$ in the left-right axis, affecting all the body markers equally.

The motion-capture system itself introduced several sources of error which are difficult to quantify, but which are assumed to be relatively minor contributors to overall measurement error. Calibration of the system may introduce position drift of approximately $\pm 0.03 \mathrm{~cm}$ in each axis over the course of a run, affecting all markers equally. This error is estimated from the variation in the recorded position of the fixed treadmill markers over the course of each run using the dry-land motion-capture system, and is much smaller than the error introduced in the underwater data by motion of the treadmill platform. Slight position errors may be introduced by the gapfilling algorithm used by the Qualisys software, though this source of error is much smaller than the other sources of error in recorded marker position, and is present for only a small percentage of each recorded marker trajectory. 
Finally, position errors may be introduced into in the data-processing stage by mislabeling of marker trajectory segments and inclusion of false positive trajectory segments, such as those introduced by bubbles and reflections. In processing the underwater data, trajectory segments were labeled and false positives pruned by visual inspection. For the dry-land data, the process of trajectory identification was automated in MATLAB, and visual inspection was used to confirm correct labeling and absence of false positives. For both the underwater and dry-land data, it is believed that all trajectory segments were correctly labeled and all false positives removed. 


\section{Chapter 5: Motion-Capture Data Processing}

Body-segment position data for the in-water and out-of-water sessions were obtained using two different motion-capture systems, each with its own proprietary software package, and thus required different methods and levels of processing to yield useful gait metrics. First, the cloud of points generated by the motion capture systems had to be turned into continuous marker trajectories, and false positives eliminated. The Qualisys system in use at the UMCF automatically stitches together many broken trajectories, and includes an intuitive GUI for manual data cleanup. Bubbles from the divers' regulators and the air hose feeding the motors were a source of many false positives, but these were easily visually distinguished from the marker trajectories.

The OptiTrack system in use in the Manufacturing building is designed primarily for tracking definable rigid bodies, and is ill-equipped to track isolated markers. It outputs a tab-separated-values file with rows containing the positions of markers visible at each frame. Markers which disappear, even for a single frame, reappear at the end of the list, with the intervening markers shifting up one space. In order to pull out continuous marker trajectories from this data, the markers in each frame were sorted and identified by their relative positions, with the highest two markers representing the hips and so forth (see MATLAB code in Appendix 5a). All data processing was performed on an HP Pavilion ${ }^{\circledR}$ dv5 notebook PC with an Intel ${ }^{\circledR}$ Core $^{\mathrm{TM}} 2$ Duo CPU running at $2.4 \mathrm{GHz}$ and $3.0 \mathrm{~Gb}$ of RAM, on Windows Vista ${ }^{\mathrm{TM}}$ Home Premium. 
Once the raw data from each motion-capture system was sorted into continuous marker trajectories, virtual markers were created for each run from the recorded marker trajectories and known subject anthropometry. A virtual torso marker was created at the center of the two hip markers, so that vertical motion of the trunk during gait could be assessed without being affected by rotation of the pelvis in the coronal plane. Virtual heels were created along the line defined by the below-knee and ankle markers, at a distance from the ankle marker such that at its lowest point in the stride, the height of each heel is precisely at the height of the walking surface, as determined from the four stationary markers mounted to the corners of the treadmill. Virtual knee markers were created along the same line, at a distance from the heels determined by the measured standing knee height of each subject. Creating virtual heel and knee markers from known geometry obviated the need for precise placement of markers along the length of the calf and at the knee itself.

With virtual markers in hand, gait metrics were computed for each step, as defined by the intervals between cross-over of the heels. Step duration, speed, step length, vertical displacement of the torso, maximum horizontal distance between the heels, and maximum angle between the legs in the sagittal plane were computed from marker positions, and averages were taken over the length of each run (See MATLAB code in Appendix 5b). 
In addition, a metric "isrun" was created to assess whether each individual step seemed to qualify as a run, based on the height both feet come off the ground. Averaged over each run and across all subjects, this metric essentially reveals the percentage of a run that resembled running rather than walking. Looking across the test cases, it shows an abrupt transition: for $1 \mathrm{~g}$ runs on dry land and at low speed in water, "isrun" is near zero; for $1 / 6^{\text {th }} g$ runs and $1 g$ runs in water at high speed, "isrun" is greater than 0.85 . For medium speed, $1 \mathrm{~g}$ runs in water, isrun $=0.493$, indicating that this case was approached with a walking-type gait and a running-type gait in nearly equal proportions.

The full set of gait metric data obtained through physical testing can be found in Appendix 1.

\section{Stroboscopic images}

Using the virtual markers created during processing of the motion capture data, a series of "stroboscopic" images may be created, showing the joint positions and angles at various points in time over a gait cycle. These images may be useful for getting an intuitive sense of the dynamics of a gait. Stroboscopic images for the three motion-capture test environments are presented and briefly discussed below. 


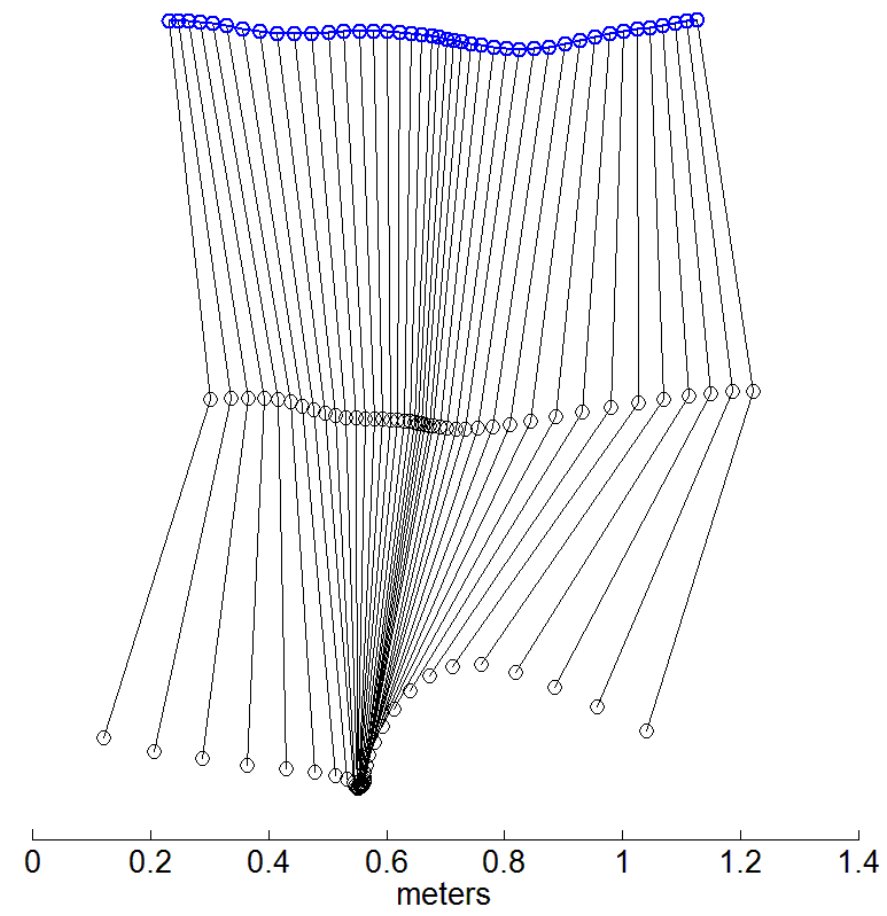

Figure 9: Stroboscopic image showing one step of a leg in the $1 g$, dry land test case

The first stroboscopic image presents a typical gait cycle, for one leg, in the dry, $1 \mathrm{~g}$ environment. Note that vertical displacement of the hip is small, that the leg does not extend very far beyond vertical, and that the knee bend is small. 


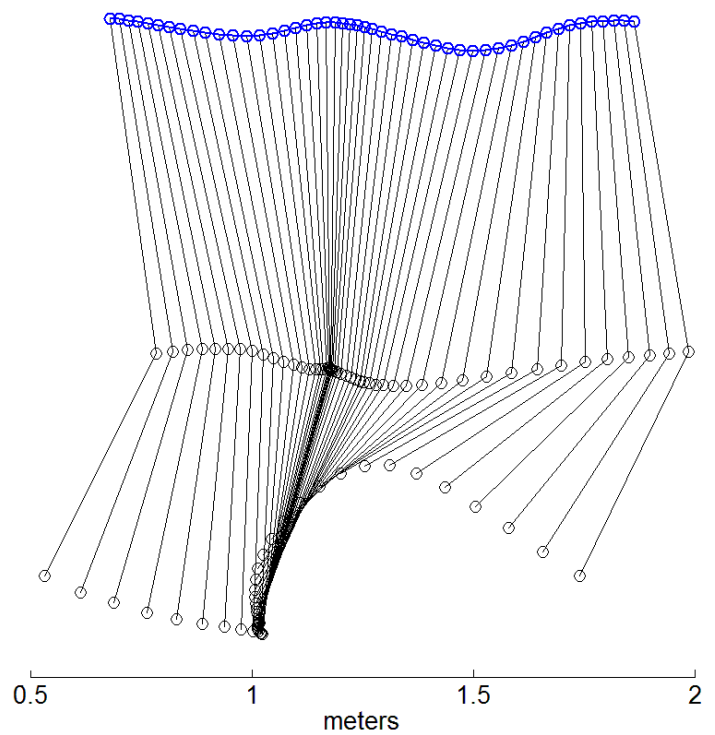

Figure 10: "Stroboscopic" image showing step of a leg in the $1 \mathrm{~g}$, in-water test case

The second stroboscopic image presents a gait cycle in the underwater, $1 g$ environment. Note that, compared with the dry environment, the vertical hip displacement, hip angle, and knee bend angle are more pronounced. These differences likely reflect the natural tendency of human subjects to try to minimize their drag profile when moving through water - raising the knee and foot higher during the forward swing have the effect of reducing the frontal area presented to the water by the leg as it moves forward. 


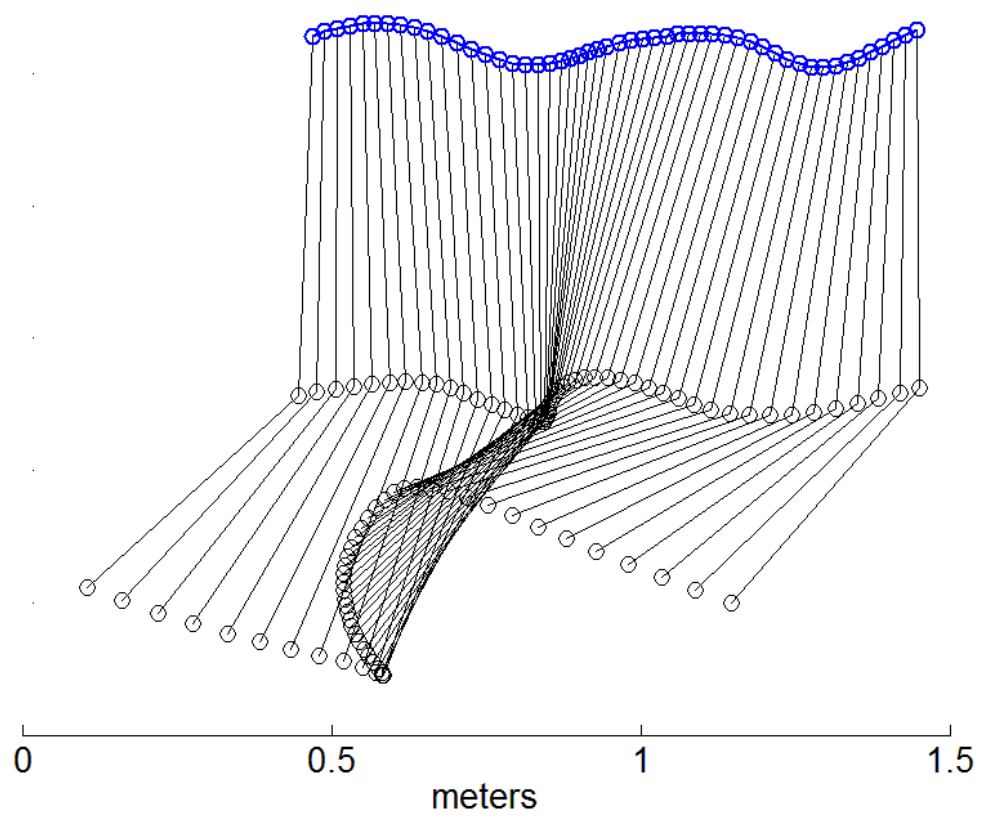

Figure 11: Stroboscopic image showing one step of a leg in the $1 / 6^{\text {th }} \mathrm{g}$, in-water test case

The final stroboscopic image shows a typical stride in the underwater, lunar gravityballasted environment. Knee bend in particular is even more exaggerated in this example than in the underwater $1 g$ environment. 


\section{Chapter 6: Motion-Capture Data Analysis}

\section{Equations for non-dimensionalized quantities}

In order to allow for meaningful comparisons between the different test subjects and gravitational environments, gait metrics were non-dimensionalized. By the dynamic similarity hypothesis, gaits for the same leg length, gravitational environment, and body mass should exhibit the same dynamic behavior. Thus, the basis for nondimensionalization used throughout this thesis is the subject leg length $l$, the subject body mass $\boldsymbol{M + 2 m}$, and the gravitational acceleration (actual or simulated with ballast), $g$. The following table lists equations for the non-dimensionalization of basic physical quantities used in the analyses below.

\begin{tabular}{|c|c|}
\hline Quantity & Equation \\
\hline Length & ND_length $=\frac{\text { length }}{l}$ \\
\hline Time & ND_time $=\frac{\text { time }}{\sqrt{l / g}}$ \\
\hline Speed & ND_speed $=\frac{\text { speed }}{\sqrt{l g}}$ \\
\hline Frequency & ND_frequency $=\frac{\text { frequency }}{\sqrt{g / l}}$ \\
\hline
\end{tabular}


In addition to these quantities, two more non-dimensional quantities are defined for use in the analyses below:

\begin{tabular}{|c|c|}
\hline Quantity & Equation \\
\hline Froude number & $F r=\frac{(\text { speed })^{2}}{l g}=(\text { ND_speed })^{2}$ \\
\hline Velocity exponent $\beta$ & $\beta=\log _{N D \_s p e e d} N D_{-} s t e p \_l e n g t h$ \\
& $=\frac{\log N D_{-} \text {step_length }}{\log N D_{-} \text {speed }}$ \\
\hline
\end{tabular}

The significance of the velocity exponent $\beta$ is its value in predicting the walk-run transition from the relationship of step length to gait speed. In dry, $1 \mathrm{~g}$ environments, walk-run transition occurs at a particular value of $\beta$, cited to be between 0.42 and 0.5 , regardless of subject anthropometry. ${ }^{[9][10]}$

\section{Identifying gait transition speed}

By observation of the video data recorded for each motion-capture trial, it is possible to subjectively categorize each gait as a walking or running gait. However, in order to generate a justifiable estimate of walk-run transition speed, it was necessary to create an objective measure of gait type based on the recorded data. 
Simplified model gaits may be strictly classified into walking, hybrid or transitional gaits, and "ideal" running gaits, with the latter characterized by instantaneous, impulsive ground contact. ${ }^{[17][8]}$ In real, physical running gaits, however, there is always some finite period of ground contact during the stride, which may or may not be ignored in modeling the gait. ${ }^{[17]}$ For the purpose of this analysis, "running gait" will refer to all such hybrid walk-run gaits, in which there is are finite periods of both single support and no support during each stride. The gait transition speed of interest, and the speed which is addressed below, is the speed at which the "no support" phase, in which both feet leave the ground, becomes non-negligible, rather than necessarily dominant.

In order to generate an estimate for the walk-run gait transition speed, a metric "isrun" was constructed to quantify the extent to which each recorded gait typified a walking or running gait. Such a metric would ideally be based directly on the physical definition of the gait transition, rather than rely on any prior expectation for the value of a dynamical gait metric, such as stride frequency or length, at the transition speed.

In order to achieve this goal, the "isrun" metric considered the height above the treadmill reached by the bottoms of the feet during each stride. In a purely walking gait, at least one foot is in contact with the tread surface at all times, and thus the height of the lower foot above the tread surface is expected to be zero at all times. Theoretically, any gait for which this is found to be true can be confidently identified 
as a walking gait, and any gait for which the lower foot achieves a positive height above the treadmill at some point in the stride may be identified as a running gait.

Practically, however, noise and measurement error in the experimental setup create a need for a more robust metric. The height of the lower foot occasionally shows a small positive value even in purely walking gaits as identified by observation. Therefore, a margin of error must be applied. A cutoff height of $3 \mathrm{~cm}$ (rather than 0 $\mathrm{cm}$ ) was found, through trial and error, to result in gait categorizations which closely matched subjective observation.

Secondly, because of variation over the course of each run, gait type was determined for each individual stride in a binary fashion $(0=$ indicative of a walking gait, $1=$ indicative of a running gait). These values were then averaged over the course of each trial, generating a value "meanisrun" which is the percentage of strides in a given trial which indicate a running gait. Because transitional gaits tend to involve less separation from the running surface than faster running gaits, "meanisrun" gives some indication of the extent to which a gait may be considered a walking or a running gait, though this is largely a qualitative indication.

The walk-run transition speed is expected to vary across subjects and environments due to different anthropometries and gravitational accelerations. ${ }^{[4][8]}$ Therefore, the non-dimensional Froude number is used to allow analysis incorporating runs from different subjects and environments. ${ }^{[4][13]}$ The Froude number, abbreviated Fr, is equal 
to the square of velocity divided by $g l$, gravitational acceleration multiplied by subject leg length. It is also identical to the square of the non-dimensional velocity, also used in this study. Use of the Froude number as the independent variable rests on the idea of dynamic similarity of gaits; that is, that gaits at different velocities and gravities will have similar characteristics at the same Froude number. The Froude number is equivalent to the ratio of inertial to gravitational forces acting on the object. ${ }^{[13][3]}$ Research by Alexander and Jayes suggests that the Froude number is a valuable means of assessing dynamic similarity of gaits, though there is some evidence that is its not ideal. ${ }^{[1][4][3]}$

\section{Gait transition speed analysis and results}

The following table includes Froude number and "meanisrun" data for all motioncapture trials:

\section{Running gait percentage and Froude number for each motion-capture trial}

\begin{tabular}{|c|c|c|c|c|c|c|c|}
\hline \multirow[b]{2}{*}{$\begin{array}{c}\text { Subject } \\
\#\end{array}$} & \multirow[b]{2}{*}{$\begin{array}{l}\text { Tread } \\
\text { speed }\end{array}$} & \multicolumn{2}{|c|}{ Dry $1 \mathrm{~g}$} & \multicolumn{2}{|c|}{ Wet $1 \mathrm{~g}$} & \multicolumn{2}{|c|}{ Wet lunar } \\
\hline & & Run \% & $\mathbf{F r}$ & Run \% & Fr & Run \% & $\mathbf{F r}$ \\
\hline 2 & low & $1.7 \%$ & 0.038 & $30.0 \%$ & 0.036 & $100.0 \%$ & 0.252 \\
\hline 3 & low & $0.0 \%$ & 0.037 & $0.0 \%$ & 0.033 & $100.0 \%$ & 0.261 \\
\hline 4 & low & $0.0 \%$ & 0.032 & $0.0 \%$ & 0.031 & $100.0 \%$ & 0.163 \\
\hline 5 & low & $0.0 \%$ & 0.041 & $0.0 \%$ & 0.038 & $100.0 \%$ & 0.303 \\
\hline 6 & low & $0.0 \%$ & 0.044 & $3.5 \%$ & 0.042 & $94.0 \%$ & 0.246 \\
\hline 2 & medium & $0.0 \%$ & 0.082 & $34.7 \%$ & 0.076 & $100.0 \%$ & 0.536 \\
\hline 3 & medium & $0.0 \%$ & 0.081 & $49.2 \%$ & 0.064 & $100.0 \%$ & 0.535 \\
\hline 4 & medium & $0.0 \%$ & 0.075 & $95.9 \%$ & 0.061 & $100.0 \%$ & 0.376 \\
\hline 5 & medium & $0.0 \%$ & 0.087 & $19.7 \%$ & 0.077 & $100.0 \%$ & 0.561 \\
\hline 6 & medium & $0.0 \%$ & 0.095 & $46.9 \%$ & 0.091 & $96.4 \%$ & 0.530 \\
\hline 2 & high & $0.0 \%$ & 0.142 & $66.0 \%$ & 0.126 & $100.0 \%$ & 0.772 \\
\hline 3 & high & $0.0 \%$ & 0.138 & $100.0 \%$ & 0.117 & $100.0 \%$ & 1.042 \\
\hline
\end{tabular}




\begin{tabular}{|ll|ll|ll|ll|}
4 & high & $0.0 \%$ & 0.127 & $96.0 \%$ & 0.095 & $100.0 \%$ & 0.688 \\
5 & high & $0.0 \%$ & 0.150 & $75.0 \%$ & 0.148 & $100.0 \%$ & 0.961 \\
6 & high & $0.0 \%$ & 0.160 & $92.0 \%$ & 0.084 & $100.0 \%$ & 0.914 \\
\hline
\end{tabular}

The following plot shows the relationship between Froude number (plotted logarithmically) and the percentage of strides in a given trial indicating a running gait. Each individual trial is marked by an " $\mathrm{x}$ ".

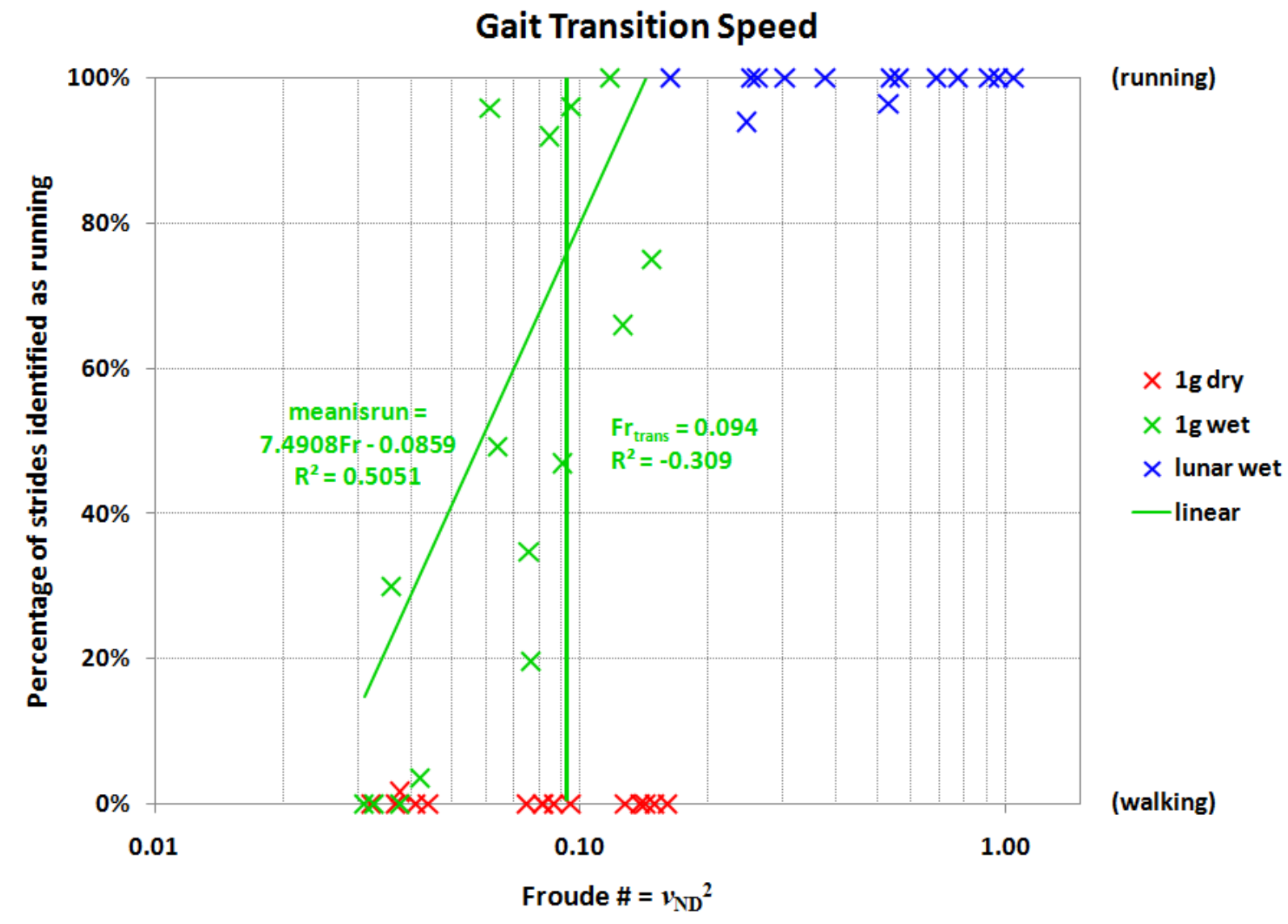

Figure 12: Gait Transition Speed

Observing the table above, the three test environments combined show the expected trend from walking gaits at lower Froude numbers to running gaits at higher Froude numbers. ${ }^{[8]}$ Looking at each environment in turn, it is apparent that all of the dry, $1 \mathrm{~g}$ 
trials show a walking gait. It would be necessary to extend the collected data to higher tread speeds in order to see the beginning of a walk-run transition in the dry $1 g$ environment.

In contrast, the underwater, lunar gravity trials overwhelmingly indicate running gaits. It would be necessary to extend the collected data to lower tread speeds in order to find walking gaits in this environment.

The data for the underwater, $1 \mathrm{~g}$ environment, finally, show a spectrum of gait types, ranging from walking at lower Froude numbers to running at higher Froude numbers. From these data, two approaches are taken to address the walk-run transition. The first approach is a linear regression fit to the data for the underwater, $1 g$ environment, producing an expression for the expected percentage of strides identified as running, as a function of Froude number: meanisrun $=7.491 \mathrm{Fr}-0.0859 . \quad$ Taking $\quad$ meanisrun $=0 \% \quad$ and meanisrun $=100 \%$, the linear relationship indicates that the walk-run transition occurs over a range of Froude numbers, between $F r=0.0115$ and $F r=0.145$.

The second approach assumes that gait type undergoes a step transition from walking to running at some particular Froude number. By switching the $x$ - and $y$-coordinates and taking a least-squares regression of the underwater lunar gravity data while constraining the slope to be zero, a value of $F r=0.094$ is found. This indicates that 
all gaits at Froude numbers lower than 0.094 should be expected to be walking gaits, and all gaits at higher Froude numbers should be expected to be running gaits.

Note that, for either approach, there is some discrepancy between the data from the underwater environments and the dry, $1 g$ data with regard to the walk-run transition speed. In each case, the dry, $1 g$ data indicate walking gaits at Froude numbers higher than those at which the underwater, $1 \mathrm{~g}$ data indicate a transition to a running gait. This discrepancy hints at an effect of the underwater environment on gait transition speed, presumably due to drag and/or virtual mass effects. The indication is that, even when gravitational acceleration is corrected for with ballast, walk-run transition occurs at lower speeds in the underwater environment. However, without an extended dry $1 g$ data set including higher tread speeds, it is not possible to quantify the magnitude of this effect.

Unfortunately, hardware limitations and safety considerations precluded the collection of data at a broader range of tread speeds for this study. At lower tread speeds, friction and low inertia in the treadmill resulted in "jerking", rather than smooth motion, of the tread underneath a moving subject. Much higher speeds resulted in over-current faults in the treadmill power supply, and risked overly exerting subjects, leading to the potential for injury. 


\section{Gait comparison across test environments}

Five dynamical gait metrics - stride frequency, step length, maximum hip angle, vertical displacement of the torso, and the non-dimensional velocity exponent $\beta-$ were used to compare gaits between the three physical test environments. ${ }^{[10]}$ Comparisons were drawn between dry land and underwater environments at $1 \mathrm{~g}$, and between $1 g$-ballasted and lunar gravity-ballasted underwater environments. For each of these gait metrics, an analysis of covariance was performed, using the Analysis of Covariance Tool ("aoctool") and "multcompare" functions in the Matlab Statistics Toolbox.

Because of the variation in measured gait speed across the three environments and between subjects, a standard analysis of variance (ANOVA) could not reliably be used to assess the influence of environment on variance of means between sample groups for the three environments. Analysis of covariance (ANCOVA) identifies the portion of variance between sample groups that is not accounted for by one or more continuous variables. In this case, each gait metric is a function of one continuous variable (gait speed) and one discreet "dummy" variable (environment). It is the variance caused by the latter variable that is of primary interest.

Note that the analysis of covariance was conducted on non-dimensionalized data, in order to eliminate variance caused by variation in subject anthropometry. The basis for non-dimensionalization consisted of the subject's leg length, the subject's total body mass, and the simulated gravitational acceleration of each 
environment. ${ }^{[9][17][10][12]}$ Both gait metric values and gait speed were nondimensionalized.

The analysis of covariance produced a linear regression fit for each metric in each of the three environments, as a function of gait speed. In addition, a 95\% confidence band was generated for each regression line. This confidence band defines a twodimensional region for which there is a $95 \%$ chance that the regression line for the population will lie within the region. This should not be confused with a prediction band, a wider region with a $95 \%$ probability of encompassing one additional observation. The sample data points, linear regression lines, and 95\% confidence bands for each gait metric were plotted together. These plots are presented and discussed in detail below.

A key goal of performing the analysis of covariance was to test for the significance of differences in the regression coefficients between the three environments. In order to perform this hypothesis testing, the "stats" output structure from aoctool was fed into the "multcompare" function, which provided the results of multiple comparison testing in order to identify significant differences in the linear fit coefficients at the 95\% threshold. The "multcompare" function uses the Tukey-Kramer "honestly significant diffence" method, which is based on a Studentized range distribution.

The Matlab code written to perform these analyses of the motion-capture data can be found in Appendices 5i and 5j. 


\section{Gait comparison analysis}

The following table presents the coefficient values (slope and intercept) for each metric in each of the three environments, as well as the two comparisons of interest the comparison between dry and underwater environments at $1 \mathrm{~g}$, and the comparison between $1 \mathrm{~g}$ and lunar gravities in the underwater environment. Each comparison provides a range of possible values, within a $95 \%$ threshold, for the difference between the coefficient values in the two environments being compared. If this range does not include zero, the null hypothesis is rejected, indicating a significant difference between the coefficients at the $95 \%$ confidence level.

\section{Linear Regression Coefficients and ANCOVA Hypothesis Testing}

\begin{tabular}{|c|c|c|c|c|c|c|c|c|c|c|c|c|c|c|}
\hline Metric & Parameter & \multicolumn{2}{|c|}{ dry 1g } & \multicolumn{2}{|c|}{ wet $1 g$} & \multicolumn{2}{|c|}{ wet lunar } & \multicolumn{2}{|c|}{$\frac{\text { dry vs. wet }}{(95 \%)}$} & \multirow{2}{*}{\begin{tabular}{c|} 
Reject \\
$\underline{\text { Ho? }}$ \\
-
\end{tabular}} & \multicolumn{3}{|c|}{$\frac{1 \mathrm{~g} \text { vs. lunar }}{(95 \%)}$} & \multirow{2}{*}{$\frac{\underline{\text { Reject }}}{\underline{\text { Ho? }}}$} \\
\hline \multirow{2}{*}{$\begin{array}{l}\text { swing freq. } \\
(\omega)\end{array}$} & slope & $0.41 \pm$ & \pm 0.06 & $0.36=$ & \pm 0.07 & $0.08 \pm$ & 0.03 & $0.05 \pm$ & 0.24 & & 0.28 & \pm & 0.19 & \\
\hline & intercept & $0.12 \pm$ & \pm 0.02 & $0.09=$ & \pm 0.02 & $0.09 \pm$ & 0.02 & $0.03 \pm$ & 0.07 & - & 0.00 & \pm & 0.07 & - \\
\hline \multirow{2}{*}{ step length } & slope & $1.09 \pm$ & \pm 0.42 & $1.28=$ & \pm 0.49 & $0.93 \pm$ & 0.17 & $-0.19 \pm$ & 1.56 & - & 0.35 & \pm & 1.26 & - \\
\hline & intercept & $0.29 \pm$ & \pm 0.12 & $0.37=$ & \pm 0.13 & $0.36 \pm$ & 0.12 & $-0.08 \pm$ & 0.44 & - & 0.01 & \pm & 0.44 & - \\
\hline \multirow{2}{*}{$\begin{array}{l}\text { max. hip } \\
\text { angle }(\phi)\end{array}$} & slope & $0.94 \pm$ & \pm 0.36 & $1.30=$ & \pm 0.42 & $0.17 \pm$ & 0.14 & $-0.36 \pm$ & 1.34 & - & 1.13 & \pm & 1.08 & sig diff \\
\hline & intercept & $0.34 \pm$ & \pm 0.11 & $0.33=$ & \pm 0.11 & $0.41 \pm$ & 0.11 & $0.01 \pm$ & 0.38 & - & -0.08 & \pm & 0.38 & - \\
\hline \multirow{2}{*}{$\begin{array}{l}\text { torso vertical } \\
\text { displacement }\end{array}$} & slope & $0.10 \pm$ & \pm 0.07 & $0.14=$ & \pm 0.08 & $-0.03 \pm$ & 0.03 & $-0.04 \pm$ & 0.27 & - & 0.17 & \pm & 0.22 & - \\
\hline & intercept & $0.00 \pm$ & \pm 0.02 & $0.01=$ & \pm 0.02 & $0.10 \pm$ & 0.02 & $0.00 \pm$ & 0.08 & - & -0.09 & \pm & 0.08 & sig diff \\
\hline \multirow{2}{*}{$\begin{array}{c}\text { velocity } \\
\text { exponent }(\beta)\end{array}$} & slope & $-0.31 \pm$ & \pm 0.35 & $-0.67=$ & \pm 0.42 & $-0.76 \pm$ & 0.14 & $0.36 \pm$ & 1.33 & - & 0.09 & \pm & 1.07 & - \\
\hline & intercept & $0.49 \pm$ & \pm 0.11 & $0.43=$ & \pm 0.11 & $0.51 \pm$ & 0.10 & $0.06 \pm$ & 0.38 & - & -0.09 & \pm & 0.38 & - \\
\hline
\end{tabular}

In order to discuss the various regression fits and comparisons in a physically meaningful way, the linear fit data above were re-dimensionalized on the basis of a $50^{\text {th }}$ percentile adult anthropometry (for the general population, not the test subject sample group). ${ }^{[1]]}$ These re-dimensionalized data are presented below. In addition to the two regression coefficients (slope and intercept), metric values and comparisons are presented for a gait speed of $1.5 \mathrm{~m} / \mathrm{s}$, which is approximately the average redimensionalized gait speed. Note that the range provided for the two comparison 
colums below is a standard error, rather than the $95 \%$ threshold used for hypothesis rejection above. These error values are equal to the $l^{2}$ norm of the error values of the coefficients being compared.

\section{Re-dimensionalized Regression Coefficients and Comparisons}

\begin{tabular}{|c|c|c|c|c|c|c|c|c|c|c|c|c|c|c|c|c|}
\hline \multirow[b]{2}{*}{ Metric } & \multirow[b]{2}{*}{ Parameter } & & & & \multicolumn{2}{|c|}{ Environment } & & & & & & & & & & \\
\hline & & \multicolumn{3}{|c|}{ dry $1 g$} & \multicolumn{2}{|c|}{ wet $1 g$} & \multicolumn{3}{|c|}{ wet lunar } & \multicolumn{3}{|c|}{ dry vs. wet } & \multicolumn{3}{|c|}{$\underline{1 g}$ vs. lunar } & Units \\
\hline \multirow{3}{*}{$\begin{array}{l}\text { swing } \\
\text { freq. }(\omega)\end{array}$} & slope & 0.47 & \pm & 0.07 & $0.42 \pm$ & \pm 0.09 & 0.09 & \pm & 0.03 & 0.06 & \pm & 0.11 & 0.33 & \pm 0 & 0.09 & $\mathrm{~Hz} /(\mathrm{m} / \mathrm{s})$ \\
\hline & intercept & 0.40 & \pm & 0.06 & $0.29 \pm$ & \pm 0.07 & 0.12 & \pm & 0.03 & 0.11 & \pm & 0.09 & 0.17 & \pm 0 & 0.07 & $\mathrm{~Hz}$ \\
\hline & $@ 1.5 \mathrm{~m} / \mathrm{s}$ & 1.11 & \pm & 0.13 & $0.92 \pm$ & \pm 0.15 & 0.26 & \pm & 0.05 & 0.19 & \pm & 0.19 & 0.66 & \pm 0 & 0.16 & $\mathrm{~Hz}$ \\
\hline \multirow{3}{*}{$\begin{array}{c}\text { step } \\
\text { length }\end{array}$} & slope & 0.32 & \pm & 0.12 & $0.38 \pm$ & \pm 0.15 & 0.68 & \pm & 0.12 & -0.06 & \pm & 0.19 & -0.30 & \pm 0 & 0.19 & $\mathrm{~m} /(\mathrm{m} / \mathrm{s})$ \\
\hline & intercept & 0.25 & \pm & 0.11 & $0.32 \pm$ & \pm 0.12 & 0.31 & \pm & 0.11 & -0.07 & \pm & 0.16 & 0.01 & \pm 0 & 0.16 & $\mathrm{~m}$ \\
\hline & $@ 1.5 \mathrm{~m} / \mathrm{s}$ & 0.73 & \pm & 0.21 & $0.89 \pm$ & \pm 0.25 & 1.33 & \pm & 0.21 & -0.16 & \pm & 0.33 & -0.44 & \pm 0 & 0.32 & $\mathrm{~m}$ \\
\hline \multirow{3}{*}{$\begin{array}{l}\text { max. hip } \\
\text { angle }(\phi)\end{array}$} & slope & 0.32 & \pm & 0.12 & $0.45 \pm$ & \pm 0.14 & 0.15 & \pm & 0.12 & -0.12 & \pm & 0.19 & 0.30 & \pm 0 & 0.19 & $\mathrm{rad} /(\mathrm{m} / \mathrm{s})$ \\
\hline & intercept & 0.34 & \pm & 0.11 & $0.33 \pm$ & \pm 0.11 & 0.41 & \pm & 0.11 & 0.01 & \pm & 0.16 & -0.08 & \pm 0 & 0.16 & rads \\
\hline & $@ 1.5 \mathrm{~m} / \mathrm{s}$ & 0.82 & \pm & 0.21 & $1.00 \pm$ & \pm 0.24 & 0.63 & \pm & 0.21 & -0.18 & \pm & 0.32 & 0.37 & \pm 0 & 0.32 & rads \\
\hline \multirow{3}{*}{$\begin{array}{c}\text { torso } \\
\text { vertical } \\
\text { disp. }\end{array}$} & slope & 0.03 & \pm & 0.02 & $0.04 \pm$ & \pm 0.02 & -0.02 & \pm & 0.02 & -0.01 & \pm & 0.03 & 0.06 & \pm 0 & 0.03 & $\mathrm{~m} /(\mathrm{m} / \mathrm{s})$ \\
\hline & intercept & 0.00 & \pm & 0.02 & $0.01 \pm$ & \pm 0.02 & 0.08 & \pm & 0.02 & 0.00 & \pm & 0.03 & -0.08 & \pm 0 & 0.03 & $\mathrm{~m}$ \\
\hline & $@ 1.5 \mathrm{~m} / \mathrm{s}$ & 0.05 & \pm & 0.04 & $0.07 \pm$ & \pm 0.04 & 0.05 & \pm & 0.04 & -0.02 & \pm & 0.06 & 0.02 & \pm 0 & 0.06 & $\mathrm{~m}$ \\
\hline \multirow{3}{*}{$\begin{array}{l}\text { velocity } \\
\text { exponent } \\
(\beta)\end{array}$} & slope & -0.11 & \pm & 0.12 & $-0.23 \pm$ & \pm 0.14 & -0.64 & \pm & 0.12 & 0.12 & \pm & 0.19 & 0.41 & \pm 0 & 0.19 & $1 /(\mathrm{m} / \mathrm{s})$ \\
\hline & intercept & 0.49 & \pm & 0.11 & $0.43 \pm$ & \pm 0.11 & 0.51 & \pm & 0.10 & 0.06 & \pm & 0.15 & -0.09 & \pm 0 & 0.15 & - \\
\hline & $@ 1.5 \mathrm{~m} / \mathrm{s}$ & 0.33 & \pm & 0.21 & $0.08 \pm$ & \pm 0.24 & -0.44 & \pm & 0.21 & 0.25 & \pm & 0.32 & 0.52 & \pm 0 & 0.32 & - \\
\hline
\end{tabular}

The rows of the above tables are reproduced, with the corresponding plots, below. 
Linear regression and confidence band plots

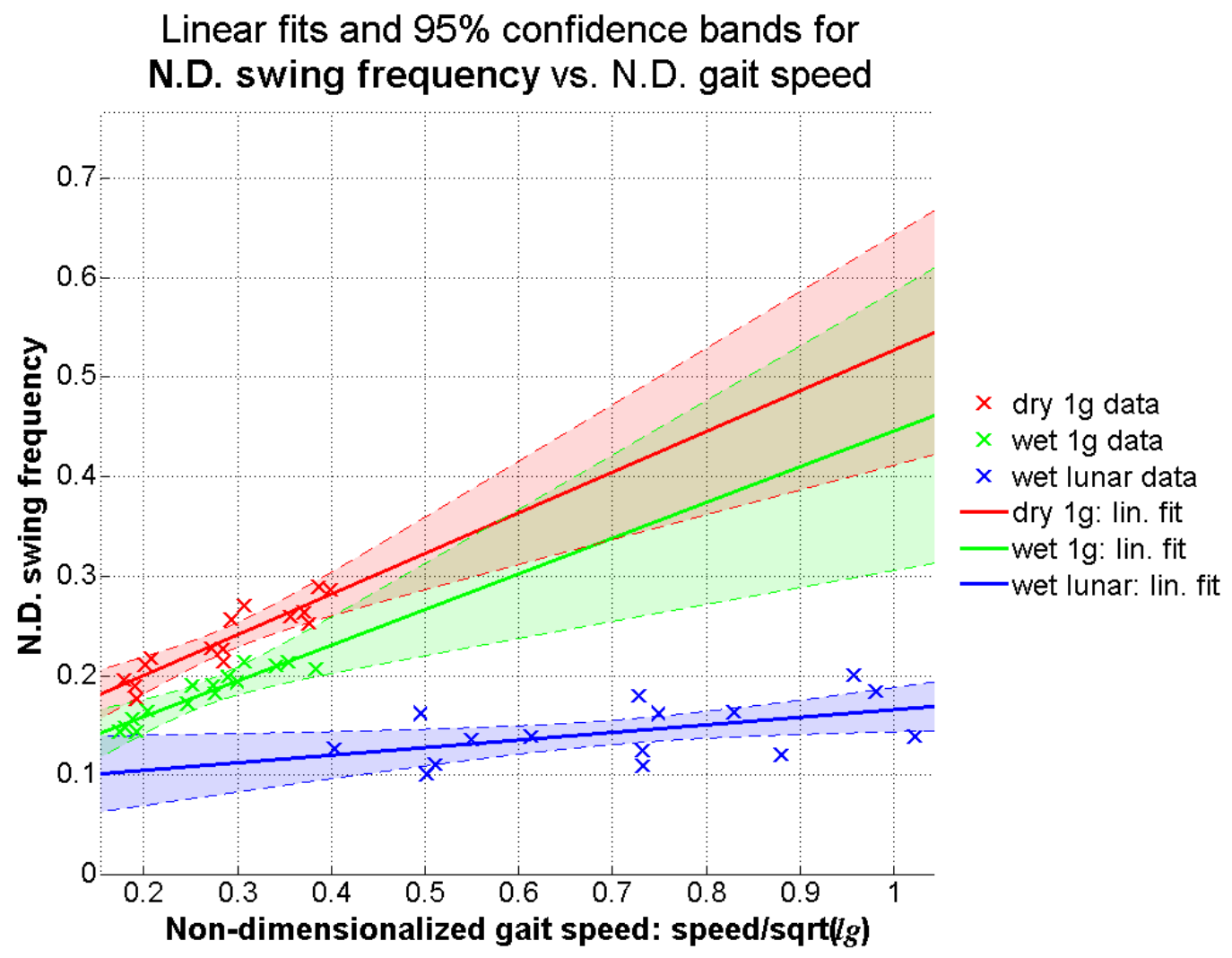

Figure 13: Stride frequency vs. gait speed

\begin{tabular}{|c|c|c|c|c|c|c|c|c|c|c|}
\hline$\underline{\text { Metric }}$ & Parameter & dry $1 g$ & wet $1 \mathrm{~g}$ & wet lunar & $\frac{\text { dry vs. wet }}{(95 \%)}$ & $\begin{array}{l}\text { Reject } \\
\text { H0? }\end{array}$ & \multicolumn{3}{|c|}{$\frac{1 \mathrm{~g} \text { vs. lunar }}{(95 \%)}$} & $\frac{\text { Reject }}{\underline{\text { H0? }}}$ \\
\hline \multirow{2}{*}{ 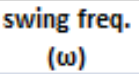 } & slope & $0.41 \pm 0.06$ & $0.36 \pm 0.07$ & $0.08 \pm 0.03$ & $0.05 \pm 0.24$ & - & 0.28 & \pm & 0.19 & sig diff \\
\hline & intercept & $0.12 \pm 0.02$ & $0.09 \pm 0.02$ & $0.09 \pm 0.02$ & $0.03 \pm 0.07$ & - & 0.00 & \pm & 0.07 & - \\
\hline
\end{tabular}

\begin{tabular}{|c|r|r|c|c|c|c|c|c|c|}
\hline metric & parameter & \multicolumn{1}{|c|}{ dry 1g } & wet 1g & wet lunar & \multicolumn{1}{c|}{ dry vs. wet } & 1g vs. lunar & units \\
\hline \multirow{2}{*}{$\begin{array}{c}\text { swing } \\
\text { freq. }(\omega)\end{array}$} & slope & $0.47 \pm 0.07$ & $0.42 \pm 0.09$ & $0.09 \pm 0.03$ & $0.06 \pm 0.11$ & $0.33 \pm 0.09$ & $\mathrm{~Hz} /(\mathrm{m} / \mathrm{s})$ \\
& intercept & $0.40 \pm 0.06$ & $0.29 \pm 0.07$ & $0.12 \pm 0.03$ & $0.11 \pm 0.09$ & $0.17 \pm 0.07$ & $\mathrm{~Hz}$ \\
& $@ 1.5 \mathrm{~m} / \mathrm{s}$ & $1.11 \pm \mathbf{0 . 1 3}$ & $\mathbf{0 . 9 2} \pm \mathbf{0 . 1 5}$ & $\mathbf{0 . 2 6} \pm \mathbf{0 . 0 5}$ & $\mathbf{0 . 1 9} \pm \mathbf{0 . 1 9}$ & $\mathbf{0 . 6 6} \pm \mathbf{0 . 1 6}$ & $\mathrm{Hz}$ \\
\hline
\end{tabular}


This plot presents the linear regressions and confidence bands for nondimensionalized stride frequency as a function of non-dimensionalized gait speed, in each of the three environments: dry land at $1 g$, underwater at $1 g$, and underwater at lunar gravity. Note that, because of the use of gravitational acceleration as a basis for non-dimensionalization, the data points collected in the lunar gravity environment have significantly higher non-dimensionalized gait speeds than the data points collected in the $1 \mathrm{~g}$ environments, although the true speeds are in fact comparable between all three environments.

These three fits show a trend of increasing step frequency with gait speed, as would be expected. ${ }^{[9]}$ The data indicate a significant difference in slope between the $1 \mathrm{~g}$ and lunar gravity underwater environments. Observation of the plot and data table show a clear separation between the lunar gravity environment and the two $1 \mathrm{~g}$ environments, while the two one $g$ environments produce similar values. The $1 g$ fits show a steeper positive slope, producing higher stride frequencies in $1 \mathrm{~g}$ than in lunar gravity. This corresponds with expectations. ${ }^{[14]}$

At a typical $1 \mathrm{~g}$ gait speed of $1.5 \mathrm{~m} / \mathrm{s}$ for a $50^{\text {th }}$ percentile adult, the difference in stride frequency between dry and wet environments is small, at $0.19 \pm 0.19 \mathrm{~Hz}$. The difference is much greater between $1 g$ and lunar environments, with $1 g$ gaits $0.66 \pm 0.16 \mathrm{~Hz}$ faster than lunar gaits. 


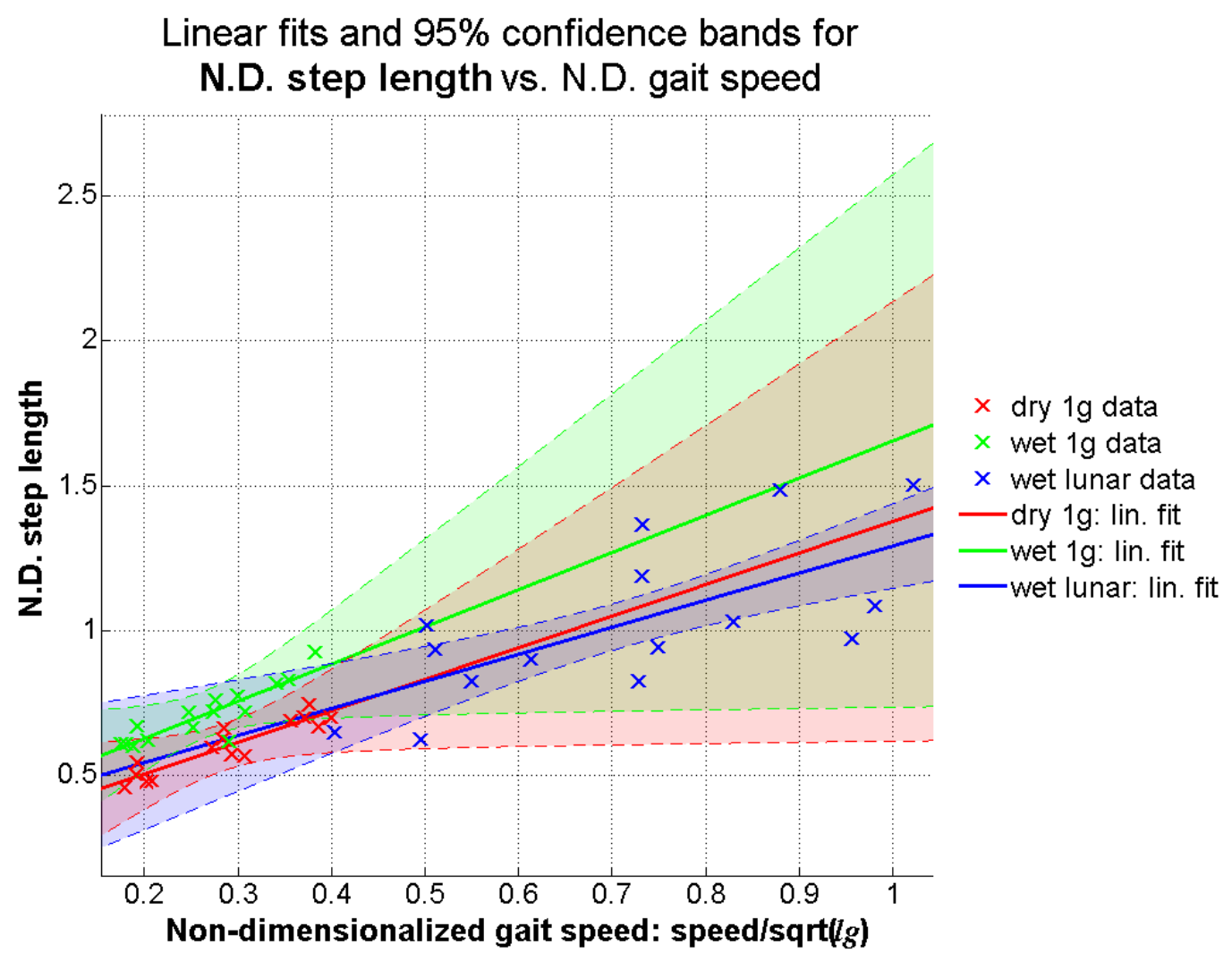

Figure 14: Step length vs. gait speed

\begin{tabular}{|c|c|c|c|c|c|c|c|c|c|c|}
\hline$\underline{\text { Metric }}$ & Parameter & $\underline{\text { dry } 1 g}$ & wet $1 g$ & wet lunar & $\frac{\text { dry vs. wet }}{(95 \%)}$ & $\begin{array}{l}\text { Reject } \\
\text { H0? }\end{array}$ & $\frac{1 g}{1}$ & $95 \%$ & & $\frac{\text { Reject }}{\text { H0? }}$ \\
\hline \multirow{2}{*}{ step length } & slope & $1.09 \pm 0.42$ & $1.28 \pm 0.49$ & $0.93 \pm 0.17$ & $-0.19 \pm 1.56$ & - & 0.35 & \pm & 1.26 & - \\
\hline & intercept & $0.29 \pm 0.12$ & $0.37 \pm 0.13$ & $0.36 \pm 0.12$ & $-0.08 \pm 0.44$ & - & 0.01 & \pm & 0.44 & - \\
\hline
\end{tabular}

\begin{tabular}{|c|c|c|c|c|c|c|c|c|c|}
\hline metric & parameter & dry 1g & wet $1 g$ & wet lunar & $\underline{\mathrm{dry}}$ & vs. & & 1g vs. lunar & units \\
\hline \multirow{3}{*}{$\begin{array}{c}\text { step } \\
\text { length }\end{array}$} & slope & $0.32 \pm 0.12$ & $0.38 \pm 0.15$ & $0.68 \pm 0.12$ & -0.06 & \pm & 0.19 & $-0.30 \pm 0.19$ & $\mathrm{~m} /(\mathrm{m} / \mathrm{s})$ \\
\hline & intercept & $0.25 \pm 0.11$ & $0.32 \pm 0.12$ & $0.31 \pm 0.11$ & -0.07 & \pm & 0.16 & $0.01 \pm 0.16$ & $\mathrm{~m}$ \\
\hline & $@ 1.5 \mathrm{~m} / \mathrm{s}$ & $0.73 \pm 0.21$ & $0.89 \pm 0.25$ & $1.33 \pm 0.21$ & -0.16 & \pm & 0.33 & $-0.44 \pm 0.32$ & $\mathrm{~m}$ \\
\hline
\end{tabular}

These three fits demonstrate a trend of increasing step length with gait speed, conforming to expectations. ${ }^{[10]}$ The null hypothesis is not rejected in any of the comparisons, and the plot and data table show a fairly close match between the three environments, with significant overlap of the confidence bands. 
At $1.5 \mathrm{~m} / \mathrm{s}$, the difference in step length between dry and wet $1 g$ environments is small and within the margin of error, at $-0.16 \pm 0.33 \mathrm{~m}$. The difference in step length between $1 \mathrm{~g}$ and lunar gravity underwater environments is somewhat larger, with lunar gravity producing steps which are $0.44 \pm 0.32 \mathrm{~m}$ longer than in $1 \mathrm{~g}$. This corresponds well with the expectation of a running gait in lunar gravity ${ }^{[3]}$, in which both feet leave the ground, and the subject is able to cover a significant "glide" distance during each step without a corresponding increase in the angle of the legs. [14][13] 


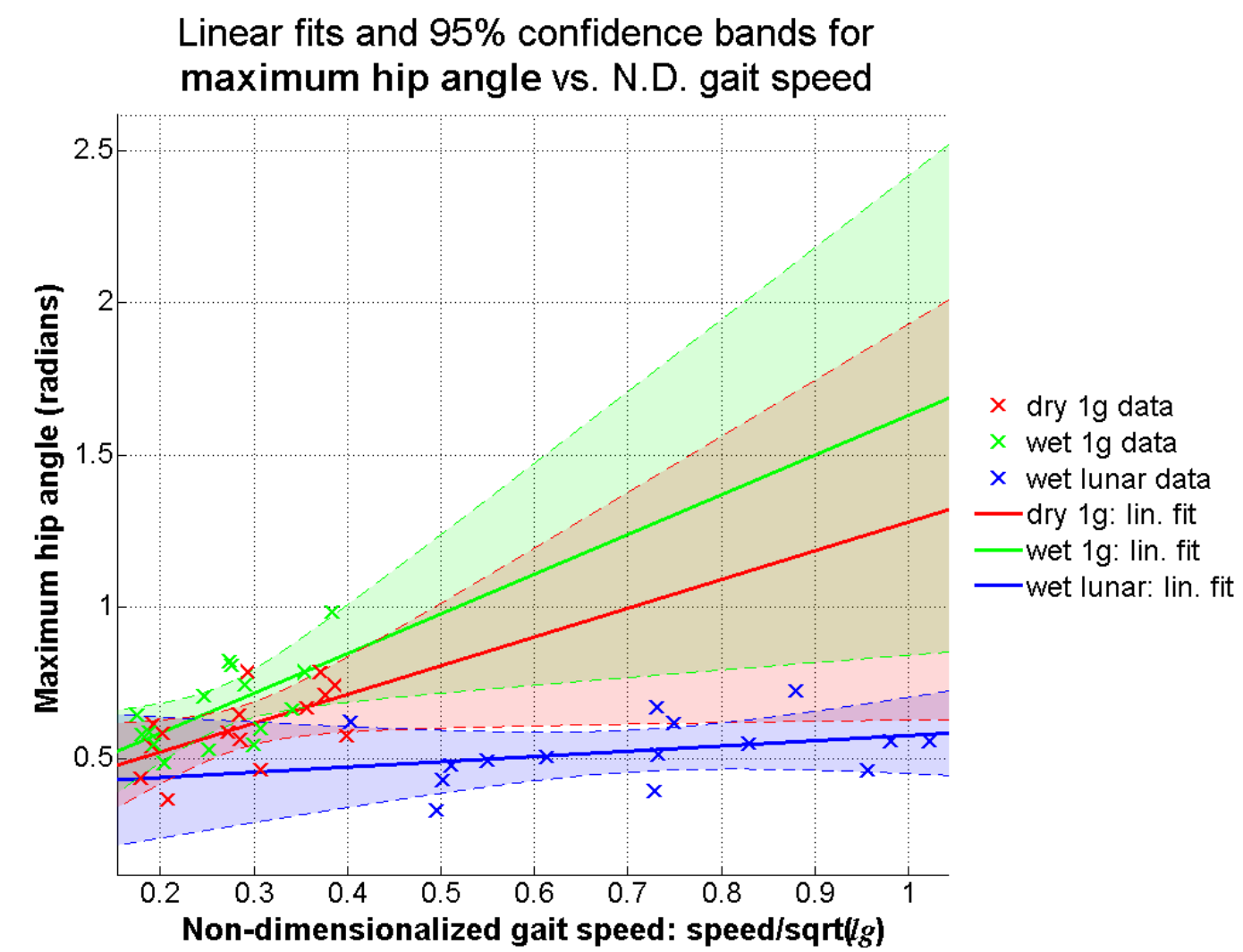

Figure 15: Maximum hip angle vs. gait speed

\begin{tabular}{|c|c|c|c|c|c|c|c|c|c|c|}
\hline Metric & Parameter & dry 1g & wet $1 \mathrm{~g}$ & wet lunar & $\frac{\text { dry vs. wet }}{(95 \%)}$ & $\begin{array}{l}\text { Reject } \\
\text { H0? }\end{array}$ & \multicolumn{3}{|c|}{$\frac{\text { 1g vs. Iunar }}{(95 \%)}$} & $\frac{\text { Reject }}{\text { H0? }}$ \\
\hline max. hip & slope & $0.94 \pm 0.36$ & $1.30 \pm 0.42$ & $0.17 \pm 0.14$ & $-0.36 \pm 1.34$ & - & 1.13 & \pm & 1.08 & sig diff \\
\hline angle $(\phi)$ & intercept & $0.34 \pm 0.11$ & $0.33 \pm 0.11$ & $0.41 \pm 0.11$ & $0.01 \pm 0.38$ & - & -0.08 & \pm & 0.38 & - \\
\hline
\end{tabular}

\begin{tabular}{|c|c|c|c|c|c|c|c|c|c|}
\hline metric & parameter & dry $1 g$ & wet $1 \mathrm{~g}$ & wet lunar & dry & v5. 1 & & 1g vs. lunar & units \\
\hline \multirow{3}{*}{$\begin{array}{r}\text { max. hip } \\
\text { angle }(\phi)\end{array}$} & slope & $0.32 \pm 0.12$ & $0.45 \pm 0.14$ & $0.15 \pm 0.12$ & -0.12 & \pm & 0.19 & $0.30 \pm 0.19$ & $\mathrm{rads} /(\mathrm{m} / \mathrm{s})$ \\
\hline & intercept & $0.34 \pm 0.11$ & $0.33 \pm 0.11$ & $0.41 \pm 0.11$ & 0.01 & \pm & 0.16 & $-0.08 \pm 0.16$ & rads \\
\hline & $@ 1.5 \mathrm{~m} / \mathrm{s}$ & $0.82 \pm 0.21$ & $1.00 \pm 0.24$ & $0.63 \pm 0.21$ & -0.18 & \pm & 0.32 & $0.37 \pm 0.32$ & rads \\
\hline
\end{tabular}

These three plots indicate a trend of increasing hip angle with gait speed. This matches well with the finding of increased step length with gait speed discussed above. However, the data indicate a significant difference in slope between $1 g$ and lunar gravity underwater environments. The lunar gravity fit has a much shallower 
slope than the $1 g$ fits, producing significantly smaller hip angles at higher gait speeds.

This is in line with expections; as indicated in the previous two plots, faster gait speed is achieved by a combination of longer and faster strides, regardless of the gait type employed. For walking gaits, as are expected in $1 \mathrm{~g}$, a longer stride with a fixed leg length requires a greater maximum hip angle during each step, simply by the geometry of the gait (the fixed-length legs form a triangle with the portion of walking surface covered during the step, with the hip angle opposite the walking surface). This constraint does not apply, however, to running gaits, in which forward distance is gained in a series of ballistic flights, whose distance does not depend on the angle between the legs. ${ }^{[10][17][13]}$

At $1.5 \mathrm{~m} / \mathrm{s}$, the difference in hip angle between dry and wet $1 \mathrm{~g}$ environments is small and within the margin of error, at $-0.18 \pm 0.32$ radians. The difference between $1 \mathrm{~g}$ and lunar gravity underwater environments is larger, with a hip angle $0.37 \pm 0.32$ radians greater in $1 \mathrm{~g}$ than in the lunar environment. 


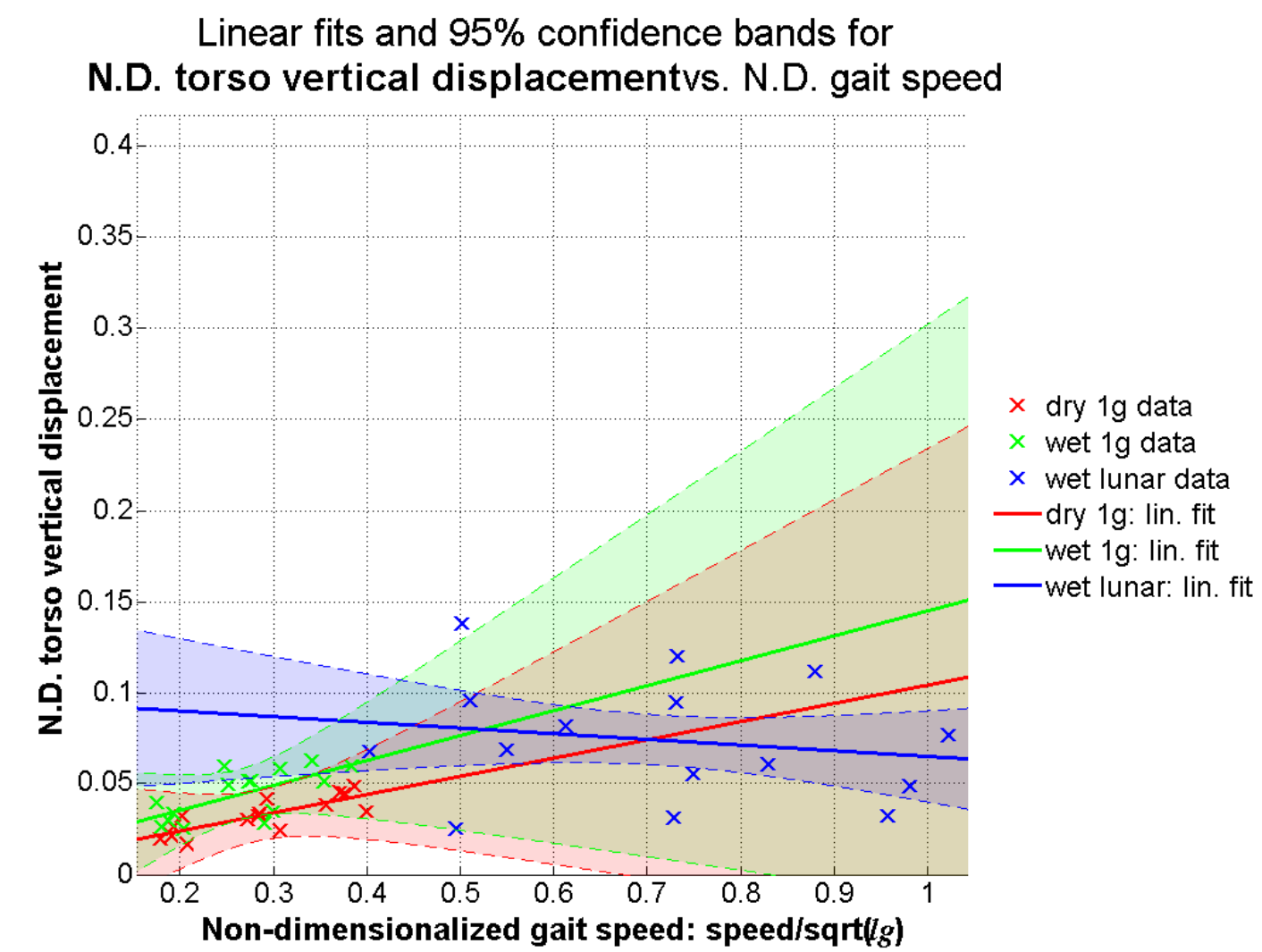

Figure 16: Vertical torso displacement vs. gait speed

\begin{tabular}{|c|c|c|c|c|c|c|c|c|c|c|}
\hline Metric & Parameter & dry 1g & wet $1 \mathrm{~g}$ & wet lunar & $\frac{\text { dry vs. wet }}{(95 \%)}$ & $\begin{array}{c}\text { Reject } \\
\text { H0? } \\
\end{array}$ & \multicolumn{3}{|c|}{$\frac{\text { 1g vs. lunar }}{(95 \%)}$} & $\frac{\text { Reject }}{\text { H0? }}$ \\
\hline & slope & $0.10 \pm 0.07$ & $0.14 \pm 0.08$ & $-0.03 \pm 0.03$ & $-0.04 \pm 0.27$ & - & 0.17 & \pm & 0.22 & - \\
\hline displacement & intercept & $0.00 \pm 0.02$ & $0.01 \pm 0.02$ & $0.10 \pm 0.02$ & $0.00 \pm 0.08$ & - & -0.09 & \pm & 0.08 & sig diff \\
\hline
\end{tabular}

\begin{tabular}{|c|c|c|c|c|c|c|c|c|c|}
\hline metric & parameter & dry $1 g$ & wet $1 \mathrm{~g}$ & wet lunar & \multicolumn{3}{|c|}{ dry vs. wet } & 1g vs. lunar & \multirow{2}{*}{$\frac{\text { units }}{\mathrm{m} /(\mathrm{m} / \mathrm{s})}$} \\
\hline \multirow{3}{*}{$\begin{array}{l}\text { torso } \\
\text { vertical } \\
\text { disp. }\end{array}$} & slope & $0.03 \pm 0.02$ & $0.04 \pm 0.02$ & $-0.02 \pm 0.02$ & -0.01 & \pm & 0.03 & $0.06 \pm 0.03$ & \\
\hline & intercept & $0.00 \pm 0.02$ & $0.01 \pm 0.02$ & $0.08 \pm 0.02$ & 0.00 & \pm & 0.03 & $-0.08 \pm 0.03$ & $\mathrm{~m}$ \\
\hline & $@ 1.5 \mathrm{~m} / \mathrm{s}$ & $0.05 \pm 0.04$ & $0.07 \pm 0.04$ & $0.05 \pm 0.04$ & -0.02 & \pm & 0.06 & $0.02 \pm 0.06$ & $\mathrm{~m}$ \\
\hline
\end{tabular}

The two $1 \mathrm{~g}$ fits above show a trend of increasing vertical displacement of the hip with gait speed, while the lunar gravity fit shows a slightly decreasing displacement of the hip with gait speed. These observations match well with the results for hip angle, discussed above. 
As with hip angle, the constrained geometry of the walking gait requires that in order to increase gait speed by lengthening the stride, the hip must dip lower at toe-off/heel strike.

In a purely running gait, on the other hand, forward distance is gained in a series of ballistic flights. In order to make each step both shorter in duration and longer in distance, the runner must "launch" each step at a greater speed and a shallower angle from the horizontal. This shallower trajectory results in less vertical motion of the hip.

The data indicate a significant difference in intercept between $1 g$ and lunar underwater environments. At the typical walking speed, however, the redimensionalized difference in hip motion is slight in both dry vs. wet and $1 g$ vs. lunar comparisons. 


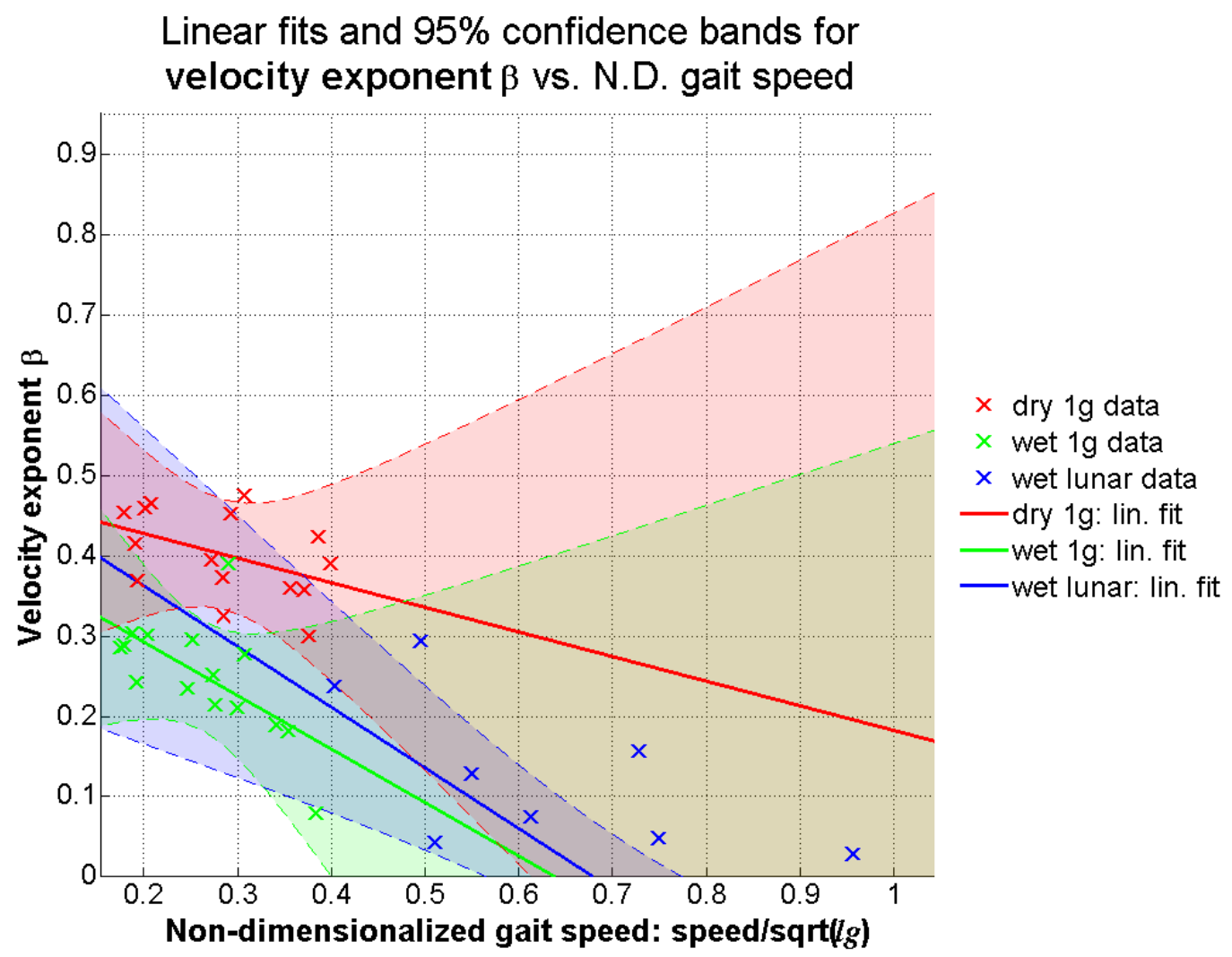

Figure 17: Velocity exponent vs. gait speed

\begin{tabular}{|c|c|c|c|c|c|c|c|c|c|c|}
\hline$\underline{\text { Metric }}$ & Parameter & dry $1 g$ & wet $1 \mathrm{~g}$ & wet lunar & $\frac{\text { dry vs. wet }}{(95 \%)}$ & $\begin{array}{c}\text { Reject } \\
\text { H0? }\end{array}$ & \multicolumn{3}{|c|}{$\frac{1 \mathrm{~g} \text { vs. lunar }}{(95 \%)}$} & $\begin{array}{c}\text { Reject } \\
\underline{\text { H0? }}\end{array}$ \\
\hline velocity & slope & $-0.31 \pm 0.35$ & $-0.67 \pm 0.42$ & $-0.76 \pm 0.14$ & $0.36 \pm 1.33$ & - & 0.09 & \pm & 1.07 & - \\
\hline exponent $(\beta)$ & intercept & $0.49 \pm 0.11$ & $0.43 \pm 0.11$ & $0.51 \pm 0.10$ & $0.06 \pm 0.38$ & - & -0.09 & \pm & 0.38 & - \\
\hline
\end{tabular}

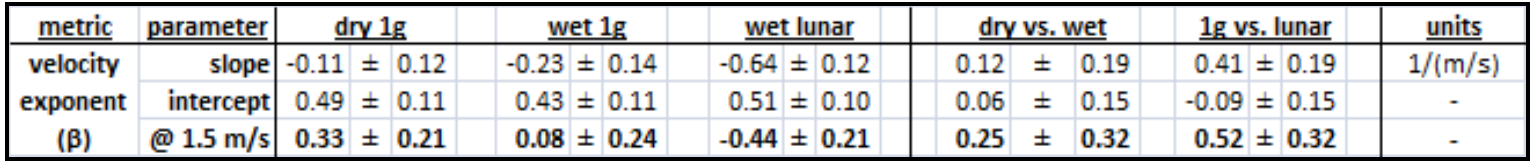

These three plots indicate a trend of decreasing velocity exponent with increasing gait speed, matching expectations. ${ }^{[10]}$ The null hypothesis is not rejected in any of the coefficient comparisons. The slope for the dry $1 \mathrm{~g}$ fit is shallower than those for the 
wet environments; however, the confidence bands for the $1 g$ fits diverge sharply, indicating the large error associated with the slope estimates.

At $1.5 \mathrm{~m} / \mathrm{s}$, the difference in non-dimensional $\beta$ value between dry and wet $1 \mathrm{~g}$ environments is within the margin of error, with $\beta$ expected to be greater in the dry environment by a difference of $0.25 \pm 0.32$. The difference between $1 g$ and lunar gravity underwater environments is twice as large, with $\beta$ expected to be greater in the $1 \mathrm{~g}$ environment by $0.52 \pm 0.32$.

\section{Summary of gait comparison results}

In comparing dynamical gait metrics between dry and wet $1 g$ environments and between $1 \mathrm{~g}$-ballasted and lunar gravity-ballasted underwater environments, the following results were established.

In all environments, gait frequency increases with gait speed, although it does so at a significantly slower rate in lunar gravity than in $1 \mathrm{~g}$. Step length also increases with gait speed, and is comprable for all three environments, although at a typical gait speed of $1.5 \mathrm{~m} / \mathrm{s}$, step lengths are somewhat longer in lunar gravity. Maximum hip angle increases with gait speed in all three environments, although it does so at a significantly slower rate in lunar gravity than in $1 \mathrm{~g}$. At typical gait speed, maximum hip angle is approximately 0.4 radians smaller in $1 \mathrm{~g}$ than in lunar gravity. 
Vertical displacement of the torso tends to increase with gait speed in $1 \mathrm{~g}$ environments over the range of gait speeds tested, while torso displacement tends to decrease slightly with gait speed in lunar gravity. At typical gait speed, hip displacement is comparable in all three environments.

Velocity exponent $\beta$ tends to decrease with gait speed, although it does so at a shallower rate in the dry environment than in the underwater environments.

Observations on the trend of each metric with respect to gait speed were consistent with prior research.

True lunar environment gait metric estimates

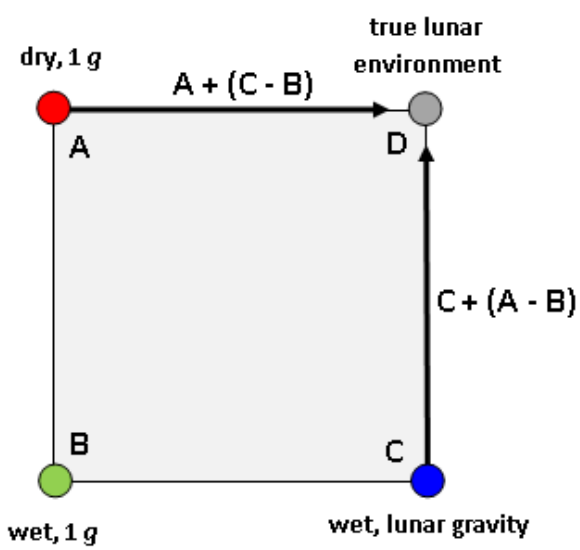

Figure 18: Motion-capture data design matrix

As a secondary analysis, the data collected via motion-capture may be used to generate estimates for expected gait metrics in true lunar gravity, rather than that of the underwater ballasted environment. Taking the assumption that a given difference 
in gravitational acceleration will have the same effect on gait dynamics in dry and underwater environments - or, conversely, taking the assumption that the difference between dry and underwater environments is the same regardless of gravitational acceleration - one can easily calculate the expected value of a given metric in the true lunar environment, as illustrated in Figure 18: Motion-capture data design matrix above. This process is essentially a vector addition of the affect of one environmental variable - either gravitational acceleration or the underwater environment - to an environment in which the other variable is present. In other words, data for the dry, 1 $g$ environment (A) are offset by the difference (C-B) between $1 g$ and lunar environments, in order to estimate data for the true lunar environment (D). Equivalently, data for the underwater, lunar gravity environment $(C)$ are offset by the difference (A-B) between wet and dry environments, to generate the same result for the true lunar environment (D).

Taking $A, B$, and $C$ to be metric values at a given non-dimensionalized gait speed, $D=A+C-B$ yields an estimate for that metric in the lunar environment. Alternately, $A, B$, and $C$ may be the coefficients of regression fits relating metric values to gait speeds in each tested environment.

Applying this assumption to the gait metric data analyzed above, estimates are generated for non-dimensionalized stride frequency, step length, maximum hip angle, vertical torso displacement, and $\beta$ as a function of non-dimensionalized gait speed in the true lunar environment: 


\section{Estimates for gait metric functions in true lunar gravity}

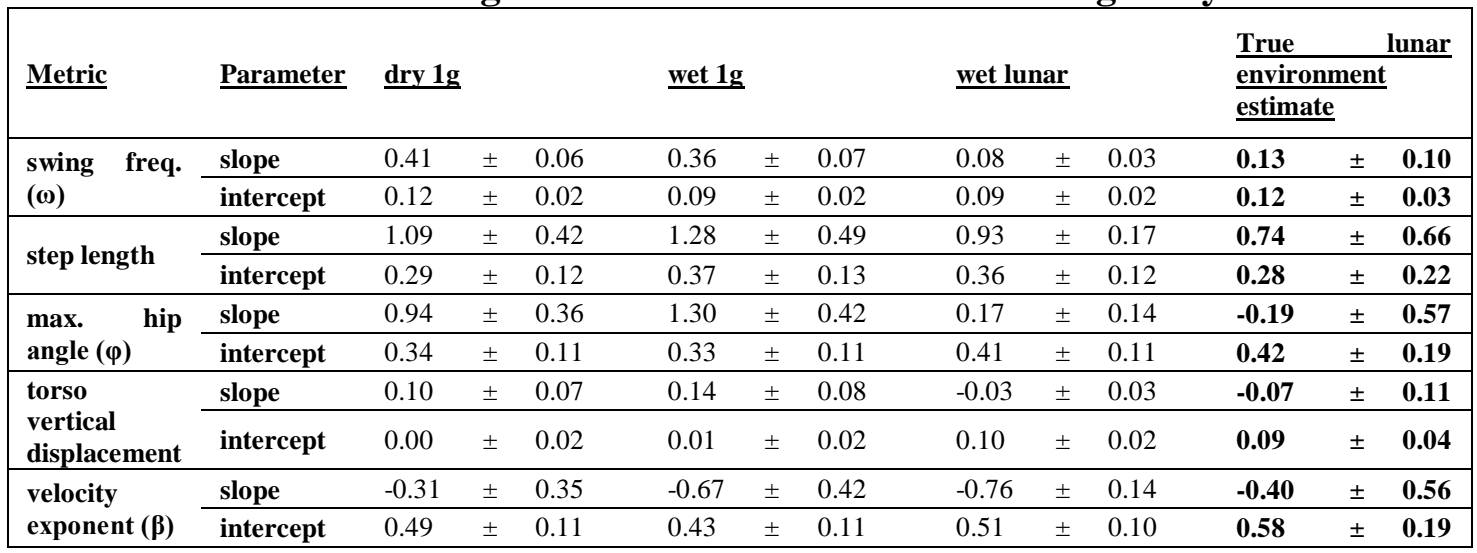

Looking at the table, it is apparent that the error values are close to, and in some cases larger than, the parameter estimates. This is due to the compounding of error from the three test environments; the error for the true lunar environment metric function is taken to be the 2-norm - or the square root of the sum of the squares - of the errors from the three physical test environments, as is the standard method for compounding experimental error in a sum or difference of independent quantities. In order to generate reliable gait metric functions for the true lunar gravity environment by this method, the experimental error must be reduced, either by increasing the sample size (number of subjects), working to reduce measurement error, or the like.

That said, it is interesting to note the apparent negative slope of maximum hip angle as a function of gait speed. In each of the three test environments, this trend was positive; however, the slope was greatest for the $1 \mathrm{~g}$, underwater test environment, indicating that both increased gravity and underwater affects contributed to a positive trend. With these effects removed, the expected trend in the true lunar environment is in fact a negative one, albeit well within the margin of error. 
The expected sign of the trends for swing frequency, step length, and velocity exponent with gait speed all match the trends seen in the physical test data. ${ }^{[9][10][13][17]}$ In the case of vertical displacement of the torso, the trend in the true lunar environment is expected to be negative, as was seen in the lunar-gravity, underwater environment.

The following plots show the estimated gait metric functions for the true lunar environment, along with $95 \%$ confidence bands, calculated using the method described above. Due to the compounding of error, the confidence bands for these estimated functions are wider than the confidence bands for the physical test environments.

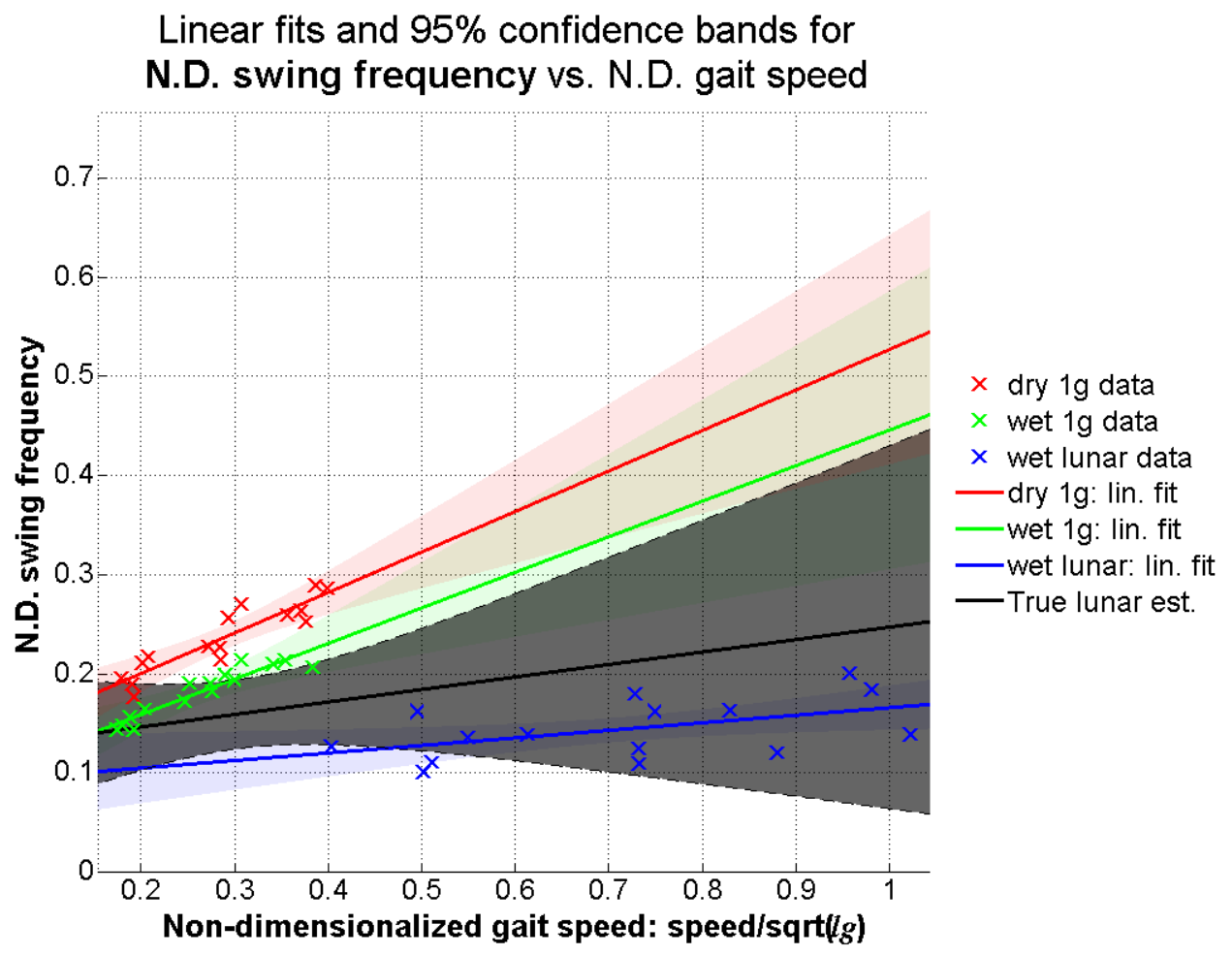

Figure 19: Stride frequency in the true lunar environment 
Linear fits and 95\% confidence bands for

N.D. step length vs. N.D. gait speed

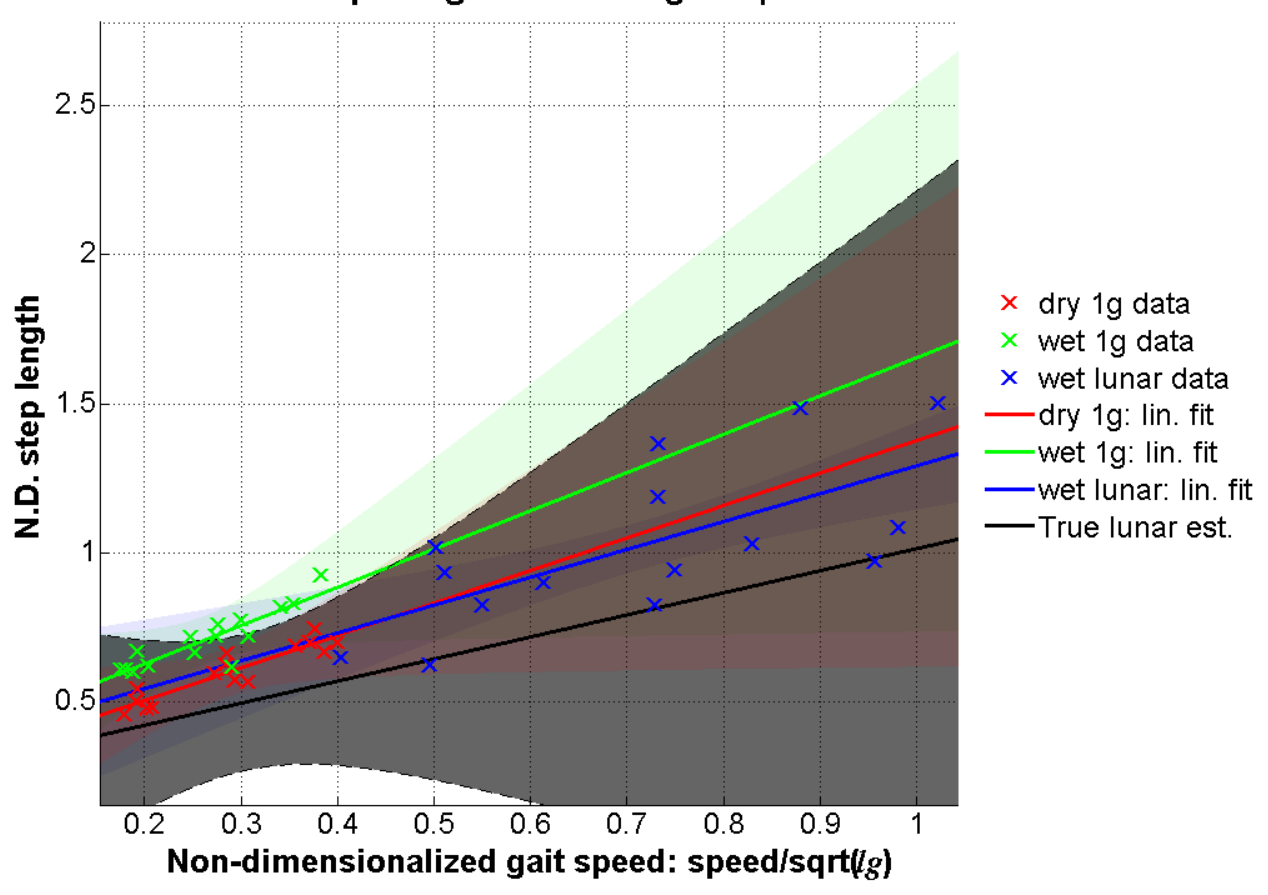

Figure 20: Step length in the true lunar environment

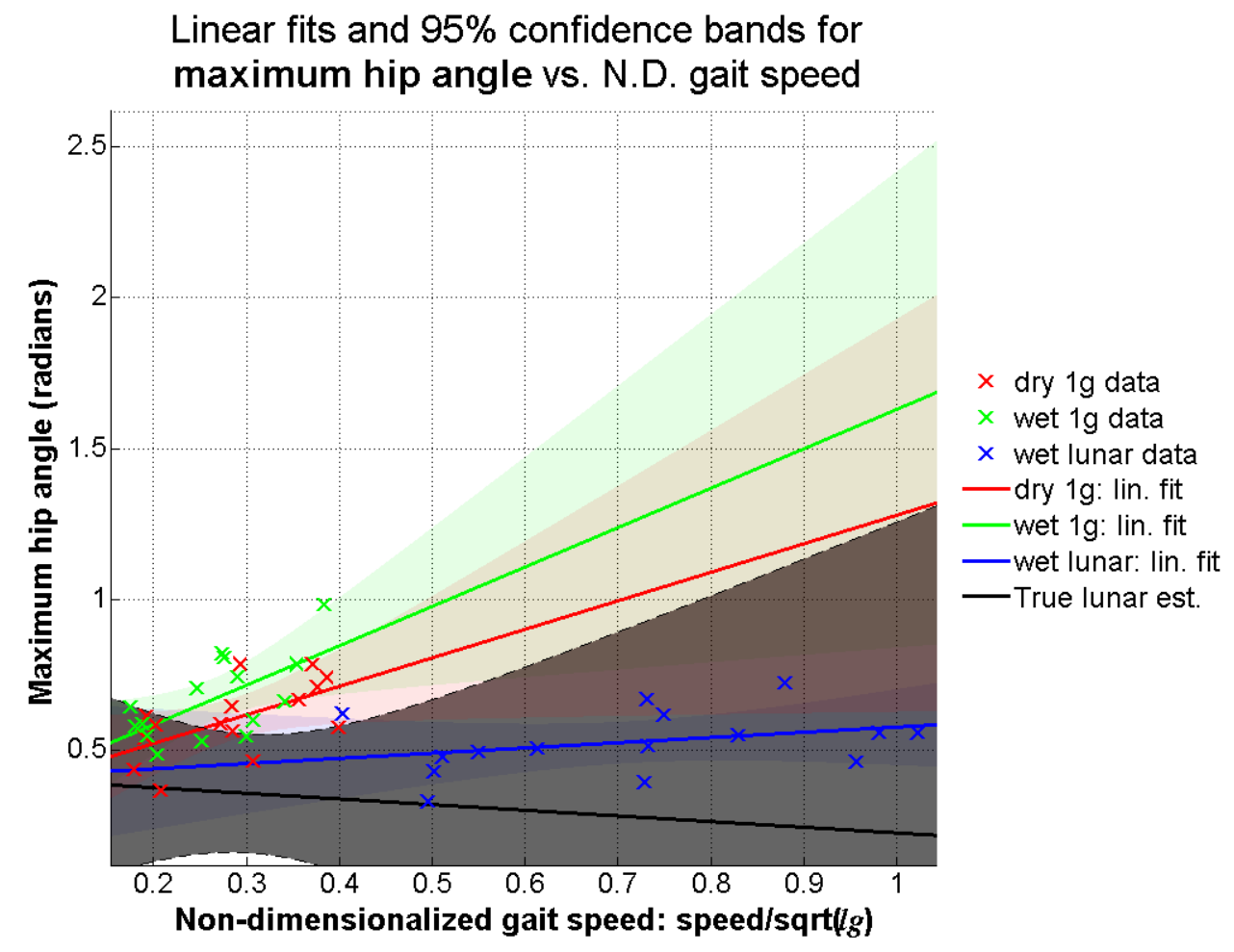

Figure 21: Maximum hip angle in the true lunar environment 
Linear fits and $95 \%$ confidence bands for N.D. torso vertical displacementvs. N.D. gait speed

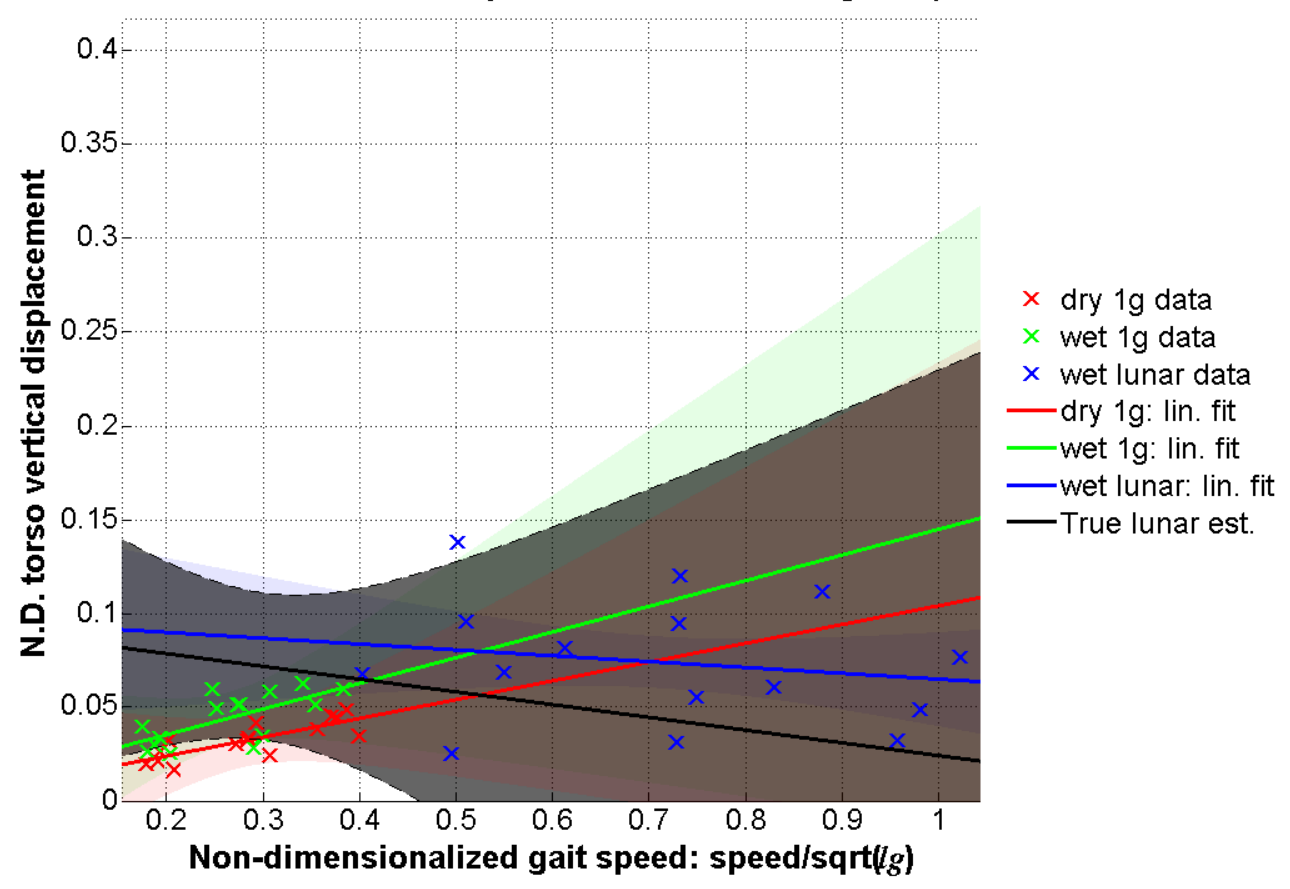

Figure 22: Vertical displacement of the torso in the true lunar environment

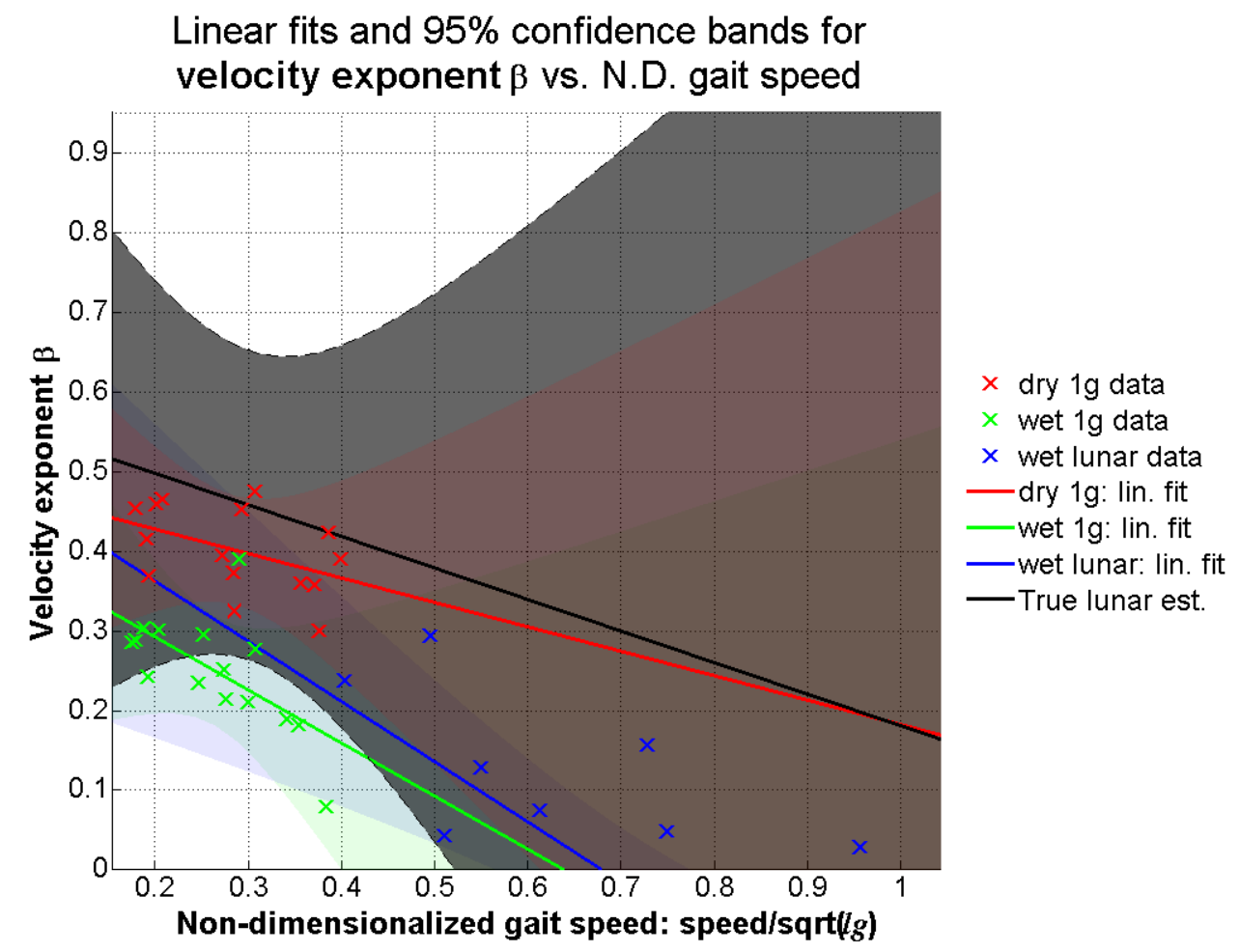

Figure 23: Velocity exponent in the true lunar environment 


\section{Chapter 7: Motion-Capture Testing Conclusions}

A clear walk-run transition regime was observed in terms of the non-dimensional Froude number, enabling prediction of gait type given gravitational acceleration and subject leg length only.

In most cases, the difference in dynamical gait metrics between $1 g$ and lunar gravity environments was significantly larger than the differences between dry and underwater environments. The markedly different behavior of gaits in lunar gravity is likely attributable to a difference in gait type, with the walk-run transition occuring at slower absolute gait speeds in reduced-gravity environments. ${ }^{[8]}$

Because of the similarity of results in wet and dry environments, the lunar-gravity underwater environment was the best analog, among the three physical test environments, of the true lunar environment, and is a quantitatively better predictor of gait metrics in true lunar gravity than a dry, $1 \mathrm{~g}$ environment.

Given these results, it is apparent that the difference in gravitational acceleration between terrestrial and lunar environments has a much more significant impact on gait dynamics than the difference between dry and underwater environments, when gravitational acceleration is controlled by ballasting. This implies that ballasted underwater gait testing is a useful means for approximating true lunar gait dynamics. 
Ballasted underwater partial gravity simulation presents a means of achieving a higher-fidelity approximation of true lunar gait dynamics than is possible in a dry, $1 \mathrm{~g}$ environment. Ballasted underwater partial gravity simulation is, and will continue to be, a vital tool in the study of reduced gravity gaits, especially where alterate means of partial gravity simulation, i.e. suspension rigs and parabolic flight, are unavailable or impractical to apply. 


\section{Chapter 8: Extended Test Matrix: Dynamic Walker/Runner Models}

As a secondary, supplemental analysis to the comparisons performed between gait metrics measured in physical testing by means of motion capture, a pair of dynamic gait models were created to assess the ability of simple dynamic models to capture the behavior of corresponding physical environments. These models attempted to replicate various gait metrics of the real, recorded gaits. The models analyzed below are extensions of the Simplest Model and Anthropomorphic Model described by Kuo for the walking model, and the impulsive runner described by McGeer for the running model. $^{[9][10][5][13][12]}$

The combination of physical motion-capture data and simulated gait models allow for estimation of gait metrics for real reduced-gravity environments using multiple approaches, which may be compared and combined. A simplified test matrix is visualized below. The matrix has three dimensions of comparison: physical test data vs. virtual models, drag-free environments vs. the underwater environment, and $1 \mathrm{~g}$ vs. $1 / 6^{\text {th }} g$ environments. The vertex labeled $F$ represents true lunar gait, the unknown which we would like to describe using knowledge about gait metrics in experimental environments, which are represented by the other vertices.

Given the multidimensionality of the test matrix, gait metrics for the lunar surface can be estimated by several approaches, and the context provided by these different 
approaches allows one to quantify the fidelity or closeness of different test gaits to true lunar gait. For a given "face" of the test matrix that includes $F$, it is apparent that information about gaits at the other three vertices may yield information about gaits at $F$. For example, knowing the value of some gait metric for vertex $E$ (underwater ballasted simulation of lunar gravity), vertex $A$ (a computer model of gait in the same environment), and vertex $B$ (a computer model of drag-free gait in lunar gravity), one can estimate the value of the metric at $F$ as: (value at $F)=($ value at $E)+($ value at $B)$ - (value at $A$ ). This approach assumes that the dimensions of the matrix are independent - that the effect had by one dimension on a gait metric is not affected by another dimension. This is not necessarily a valid assumption; intuitively, the difference in stride frequency between underwater and drag-free environments is likely to be larger in $1 \mathrm{~g}$ than in lunar gravity. However, in the absence of detailed knowledge of these dependencies, the assumption of independence is a necessary one. By comparing estimates from each cube "face", the estimates of gait metrics in true gravity can be refined. 


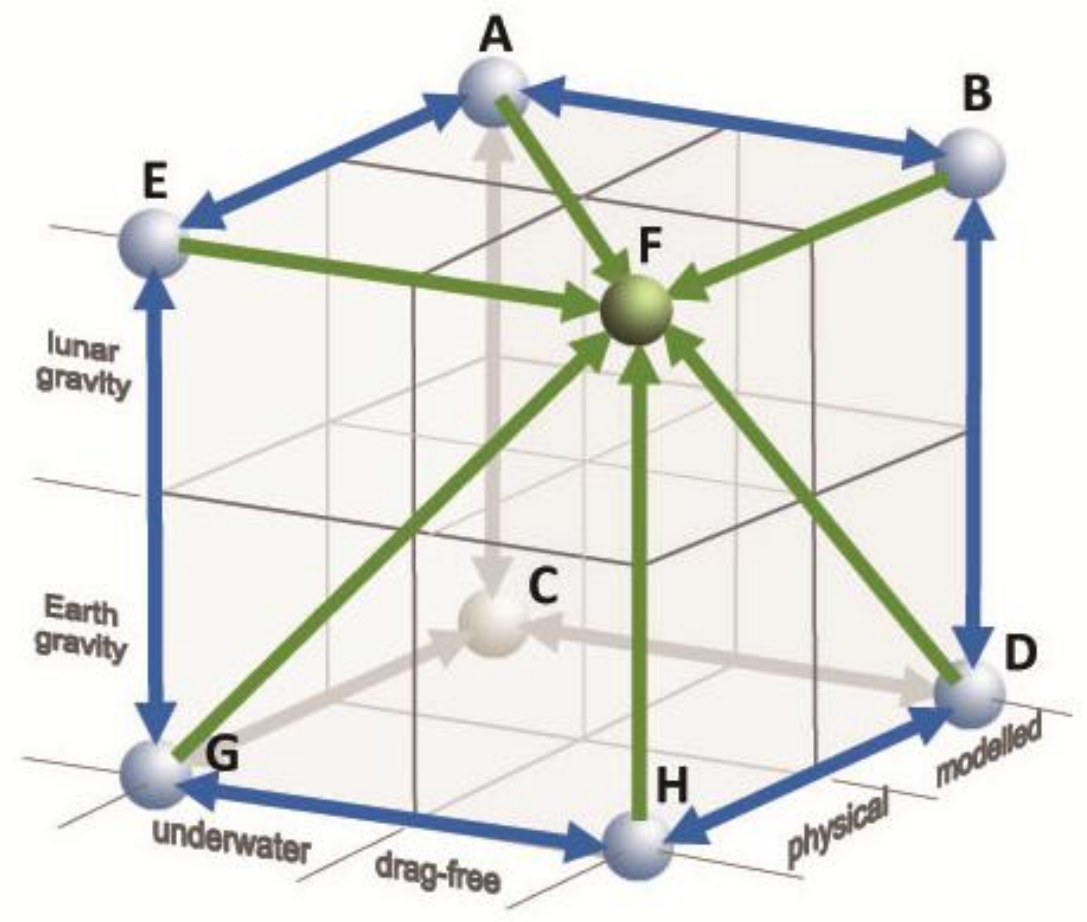

Figure 24: Visualization of extended test matrix

In addition to estimating gait metrics in true lunar gravity, the test matrix can be used to generate multiple estimates of the magnitude of the effect that a given dimension has on the value a given metric. By taking the difference in the metric along opposite edges of the matrix and averaging them, a reasonable estimate for the size of the effect can be found. For example, the effect that gravity has on a given metric may be estimated as: (effect of reduced gravity on metric $)=$ average $[$ (value at $\mathrm{E})-($ value at $\mathrm{G})$, (value at B) - (value at D) ]. This approach is a compromise between an estimate along the $E G$ edge, which is based on physical data but may be skewed by the presence of drag, and an estimate along the $B D$ edge, which is free from drag-induced distortion but may be inaccurate due to shortcomings of the design of the model. 
Note that most directly-measured gait metrics do not have a single value for a given environment, but vary interdependently. For the analyses which follow, walking/running speed (relative to the walking/running surface) is considered the independent variable on which measured (and computed) gait metrics depend.

Using these methods and the data collected from the physical and virtual gait analyses, gait in the lunar environment is characterized, and the effects of gravity, drag, and gait modeling limitations on gait are assessed.

\section{Simple gait models in the literature}

Kuo $^{[10]}$ presents three simple gait models of increasing realism and computational complexity, each of which are presented briefly here. Each is a two-dimensional, twolink model with rigid legs; i.e., knee and ankle joints are absent. In order to replicate the role of knee-bending in human gait, the swing leg is cleared to pass the stance leg in mid-stride. In each model, the mass of the upper body is reduced to a point mass at the hip.

Although McGeer showed that a passive walker can recover losses by walking on a downward slope ${ }^{[13][12]}$, Kuo specifies the use of an impulsive toe-off just prior to heel strike as the primary energy input to the walkers. Kuo uses a torsional spring at the hip to tune the swing frequency of each walker, in order to minimize collision losses, and thus the required energy input at each toe-off. 
Kuo's "Idealized Simplest Model" (ISM), based on Garcia, ${ }^{[5]}$ uses a small-angle approximation for the angle of each leg from the vertical. The model has massless legs, and point mass feet with mass much less than that of the upper body. These linearizing simplifications allow for an analytical solution to the equations of motion.

The "Simplest Model" (SM) presented by Kuo does away with the small-angle approximation of the idealized model, and does not assume that the foot masses are significantly less than the torso mass.

Finally, the "Anthropomorphic Model" includes evenly-distributed leg masses, rather than point masses at the feet, and has circularly-curved, rather than point, feet. As introduced by McGeer ${ }^{[13]}$, the curved feet mitigate collisional losses at heel strike, and result in gait dynamics somewhere between that of a pure inverted pendulum, and the "synthetic wheel" used by McGeer to introduce basic concepts of gait.

The virtual gait models used in this study incorporate features from Kuo's Simplest Model and Anthropomorphic Model; distributed leg masses are used, although point feet, rather than curved feet, are used. The primary modification of these models is the addition of a drag force on each leg to attempt to capture some of the affects of gait in the underwater environment. The derivation of the models used in this study is presented below. 


\section{Qualitative description of gait models}

Passive Dynamic Walker
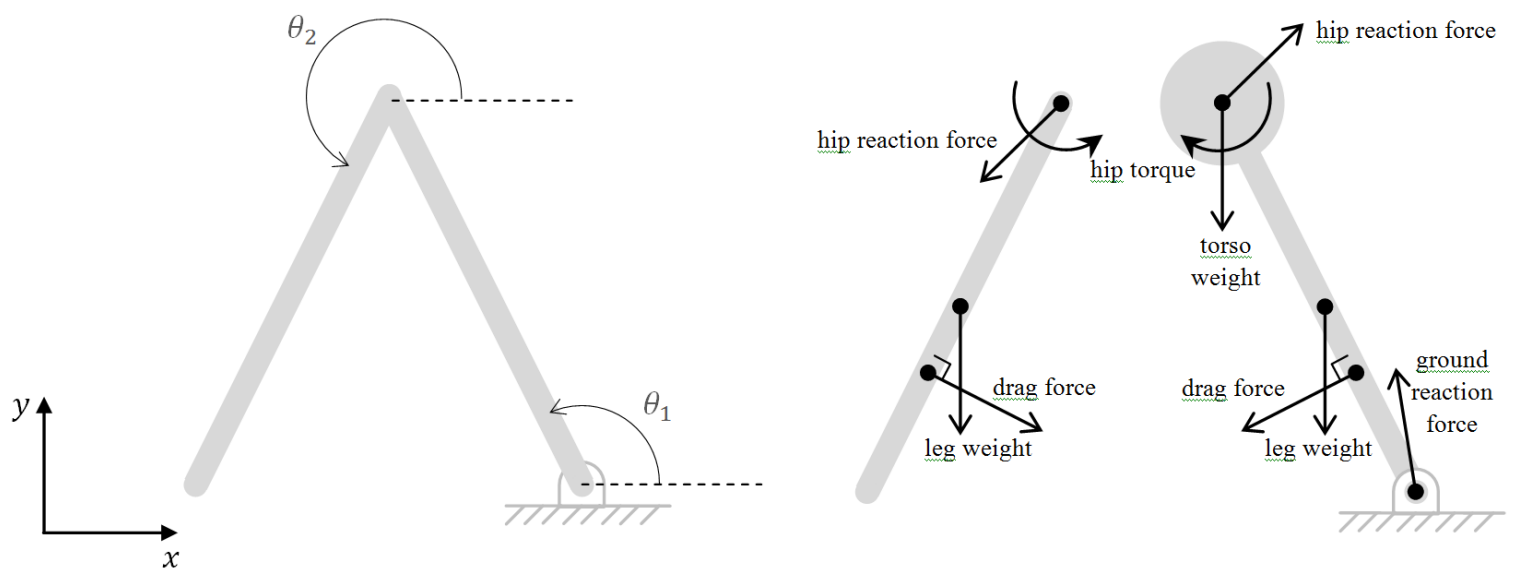

Figure 25: Definition of leg angles and coordinate frame (left) and free body diagram (right) for the walking case

The passive dynamic walker model used herein consists of two rigid (knee-less) legs with identical length and evenly-distributed masses, attached at the hip via a torsional spring. The walking surface is assumed to be zero-slip, and the stance foot (the foot in contact with the ground) is constrained to a fixed position. This constraint is realized by a ground reaction force applied to the stance foot, though it is not necessary to calculate this force in order to solve the dynamics of the walker. McGeer notes that the dynamics of knee-less models do not differ appreciably from those of more complex models with knee articulation. ${ }^{[13]}$

In addition to the masses of the legs, a torso mass is attached to the hip of the stance leg, or in the running case where both legs are off the ground, the forward leg. (The 
choice of which leg to attach the torso mass to is arbitrary, and mathematically irrelevant.) The ratio of leg mass to torso mass is based on the anthropometry of $50^{\text {th }}$ percentile adults. ${ }^{[1]]}$

In the walking gait, exactly one foot remains in contact with the ground at all times, with inelastic heel-strike and impulsive toe-off occurring at the same instant. The walking gait may be divided into two symmetrical phases, each with one foot planted and the other swinging, and may thus be described by a single set of equations, with a single impulsive transfer (and, in the non-conservative case, addition) of energy at each heel-strike/toe-off.

The legs are attached at the hip via a torsional spring, which tends to pull the legs together. In past research into the efficiency of passive dynamic walkers, it has been found that including such a spring, and tuning the spring constant so as to minimize the energy expenditure per unit distance, provides a more accurate model of human biomechanics than a passive dynamic walker without such a spring. (For the purpose of calculating this energy expenditure, the hip spring is assumed to recover stored energy with perfect efficiency, and the only input of energy to the system is the impulsive toe-off. $)^{[10]}$

In order to model drag underwater, a drag force is applied perpendicular to each leg. The legs are assumed to present a uniform cross-section; i.e., a non-rotating leg experiences zero drag moment about its geometric center. Because the walker is 
assumed to be on a treadmill belt which is moving backward at the same average speed that the walker moves forward, torso motion in the horizontal direction is assumed to be negligible, and drag on the torso is neglected.

It is interesting to note that the passive dynamic walker is effectively a double pendulum, with the stance foot as the first pivot point and the hip as the second, albeit with the addition of hip torque and drag forces. ${ }^{[5]}$

Symmetrical Impulsive Runner
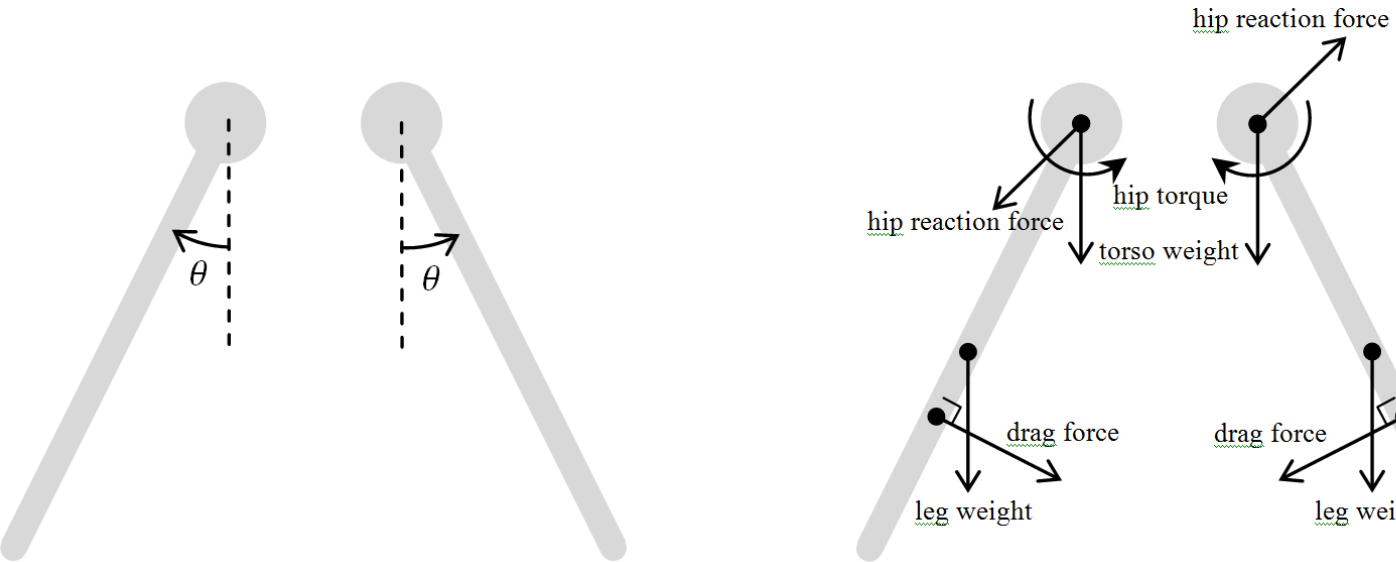

Figure 26: Definition of leg angles (left) and forces and free-body diagrams (right)

For the running case, a symmetrical impulsive "bounce" model is used, based on McGeer's passive dynamic runner, whose motion is described as "essentially bouncing and scissoring in synchronicity." ${ }^{[12]}$ Contact with the ground is assumed to be an instantaneous, elastic collision at the point of cross-over of the two legs (the point at which the front leg becomes the rear, and vice versa). Energy is added at this collision in the form of a vertical impulse and an impulsive hip torque, which 
counteract the energy lost over each step due to drag. Because the model represents a subject on a treadmill, with the subject's forward motion and the treadmill's rearward motion exactly cancelling out, there should be no net horizontal velocity relative to the surrounding medium; therefore, the magnitude and the moment are of the drag force on each leg are assumed to be identical. The direction of the drag force is defined such that an increasing $\theta$ or $y_{C M}$ creates a positive drag.

Because the angles of the legs from the vertical are equal and horizontal movement of the system is constrained to zero, the system comprises two degrees of freedom, which may be expressed as the angle each leg makes with the vertical, and the vertical position of the center of mass of the system. The equations of motion for the symmetrical impulsive runner are stated as follows.

Newton-Euler equations

The equations of motion for each gait are derived starting from the familiar vector form of the Newton-Euler equations, with masses and mass moments of inertia constant. For a body $i$, Newton's equation takes the form:

$$
m_{i} \boldsymbol{a}_{i}=\sum \boldsymbol{f}_{i},
$$

With $m_{i}, \boldsymbol{a}_{i}$ and $\sum \boldsymbol{f}_{i}$ representing the mass of the body, the linear acceleration vector of the body, and the vector sum of the forces acting on the body, respectively. Likewise, Euler's equation takes the form: 


$$
{ }^{x} I_{i} \boldsymbol{\alpha}_{i}=\sum{ }^{x} m_{i}
$$

With ${ }^{x} I_{i}, \alpha_{i}$ and $\sum{ }^{x} m_{i}$ representing, respectively, the mass moment of inertia of the body with respect to a given station $x$, the angular acceleration vector of the body, and the vector sum of the moments acting on the body with respect to station $x$. 


\section{Chapter 9: Derivations of Equations of Motion for Passive Dynamic Walker}

\section{Definition of constants and variables}

The constants and variables defined below will be substituted into the equations of motion, which are derived in the following section. Note the use of shorthand notation $c_{i}=\cos \left(\theta_{i}\right)$ and $s_{i}=\sin \left(\theta_{i}\right)$. Note also that the subscript one refers to either the stance leg (in the case where one foot is on the ground) or the forward leg (in the case where both feet are off the ground). Likewise, the subscript 2 refers to either the swing leg (in the case where one foot is on the ground) or the rear leg (in the case where both feet are off the ground). When used as left-hand superscripts, 1 and 2 refer to the reference frame of the foot of the corresponding leg.

\section{Constants}

$g \quad$ acceleration of gravity in the specified environment (either terrestrial or lunar)

$k \quad$ torsional spring constant of the hip

$L \quad$ length of each leg

$m \quad$ mass of each leg

M mass of the torso

${ }^{1} I_{1} \quad$ moment of inertia of the stance leg plus torso, about the stance foot:

$$
{ }^{1} I_{1}=\left(\frac{m L^{2}}{3}+M L^{2}\right)
$$

${ }^{h i p} I_{2} \quad$ moment of inertia of the swing leg about its center of mass:

$$
{ }^{h i p} I_{2}=\frac{m L^{2}}{3}
$$


Position, velocity, and acceleration vectors for the geometric centers and centers of mass

Because the mass of each leg is evenly distributed, its center of mass is at its geometric center. By geometry, the positions of the geometric centers of each leg with respect to the stance foot are as follows:

$$
{ }^{1} \boldsymbol{p}_{\text {mid }_{1}}=\left[\begin{array}{c}
\frac{L}{2} c_{1} \\
\frac{L}{2} s_{1} \\
0
\end{array}\right] \quad{ }^{1} \boldsymbol{p}_{\text {mid }_{2}}=\left[\begin{array}{c}
L c_{1}+\frac{L}{2} c_{2} \\
L s_{1}+\frac{L}{2} S_{2} \\
0
\end{array}\right]
$$

Taking the time-derivative of each position vector yields velocity vectors relative to the stance foot:

$$
{ }^{1} v_{\text {mid }_{1}}=\left[\begin{array}{c}
-\frac{L}{2} \dot{q}_{1} s_{1} \\
\frac{L}{2} \dot{q}_{1} c_{1} \\
0
\end{array}\right] \quad{ }^{1} v_{\text {mid }_{2}}=\left[\begin{array}{c}
-L \dot{q}_{1} s_{1}-\frac{L}{2} \dot{q}_{2} s_{2} \\
L \dot{q}_{1} c_{1}+\frac{L}{2} \dot{q}_{2} c_{2} \\
0
\end{array}\right]
$$

Finally, taking the time-derivative of each velocity vector yields acceleration vectors for the center of each leg:

$$
\boldsymbol{a}_{\text {mid }_{1}}=\left[\begin{array}{c}
-\frac{L}{2} \ddot{q}_{1} s_{1}-\frac{L}{2} \dot{q}_{1}^{2} c_{1} \\
\frac{L}{2} \ddot{q}_{1} c_{1}-\frac{L}{2} \dot{q}_{1}^{2} s_{1} \\
0
\end{array}\right] \quad \boldsymbol{a}_{m i d_{2}}=\left[\begin{array}{c}
-L \ddot{q}_{1} s_{1}-L \dot{q}_{1}^{2} c_{1}-\frac{L}{2} \ddot{q}_{2} s_{2}-\frac{L}{2} \dot{q}_{2}^{2} c_{2} \\
L \ddot{q}_{1} c_{1}-L \dot{q}_{1}^{2} s_{1}+\frac{L}{2} \ddot{q}_{2} c_{2}-\frac{L}{2} \dot{q}_{2}^{2} s_{2} \\
0
\end{array}\right]
$$


Finally, two more position vectors are defined, denoting the position of the hip with respect to the stance foot and the geometric center of the swing leg with respect to the hip, respectively:

$$
{ }^{1} p_{\text {hip }}=\left[\begin{array}{c}
L c_{1} \\
L s_{1} \\
0
\end{array}\right] \quad{ }^{\text {hip }} \boldsymbol{p}_{\text {mid }}=\left[\begin{array}{c}
\frac{L}{\frac{L}{2}} c_{2} \\
\frac{L}{2} S_{2} \\
0
\end{array}\right]
$$

Finally, the angular accelerations of the two legs, and the forces and torques acting on the legs, must be defined:

Angular accelerations

$$
\begin{array}{lll}
\underline{\text { Vector }} & & \text { Definition } \\
\alpha_{1} & =\left[\begin{array}{c}
0 \\
0 \\
\ddot{q}_{1}
\end{array}\right] & \text { angular acceleration of the stance leg (leg 1) } \\
\alpha_{2} & =\left[\begin{array}{c}
0 \\
0 \\
\ddot{q}_{2}
\end{array}\right] & \text { angular acceleration of the swing leg (leg 2) }
\end{array}
$$

\section{Definition}

Forces
Vector

$$
\begin{array}{ll}
\boldsymbol{d}_{1} & =\left[\begin{array}{c}
-d_{1} s_{1} \\
d_{1} c_{1} \\
0
\end{array}\right] \\
\boldsymbol{d}_{2} & =\left[\begin{array}{c}
-d_{2} s_{2} \\
d_{2} c_{2} \\
0
\end{array}\right] \\
\boldsymbol{f}_{2 \rightarrow 1} & =\left[\begin{array}{c}
f_{2 \rightarrow 1_{x}} \\
f_{2 \rightarrow 1_{y}} \\
0
\end{array}\right]= \\
\boldsymbol{g}_{\text {leg }} & =\left[\begin{array}{c}
0 \\
-m g \\
0
\end{array}\right]
\end{array}
$$$$
\boldsymbol{f}_{2 \rightarrow 1} \quad=\left[\begin{array}{c}
f_{2 \rightarrow 1_{x}} \\
f_{2 \rightarrow 1_{y}} \\
0
\end{array}\right]=-\boldsymbol{f}_{1 \rightarrow 2} \text { force applied at the hip by the swing leg (leg 2) on }
$$

\section{Definition}

drag force on the stance leg (leg 1), where $d_{1}$ is defined later

drag force on the swing leg (leg 2)

gravitational force on each leg 
$\boldsymbol{g}_{\text {torso }} \quad=\left[\begin{array}{c}0 \\ -M g \\ 0\end{array}\right] \quad$ gravitational force on the torso

Torques

$$
\begin{array}{ll}
{ }^{1} n_{1} & =\left[\begin{array}{c}
0 \\
0 \\
n_{1}
\end{array}\right] \\
{ }^{m i d} n_{2} & =\left[\begin{array}{c}
0 \\
0 \\
n_{2}
\end{array}\right] \\
\boldsymbol{\tau}_{h} & {\left[\begin{array}{c}
\text { Moment induced by drag on the stance leg, about } \\
\text { the stance foot, where } n_{1} \text { is defined later } \\
0 \\
0 \\
k\left(\theta_{1}-\theta_{2}+\pi\right)
\end{array}\right] \begin{array}{r}
\text { Moment induced by drag on the swing leg, about } \\
\text { its geometric center }
\end{array}}
\end{array}
$$

\section{Equations of motion for the walking gait}

Because the stance foot is geometrically constrained to a fixed position, the system has only two degrees of freedom, which may be expressed as the angles made by each leg with the horizontal. The Newton-Euler equations yield four scalar differential equations in terms of the unknown components of the hip reaction force. By applying the geometric constraint that the legs are joined at the hip, these unknown force components can be eliminated from the equations, and the system of equations reduced to the necessary two.

Stance Leg (Leg 1)

Euler's equation is applied to the stance leg about the stance foot, thus eliminating the ground reaction force at the stance foot from the equation. Because the motion of the 
stance leg is constrained such that the stance foot does not move, its motion can be described completely by applying Euler's equation alone:

$$
\begin{gathered}
{ }^{1} I_{1} \boldsymbol{\alpha}_{1}=\underbrace{\left({ }^{1} \boldsymbol{p}_{\text {hip }} \times \boldsymbol{f}_{2 \rightarrow 1}\right)}_{\text {Hip reaction moment }}+\underbrace{\left({ }^{1} \boldsymbol{p}_{\text {mid }} \times \boldsymbol{g}_{\text {leg }}\right)}_{\text {Gravitational moment }}+\underbrace{\left({ }^{1} \boldsymbol{p}_{\text {hip }} \times \boldsymbol{g}_{\text {torso }}\right)}_{\text {Gravitational moment }}+\underbrace{\boldsymbol{n}_{1}}_{\begin{array}{c}
\text { Drag } \\
\text { moment }
\end{array}} \\
-\underbrace{\boldsymbol{\tau}_{h}}_{\begin{array}{c}
\text { Hip } \\
\text { torque }
\end{array}}
\end{gathered}
$$

Expanding the vectors:

$$
\begin{aligned}
& { }^{1} I_{1}\left[\begin{array}{c}
0 \\
0 \\
\ddot{q}_{1}
\end{array}\right]=\left(\left[\begin{array}{c}
L c_{1} \\
L s_{1} \\
0
\end{array}\right] \times\left[\begin{array}{c}
f_{2 \rightarrow 1_{x}} \\
f_{2 \rightarrow 1_{y}} \\
0
\end{array}\right]\right)+\left(\left[\begin{array}{c}
\frac{L}{2} c_{1} \\
\frac{L}{2} s_{1} \\
0
\end{array}\right] \times\left[\begin{array}{c}
0 \\
-m g \\
0
\end{array}\right]\right)+\left(\left[\begin{array}{c}
L c_{1} \\
L s_{1} \\
0
\end{array}\right] \times\left[\begin{array}{c}
0 \\
-M g \\
0
\end{array}\right]\right)+n_{1} \widehat{\boldsymbol{k}}- \\
& \tau_{h} \widehat{\boldsymbol{k}}
\end{aligned}
$$

Calculating the cross products:

$$
\begin{aligned}
& { }^{1} I_{1}\left[\begin{array}{c}
0 \\
0 \\
\ddot{q}_{1}
\end{array}\right]=\left(\left[\begin{array}{c}
0 \\
0 \\
L c_{1} f_{2 \rightarrow 1_{y}}-L s_{1} f_{2 \rightarrow 1_{x}}
\end{array}\right]\right)+\left(\left[\begin{array}{c}
0 \\
0 \\
-\frac{L}{2} c_{1} m g
\end{array}\right]\right)+\left(\left[\begin{array}{c}
0 \\
0 \\
-L c_{1} M g
\end{array}\right]\right)+n_{1} \widehat{\boldsymbol{k}}- \\
& \tau_{h} \widehat{\boldsymbol{k}}
\end{aligned}
$$

Which reduces to the scalar equation:

$$
{ }^{1} I_{1} \ddot{q}_{1}=L c_{1} f_{2 \rightarrow 1_{y}}-L s_{1} f_{2 \rightarrow 1_{x}}-\frac{L}{2} c_{1} m g-L c_{1} M g+n_{1}-\tau_{h}
$$


The unknown hip force components, $f_{2 \rightarrow 1_{x}}$ and $f_{2 \rightarrow 1_{y}}$, will be solved for by applying the Newton-Euler equations to the swing leg (leg 2).

Swing Leg (Leg 2)

The Newton-Euler equations for the swing leg are as follows:

$$
\begin{aligned}
& m a_{\text {mid }_{2}}=f_{1 \rightarrow 2}+d_{2}+g_{\text {leg }} \\
& { }^{\text {hip }} I_{2} \ddot{\boldsymbol{\alpha}}_{2}=\underbrace{\left({ }^{\text {hip }} \boldsymbol{p}_{\text {mid }_{2}} \times \boldsymbol{g}_{\text {leg }}\right)}_{\text {Leg weight moment }}+\underbrace{\boldsymbol{n}_{2}}_{\begin{array}{c}
\text { Drag } \\
\text { moment }
\end{array}}+\underbrace{\boldsymbol{\tau}_{h}}_{\begin{array}{c}
\text { Hip } \\
\text { torque }
\end{array}}
\end{aligned}
$$

Expanding the vectors in the torque equation and taking the cross product:

$$
{ }^{h i p} I_{2}\left[\begin{array}{c}
0 \\
0 \\
\ddot{q}_{2}
\end{array}\right]=\left(\left[\begin{array}{c}
0 \\
0 \\
-\frac{L}{2} c_{2} m g
\end{array}\right]\right)+n_{2} \widehat{\boldsymbol{k}}+\tau_{h} \widehat{\boldsymbol{k}}
$$

This reduces to the scalar equation:

$$
{ }^{h i p} I_{2} \ddot{q}_{2}=-\frac{L}{2} c_{2} m g+n_{2}+\tau_{h}
$$

Turning to Newton's equations for the swing leg and expanding the vectors: 


$$
m\left[\begin{array}{c}
-L \ddot{q}_{1} s_{1}-L \dot{q}_{1}^{2} c_{1}-\frac{L}{2} \ddot{q}_{2} s_{2}-\frac{L}{2} \dot{q}_{2}^{2} c_{2} \\
L \ddot{q}_{1} c_{1}-L \dot{q}_{1}^{2} s_{1}+\frac{L}{2} \ddot{q}_{2} c_{2}-\frac{L}{2} \dot{q}_{2}^{2} s_{2} \\
0
\end{array}\right]=-\left[\begin{array}{c}
f_{2 \rightarrow 1_{x}} \\
f_{2 \rightarrow 1_{y}} \\
0
\end{array}\right]+\left[\begin{array}{c}
-d_{2} s_{2} \\
d_{2} c_{2} \\
0
\end{array}\right]+\left[\begin{array}{c}
0 \\
-m g \\
0
\end{array}\right]
$$

This yields two scalar equations, which can be solved for the unknown components of the reaction force at the hip:

$$
\begin{aligned}
& m\left(-L \ddot{q}_{1} s_{1}-L \dot{q}_{1}^{2} c_{1}-\frac{L}{2} \ddot{q}_{2} s_{2}-\frac{L}{2} \dot{q}_{2}^{2} c_{2}\right)=-f_{2 \rightarrow 1}-d_{2} s_{2} \\
& m\left(L \ddot{q}_{1} c_{1}-L \dot{q}_{1}^{2} s_{1}+\frac{L}{2} \ddot{q}_{2} c_{2}-\frac{L}{2} \dot{q}_{2}^{2} s_{2}\right)=-f_{2 \rightarrow 1_{y}}+d_{2} c_{2}-m g \\
& f_{2 \rightarrow 1_{x}}=m\left(L \ddot{q}_{1} s_{1}+L \dot{q}_{1}^{2} c_{1}+\frac{L}{2} \ddot{q}_{2} s_{2}+\frac{L}{2} \dot{q}_{2}^{2} c_{2}\right)-d_{2} s_{2} \\
& f_{2 \rightarrow 1 y}=m\left(-L \ddot{q}_{1} c_{1}+L \dot{q}_{1}^{2} s_{1}-\frac{L}{2} \ddot{q}_{2} c_{2}+\frac{L}{2} \dot{q}_{2}^{2} s_{2}\right)+d_{2} c_{2}-m g
\end{aligned}
$$


These expressions can then be substituted back into the Euler equations for the stance leg:

$$
\begin{aligned}
& { }^{1} I_{1} \ddot{q}_{1}=L c_{1}\left[m\left(-L \ddot{q}_{1} c_{1}+L \dot{q}_{1}^{2} s_{1}-\frac{L}{2} \ddot{q}_{2} c_{2}+\frac{L}{2} \dot{q}_{2}^{2} s_{2}\right)+d_{2} c_{2}-m g\right] \\
& -L s_{1}\left[m\left(L \ddot{q}_{1} s_{1}+L \dot{q}_{1}^{2} c_{1}+\frac{L}{2} \ddot{q}_{2} s_{2}+\frac{L}{2} \dot{q}_{2}^{2} c_{2}\right)-d_{2} s_{2}\right]-\frac{L}{2} c_{1} m g-L c_{1} M g+ \\
& n_{1}-\tau_{h}
\end{aligned}
$$

Distributing $m, c_{1}$, and $s_{1}$ and dividing the equation by $L$ :

$$
\begin{aligned}
& \frac{{ }_{I_{1}}}{L} \ddot{q}_{1}=-m L \ddot{q}_{1} c_{1}^{2}+m L \dot{q}_{1}^{2} c_{1} s_{1}-m_{2}^{L} \ddot{q}_{2} c_{1} c_{2}+m \frac{L}{2} \dot{q}_{2}^{2} c_{1} s_{2}+d_{2} c_{1} c_{2}- \\
& c_{1} m g-m L \ddot{q}_{1} s_{1}^{2}-m L \dot{q}_{1}^{2} c_{1} s_{1}-m_{2}^{L} \ddot{q}_{2} s_{1} s_{2}-m_{2}^{L} \dot{q}_{2}^{2} s_{1} c_{2}+d_{2} s_{1} s_{2}-\frac{1}{2} c_{1} m g- \\
& c_{1} M g+\frac{n_{1}-\tau_{h}}{L}
\end{aligned}
$$

This equation can be simplified as follows. Grouping like terms:

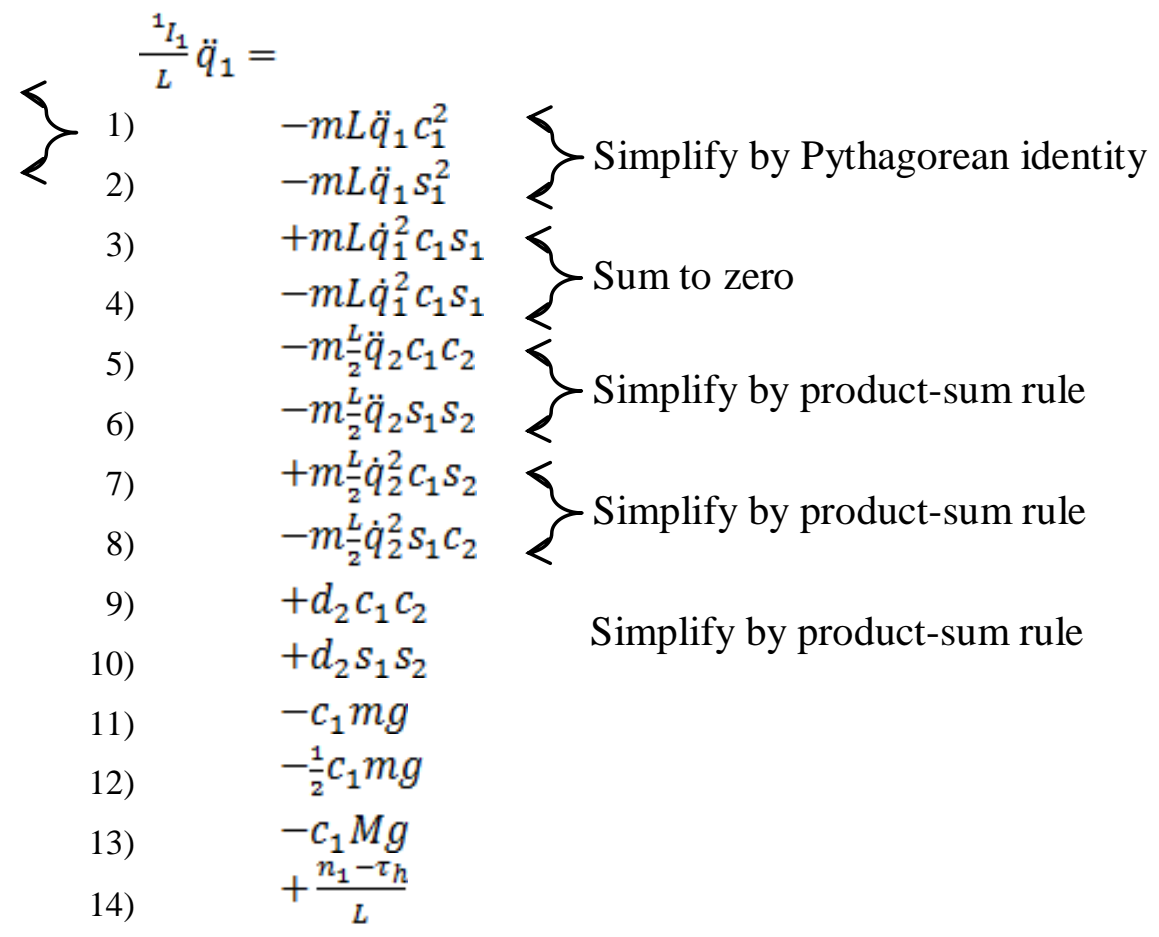


The equation thus becomes:

$$
\frac{{ }^{1} L_{1}}{L} \ddot{q}_{1}=-m L \ddot{q}_{1}-m_{2}^{L} \ddot{q}_{2} c_{1-2}-m_{2}^{L} \dot{q}_{2}^{2} s_{1-2}+d_{2} c_{1-2}-c_{1}\left(\frac{3}{2} m+M\right) g+\frac{n_{1}-\tau_{h}}{L}
$$

Grouping the angular acceleration terms:

$$
\left(\frac{{ }^{1} L_{1}}{L}+m L\right) \ddot{q}_{1}=\left(-m_{-2}^{L} c_{1-2}\right) \ddot{q}_{2}-m_{-2}^{L} \dot{q}_{2}^{2} s_{1-2}+d_{2} c_{1-2}-c_{1}\left(\frac{3}{2} m+M\right) g+\frac{n_{1}-\tau_{h}}{L}
$$

Substituting the expression for $\ddot{q}_{2}$ into the above equation and solving for $\ddot{q}_{1}$, the equations of motion are:

$$
\begin{aligned}
& \ddot{q}_{1}=\frac{-m \frac{L}{2} c_{1-2}\left(\frac{\frac{L}{2} c_{2} m g+n_{2}+\tau_{h}}{h i p}\right)-m_{2} \dot{q}_{2}^{2} s_{1-2}+d_{2} c_{1-2}-c_{1}\left(\frac{3}{2} m+M\right) g+\frac{n_{1}-\tau_{h}}{L}}{\left(\frac{{ }^{1} I_{1}}{L}+m L\right)} \\
& \ddot{q}_{2}=\frac{-\frac{L}{2} c_{2} m g+n_{2}+\tau_{h}}{h i p} I_{2}
\end{aligned}
$$


Converting to first order

This system of two second-order differential equations must be converted to a system of four first-order equations to allow for numerical propagation using the ode 45 function in MATLAB. Defining a state vector $\boldsymbol{q}$ :

$$
\boldsymbol{q}=\left[\begin{array}{l}
q_{1} \\
\dot{q}_{1} \\
q_{2} \\
\dot{q}_{2}
\end{array}\right]
$$

The equations of motion can be expressed by the first-order system

$$
\dot{\boldsymbol{q}}=\left[\begin{array}{l}
\dot{q}_{1} \\
\ddot{q}_{1}=f\left(q_{1}, q_{2}, \dot{q}_{2}\right) \\
\dot{q}_{2} \\
\ddot{q}_{2}=f\left(q_{1}, q_{2}, \dot{q}_{1}\right)
\end{array}\right],
$$

Where the expressions for $\ddot{q}_{1}$ and $\ddot{q}_{2}$ are taken from the second-order equations. The waker model is implemented in MATLAB in Appendices 5e and 5f. 
Drag force, moment, and hip torque

In order to solve the equations of motion, the drag force, drag moment, and hip torque terms must be defined:

\section{Hip torque:}

$$
\tau_{h}=k\left(\theta_{1}-\theta_{2}+\pi\right)
$$

Drag force and moment for the stance leg:

For the purpose of computing the drag on each leg, it is important to note that the walker is assumed to be on a treadmill which is moving backwards at the same average speed that the walker is moving forwards. Therefore, while the position of the stance foot is static relative to the walking surface, it is not, in fact, static relative to the surrounding medium. An assumption is made that the horizontal position of the hip is static relative to the water. While the hip in fact moves slightly faster than the treadmill in the middle of each step and slower at the beginning and end of the step, the assumption that it is static in the horizontal direction is a good one for small values of $\theta_{1}$.

Given the static hip assumption, the position and velocity of a point on the stance leg, at a distance $r$ from the hip, are described by the vectors 


$$
{ }^{\text {hip }} \boldsymbol{p}_{r}=\left[\begin{array}{c}
-r c_{1} \\
-r s_{1} \\
0
\end{array}\right] \quad \text { hip } v_{r}=\left[\begin{array}{c}
r \dot{q}_{1} s_{1} \\
-r \dot{q}_{1} c_{1} \\
0
\end{array}\right]
$$

The component of velocity relevant to determining drag force is the component perpendicular to the leg:

$$
\begin{aligned}
& v_{\perp, r}=r \dot{q}_{1} s_{1}^{2}+r \dot{q}_{1} c_{1}^{2} \\
& v_{\perp, r}=r \dot{q}_{1}
\end{aligned}
$$

Drag per unit length at station $r$ is estimated as:

$$
\frac{\partial d_{1}}{\partial r}=k_{d} v_{\perp, r}^{2}=k_{d} r^{2} \dot{q}_{1}^{2}
$$

where $k_{d}=\frac{1}{2} \rho w c_{d}, w$ is the average width of the leg, $\rho$ is the density of water, and $c_{d}$ is the drag coefficient of the leg. To obtain the magnitude of the drag force on the leg, $\frac{\partial d}{\partial r}$ is integrated along the length of the leg:

$$
\begin{aligned}
& d_{1}=\int_{r=0}^{L} \frac{\partial d_{1}}{\partial r}=k_{d} \dot{q}_{1}^{2} \int_{r=0}^{L} r^{2} \\
& d_{1}=\frac{1}{3} k_{d} \dot{q}_{1}^{2} L^{3}
\end{aligned}
$$


Similarly, to obtain the total moment induced by the drag force about the hip, $r \frac{\partial d}{\partial r}$ is integrated along the length of the leg:

$$
\begin{aligned}
& n_{1}=\int_{r=0}^{L} r \frac{\partial d_{1}}{\partial r}=k_{d} \dot{q}_{1}^{2} \int_{r=0}^{L} r^{3} \\
& n_{1}=\frac{1}{4} k_{d} \dot{q}_{1}^{2} L^{4}
\end{aligned}
$$

Drag force and moment for the swing leg:

Turning to the swing leg, the position and velocity of a point on the swing leg, at a distance $r$ from the hip, are described by the vectors

$$
{ }^{h i p} \boldsymbol{p}_{r}=\left[\begin{array}{c}
r c_{2} \\
r s_{2} \\
0
\end{array}\right] \quad{ }^{\text {hip }} \boldsymbol{v}_{r}=\left[\begin{array}{c}
-r \dot{q}_{2} s_{2} \\
r \dot{q}_{2} c_{2} \\
0
\end{array}\right]
$$

The component of velocity perpendicular to the leg is

$$
v_{\perp, r}=r \dot{q}_{2}
$$

By analogy with the drag on the stance leg:

$$
\begin{aligned}
& d_{2}=\frac{1}{3} k_{d} \dot{q}_{2}^{2} L^{3} \\
& n_{2}=\frac{1}{4} k_{d} \dot{q}_{2}^{2} L^{4}
\end{aligned}
$$


Because the drag force and moment equations depend on the square of the rates of rotation of each leg, care must be taken to ensure that the drag forces and moments have the correct sign during all parts of the stride. Based on the sign conventions adhered to thus far, the drag force and moment equations must be modified before they can be implemented in code:

$$
\begin{aligned}
& d_{1}=\frac{1}{3} k_{d} \dot{q}_{1}^{2} L^{3} * \operatorname{sign}\left(q_{1}\right) \\
& n_{1}=\frac{1}{4} k_{d} \dot{q}_{1}^{2} L^{4} * \operatorname{sign}\left(q_{1}\right) \\
& d_{2}=-\frac{1}{3} k_{d} \dot{q}_{2}^{2} L^{3} * \operatorname{sign}\left(q_{2}\right) \\
& n_{2}=-\frac{1}{4} k_{d} \dot{q}_{2}^{2} L^{4} * \operatorname{sign}\left(q_{2}\right)
\end{aligned}
$$

\section{Tuning the Passive Dynamic Walker}

Although a passive dynamic walker is a system that, by definition, will maintain a stable gait without external control, the characteristics of that gait require some degree of tuning in order to produce gait metrics that are comparable to those of a human subject. In addition, the presence of a drag force in the model necessitates additional corrective inputs to the walker in order to maintain stability.

In the case of the passive dynamic walker model described above, four parameters were tuned in order to generate stable gaits and to target two gait metrics - walking speed, and the non-dimensional velocity exponent $\beta$, which relates non- 
dimensionalized step length and velocity: $s \sim v^{\beta}$. ${ }^{[10]}$ As the purpose of the dynamic model was to replicate the gait characteristics of actual test subjects, the leg and torso mass and leg length constants in the model were set to match those of each subject. In addition, the target values of speed and $\beta$ were determined from the physical data, with the target speed being the average speed of the subject over a particular run, and the target $\beta$ calculated as an average over all subjects for each specific test case (i.e., each combination of simulated gravity level, treadmill power, and whether the subject was in water or on dry land). Although the biomechanics literature suggests that a value for $\beta$ of 0.42 is typical for adults ${ }^{[10]}$, the values calculated from the motioncapture data varied significantly across the test cases, and it was decided that using a case-specific value for $\beta$ to tune the walker would yield a more accurate model of the subject's gait than would relying on a textbook value intended to apply to a much narrower range of environmental conditions than those to which the subjects of this study were exposed.

Given target values for walking speed and the velocity exponent, the first gait parameter, the starting maximum angle of the legs from the vertical, was determined as follows. First, non-dimensionalizing the target speed using leg length and the acceleration of gravity:

$$
v=\frac{\text { speed }}{\sqrt{L g}}
$$


From the definition of $\beta$, the non-dimensional stride length is calculated, and the dimensional stride length determined:

$s=v^{\beta}$

steplength $=s * L$

From here, assuming a symmetrical stance in the sagittal plane, the angle of each leg from the vertical is calculated by geometry:

start_leg_angle $=\sin ^{-1}\left(\frac{\text { steplength }}{4}\right)$

The second gait parameter is $k$, the spring constant of the hip. By adjusting the value of $k$, the walker can be made to come arbitrarily close to the target speed, though this entails sacrificing some accuracy with regard to targeting $\beta$.

The third gait parameter is the magnitude of the initial toe-off from a spread-legged stance, expressed as the rate of change of $q_{1}$, the angle that the stance leg makes with the horizontal. The value of this initial toe-off is crucial for the first few steps, as an improperly-tuned walker will tend to either slip backwards or stumble forwards, falling over rather than attaining a stable gait. However, these asymmetries tend to damp out over a few gait cycles, provided they are below a critical threshold at the start. For the second and all subsequent toe-offs, the value of $d q_{1}$ immediately after toe-off is determined from the value of $d q_{2}$ immediately before heel-strike, such that the energy expended in heel-strike (modeled as a perfectly inelastic collision) is 
recovered exactly by the impulsive toe-off. Although the effect of the initial toe-off on gait is transitory, it is important to note that when computing average gait metrics over a simulated run, it is advisable to ignore the first few gait cycles. This is especially true for walkers experiencing perturbations such as drag, which the author found to significantly extend the time it took for the walker to achieve a stable gait.

For the non-zero drag case, it was determined through trial and error that impulsive toe-offs were insufficient to maintain a stable gait. Over time, with impulsive toe-off alone, step frequency increased as stride length decreased, eventually causing the walker to become unstable and stumble forward. This effect was countered with the introduction of an additional constant-valued hip torque, which acts to complement the inward torque of the hip torsional spring when the swing foot is behind the stance foot, and counteracts the effect of the hip spring when the swing foot moves in front of the stance foot. This imparts additional energy to the walker, and allows it to continue to take full strides despite the effects of drag.

This effect is intuitive for anyone who has tried to walk in water; on dry land, humans typically expend gait energy almost entirely at toe-off, using hip flexion/extension almost exclusively to tune the pendulum frequency of the leg, in the same manner as the conservative torsional spring does in the basic passive dynamic walker. ${ }^{[9]}$ However, when wading through water, this essentially-unpowered swinging action is not sufficient to get the swing foot to where it needs to be for the weight of the torso to come down on it - it becomes necessary to drive the swing leg forward at the hip. 
As an aside, it is interesting to note that the addition of ballast mass to the leg, especially at a significant distance from the hip, seems to counteract this effect. Although this study did not specifically address the effect of varying the moment of inertia of the leg on in-water gait, it was qualitatively observed that ballast on the legs allowed subjects to swing their legs through the water in a more natural gait motion than can be achieved unballasted. This is again an intuitive result - the increased angular inertia of the ballasted leg should allow it to maintain momentum through the fastest (and thus the most drag-inducing) part of the swing.

Returning to the tuning of the walker model, the walker was able to achieve a stable gait over a fairly wide range of values for the additional hip torque, although the presence of the additional parameter tended to result in a slight to moderate asymmetry between the first and second half of each step, as if the walker were continuously on the verge of stumbling.

Finally, one additional variable was introduced in tuning the walker model, although it represents not so much an additional gait parameter as a fudge factor. At high speeds in the zero-drag case, the walker demonstrated a tendency to over-rotate about the stance foot and eventually trip up. This problem was addressed by multiplying $d q_{1}$ at each toe-off by a constant factor in the range $(0.945,1.0)$. It's unclear what the cause of this problem is; however, the additional factor did not appear to affect the ability of the model to generate reasonable gait dynamics. 


\section{Chapter 10: Derivation of equations of motion for Symmetrical Impulsive Runner}

Constants

${ }^{h i p} I_{1} \quad$ Moment of inertia of the forward leg about the hip (used for the impulsive runner):

$$
{ }^{\text {hip }} I_{1}=\frac{m L^{2}}{3}
$$

\section{Equations of motion for the impulsive running gait}

Euler's equation for one leg, about the hip

$$
\begin{aligned}
& { }^{\text {hip }} I_{1} \boldsymbol{\alpha}_{1}=\left({ }^{\text {hip }} \boldsymbol{p}_{C H} \times \boldsymbol{g}_{\text {leg }}\right)+\boldsymbol{n}-\boldsymbol{\tau}_{h} \\
& { }^{h i p} I_{1}\left[\begin{array}{l}
0 \\
0 \\
\ddot{\theta}
\end{array}\right]=\left(\left[\begin{array}{c}
\frac{L}{2} s_{\theta} \\
-\frac{L}{2} c_{\theta} \\
0
\end{array}\right] \times\left[\begin{array}{c}
0 \\
-m g \\
0
\end{array}\right]\right)+n \widehat{\boldsymbol{k}}-\tau_{h} \widehat{\boldsymbol{k}} \\
& { }^{h i p} I_{1}\left[\begin{array}{l}
0 \\
0 \\
\ddot{\theta}
\end{array}\right]=\left[\begin{array}{c}
0 \\
0 \\
-m g_{\frac{L}{2} S_{\theta}}^{L}
\end{array}\right]+\left[\begin{array}{l}
0 \\
0 \\
n
\end{array}\right]+\left[\begin{array}{c}
0 \\
0 \\
-\tau_{h}
\end{array}\right] \\
& \ddot{\theta}=\frac{-m g_{\frac{2}{2} S_{\theta}+n-\tau_{h}}^{h i p}}{h i p} I_{1}
\end{aligned}
$$


Newton's equation for the whole system

$$
\begin{aligned}
& (M+2 m) \boldsymbol{a}_{C M}=\boldsymbol{g}_{\text {torso }}+2 \boldsymbol{g}_{\text {leg }}+\boldsymbol{d}_{1}+\boldsymbol{d}_{2} \\
& (M+2 m)\left[\begin{array}{l}
\ddot{x} \\
\ddot{y} \\
0
\end{array}\right]=\left[\begin{array}{c}
0 \\
-M g \\
0
\end{array}\right]+2\left[\begin{array}{c}
0 \\
-m g \\
0
\end{array}\right]+\left[\begin{array}{c}
-d c_{\theta} \\
-d s_{\theta} \\
0
\end{array}\right]+\left[\begin{array}{c}
d c_{\theta} \\
-d s_{\theta} \\
0
\end{array}\right] \\
& \ddot{x}_{C M}=0 \\
& \ddot{y}_{C M}=\frac{-(M+2 m) g-2 d s_{\theta}}{M+2 m}
\end{aligned}
$$

Where the subscript CM refers to the center of mass of the system, which is located a distance $\left(\frac{m L c_{\theta}}{M+2 m}\right)$ directly below the hip. Note that the system as a whole does not undergo any acceleration in the horizontal direction, due to the assumptions of symmetry made above.

\section{Converting to first order}

As with the walking case, the two second-order equations of motion describing the running case are converted into four first-order equations to allow for numerical integration in MATLAB. Defining a state vector $\boldsymbol{q}$ and its derivative: 


$$
\boldsymbol{q}=\left[\begin{array}{c}
q \\
\dot{q} \\
y \\
\dot{y}
\end{array}\right]
$$

$$
\dot{q}=\left[\begin{array}{l}
\dot{q} \\
\ddot{q}=f(q, \dot{q}, \dot{y}) \\
\dot{y} \\
\ddot{y}=f(q, \dot{q}, \dot{y})
\end{array}\right]
$$

Where the expressions for $\ddot{q}_{1}$ and $\ddot{q}_{2}$ are taken from the second-order equations. The impulsive runner model is implemented in MATLAB in Appendices $5 \mathrm{c}$ and $5 \mathrm{~d}$.

Drag force, moment, and hip torque

In order to solve the equations of motion, the drag force, drag moment, and hip torque terms must be defined:

Hip torque:

$$
\tau_{h}=2 k \theta
$$

Drag force and moment:

The position and velocity of a point on the leg, at a distance $r$ from the hip, are described by the vectors

$$
\boldsymbol{p}_{r}=\left[\begin{array}{c}
r s_{\theta} \\
y_{C M}+\frac{m L c_{\theta}}{M+2 m}-r c_{\theta} \\
0
\end{array}\right] \quad \boldsymbol{v}_{r}=\left[\begin{array}{c}
r \dot{\theta} c_{\theta} \\
\dot{y}_{C M}-\frac{m L \dot{\theta} s_{\theta}}{M+2 m}+r \dot{\theta} s_{\theta} \\
0
\end{array}\right]
$$


Taking the component of velocity perpendicular to the leg:

$$
v_{\perp, r}=\left(r \dot{\theta} c_{\theta}\right) c_{\theta}+\left(\dot{y}_{C M}+\frac{m L c_{\theta}}{M+2 m}-r c_{\theta}\right) s_{\theta}
$$

Drag per unit length at station $r$ is estimated as:

$$
\frac{\partial d}{\partial r}=k_{d} v_{\perp, r}^{2}=k_{d}\left[\left(r \dot{\theta} c_{\theta}\right) c_{\theta}+\left(\dot{y}_{C M}+\frac{m L c_{\theta}}{M+2 m}-r c_{\theta}\right) s_{\theta}\right]^{2}
$$

where $k_{d}=-\frac{1}{2} \rho w c_{d}, w$ is the average width of the leg, $\rho$ is the density of water, and $c_{d}$ is the drag coefficient of the leg. Squaring and grouping like terms:

$$
\frac{\partial d}{\partial r}=k_{d}\left[r \dot{\theta} c_{\theta}^{2}+y_{C M} s_{\theta}+c_{\theta} s_{\theta}\left(\frac{m L}{M+2 m}-r\right)\right]^{2}
$$

$$
\begin{aligned}
& \frac{\partial d}{\partial r}=k_{d}\left[r^{2} \dot{\theta}^{2} c_{\theta}^{4}+\dot{y}_{C M}^{2} s_{\theta}^{2}+\left(\frac{m L c_{\theta} s_{\theta}}{M+2 m}-r c_{\theta} s_{\theta}\right)^{2}+2 r \dot{\theta} c_{\theta}^{2} \dot{y}_{C M} s_{\theta}+\right. \\
& \left.2 r \dot{\theta} c_{\theta}^{3} s_{\theta}\left(\frac{m L}{M+2 m}-r\right)+2 y_{C M} s_{\theta}^{2} c_{\theta}\left(\frac{m L}{M+2 m}-r\right)\right]
\end{aligned}
$$

$$
\begin{aligned}
& \frac{\partial d}{\partial r}=k_{d}\left[r^{2}\left(\dot{\theta}^{2} c_{\theta}^{4}+c_{\theta}^{2} s_{\theta}^{2}-2 \dot{\theta} c_{\theta}^{3} s_{\theta}\right)+r\left(-2 \frac{m L c_{\theta}^{2} s_{\theta}^{2}}{M+2 m}+2 \dot{y}_{C M} \dot{\theta} c_{\theta}^{2} s_{\theta}+\right.\right. \\
& \left.\left.\frac{2 \dot{\theta} c_{\theta}^{3} s_{\theta} m L}{M+2 m}-2 \dot{y}_{C M} s_{\theta}^{2} c_{\theta}\right)+\left(\dot{y}_{C M}^{2} s_{\theta}^{2}+\left(\frac{m L c_{\theta} s_{\theta}}{M+2 m}\right)^{2}+\frac{2 \dot{y}_{C M} s_{\theta}^{2} c_{\theta} m L}{M+2 m}\right)\right]
\end{aligned}
$$


To obtain the magnitude of the drag force on the leg, $\frac{\partial d}{\partial r}$ is integrated along the length of the leg:

$$
\begin{aligned}
& d=\int_{r=0}^{L} \frac{\partial d}{\partial r}=k_{d}\left[\frac{1}{3} r^{3}\left(\dot{\theta}^{2} c_{\theta}^{4}+c_{\theta}^{2} s_{\theta}^{2}-2 \dot{\theta} c_{\theta}^{3} s_{\theta}\right)+\frac{1}{2} r^{2}\left(-2 \frac{m L c_{\theta}^{2} s_{\theta}^{2}}{M+2 m}+\right.\right. \\
& \left.2 \dot{y}_{C M} \dot{\theta} c_{\theta}^{2} s_{\theta}+\frac{2 \dot{\theta}_{\theta}^{3} s_{\theta} m L}{M+2 m}-2 \dot{y}_{C M} s_{\theta}^{2} c_{\theta}\right)+r\left(\dot{y}_{C M}^{2} s_{\theta}^{2}+\left(\frac{m L c_{\theta} s_{\theta}}{M+2 m}\right)^{2}+\right. \\
& \left.\left.\frac{2 \dot{y}_{C M} s_{\theta}^{2} c_{\theta} m L}{M+2 m}\right)\right]\left.\right|_{0} ^{L}
\end{aligned}
$$

$$
\begin{aligned}
& d=k_{d} {\left[\frac{1}{3} L^{3}\left(\dot{\theta}^{2} c_{\theta}^{4}+c_{\theta}^{2} s_{\theta}^{2}-2 \dot{\theta} c_{\theta}^{3} s_{\theta}\right)+\frac{1}{2} L^{2}\left(-2 \frac{m L c_{\theta}^{2} s_{\theta}^{2}}{M+2 m}+2 \dot{y}_{C M} \dot{\theta} c_{\theta}^{2} s_{\theta}+\frac{2 \dot{\theta} c_{\theta}^{3} s_{\theta} m L}{M+2 m}-\right.\right.} \\
& 2 y C M s \theta 2 c \theta+L y C M 2 s \theta 2+m L c \theta s \theta M+2 m 2+2 y C M s \theta 2 c \theta m L M+2 m
\end{aligned}
$$

Similarly, to obtain the total moment induced by the drag force about the hip, $r \frac{\partial d}{\partial r}$ is integrated along the length of the leg:

$$
\begin{aligned}
n=\int_{r=0}^{L} r \frac{\partial d}{\partial r}=k_{d}\left[\frac{1}{4} L^{4}\left(\dot{\theta}^{2} c_{\theta}^{4}+c_{\theta}^{2} s_{\theta}^{2}-2 \dot{\theta} c_{\theta}^{3} s_{\theta}\right)+\frac{1}{3} L^{3}\left(-2 \frac{m L c_{\theta}^{2} s_{\theta}^{2}}{M+2 m}+2 \dot{y}_{C M} \dot{\theta} c_{\theta}^{2} s_{\theta}+\right.\right. \\
\quad 2 \theta c \theta 3 s \theta m L M+2 m-2 y C M s \theta 2 c \theta+12 L 2 y C M 2 s \theta 2+m L c \theta s \theta M+2 m 2+2 y C M s \theta 2 c \theta m \\
\quad L M+2 m
\end{aligned}
$$




\section{Tuning the impulsive runner}

As with the walker model, the impulsive runner was adjusted to match the anthropometry of each subject and the parameters of each test case, and was tuned to generate desired gait metrics.

Three parameters were tuned; the hip spring constant $k$, the vertical toe-off velocity $d y_{0}$, and $d q_{0}$, the angular velocity of the forward leg just after toe-off. As noted above, the impulsive runner model is symmetric front to back, and so $d q_{0}$ is also the toe-off angular velocity of the rear leg. Contact with the ground occurs at cross-over, the point at which the forward and rear legs switch positions. Therefore, one of the targets in tuning the runner was what may be called return timing - the tendency to hit the ground just as the legs are crossing over.

The second quantitative target for tuning the runner was step length. At a given speed, the step length is simply the product of speed and step duration, which, for the impulsive runner, is simply the length of time the runner is off the ground. The final quantitative metric targeted was the maximum distance between the feet during the stride, which is fully determined from the angle and length of the legs.

Targeting step length resulted in a compromise in which another useful metric, the vertical displacement of the torso over the course of the stride, became less accurate (specifically, too large). This compromise is a direct result of the limitations on accuracy imposed by the assumption of impulsive, instantaneous ground contacts. In 
real gaits, there exists a finite walk-run transition regime ${ }^{[17]}$ that allows for alternating finite periods of single-support (as in walking) and no support (as in the majority of the time while running). This allows the runner to cover more ground per stride without the need for a large vertical leap.

The three tuned parameters and three targeted gait characteristics of the impulsive runner are highly coupled. Increasing the value of $d y_{0}$ tends to increase step length, while simultaneously increasing step duration and affecting return timing. Increasing the value of $d q_{0}$ tends to increase the maximum distance between the feet, and tends to decrease step length by increasing the downward component of drag on the legs. Increasing $k$ (tightening the hip spring) tends to reduce the maximum distance between the feet, and also affects return timing.

The full set of gait metric data for the walker and runner models can be found in Appendix 4. 


\section{Chapter 11: Analysis of the Extended Test Matrix}

Once gait metric data were obtained for the physical test cases and the dynamic models, these data could be analyzed in order to generate estimates for true lunar gravity gait metrics (goal 1), and to estimate the magnitude of the effect on each gait metric of gravity, drag, and modeling limitations (goal 2).

The four gait metrics of step length, vertical displacement of the torso, maximum angle between the legs, and pendulum frequency are each dependent on speed. Because speed varies between subjects, even for the same test case, and because certain model-derived datasets have fewer data points than the rest, the data points themselves cannot be directly added or subtracted to obtain estimates for goals 1 and 2. Instead, regression models are fit to each dataset (see Appendices 3 and 4), and these equations, being defined for all speeds, may be directly added and subtracted to produce the desired estimates. 


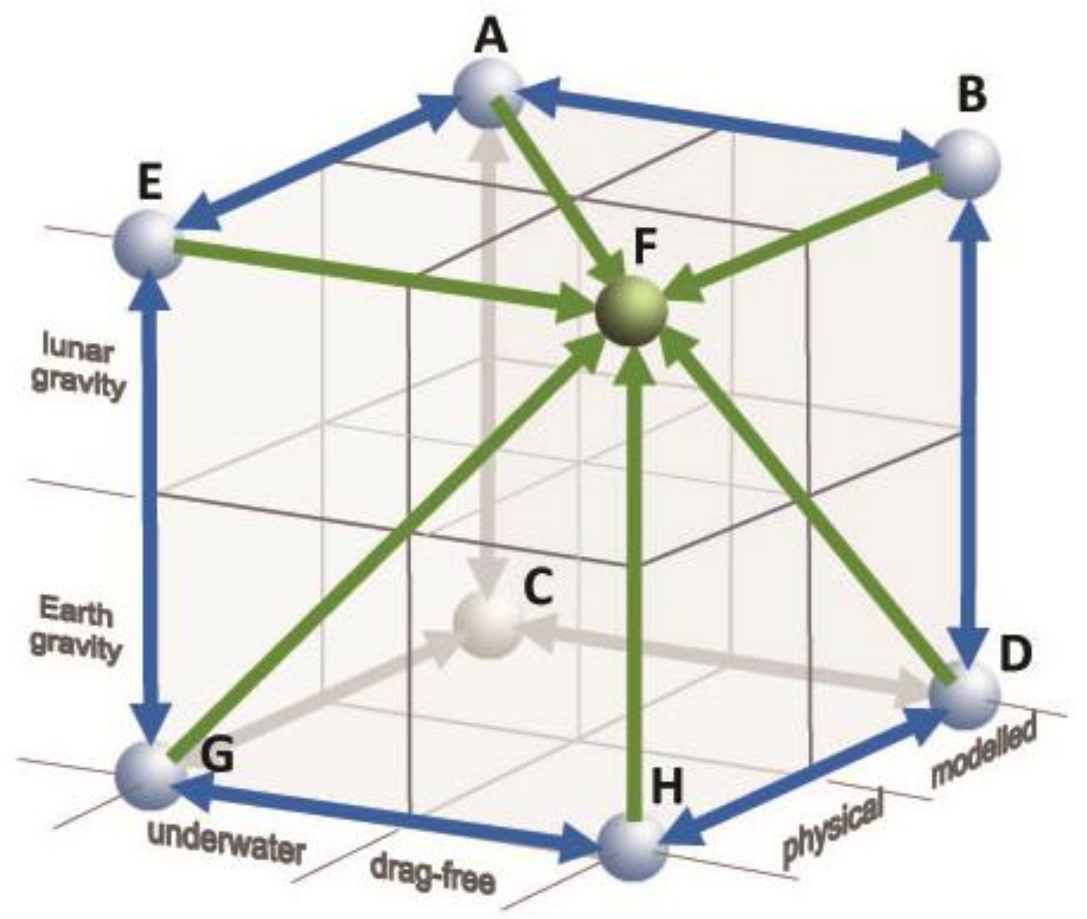

Figure 27: Visualization of the test matrix used to determine gait metric functions for true lunar gait

To address goal 1 (see Appendix 5g for MATLAB code, and analysis and conclusions section for plots), for each metric, three intermediate function estimates are generated, one for each face of the test matrix adjacent to the true lunar gravity environment. For example, given functions for some gait metric at vertex $E$, vertex $A$, and vertex $B$, the intermediate function for the metric at $F$ is estimated as: (function at $F)=($ function at $E)+($ function at $B)-($ function at $A)$. These intermediate function estimates are then combined in two different ways, to generate two new function estimates for the metric at $F$. The first combination is simply an un-weighted average of the three intermediate functions. The second combination is a weighted average, where weights 
are derived from the worst $\mathrm{R}^{2}$ value among the three terms in each intermediate function.

Turning to goal 2 (see Appendix 5h for MATLAB code, and analysis and conclusions section for plots), estimates for the magnitude of the effect of each dimension (gravity, drag, and model limitations) are computed by taking a weighted average of the estimates found by subtracting along opposite edges of the test matrix. For example, to estimate the magnitude of the effect of the difference in Earth and lunar gravity on pendulum frequency, the function for pendulum frequency at element $G$ is subtracted from that at element $E$, and the function at element $D$ is subtracted from that at element $B$. These two estimates are combined in a weighted average, with weights being derived from the worst $\mathrm{R}^{2}$ value in each estimate. In order to give meaning to the magnitide of the apparent effect of each dimension, each magnitude estimate is normalized by the second term of the difference.

\section{Goal 1: Estimating gait metrics for the true lunar gravity environment}

The following plots show estimates for each gait metric (step length, vertical displacement of the torso, maximum angle between the legs, and leg pendulum frequency) as a function of gait speed in the true lunar gravity environment.

Estimates of the gait metrics as a function of speed were obtained in the manner described above for each physically tested and modeled scenario. These estimates take the form of linear regression fits to the data for each test case. 
Because gait metrics were not directly obtained for true lunar gravity, but were instead obtained for the remaining nodes of the test matrix, estimates for gait metrics in true lunar gravity were obtained by a weighted average of three estimates based on the data obtained through physical testing and virtual modeling, as described in the project description and objectives section.

For example, to obtain an estimate of step length in true lunar gravity as a function of gait speed, three intermediate estimates were obtained as follows. The first estimate was obtained by summing the regression functions for step length at node E (underwater physical test in $1 / 6^{\text {th }} g$ ) and node $\mathrm{H}$ (drag-free physical test in $1 \mathrm{~g}$ ) and subtracting the regression function at node $\mathrm{G}$ (underwater physical test in $1 \mathrm{~g}$ ). This estimate is identified in the following plots at the "physical data estimate", so named because the three nodes involved are all on the same "face" of the test matrix, and represent data obtained during physical testing. Similar estimates are obtained for the "lunar gravity" face (nodes A, B, and E) and the "drag-free" face (nodes B, D, and $\mathrm{H})$.

The sums and differences are taken between the regression functions, rather than between the raw data points, because the data points between nodes do not correspond to one another; each data point corresponds to a unique gait speed, and the number of data points is not the same for all nodes (in particular, the impulsive runner 
model was not able to generate reasonable data at the lowest speeds, and these data points are thus absent).

A weight is assigned to each estimated gait metric function, corresponding to the worst (lowest) $\mathrm{R}^{2}$ value of the three regression functions combined to generate that estimate. A weighted average is then obtained across the three estimates, for each gait metric.

In addition to the weighted and un-weighted estimates, the following plots include the three intermediate estimates used to generate these averages.

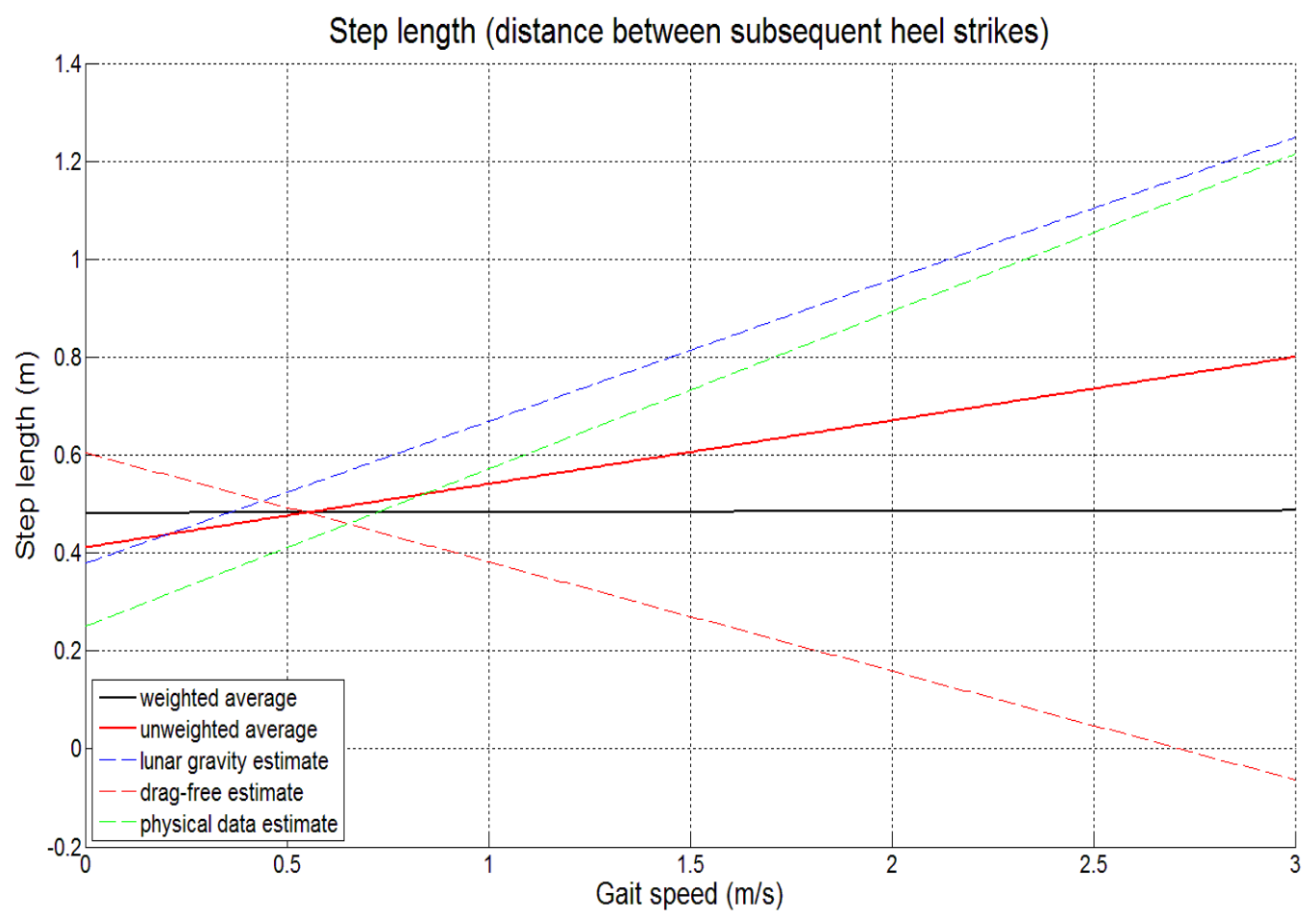

Figure 28: Step length vs. gait speed (extended test matrix) 
This weighted average indicates that step length is expected to be roughly constant with speed in true lunar gravity. The unweighted average indicates that step length is expected to increase with speed. Note that the lunar gravity and physical data estimates predict an increase in step length with gait speed (the expected result), while the drag-free estimate indicates the opposite trend. 


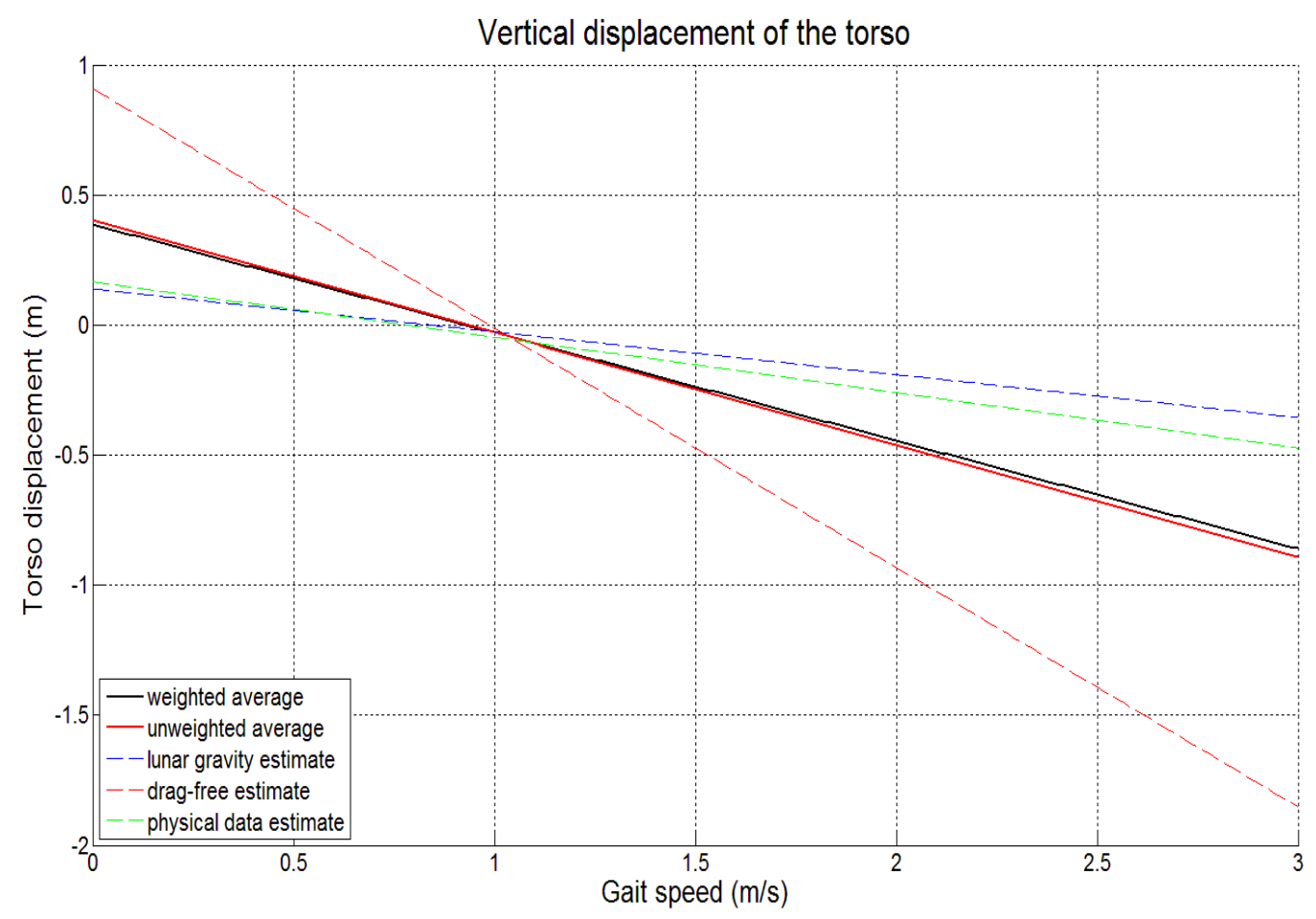

Figure 29: Vertical torso displacement vs. gait speed (extended test matrix)

This plot suggests that vertical displacement of the torso is expected to decrease with gait speed in true lunar gravity. Note the close agreement between the weighted and un-weighted averages. 


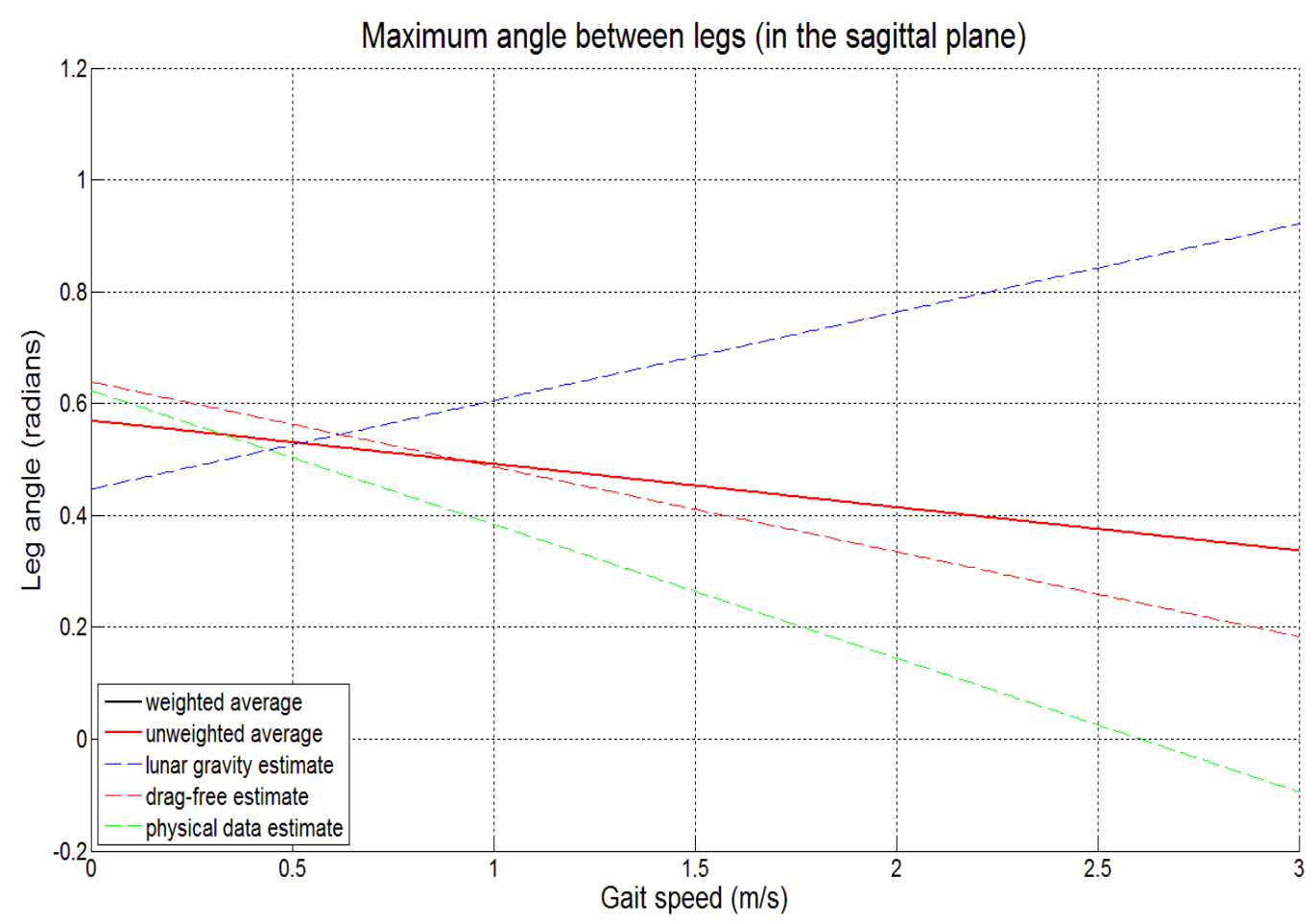

Figure 30: Maximum hip angle vs. gait speed (extended test matrix)

This plot suggests that the maximum angle between the legs is expected to decrease with gait speed in lunar gravity. The drag free and physical data estimates show such a trend, while the lunar gravity estimate indicates increasing maximum leg angle with gait speed. Note that the weighted and un-weighted averages overlap in this plot. 


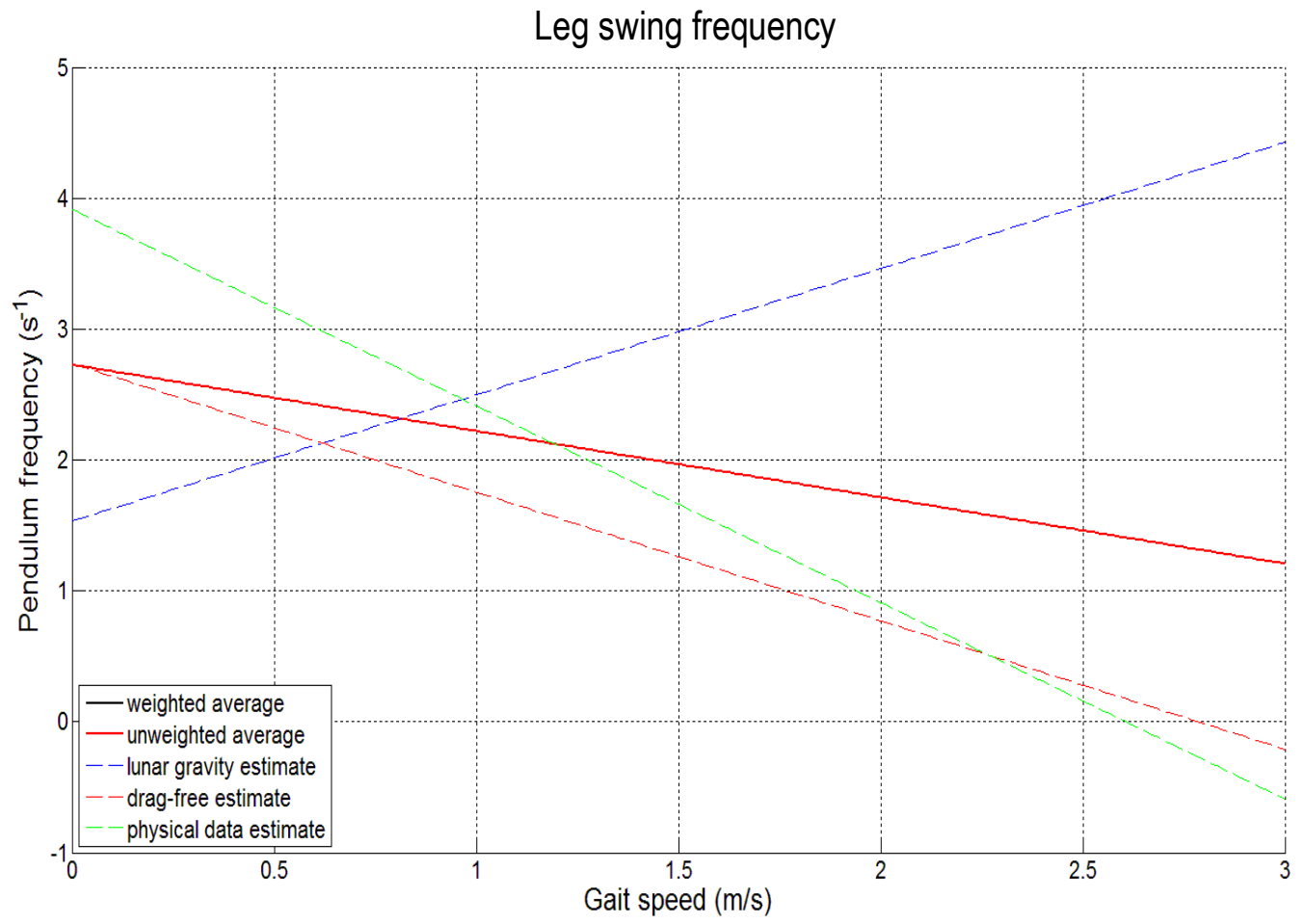

Figure 31: Leg swing frequency vs. gait speed (extended test matrix)

This plot indicates that leg swing frequency is expected to decrease as gait speed increases in true lunar gravity. The drag-free and physical data estimates show such a trend, while the lunar gravity estimate indicates an increasing pendulum frequency with gait speed. Note that the weighted and un-weighted averages overlap in this plot. 


\section{Goal 2: Estimating the impact of each test environment factor on each gait metric}

The following plots show the impact of each dimension of the test matrix - gravity level, presence of the underwater environment, and modeling limitations - on each of the four gait metrics considered - step length, vertical displacement of the torso, maximum angle between the legs, and leg pendulum frequency - as a function of walking/running speed.

Estimates of the gait metrics as a function of speed were obtained in the manner described above for each physically tested and modeled scenario. These estimates take the form of linear regression fits to the data for each test case.

In order to estimate the impact of each factor, the difference is taken between regression functions at adjacent nodes of the test matrix; for example, the impact of gravity level on step length may be assessed by taking the difference between the regression functions for step length at nodes B and D (drag-free models in $1 / 6^{\text {th }} g$ and $1 \mathrm{~g}$, respectively). A second estimate of the impact of gravity may be obtained from the two opposing nodes, $\mathrm{E}$ and $\mathrm{G}$, which represent the underwater, physical test data in $1 / 6^{\text {th }} g$ and $1 g$, respectively. In this manner, two estimates are obtained for the impact of each of the three factors on each gait metric function.

The differences are taken between the regression functions, rather than between the raw data points, because the data points between nodes do not correspond to one another; each data point corresponds to a unique gait speed, and the number of data 
points is not the same for all nodes (in particular, the impulsive runner model was not able to generate reasonable data at the lowest speeds, and these data points are thus absent).

A weight is assigned to each estimate, corresponding to the worse (lower) $\mathrm{R}^{2}$ value of the two regression functions whose difference comprises the estimate. For each of the three factors (gravity level, underwater environment, and modeling limitations) and for each gait metric, a weighted average is taken of the two estimates along the corresponding dimension of the test matrix. In this way, all available data can be used to estimate the impact of each factor on each metric, but with weaker relationships weighted less than stronger ones.

The full set of plots for the extended test matrix analyses are included in Appendices 2 and 3.

\section{Virtual modeling conclusions}

Based on the differences in gait metric functions between physically tested and simulated environments, one can assess the performance of the walking and running models in the different environments. The following plots show the difference in gait metric functions for two metrics: step frequency and vertical displacement of the torso, which were assessed for both the physical and simulated cases. Additional plots on the impact of each environmental factor - gravitational acceleration, water 
submersion, and modelling limitations - can be found in Appendix 3; the plots presented below are examples illustrating the impact of modelling limitations.

In each plot below, the blue dashed line is the difference between the regression fits for the underwater, lunar gravity case in the physical and simulated data. The green dashed line is the difference in regression fits for the dry land, $1 \mathrm{~g}$ case in the physical and simulated data. Each difference has been normalized by the magnitude of the gait metric function in the physical environment.

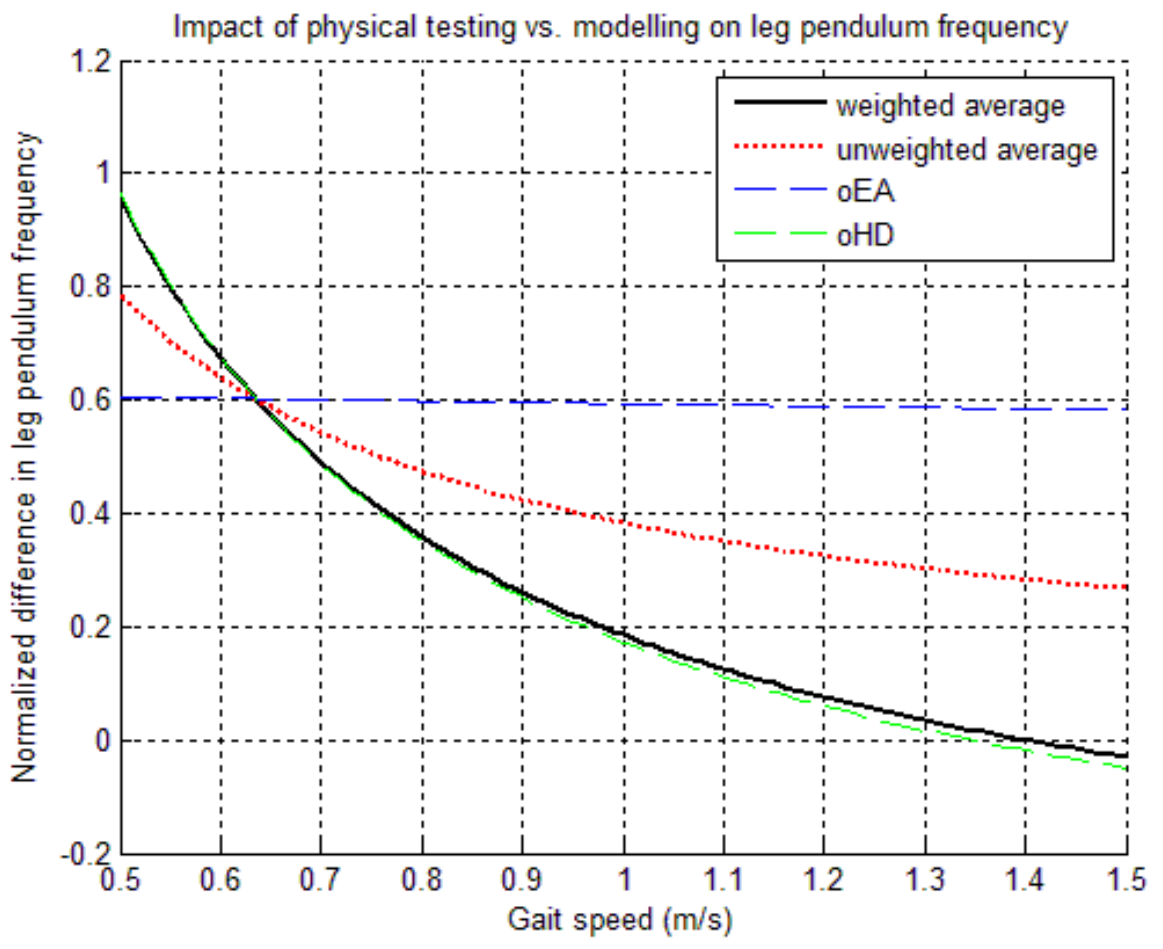

Figure 32: Modeling fidelity with regard to stride frequency

Considering the above plot, over the range of speeds from 0.5 to $1.5 \mathrm{~m} / \mathrm{s}$, the difference between modeled and physical data ranges from near $100 \%$ at lower 
speeds to near zero at higher speeds for the dry land, $1 \mathrm{~g}$ case. For the underwater, lunar gravity case, the difference between modeled and physical data is near $60 \%$ across the range of speeds. This indicates that, at most speeds, the dry, $1 \mathrm{~g}$ model was better able to match the corresponding physical data than the underwater, lunar gravity model, although both models tended to underestimate stride frequency.

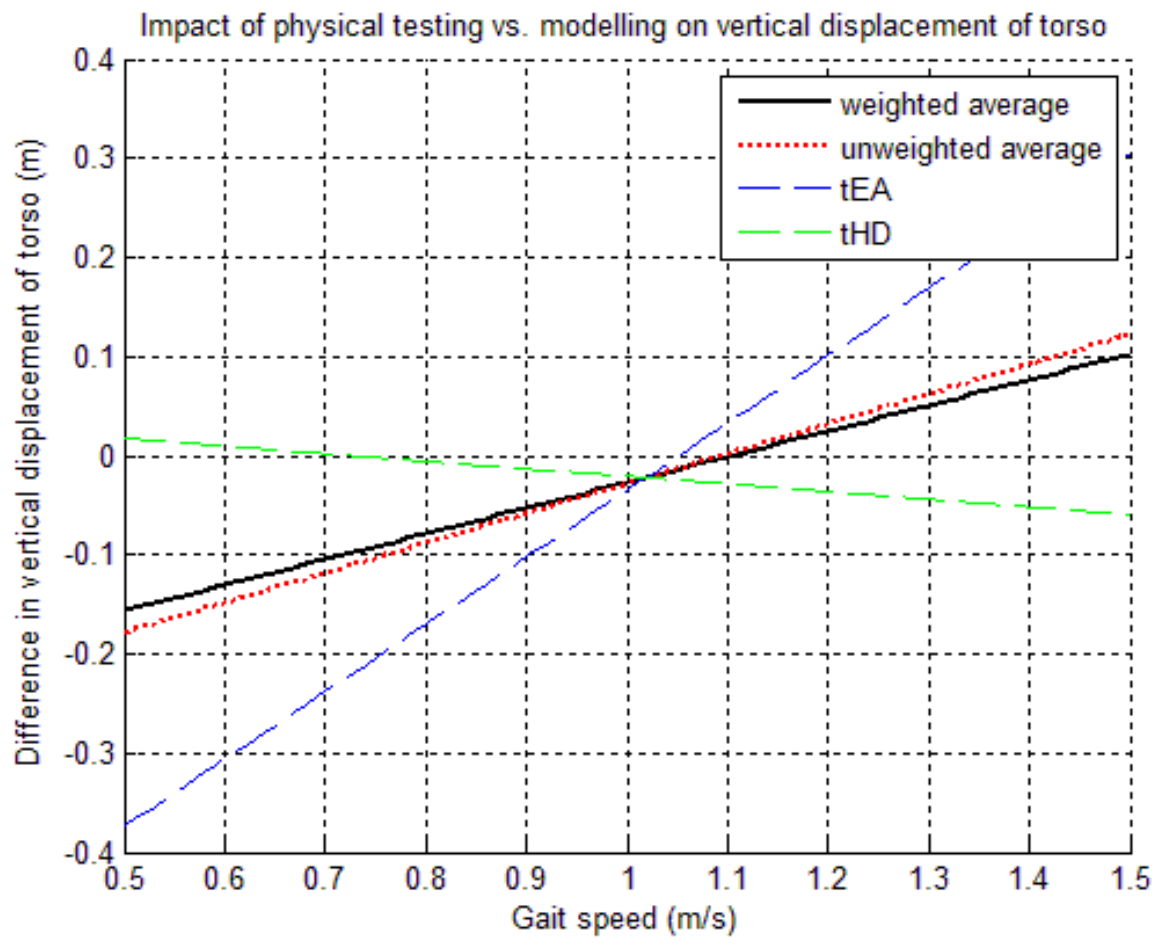

Figure 33: Modeling fidelity with regard to vertical hip displacement 
This plot shows that, at lower speeds, the underwater, $1 g$ model tended to overestimate torso displacement, while at higher speeds, it underestimated it. The dry, $1 \mathrm{~g}$ model more closely matches the corresponding physical data over the full range of speeds, differing by $0-10 \%$.

In general, the performance of the simulated gait models was too poor to justify reliance on these models for reasonable estimates of gait metrics. The two plots shown above illustrate the extent of the failure of the models to closely match the gait metric data obtained from motion-capture testing. In general, the underwater, lunar gravity model performed worse than the dry, $1 \mathrm{~g}$ model.

There are several likely reasons for the poor performance of the models. First, the assumption of pure impulsive running in the lunar gravity case is likely somewhat unrealistic, as the "duty cycle" - the portion of each stride in which one foot is in contact with the surface - was significant in the physical testing in the underwater, lunar gravity environment. Secondly, the drag terms incorporated into the equations of motion for each model were fairly simplistic, and the drag behavior of the human leg underwater was not experimentally verified.

Finally, the additional simplifications in both models likely resulted in model gait dynamics that did not closely match human gait dynamics. The lack of knees and ankles, the assumption of constant mass distribution along the length of the legs, and 
the two-dimensionality of the models may have skewed the resulting gait metric estimates.

While simple walker and runner models find great application in the theoretical study of gait types, transitions, and control mechanisms, ${ }^{[5][13][12]}$ it would seem, from the efforts undertaken in this study, that the complex and often non-linear behavior of human anatomy and physiology cannot adequately be captured by these simplified models for the purpose of predicting gait dynamics in untested environments. While more complex, more realistic models may better approach actual human gait dynamics, it is the author's opinion that simplified virtual gait models cannot take the place of physical testing in attempting to estimatate human gait dynamics in a given environment. 


\section{Chapter 12: Summary of Conclusions}

Underwater partial-gravity ballasting, treadmill gaits, and motion-capture dynamics analysis were used to assess gait dynamic metrics for subjects in three physical test environments: dry $1 g$, underwater $1 g$, and underwater lunar gravity. Gait metrics as a function of gait speed, including the metrics of step length, stride frequency, vertical displacement of the hip, and maximum angle of the hip, were compared on a nondimensionalized basis between the three physical test environments, by means of an analysis of covariance.

Based on the results of the analyses of covariance performed on th physical test data, it was concluded that differences in gravitational acceleration play a much more significant role in determining gait dynamics than the differences between dry and underwater environments. This result bodes well for the continued use of underwater partial-gravity ballasting with treadmill use as a means of simulating true reduced gravity environments, especially when alternate means of physical simulation, such as parabolic flight and suspension rigs, are unavailable or impractical.

As a secondary, supplemental study objective, virtual dynamics models were generated and used to try to re-create the gait dynamics observed in the physical testing. The resulting performance of the virtual models was extremely poor, especially for the underwater, lunar gravity case. It was concluded that the simplifications and physical assumptions included in the models made them unable to 
accurately estimate human gait dynamic metrics for the various environments. In the absence of an effective virtual model, it is believed that physical gait dynamics analysis must continue to play a role in the study of human gait in reduced gravity environments. 


\section{Appendices}

\section{Appendix 1: Physical test data}

Subject anthropometric measurements:

\begin{tabular}{|c|cccc|}
\hline $\begin{array}{c}\text { subject } \\
\#\end{array}$ & $\begin{array}{c}\text { knee height } \\
(\mathbf{m})\end{array}$ & $\begin{array}{c}\text { hip height } \\
(\mathbf{m})\end{array}$ & $\begin{array}{c}\text { hip-to-waist } \\
(\mathbf{m})\end{array}$ & $\begin{array}{c}\text { weight } \\
(\mathbf{k g})\end{array}$ \\
\hline $\mathbf{2}$ & 0.483 & 0.940 & 0.000 & 72.575 \\
$\mathbf{3}$ & 0.546 & 0.940 & 0.127 & 77.111 \\
$\mathbf{4}$ & 0.559 & 0.953 & 0.095 & 97.522 \\
$\mathbf{5}$ & 0.483 & 0.864 & 0.102 & 65.771 \\
$\mathbf{6}$ & 0.457 & 0.838 & 0.165 & 61.235 \\
mean & $\mathbf{0 . 5 0 6} \pm \mathbf{0 . 0 4}$ & $\mathbf{0 . 9 0 7} \pm \mathbf{0 . 0 5}$ & $\mathbf{0 . 0 9 8} \pm \mathbf{0 . 0 6}$ & $\mathbf{7 4 . 8 \pm 1 4 .}$ \\
\hline
\end{tabular}

Physical test data:

\begin{tabular}{|c|c|c|c|c|c|c|c|c|c|c|}
\hline \multirow[b]{2}{*}{ Gait metric } & \multirow[b]{2}{*}{ Subject number } & \multicolumn{3}{|c|}{$\begin{array}{c}1 g \\
\text { dry land }\end{array}$} & \multicolumn{3}{|c|}{$\begin{array}{c}1 \mathrm{~g} \\
\text { underwater }\end{array}$} & \multicolumn{3}{|c|}{$\begin{array}{c}\text { lunar g } \\
\text { underwater }\end{array}$} \\
\hline & & $15 \mathrm{~V}$ & $20 \mathrm{~V}$ & $25 \mathrm{~V}$ & $15 \mathrm{~V}$ & $20 \mathrm{~V}$ & $25 \mathrm{~V}$ & $15 \mathrm{~V}$ & $20 \mathrm{~V}$ & $25 \mathrm{~V}$ \\
\hline \multirow{7}{*}{$\underset{0}{\stackrel{5}{0}}$} & 2 & 0.368 & 0.333 & 0.318 & 0.313 & 0.276 & 0.289 & 0.550 & 0.492 & 0.476 \\
\hline & 3 & 0.410 & 0.369 & 0.355 & 0.286 & 0.310 & 0.313 & 0.479 & 0.464 & 0.540 \\
\hline & 4 & 0.446 & 0.389 & 0.363 & 0.273 & 0.242 & 0.353 & 0.587 & 0.611 & 0.675 \\
\hline & 5 & 0.453 & 0.448 & 0.425 & 0.244 & 0.209 & 0.189 & 0.608 & 0.656 & 0.777 \\
\hline & 6 & 0.465 & 0.479 & 0.398 & 0.337 & 0.291 & 0.312 & 0.726 & 0.832 & 0.972 \\
\hline & mean & 0.428 & 0.404 & 0.372 & 0.291 & 0.266 & 0.291 & 0.590 & 0.611 & 0.688 \\
\hline & stdev & 0.039 & 0.059 & 0.041 & 0.036 & 0.040 & 0.062 & 0.091 & 0.147 & 0.197 \\
\hline \multirow{8}{*}{$\underset{0}{\#} \underset{0}{0}$} & 2 & 0.038 & 0.079 & 0.135 & 0.034 & 0.070 & 0.092 & 0.043 & 0.086 & 0.135 \\
\hline & 3 & 0.038 & 0.082 & 0.139 & 0.033 & 0.060 & 0.080 & 0.098 & 0.163 & 0.186 \\
\hline & 4 & 0.034 & 0.077 & 0.126 & 0.033 & 0.060 & 0.072 & 0.099 & 0.138 & 0.264 \\
\hline & 5 & 0.042 & 0.088 & 0.149 & 0.037 & 0.078 & 0.114 & 0.126 & 0.262 & 0.400 \\
\hline & 6 & 0.044 & 0.094 & 0.156 & 0.035 & 0.069 & 0.087 & 0.184 & 0.220 & 0.290 \\
\hline & mean & 0.039 & 0.084 & 0.141 & 0.035 & 0.067 & 0.089 & 0.110 & 0.174 & 0.255 \\
\hline & stdev & 0.004 & 0.007 & 0.012 & 0.002 & 0.007 & 0.016 & 0.051 & 0.069 & 0.102 \\
\hline & stdev/mean & 0.105 & 0.083 & 0.083 & 0.052 & 0.111 & 0.177 & 0.463 & 0.396 & 0.399 \\
\hline \multirow{5}{*}{ 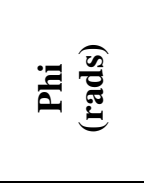 } & 2 & 0.617 & 0.565 & 0.712 & 0.592 & 0.822 & 0.788 & 0.434 & 0.516 & 0.725 \\
\hline & 3 & 0.545 & 0.648 & 0.787 & 0.583 & 0.534 & 0.663 & 0.481 & 0.673 & 0.561 \\
\hline & 4 & 0.441 & 0.591 & 0.671 & 0.646 & 0.709 & 0.602 & 0.623 & 0.509 & 0.554 \\
\hline & 5 & 0.586 & 0.788 & 0.744 & 0.552 & 0.808 & 0.984 & 0.499 & 0.620 & 0.560 \\
\hline & 6 & 0.370 & 0.467 & 0.578 & 0.489 & 0.550 & 0.747 & 0.333 & 0.397 & 0.466 \\
\hline \multirow{5}{*}{ 产 } & 2 & 0.872 & 0.722 & 0.613 & 0.987 & 0.813 & 0.723 & 1.538 & 1.415 & 1.282 \\
\hline & 3 & 0.814 & 0.681 & 0.585 & 1.047 & 0.817 & 0.739 & 1.388 & 1.233 & 1.116 \\
\hline & 4 & 0.797 & 0.683 & 0.602 & 1.077 & 0.905 & 0.729 & 1.229 & 1.121 & 0.947 \\
\hline & 5 & 0.703 & 0.579 & 0.512 & 1.027 & 0.813 & 0.715 & 1.089 & 0.914 & 0.804 \\
\hline & 6 & 0.676 & 0.543 & 0.512 & 0.885 & 0.755 & 0.622 & 0.904 & 0.814 & 0.730 \\
\hline \multirow{5}{*}{ 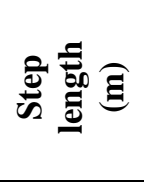 } & 2 & 0.514 & 0.616 & 0.683 & 0.555 & 0.651 & 0.666 & 0.397 & 0.514 & 0.583 \\
\hline & 3 & 0.480 & 0.593 & 0.662 & 0.577 & 0.608 & 0.633 & 0.540 & 0.618 & 0.597 \\
\hline & 4 & 0.446 & 0.576 & 0.653 & 0.597 & 0.676 & 0.597 & 0.482 & 0.519 & 0.606 \\
\hline & 5 & 0.420 & 0.499 & 0.574 & 0.576 & 0.658 & 0.700 & 0.458 & 0.554 & 0.602 \\
\hline & 6 & 0.406 & 0.477 & 0.580 & 0.478 & 0.569 & 0.574 & 0.454 & 0.447 & 0.461 \\
\hline \multirow{5}{*}{ 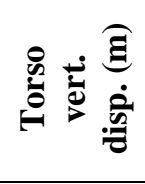 } & 2 & 0.025 & 0.032 & 0.042 & 0.028 & 0.049 & 0.049 & 0.130 & 0.113 & 0.105 \\
\hline & 3 & 0.021 & 0.031 & 0.043 & 0.025 & 0.047 & 0.059 & 0.090 & 0.089 & 0.072 \\
\hline & 4 & 0.019 & 0.029 & 0.037 & 0.038 & 0.057 & 0.056 & 0.065 & 0.078 & 0.058 \\
\hline & 5 & 0.028 & 0.036 & 0.042 & 0.029 & 0.045 & 0.052 & 0.059 & 0.048 & 0.042 \\
\hline & 6 & 0.014 & 0.021 & 0.029 & 0.022 & 0.029 & 0.024 & 0.022 & 0.027 & 0.028 \\
\hline
\end{tabular}


Physical test data (continued):

\begin{tabular}{|c|c|c|c|c|c|c|c|c|c|c|}
\hline \multirow[b]{2}{*}{ Gait metric } & \multirow[b]{2}{*}{ Subject number } & \multicolumn{3}{|c|}{$\begin{array}{c}1 \mathrm{~g} \\
\text { dry land }\end{array}$} & \multicolumn{3}{|c|}{$\begin{array}{c}1 \mathrm{~g} \\
\text { underwater }\end{array}$} & \multicolumn{3}{|c|}{$\begin{array}{c}\text { lunarg } \\
\text { underwater }\end{array}$} \\
\hline & & $15 \mathrm{~V}$ & $20 \mathrm{~V}$ & $25 \mathrm{~V}$ & $15 \mathrm{~V}$ & $20 \mathrm{~V}$ & $25 \mathrm{~V}$ & $15 \mathrm{~V}$ & $20 \mathrm{~V}$ & $25 \mathrm{~V}$ \\
\hline \multirow{7}{*}{$\underset{\mathfrak{E}}{\stackrel{\Xi}{\Xi}}$} & 2 & 0.017 & 0.000 & 0.000 & 0.300 & 0.347 & 0.660 & 1.000 & 1.000 & 1.000 \\
\hline & 3 & 0.000 & 0.000 & 0.000 & 0.000 & 0.492 & 1.000 & 1.000 & 1.000 & 1.000 \\
\hline & 4 & 0.000 & 0.000 & 0.000 & 0.000 & 0.959 & 0.960 & 1.000 & 1.000 & 1.000 \\
\hline & 5 & 0.000 & 0.000 & 0.000 & 0.000 & 0.197 & 0.750 & 1.000 & 1.000 & 1.000 \\
\hline & 6 & 0.000 & 0.000 & 0.000 & 0.035 & 0.469 & 0.920 & 0.940 & 0.964 & 1.000 \\
\hline & mean & 0.003 & 0.000 & 0.000 & 0.067 & 0.493 & 0.858 & 0.988 & 0.993 & 1.000 \\
\hline & stdev & 0.008 & 0.000 & 0.000 & 0.131 & 0.286 & 0.146 & 0.027 & 0.016 & 0.000 \\
\hline \multirow{8}{*}{ 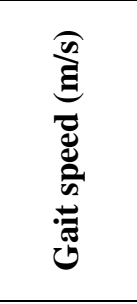 } & 2 & 0.590 & 0.853 & 1.114 & 0.563 & 0.801 & 0.921 & 0.258 & 0.364 & 0.455 \\
\hline & 3 & 0.590 & 0.871 & 1.131 & 0.551 & 0.744 & 0.857 & 0.389 & 0.501 & 0.535 \\
\hline & 4 & 0.559 & 0.844 & 1.085 & 0.554 & 0.747 & 0.819 & 0.393 & 0.463 & 0.640 \\
\hline & 5 & 0.598 & 0.861 & 1.121 & 0.560 & 0.810 & 0.979 & 0.420 & 0.607 & 0.750 \\
\hline & 6 & 0.601 & 0.879 & 1.133 & 0.540 & 0.754 & 0.849 & 0.502 & 0.550 & 0.631 \\
\hline & mean & 0.588 & 0.862 & 1.117 & 0.554 & 0.771 & 0.885 & 0.392 & 0.497 & 0.602 \\
\hline & stdev & 0.017 & 0.014 & 0.019 & 0.009 & 0.031 & 0.064 & 0.088 & 0.092 & 0.112 \\
\hline & stdev/mean & 0.028 & 0.016 & 0.017 & 0.016 & 0.041 & 0.073 & 0.224 & 0.185 & 0.186 \\
\hline \multirow{5}{*}{ 之章 } & 2 & 0.194 & 0.281 & 0.367 & 0.185 & 0.264 & 0.304 & 0.208 & 0.293 & 0.367 \\
\hline & 3 & 0.195 & 0.287 & 0.373 & 0.182 & 0.245 & 0.282 & 0.314 & 0.404 & 0.432 \\
\hline & 4 & 0.183 & 0.277 & 0.356 & 0.182 & 0.245 & 0.268 & 0.315 & 0.371 & 0.514 \\
\hline & 5 & 0.206 & 0.297 & 0.386 & 0.193 & 0.279 & 0.337 & 0.355 & 0.512 & 0.633 \\
\hline & 6 & 0.210 & 0.306 & 0.395 & 0.188 & 0.263 & 0.296 & 0.429 & 0.469 & 0.539 \\
\hline \multirow{5}{*}{ 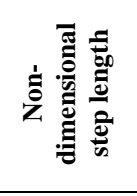 } & 2 & 0.547 & 0.655 & 0.727 & 0.591 & 0.692 & 0.708 & 0.422 & 0.547 & 0.621 \\
\hline & 3 & 0.511 & 0.631 & 0.704 & 0.614 & 0.647 & 0.673 & 0.574 & 0.657 & 0.635 \\
\hline & 4 & 0.469 & 0.607 & 0.687 & 0.628 & 0.711 & 0.628 & 0.508 & 0.546 & 0.638 \\
\hline & 5 & 0.489 & 0.580 & 0.667 & 0.669 & 0.765 & 0.814 & 0.532 & 0.644 & 0.700 \\
\hline & 6 & 0.483 & 0.568 & 0.691 & 0.569 & 0.678 & 0.684 & 0.541 & 0.533 & 0.548 \\
\hline \multirow{5}{*}{ 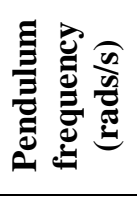 } & 2 & 0.573 & 0.692 & 0.815 & 0.507 & 0.615 & 0.692 & 0.325 & 0.354 & 0.390 \\
\hline & 3 & 0.615 & 0.734 & 0.854 & 0.478 & 0.612 & 0.677 & 0.360 & 0.405 & 0.448 \\
\hline & 4 & 0.628 & 0.733 & 0.831 & 0.464 & 0.552 & 0.686 & 0.407 & 0.446 & 0.528 \\
\hline & 5 & 0.712 & 0.863 & 0.977 & 0.487 & 0.615 & 0.699 & 0.459 & 0.547 & 0.622 \\
\hline & 6 & 0.740 & 0.922 & 0.976 & 0.565 & 0.662 & 0.679 & 0.553 & 0.614 & 0.685 \\
\hline \multirow{8}{*}{ 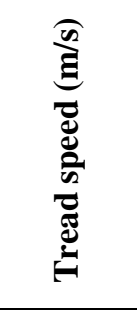 } & 2 & 0.589 & 0.868 & 1.145 & 0.575 & 0.835 & 1.078 & 0.622 & 0.907 & 1.089 \\
\hline & 3 & 0.582 & 0.866 & 1.128 & 0.549 & 0.767 & 1.039 & 0.633 & 0.906 & 1.265 \\
\hline & 4 & 0.548 & 0.834 & 1.089 & 0.538 & 0.756 & 0.941 & 0.503 & 0.764 & 1.033 \\
\hline & 5 & 0.588 & 0.854 & 1.124 & 0.563 & 0.804 & 1.115 & 0.652 & 0.888 & 1.162 \\
\hline & 6 & 0.601 & 0.884 & 1.148 & 0.589 & 0.865 & 0.834 & 0.581 & 0.853 & 1.120 \\
\hline & mean & 0.581 & 0.861 & 1.127 & 0.563 & 0.805 & 1.001 & 0.598 & 0.864 & 1.134 \\
\hline & stdev & 0.020 & 0.019 & 0.024 & 0.020 & 0.046 & 0.114 & 0.059 & 0.060 & 0.087 \\
\hline & stdev/mean & 0.034 & 0.022 & 0.021 & 0.036 & 0.057 & 0.113 & 0.099 & 0.069 & 0.077 \\
\hline \multirow{5}{*}{ 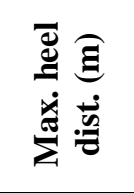 } & 2 & 0.642 & 0.727 & 0.775 & 0.615 & 0.693 & 0.737 & 0.496 & 0.568 & 0.622 \\
\hline & 3 & 0.632 & 0.727 & 0.801 & 0.677 & 0.721 & 0.688 & 0.636 & 0.701 & 0.635 \\
\hline & 4 & 0.562 & 0.670 & 0.733 & 0.671 & 0.721 & 0.642 & 0.509 & 0.541 & 0.635 \\
\hline & 5 & 0.571 & 0.649 & 0.710 & 0.695 & 0.813 & 0.825 & 0.536 & 0.603 & 0.642 \\
\hline & 6 & 0.525 & 0.587 & 0.685 & 0.536 & 0.610 & 0.587 & 0.480 & 0.484 & 0.504 \\
\hline
\end{tabular}




\section{Appendix 2: Extended test matrix - linear fit plots}

The following plots present linear regression fits for various gait metrics as a function of gait speed, with each plot illustrating the fits for two test environments which are adjacent in the extended test matrix; i.e., one environmental factor is different while the other two are the same. The three comparisons are: underwater vs. drag free, $1 \mathrm{~g}$ vs. lunar gravity, and physical vs. modeled. The regression fits from these plots are used in Appendix 3 to estimate the impact of each environmental factor on each gait metric, and to estimate gait metric curves for the true lunar gravity environment.

Step length vs. gait speed

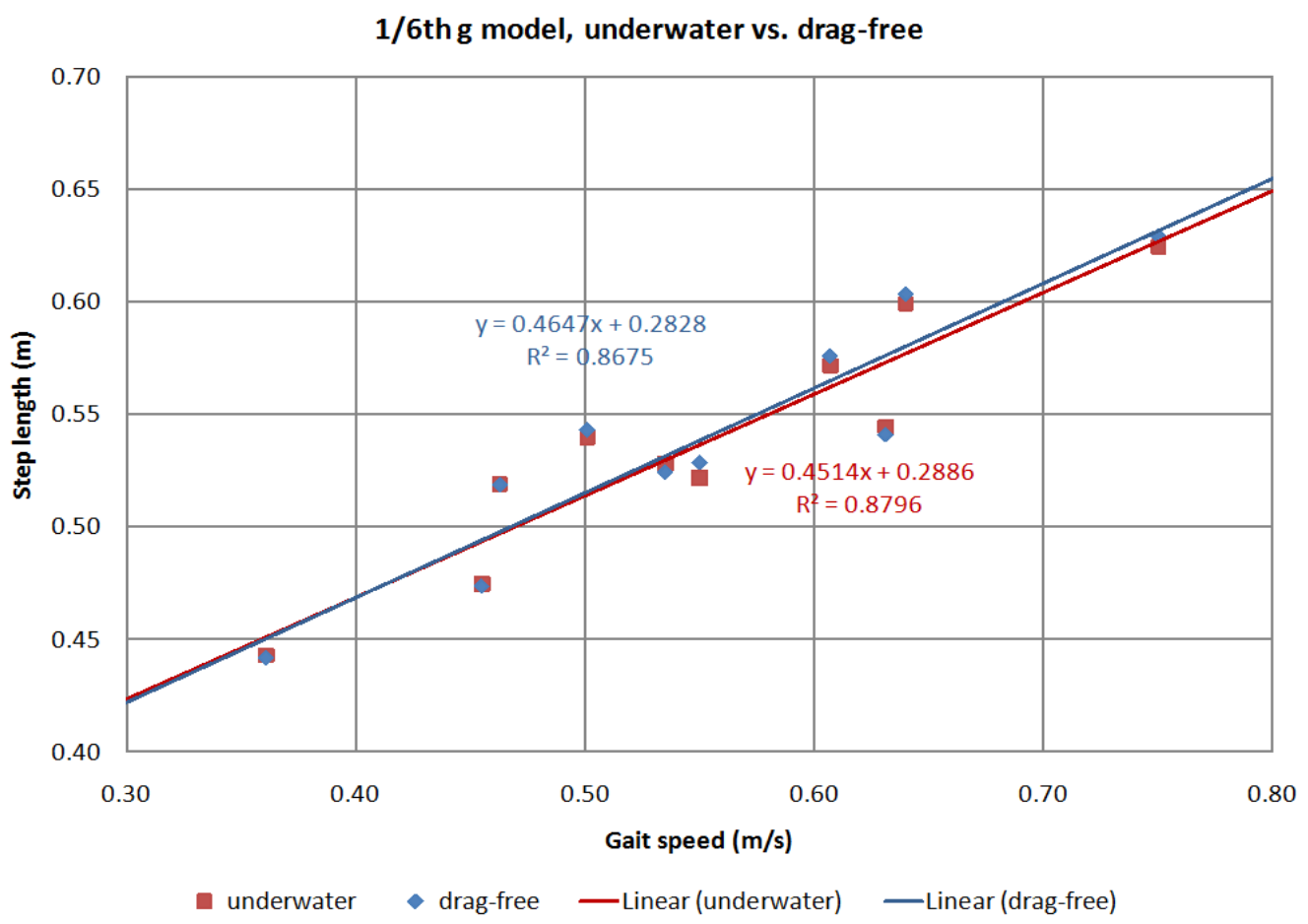


1/6th g underwater, model vs. physical

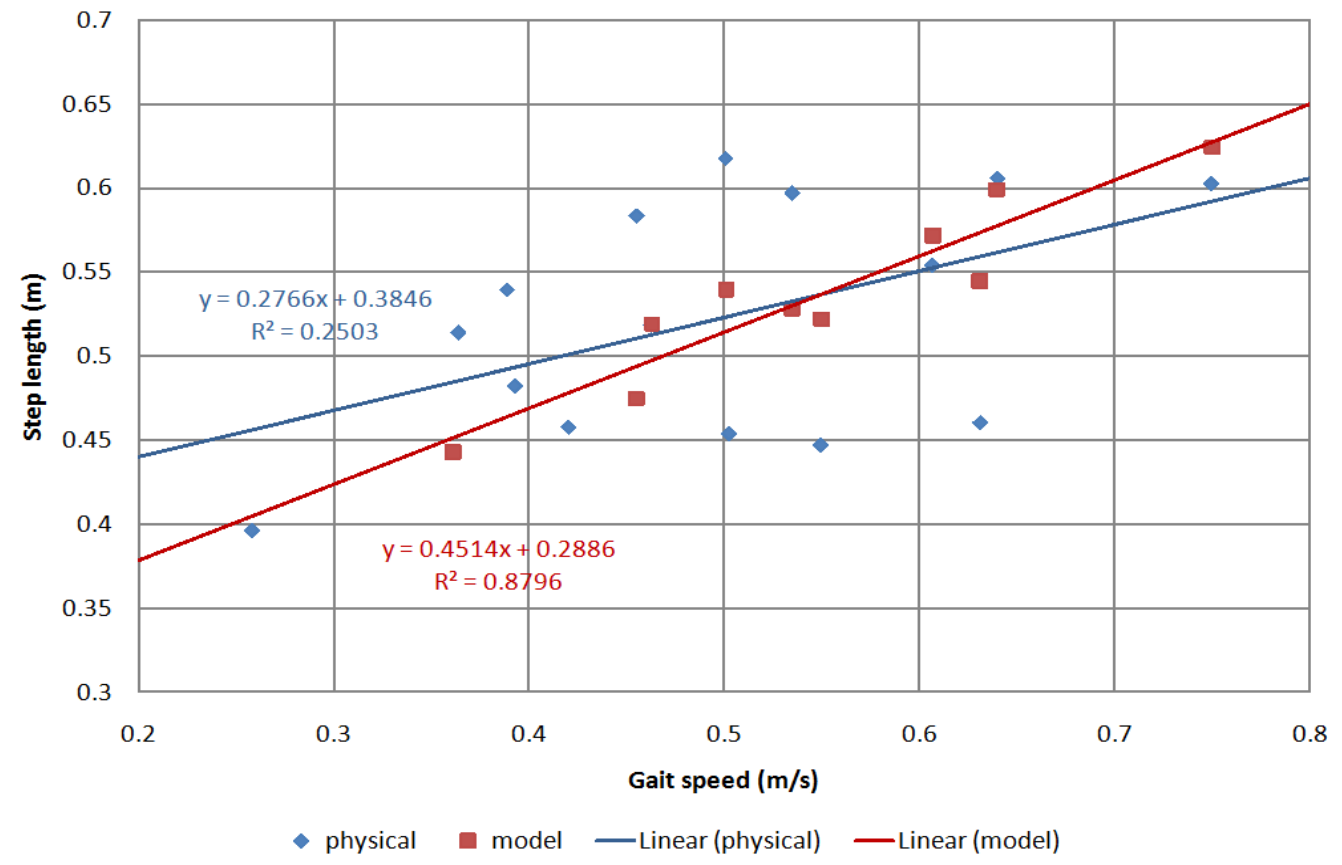

Drag-free model, $1 \mathrm{~g}$ vs. 1/6th g

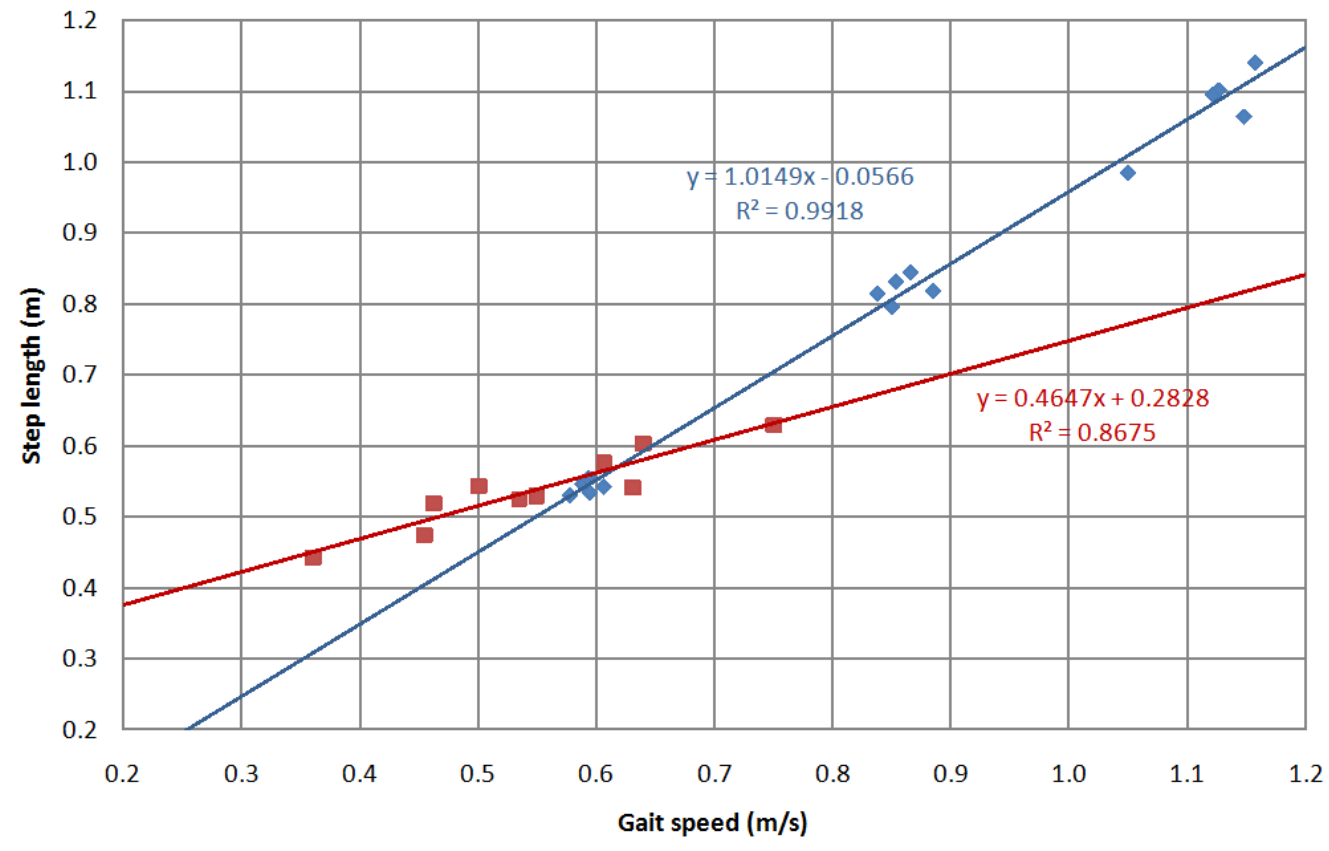

- $1 \mathrm{~g}$ 1/6th $\mathrm{g}$ - Linear $(1 \mathrm{~g})$ - Linear (1/6th g) 
Drag-free $1 \mathrm{~g}$, model vs. physical

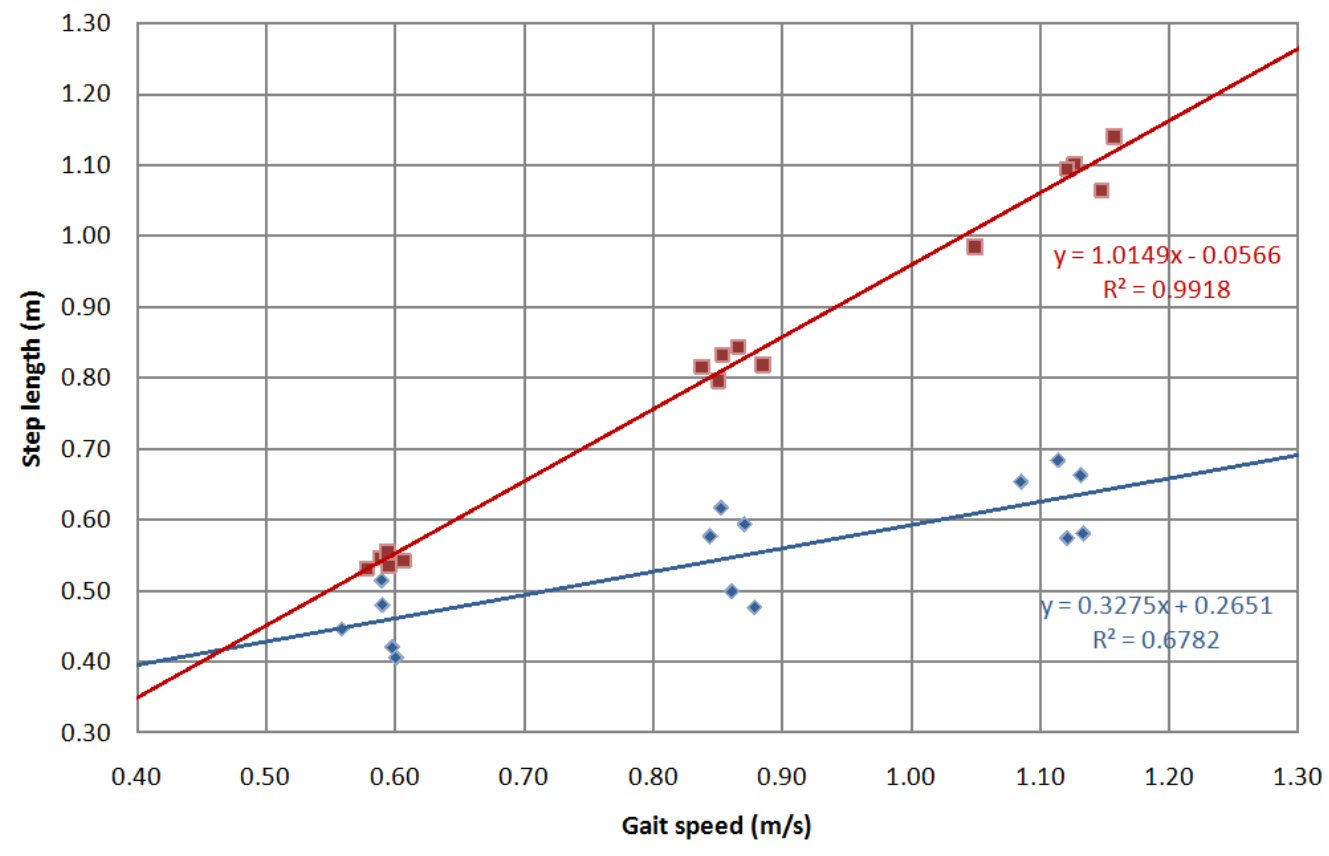

- physical m model —Linear (physical) —Linear (model)

Underwater physical, 1 g vs. 1/6th g

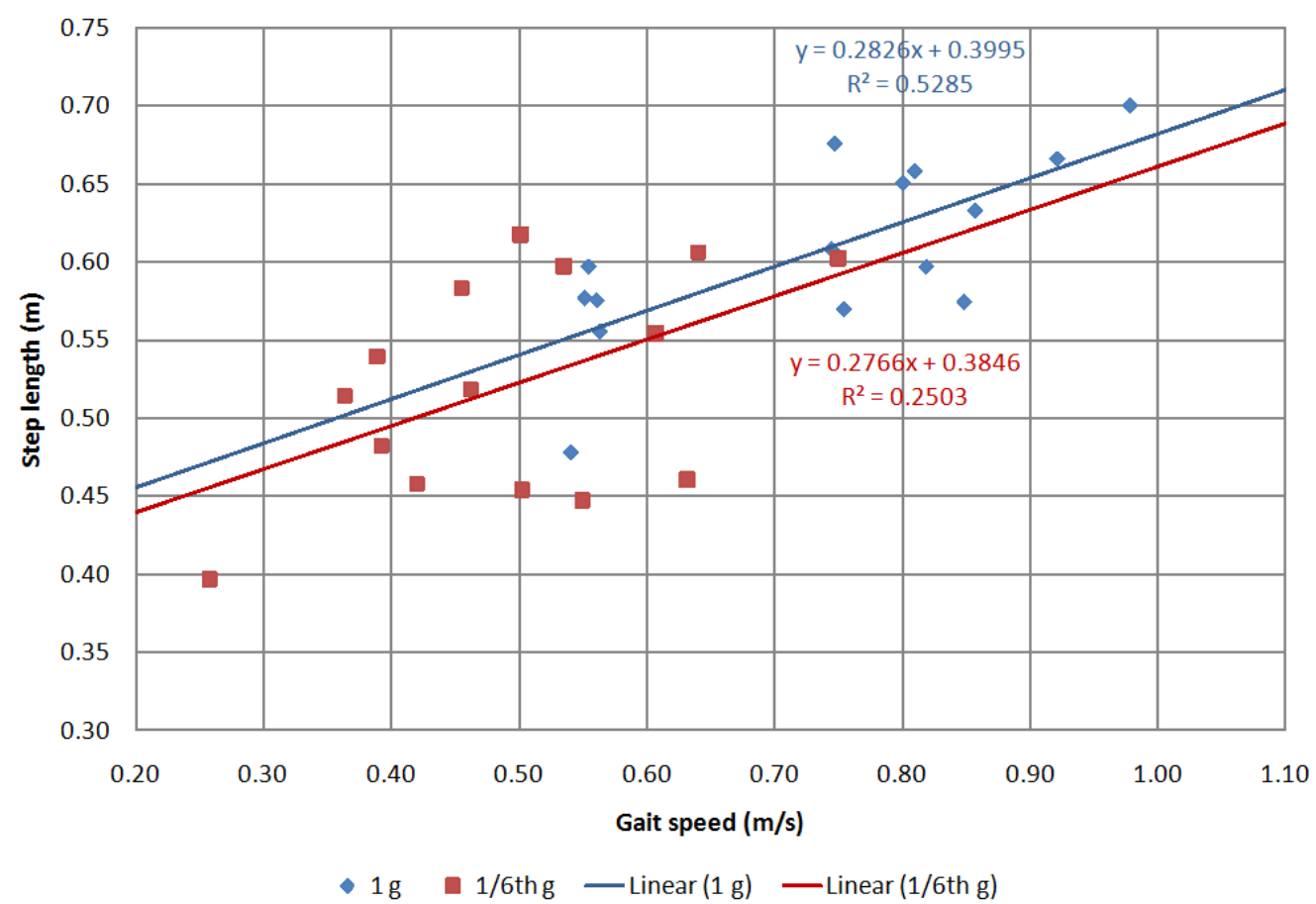


1 g physical, underwater vs. drag-free

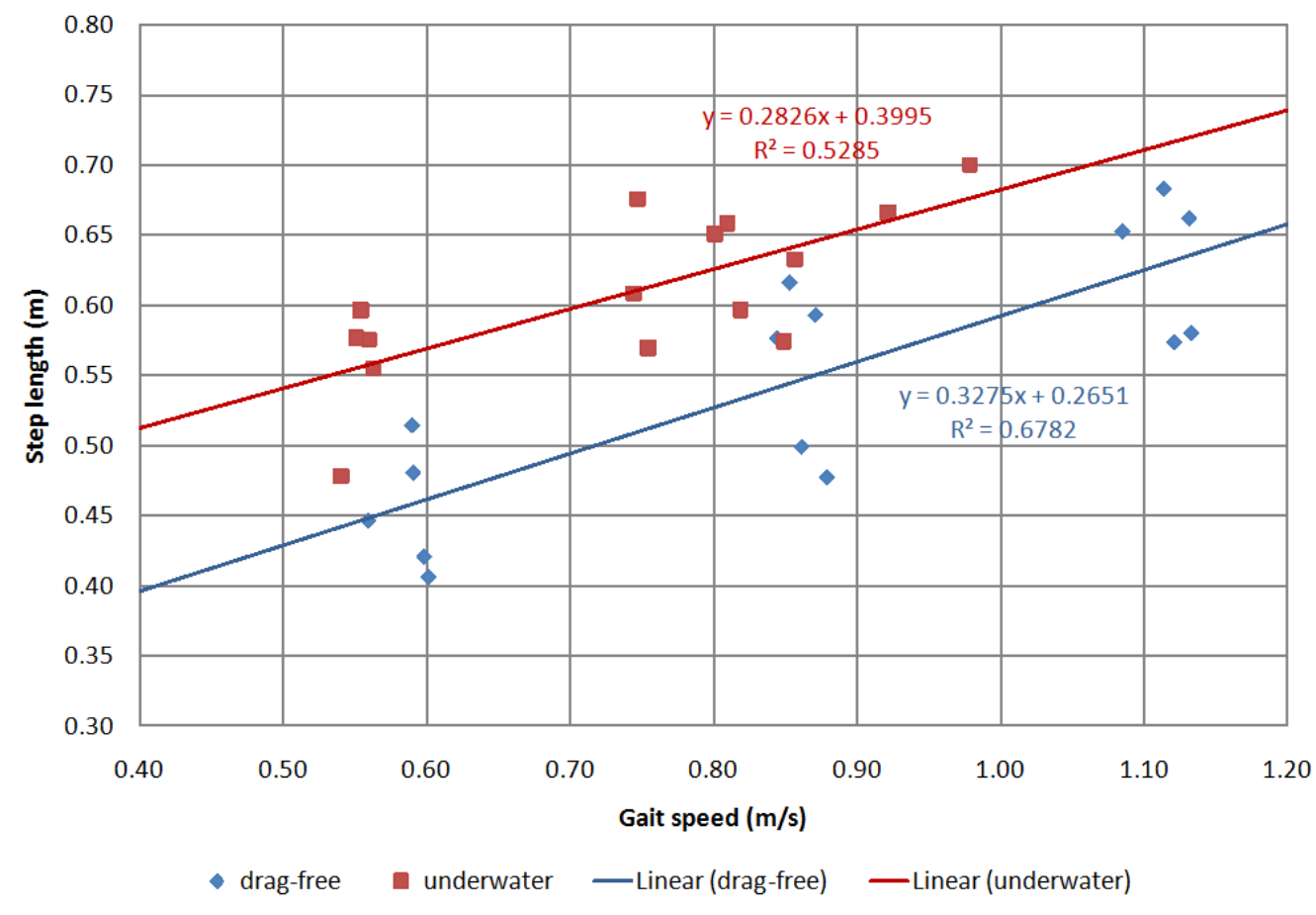


Vertical displacement of the torso

1/6th g model, underwater vs. drag-free

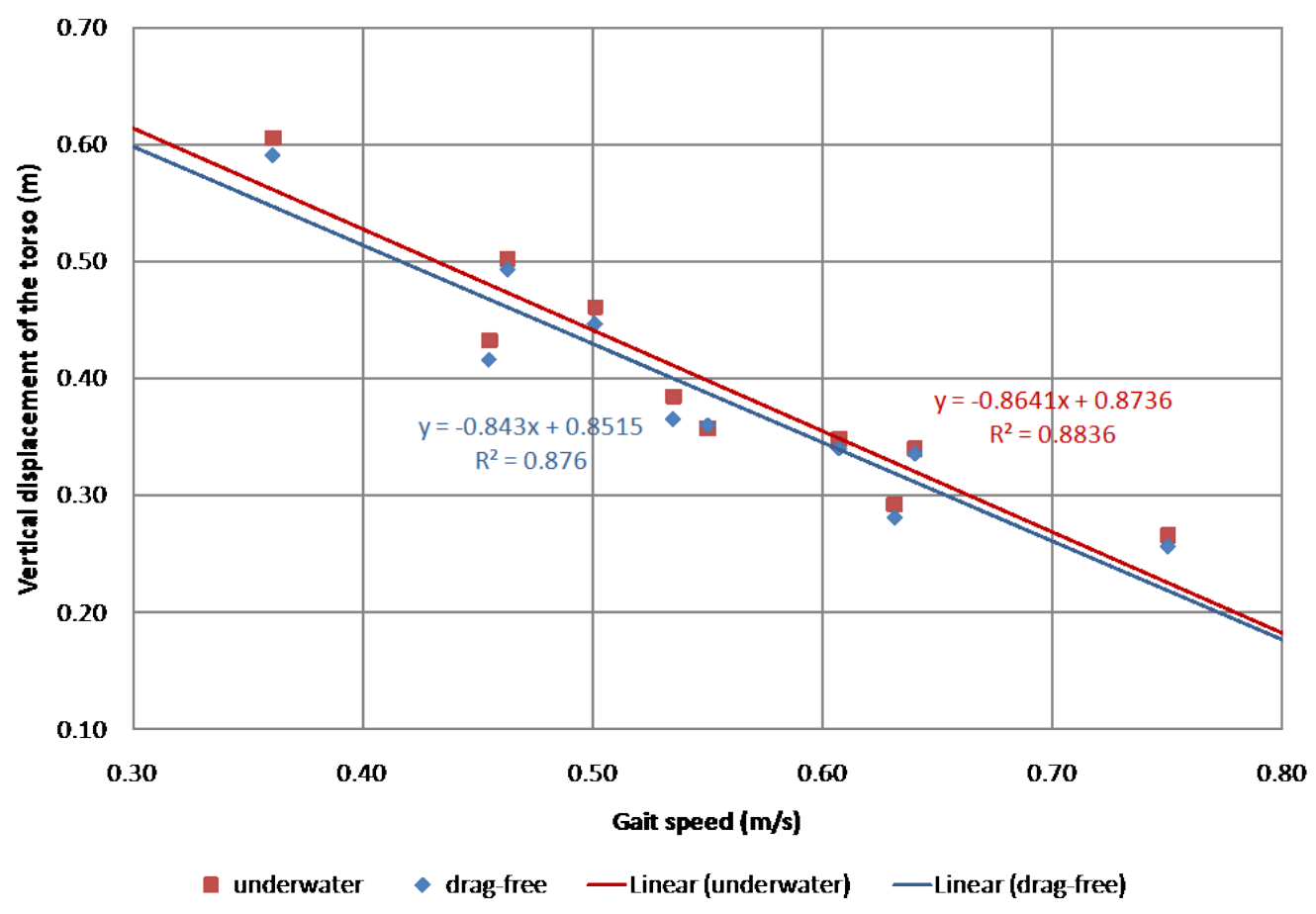

1/6th g underwater, model vs. physical

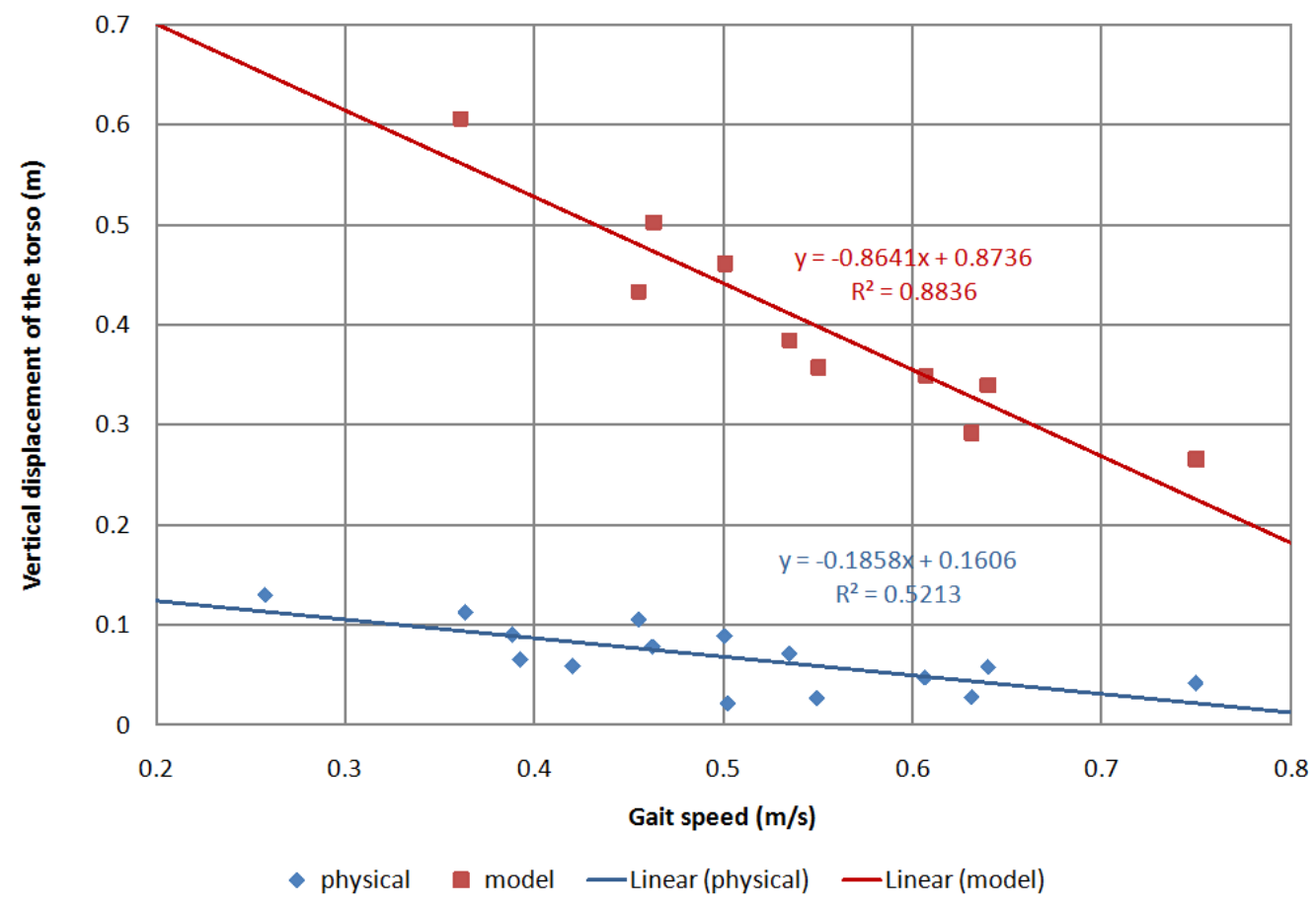


Drag-free model, 1 g vs. 1/6th g

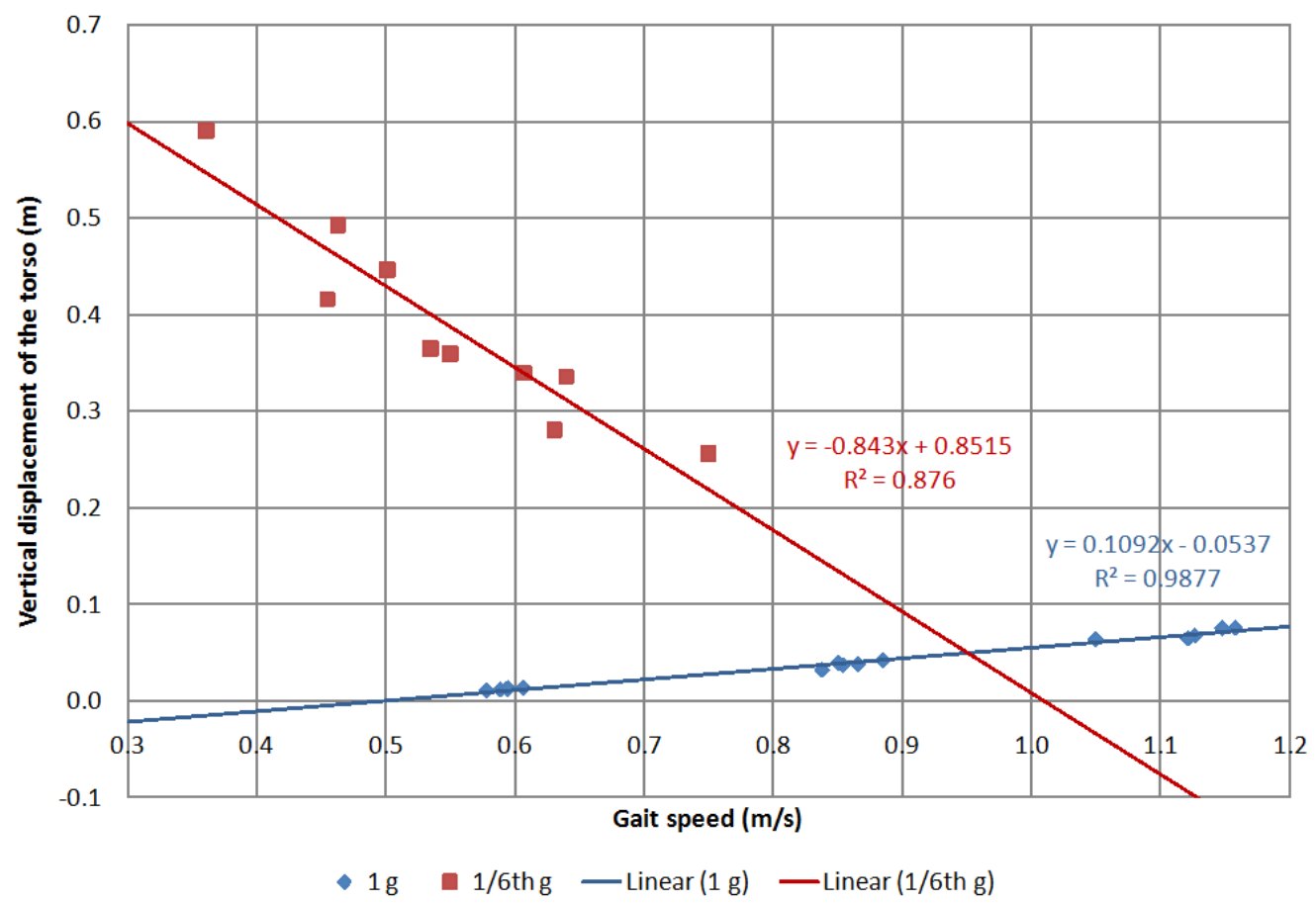

Drag-free 1 g, model vs. physical

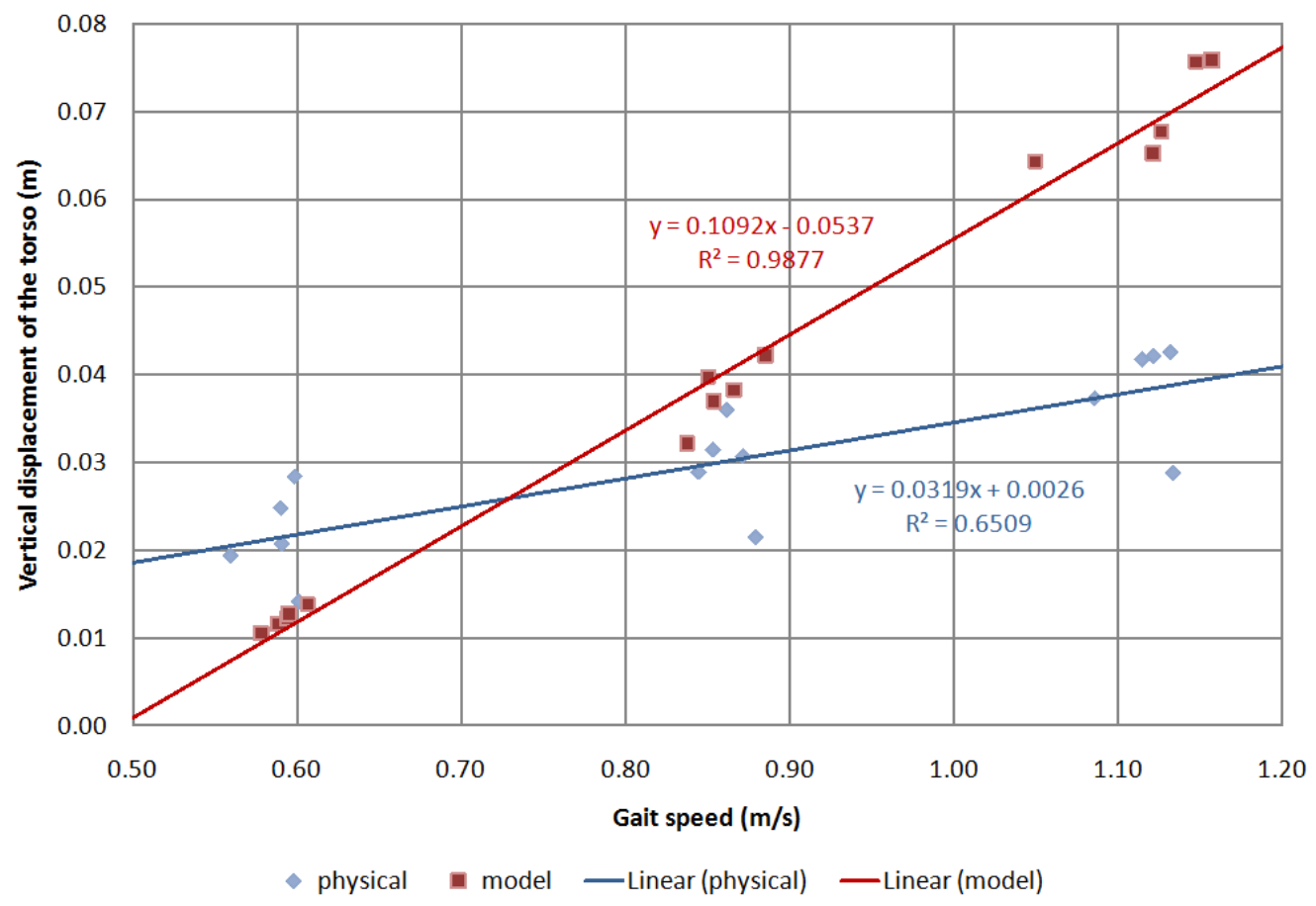


Underwater physical, 1 g vs. 1/6th g

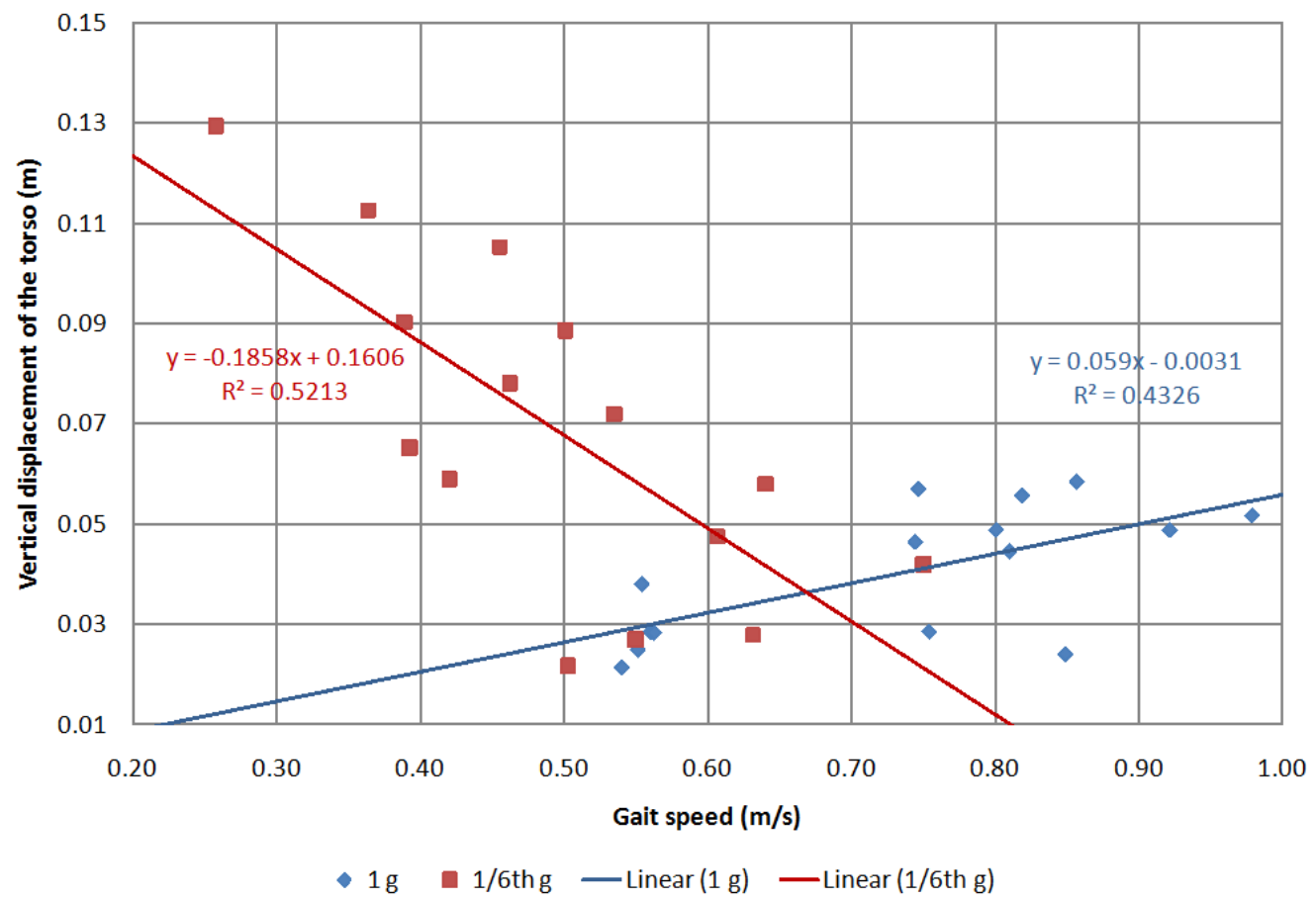

1 g physical, underwater vs. drag-free

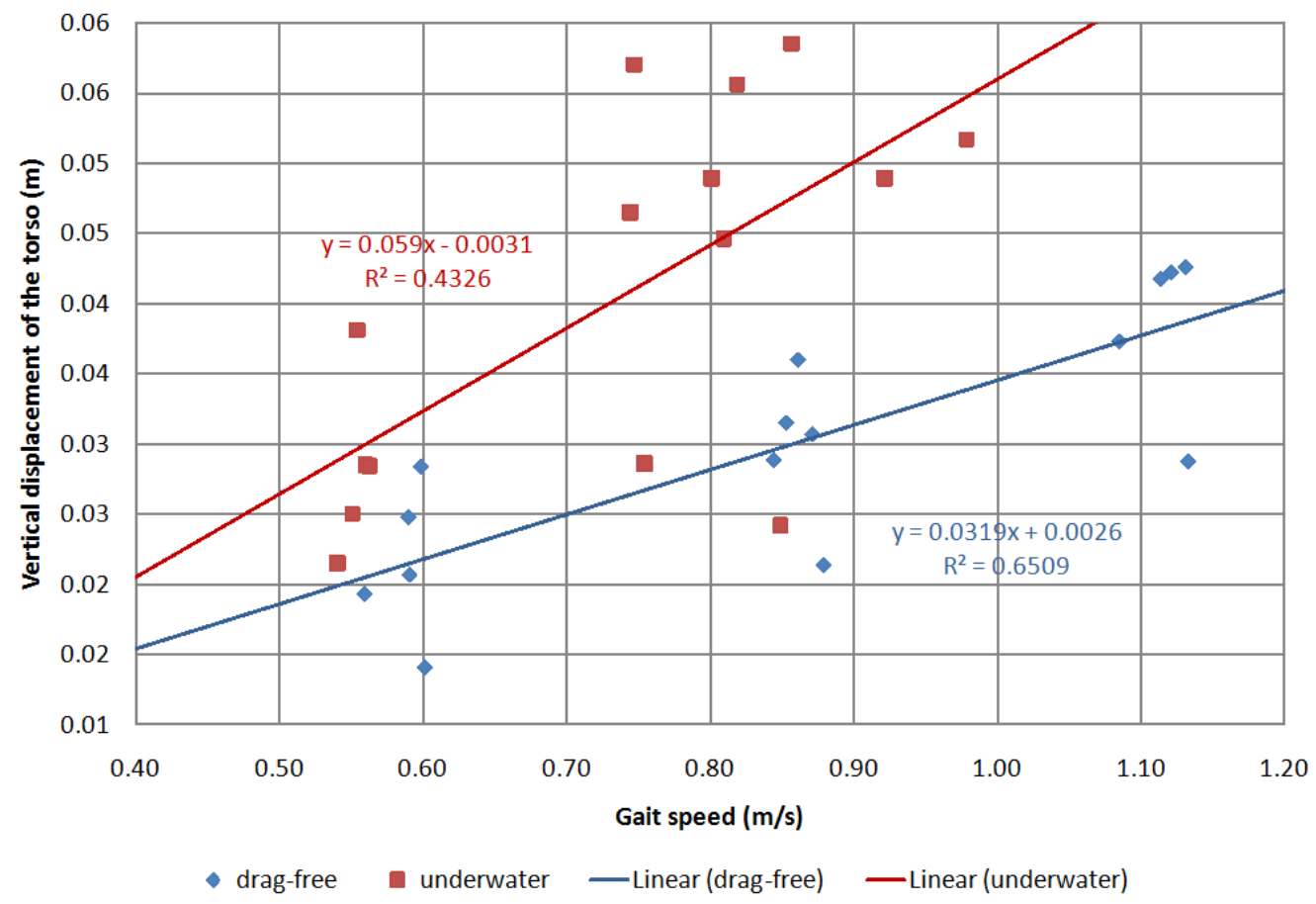


Maximum hip angle

1/6th g model, underwater vs. drag-free

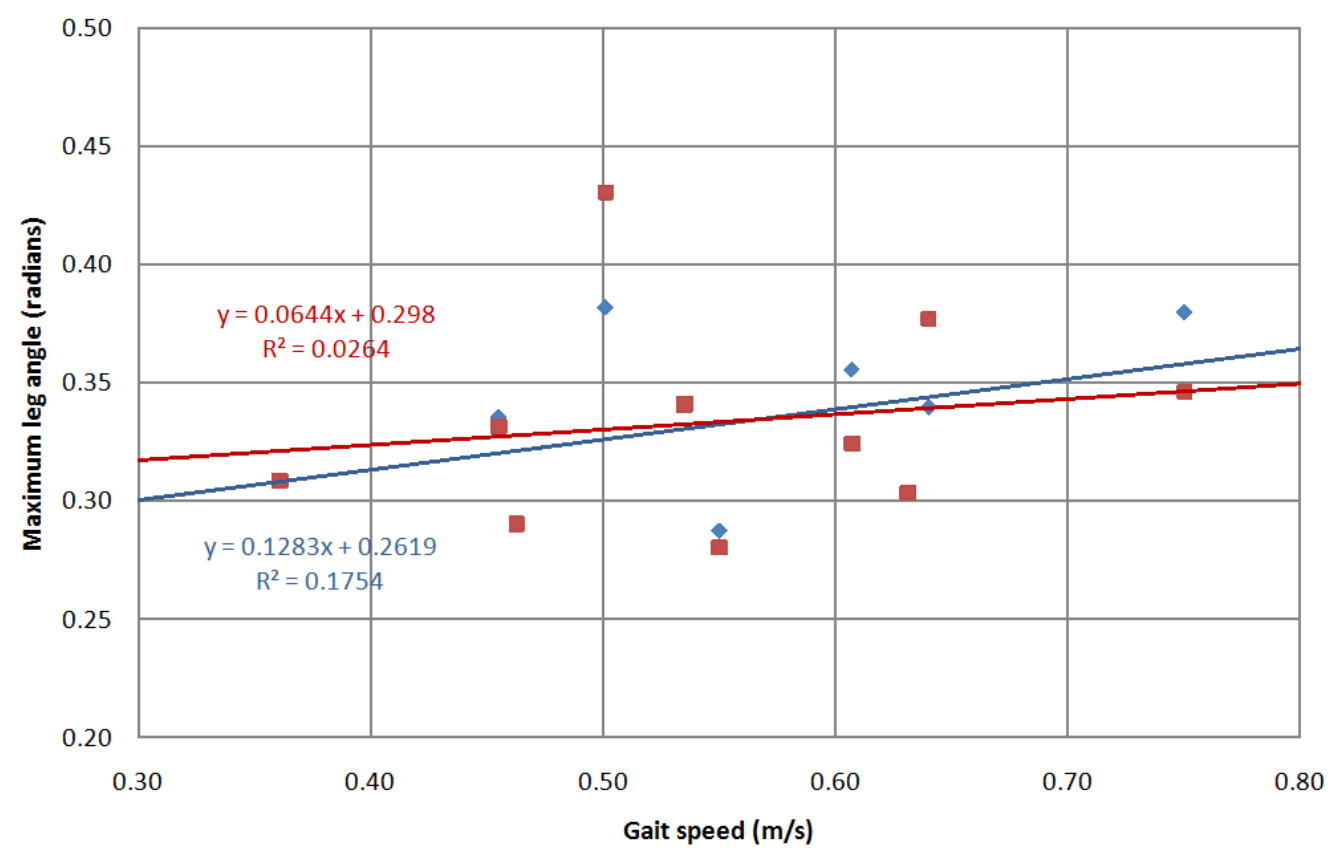

- drag-free underwater — Linear (drag-free) — Linear (underwater)

1/6th g underwater, model vs. physical

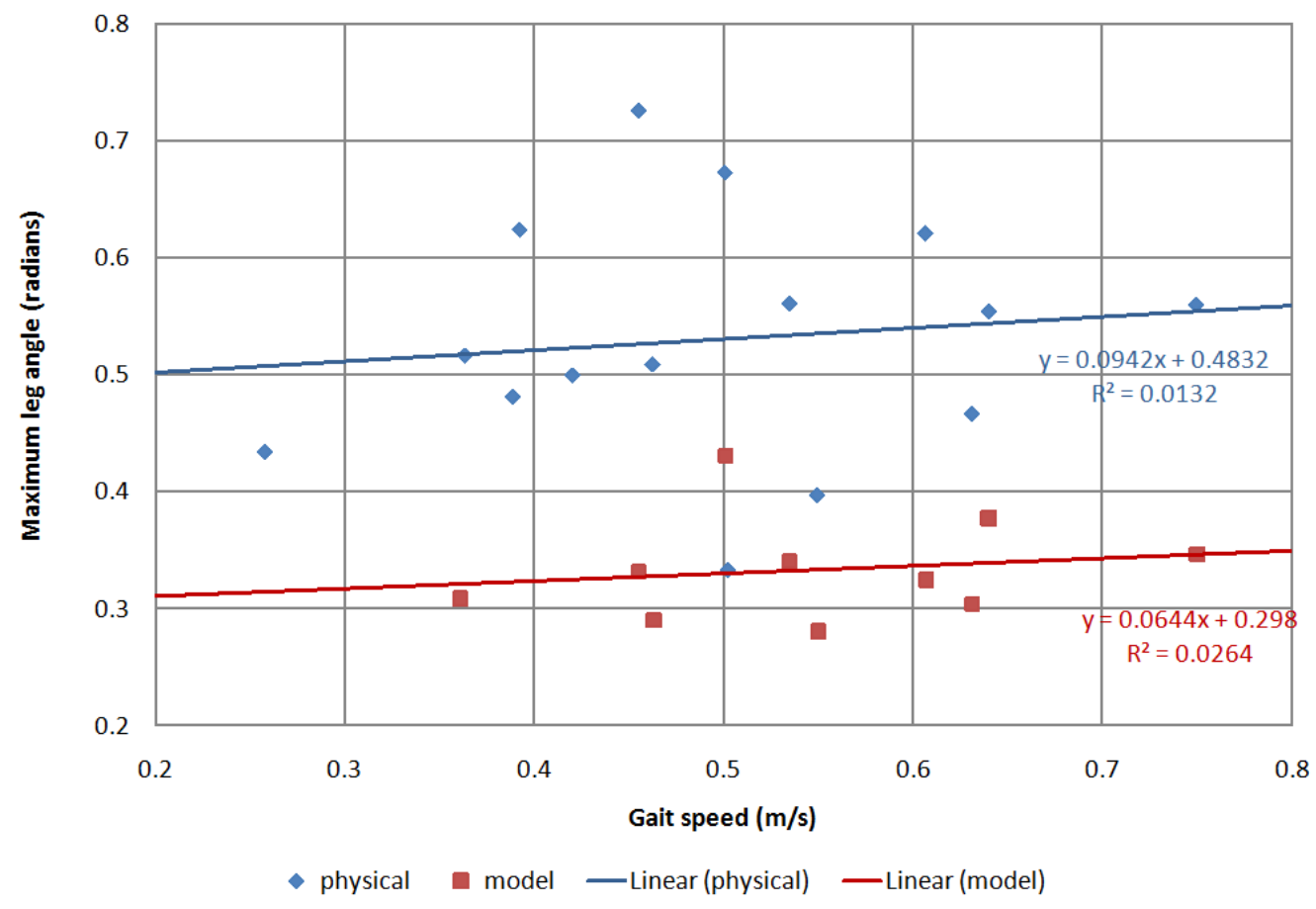


Drag-free model, $1 \mathrm{~g}$ vs. $1 / 6$ th g

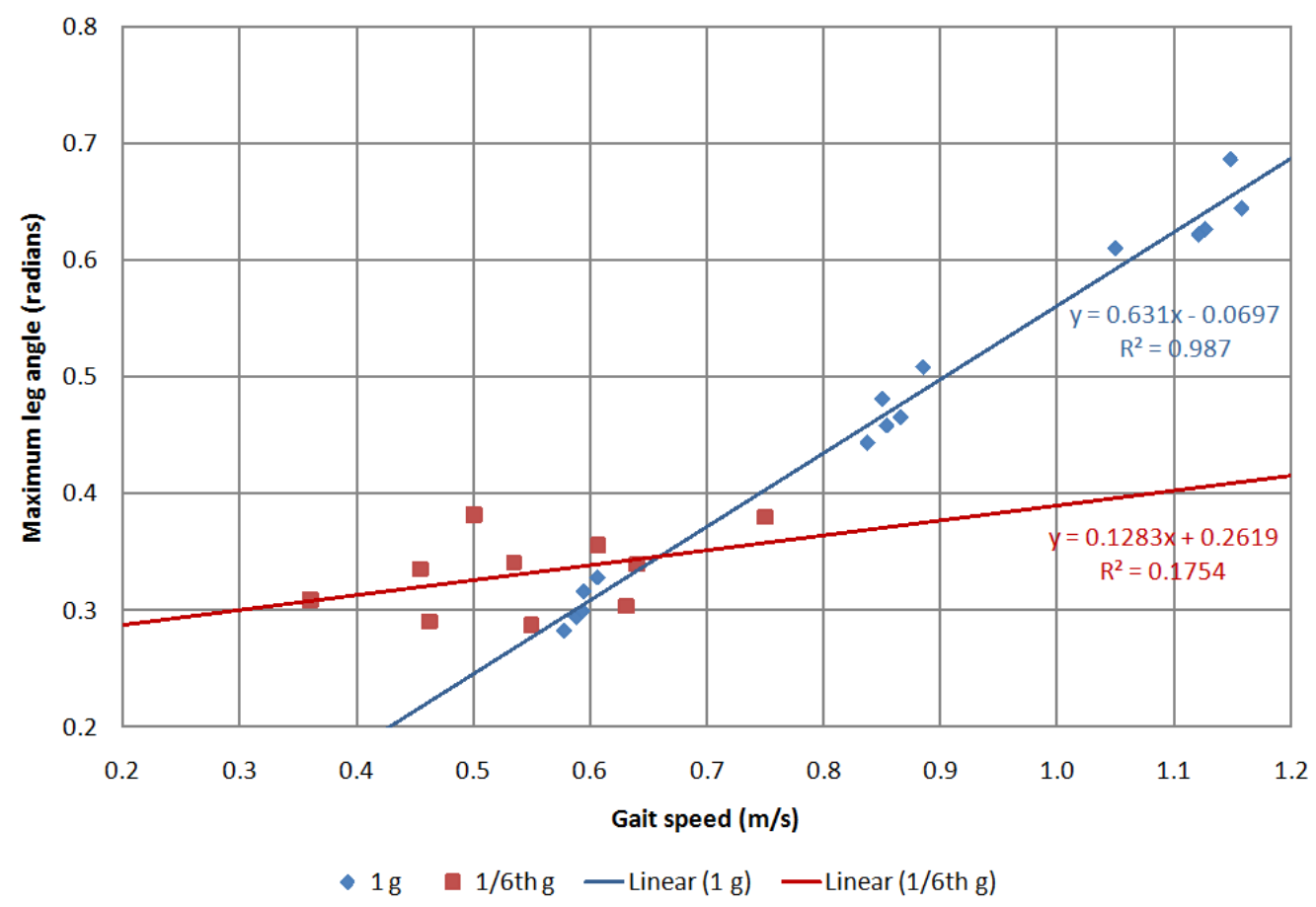

Drag-free 1 g, model vs. physical

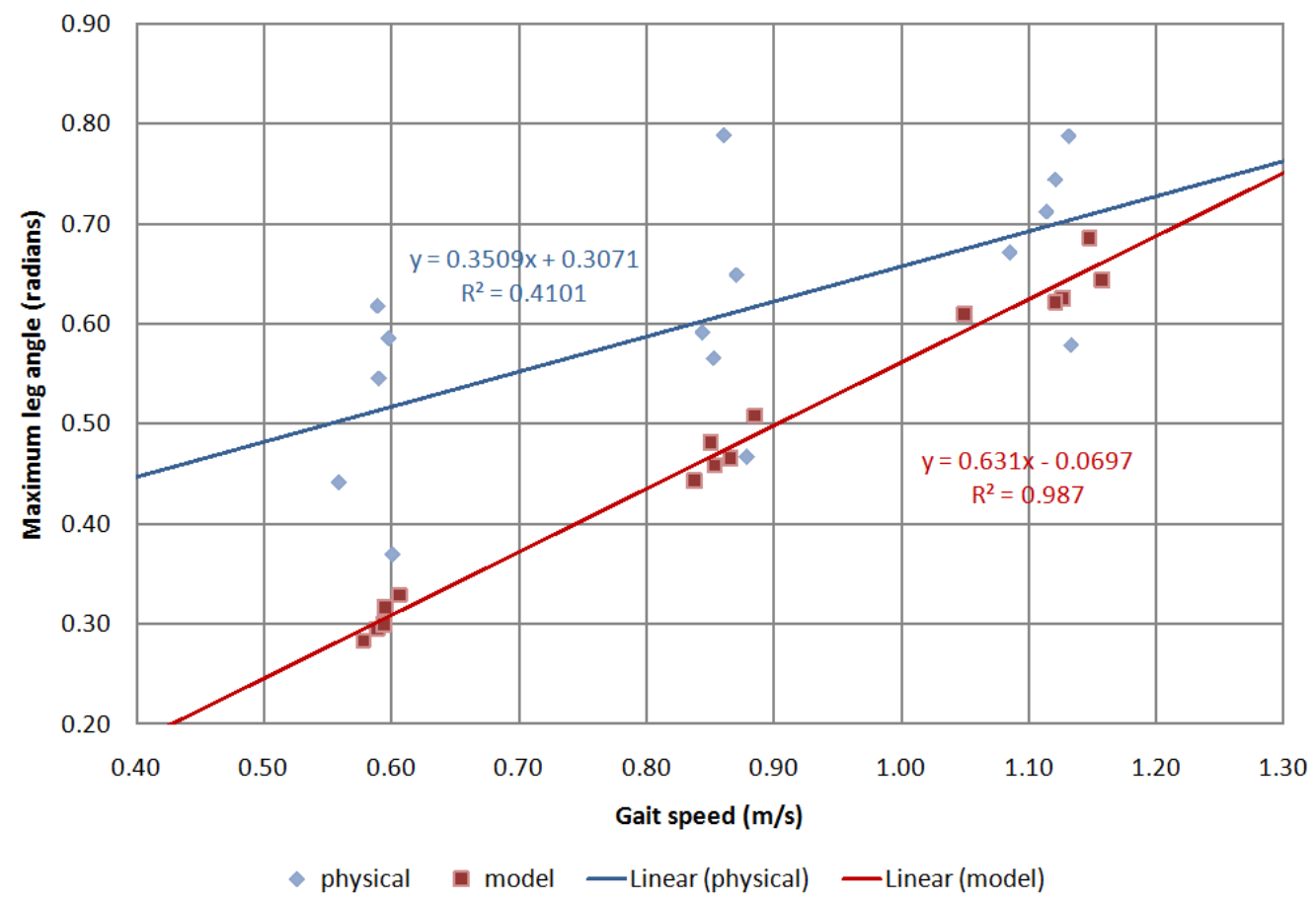


Underwater physical, 1 g vs. 1/6th g

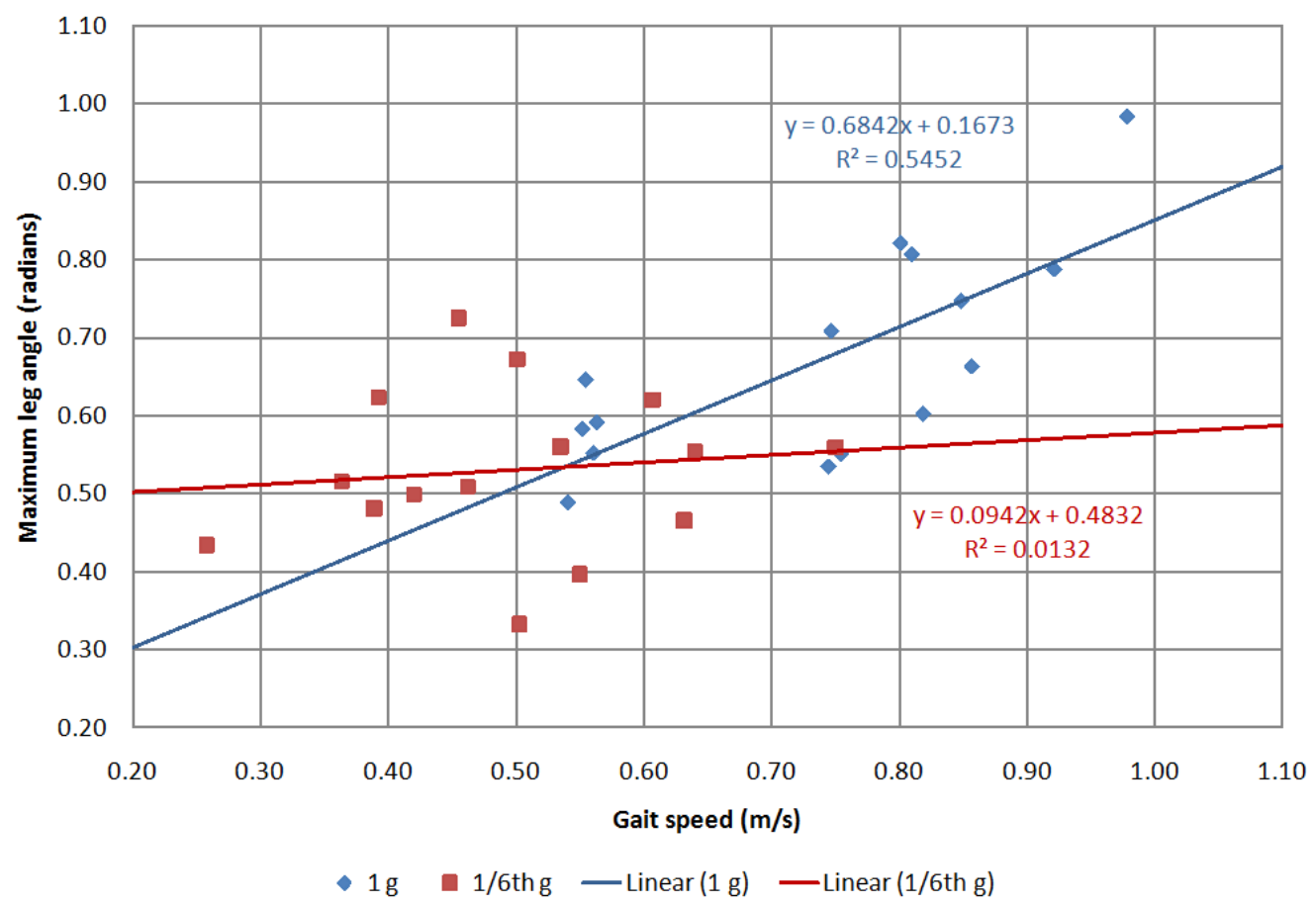

1 g physical, underwater vs. drag-free

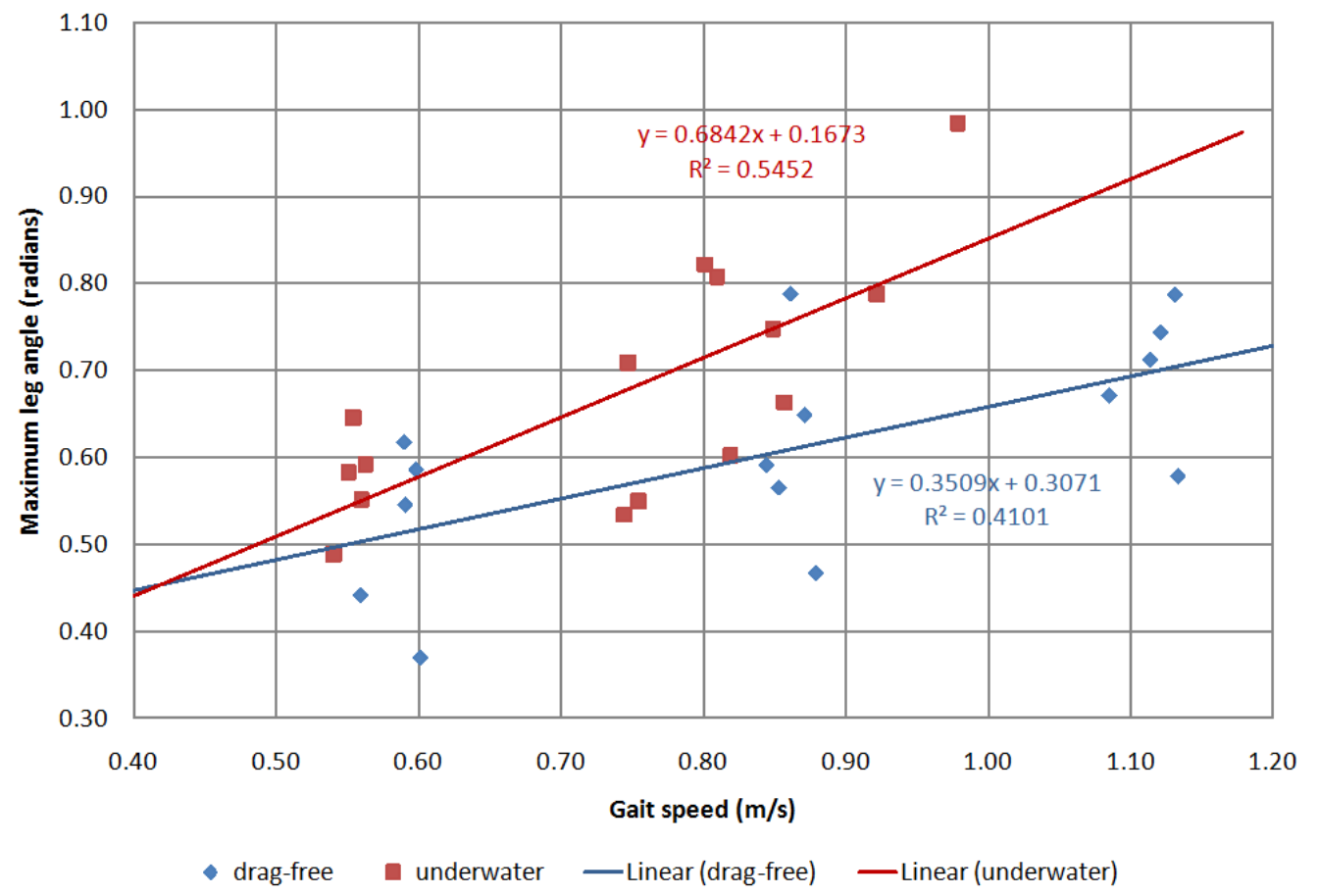


Leg swing frequency

1/6th g model, underwater vs. drag-free

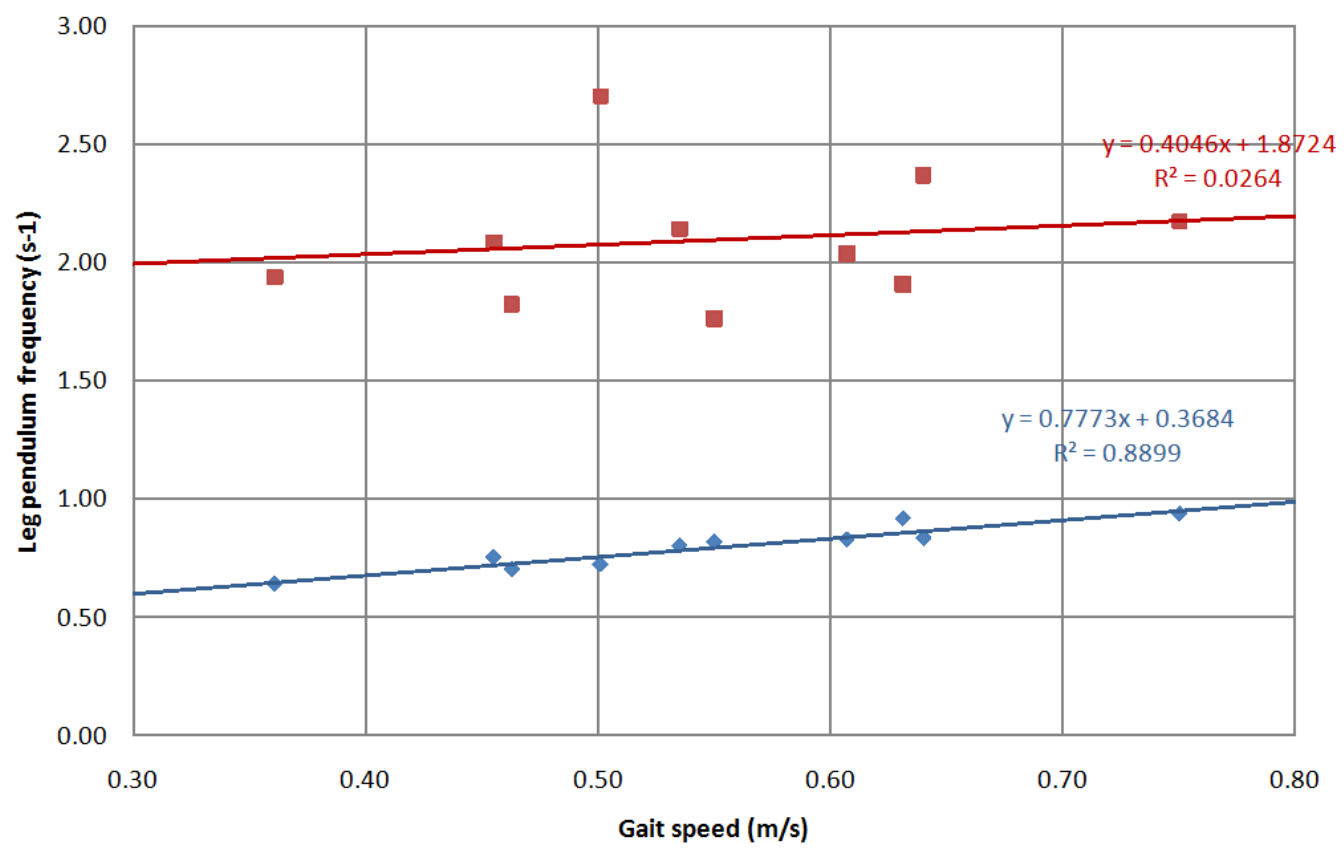

- drag-free $\quad$ underwater — Linear (drag-free) — Linear (underwater)

$1 / 6$ th g underwater, model vs. physical

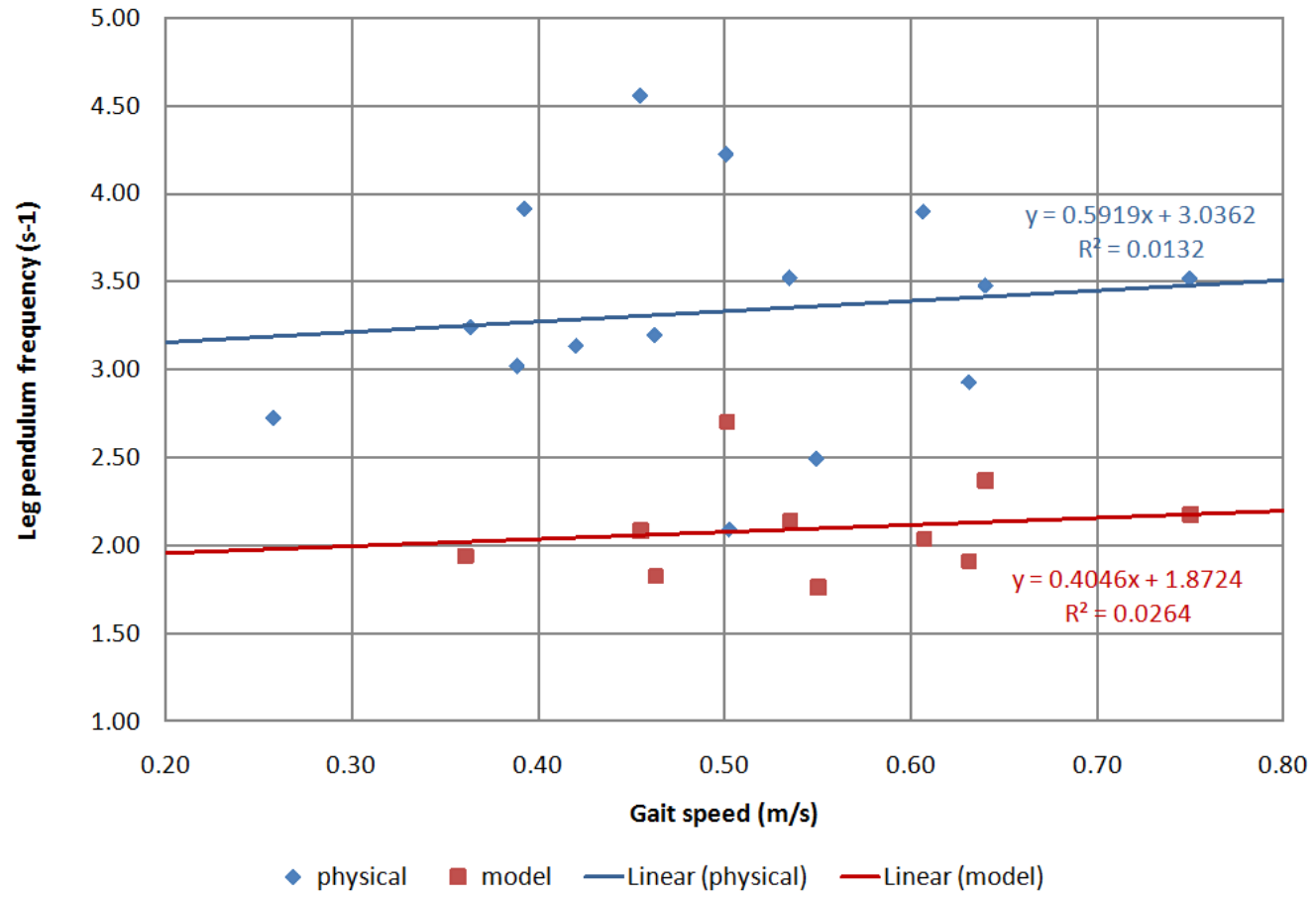


Drag-free model, 1 g vs. 1/6th g

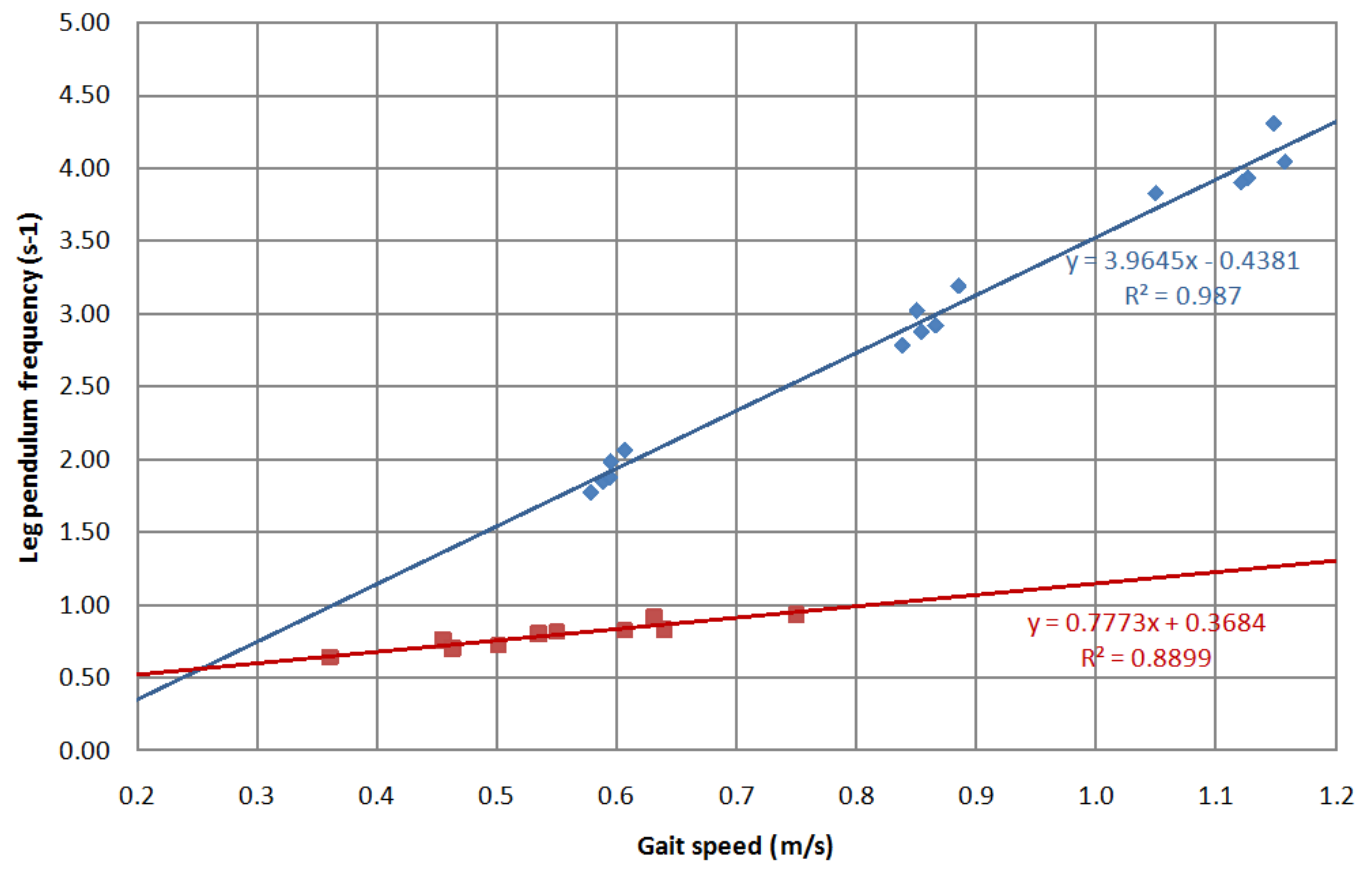

- $1 \mathrm{~g} \square$ 1/6th $\mathrm{g}$ - Linear $(1 \mathrm{~g})$ —Linear (1/6th g)

Drag-free $1 \mathrm{~g}$, model vs. physical

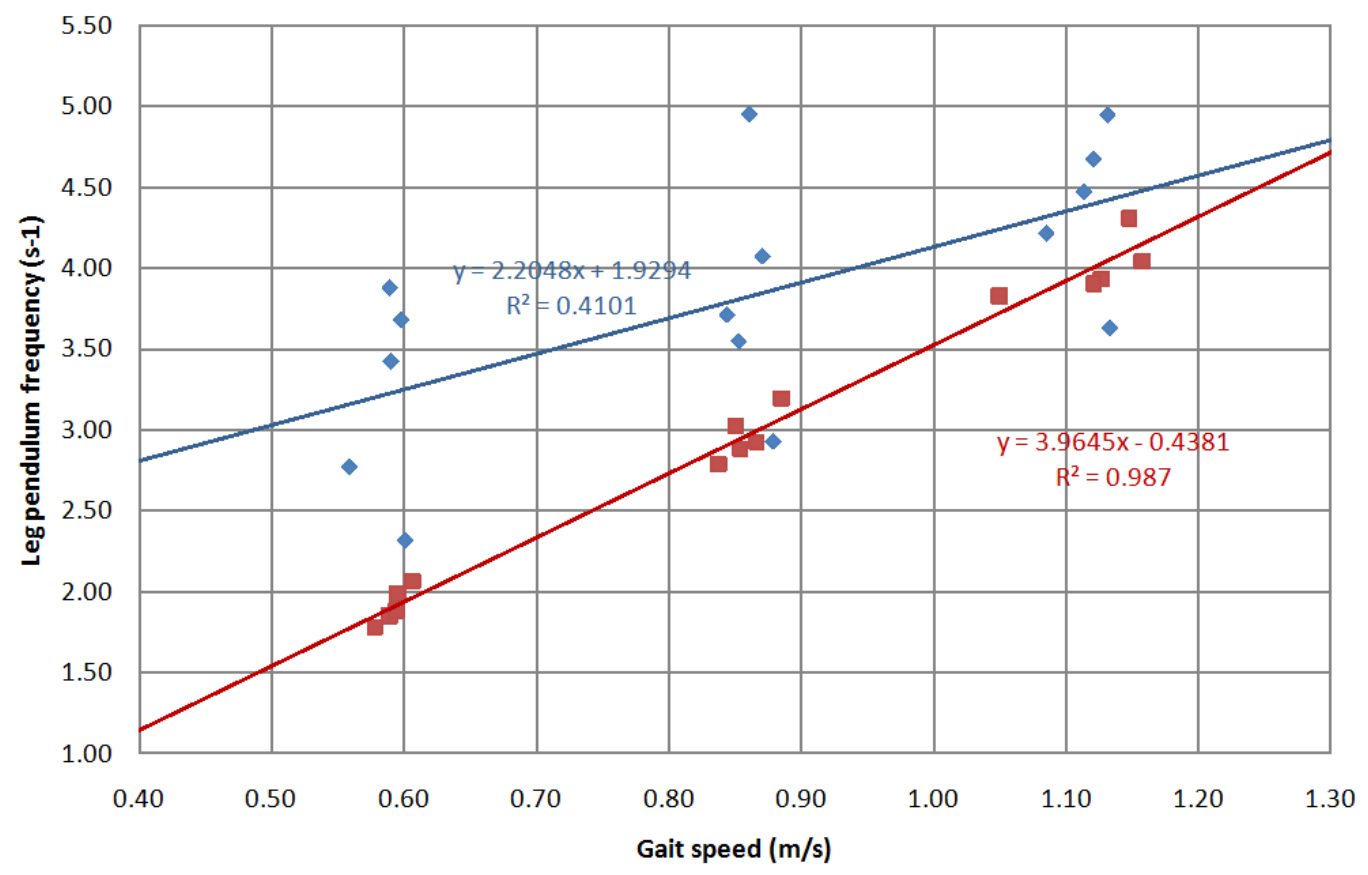

- physical model —Linear (physical) —Linear (model) 
Underwater physical, 1 g vs. 1/6th g

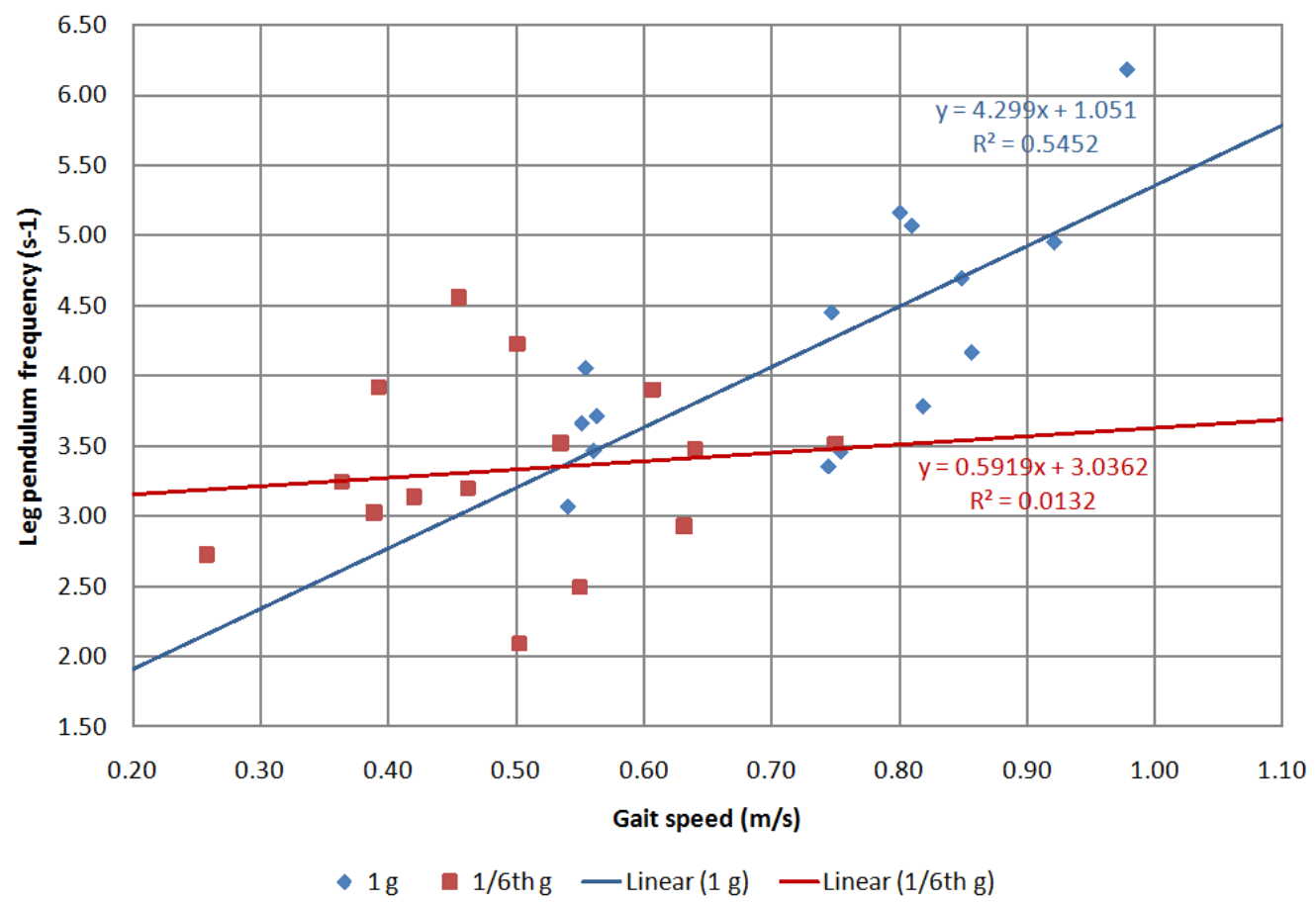

1 g physical, underwater vs. drag-free

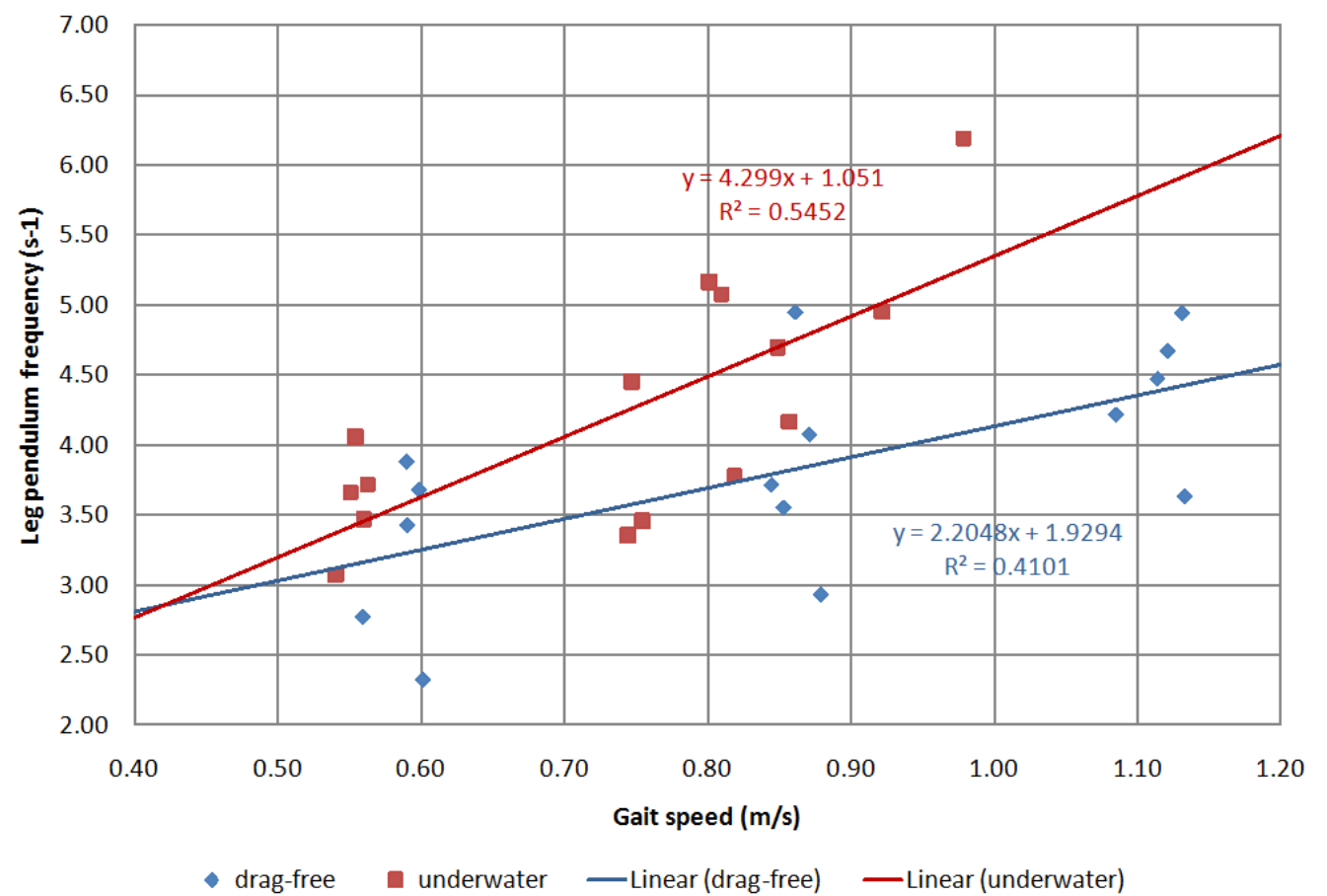


Appendix 3: Extended test matrix-difference plots

The following plots estimate the impact of each environmental factor (gravity, water submersion, and modeling limitations) on the gait metric functions. The regression fits identified in Appendix 3 are used. The plots show the weighted and un-weighted averages for each factor and each gait metric. The following shorthand is used in the legend of each plot to identify the two estimates:

$$
\begin{aligned}
& \mathrm{s}=\text { step length } \\
& \mathrm{t}=\text { vertical displacement of torso } \\
& \mathrm{p}=\text { maximum angle between legs } \\
& \mathrm{o}=\text { leg pendulum frequency }
\end{aligned}
$$

In thi manner, the label "sEG", for example, represents the normalized difference in step length between node $E$ (underwater physical test data in $1 / 6^{\text {th }} \mathrm{g}$ ) and node $\mathrm{G}$ (underwater physical test data in $1 \mathrm{~g}$ ) - i.e., sE minus sG. 


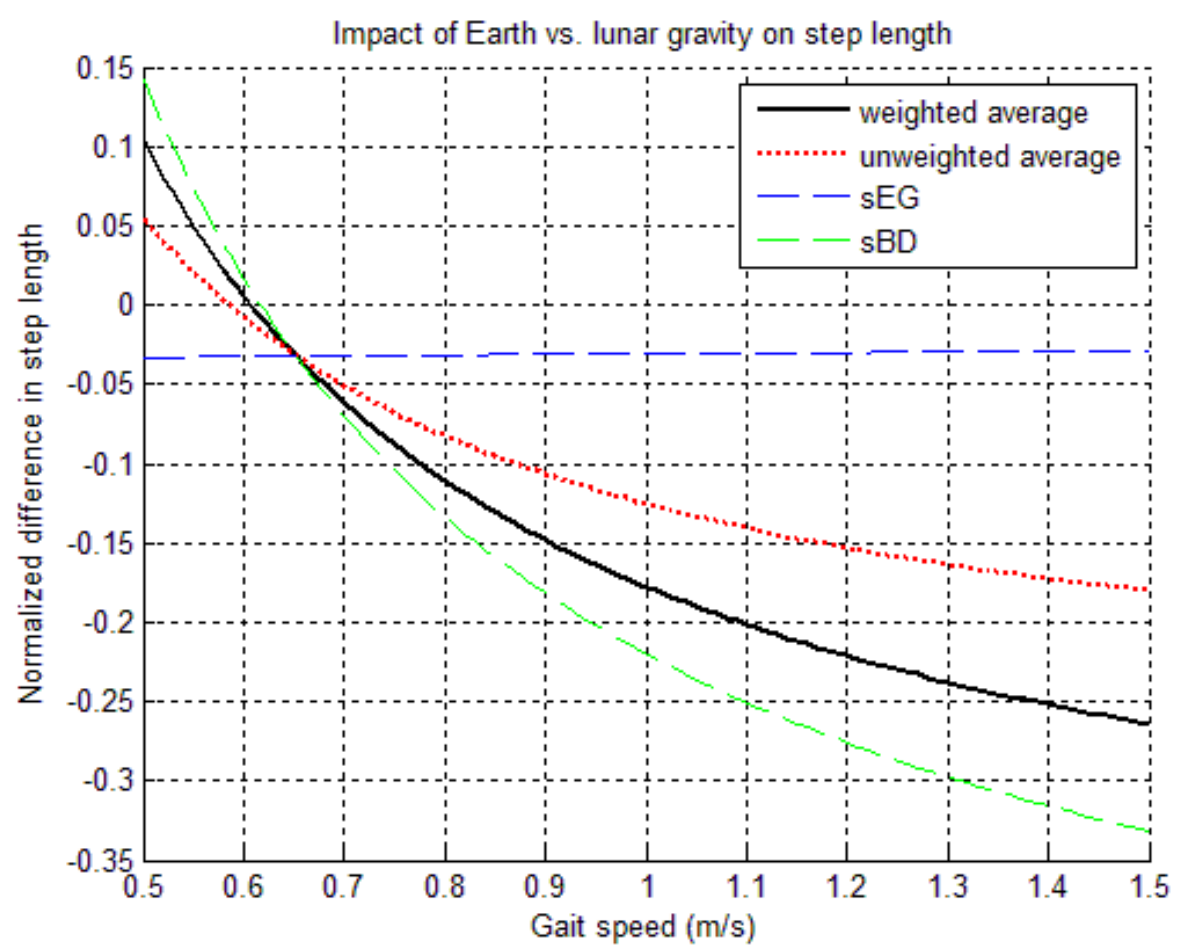

Figure 34: gravity effect on step length (extended test matrix)

In this first plot, note that for a gait speed of approximately $0.6 \mathrm{~m} / \mathrm{s}$, the impact of gravity level on step length is approximately zero. For slower speeds, step length is greater in lunar gravity, and for faster speeds, step length is greater in Earth gravity. Below speeds of $1.4 \mathrm{~m} / \mathrm{s}$, the difference in step length between the two gravity levels is less than about $25 \%$. 


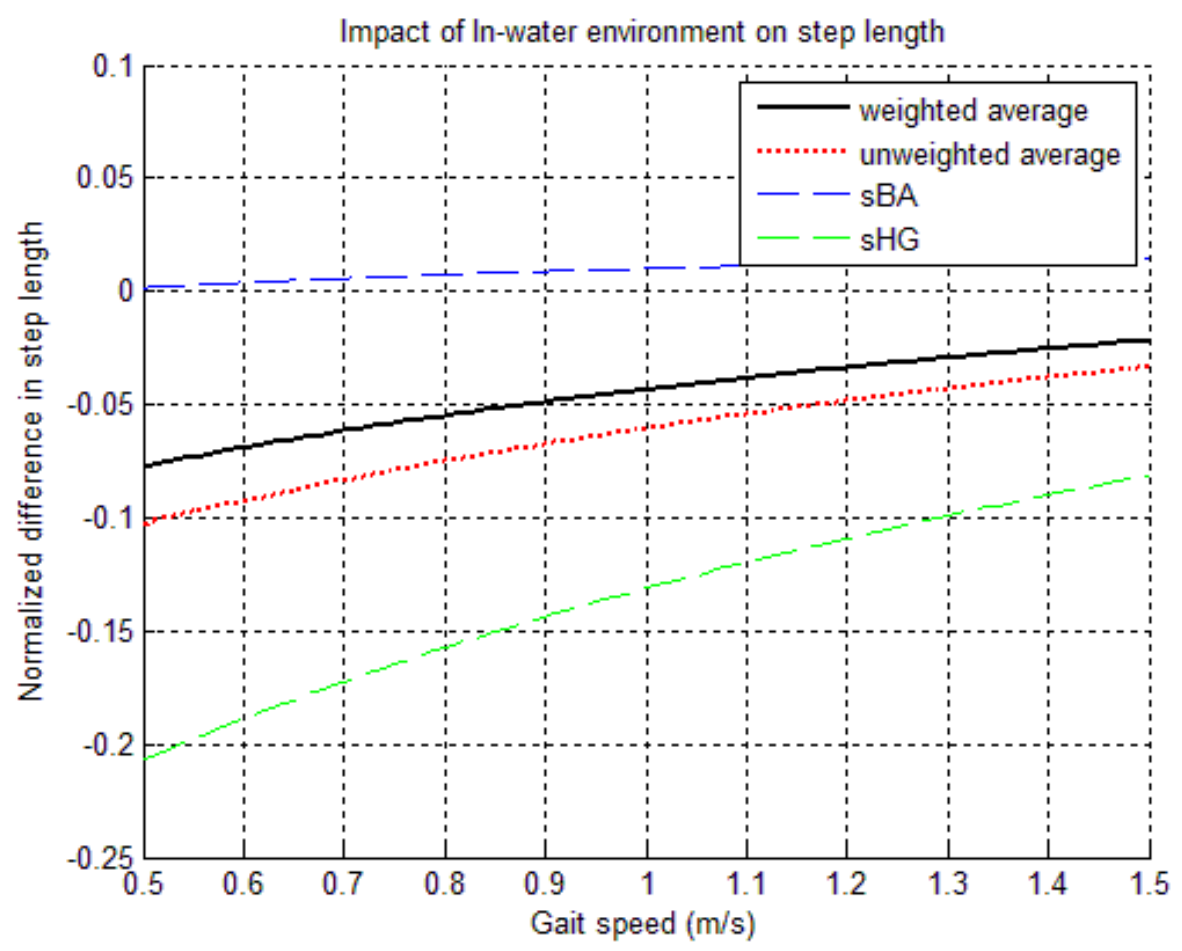

Figure 35: In-water effect on step length (extended test matrix)

The weighted average in this plot indicates that step length is greater in underwater environments than in drag-free environments, with the difference greater at lower speeds. Over the range of speeds considered, the difference is less than about $7 \%$. 


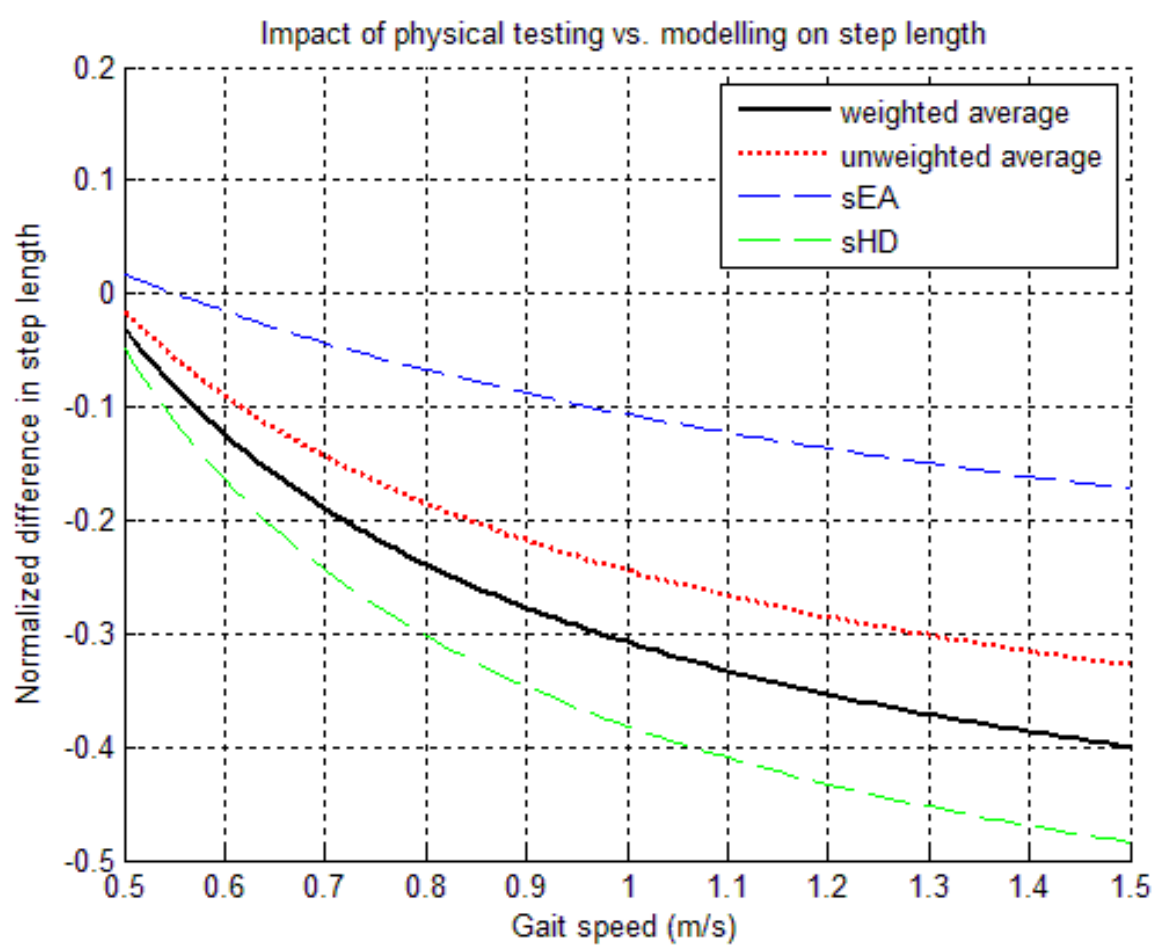

Figure 36: Modelling effect on step length (extended test matrix)

This weighted average indicates that step length is greater in the models than in the physical data, with the difference increasing with speed. At $1.4 \mathrm{~m} / \mathrm{s}$, step length is approximately $38 \%$ greater in the models than in the physical data. 


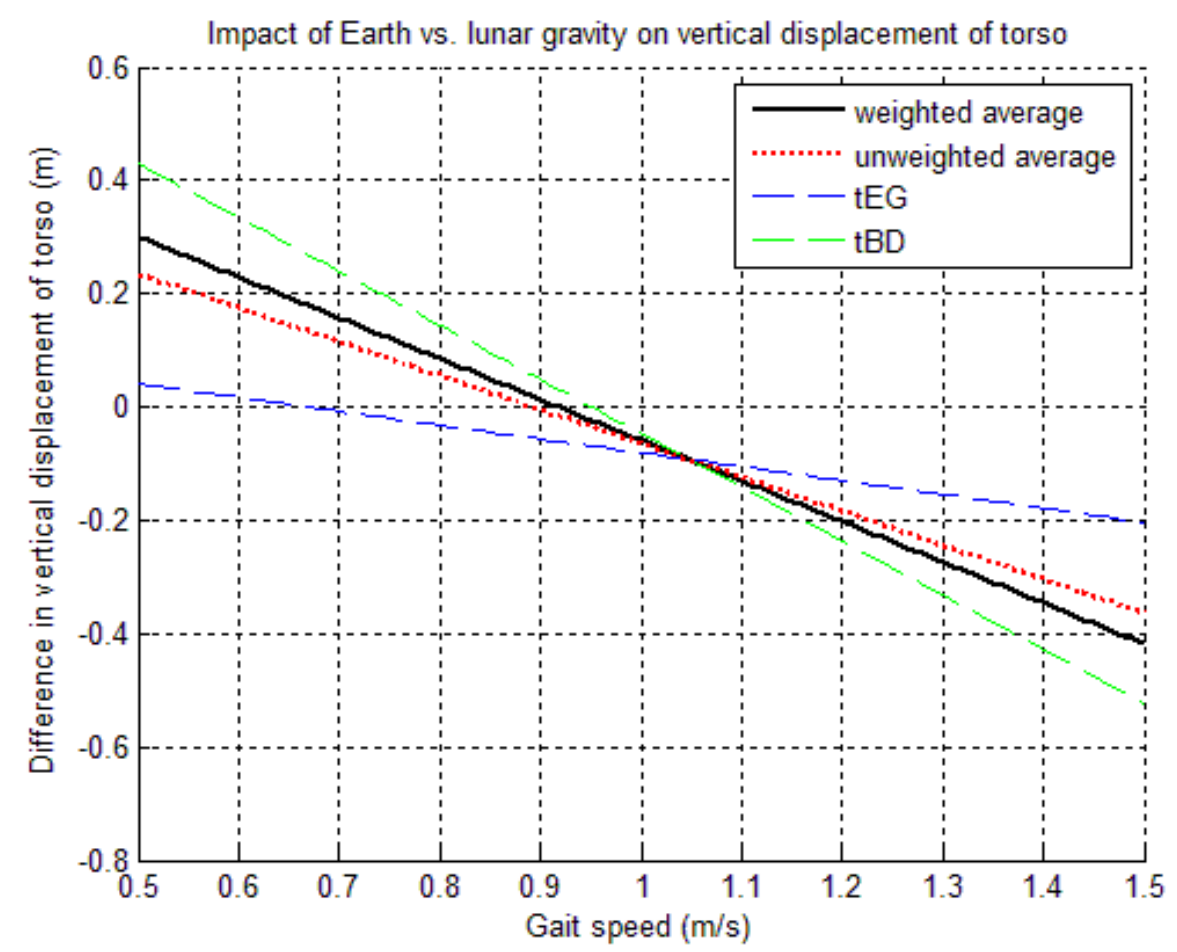

Figure 37: Gravity impact on torso displacement (extended test matrix)

This weighted average indicates that for speeds around $0.9 \mathrm{~m} / \mathrm{s}$, the impact of gravity level on vertical displacement of the torso is approximately zero. For slower speeds, greater displacement is seen in lunar gravity; at faster speeds, greater displacement is seen in Earth gravity. 


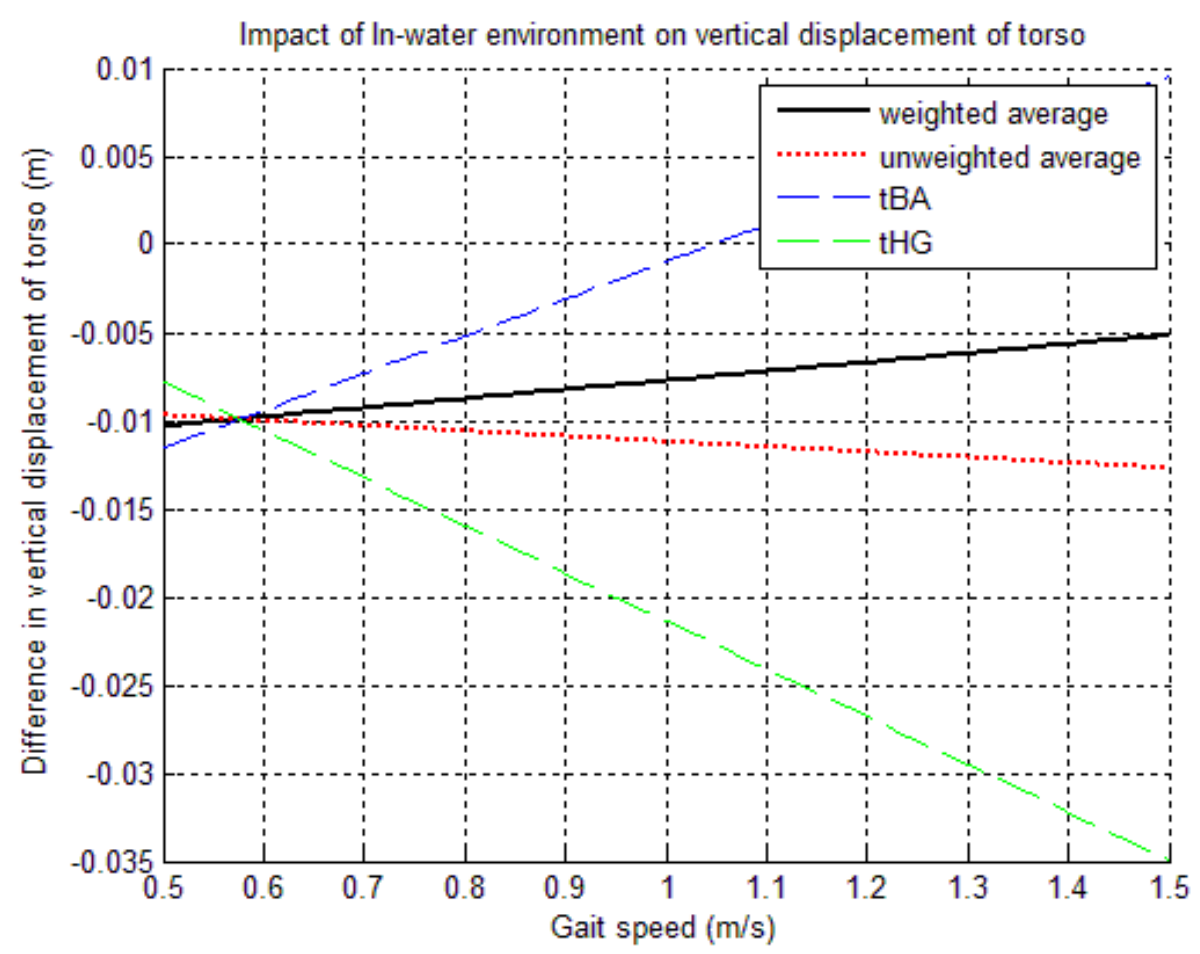

Figure 38: In-water affect on torso displacement (extended test matrix)

This plot indicates that the impact of the in-water environment on vertical displacement of the torso is negligible. Note that the lunar gravity models indicate that faster speeds correspond to a greater displacement in a drag-free environment relative to an underwater environment, while the physical data in $1 \mathrm{~g}$ suggest the opposite, with faster speeds indicating a greater displacement in underwater environments relative to drag-free environments. 


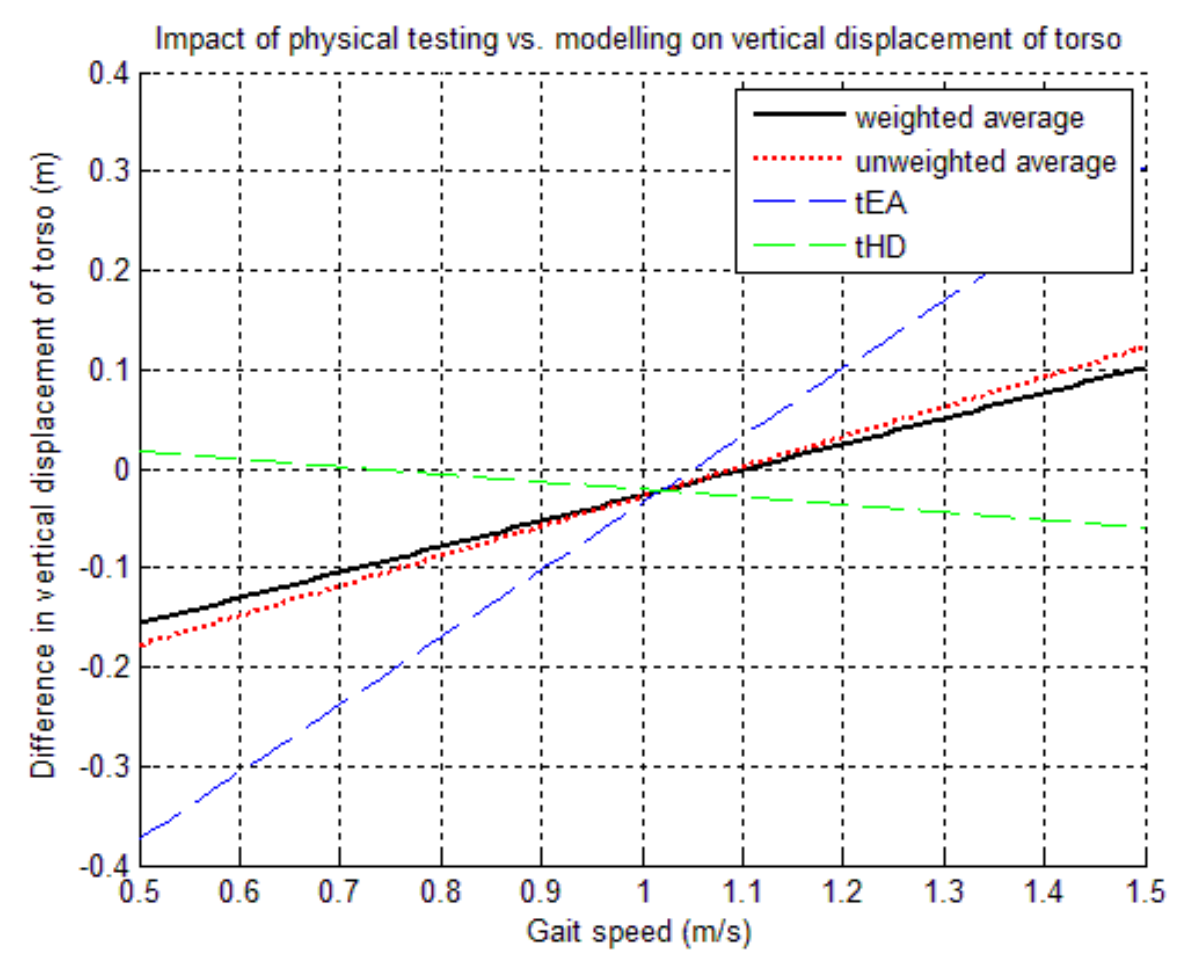

Figure 39: Modelling impact on torso displacement (extended test matrix)

This weighted average indicates that for gait speeds around $1.1 \mathrm{~m} / \mathrm{s}$, the physical data and virtual model data produce similar estimates for vertical displacement of the torso. For slower speeds, the models predict a greater displacement than is shown in the physical data, and at higher speeds, the models predict a smaller displacement. 


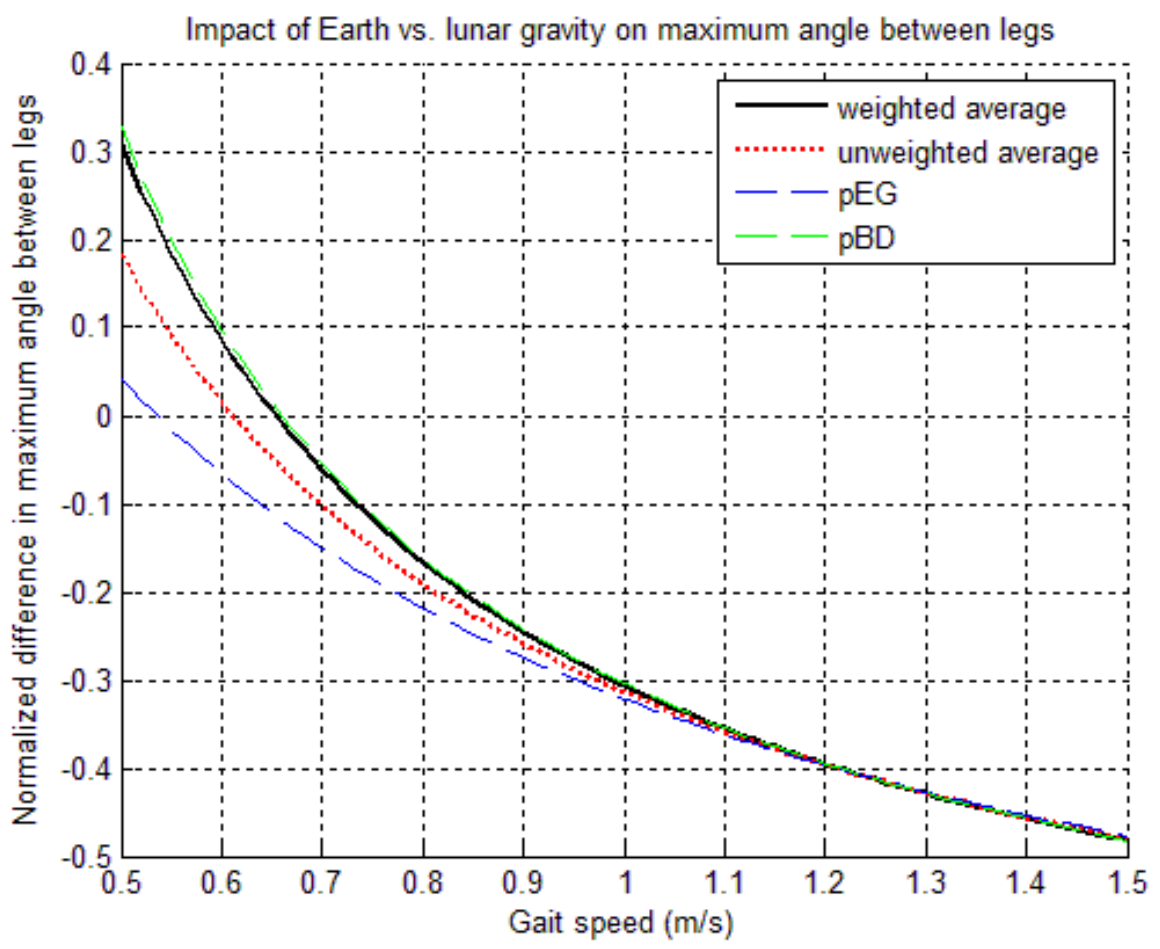

Figure 40: gravity impact on hip angle (extended test matrix)

This weighted average indicates that for gait speeds around $0.65 \mathrm{~m} / \mathrm{s}$, the maximum angle between the legs during each stride is not affected by gravity level. For slower speeds, larger leg angles are expected in lunar gravity; at faster speeds, larger leg angles are expected in Earth gravity. 


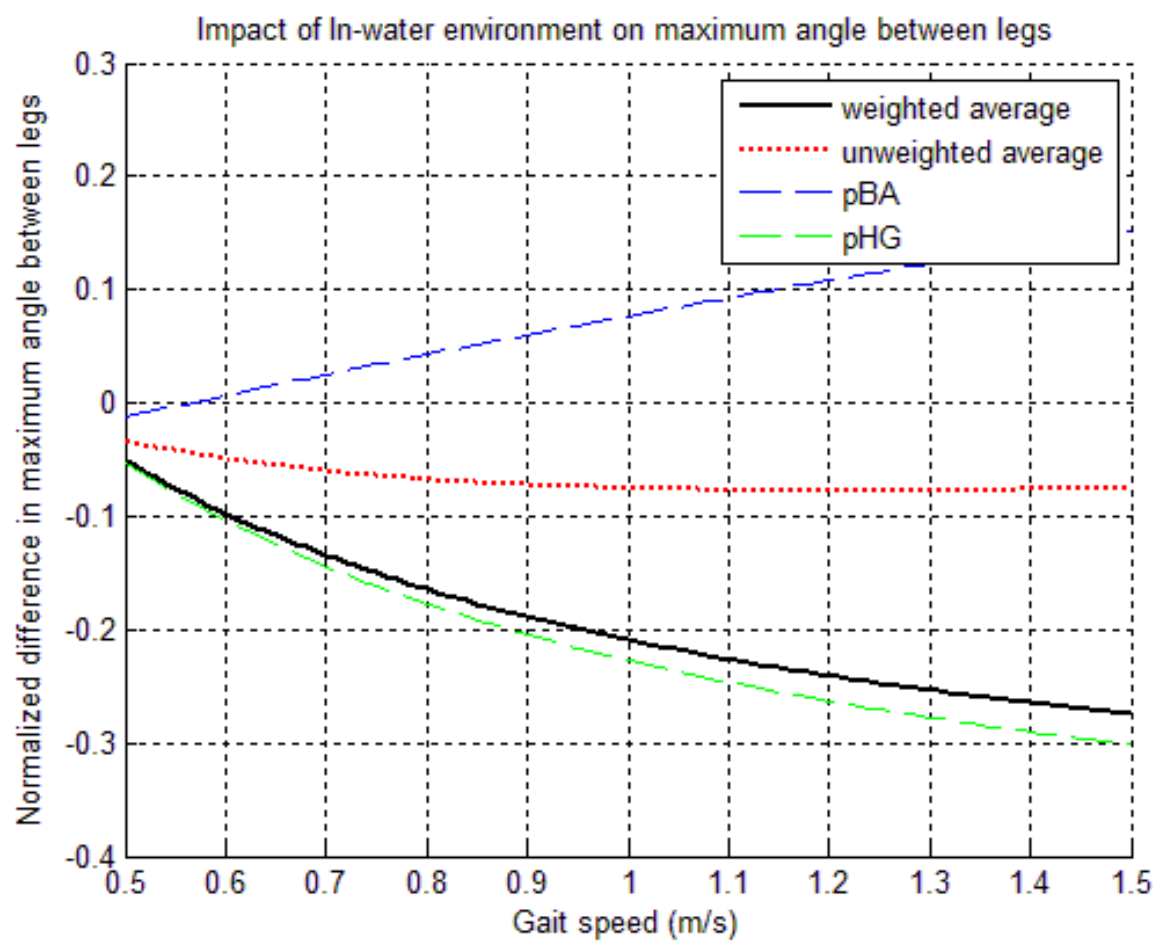

Figure 41: In-water impact on hip angle (extended test matrix)

This weighted average indicates that over the considered range of gait speeds, larger leg angles are seen in underwater environments than in drag-free environments, with this difference increasing with speed. 


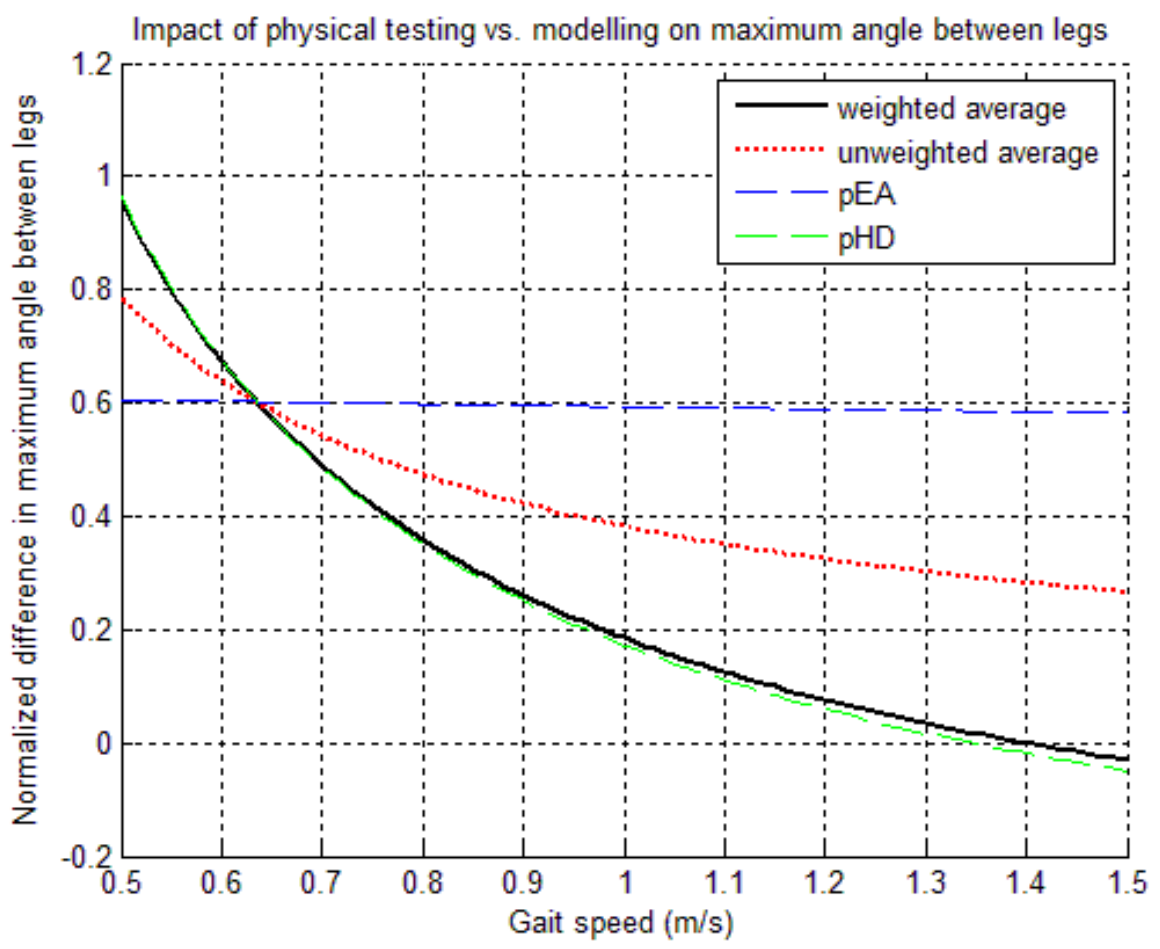

Figure 42: Modelling impact on hip angle (extended test matrix)

This average indicates that for most gait speeds, maximum leg angle is expected to be greater in physical testing than in the gait models employed, with this difference most pronounced at lower speeds. 


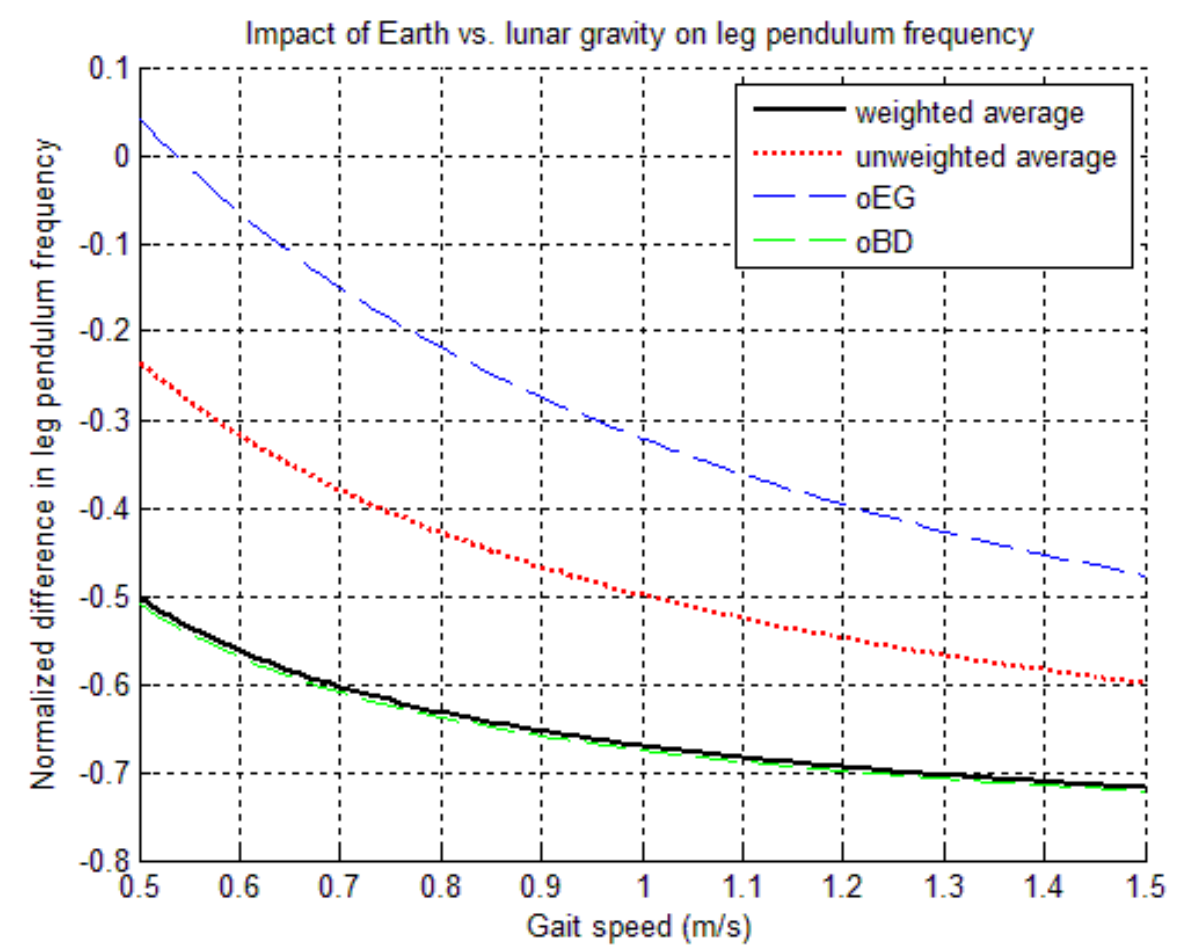

Figure 43: gravity impact on swing frequency (extended test matrix)

This weighted average indicates that over the range of speeds condsidered, leg pendulum frequency is expected to be greater in Earth gravity than in lunar gravity, with the effect being larger at greater speeds. This agrees with intuition that the frequency of an unforced pendulum is greater in higher gravity environments. 


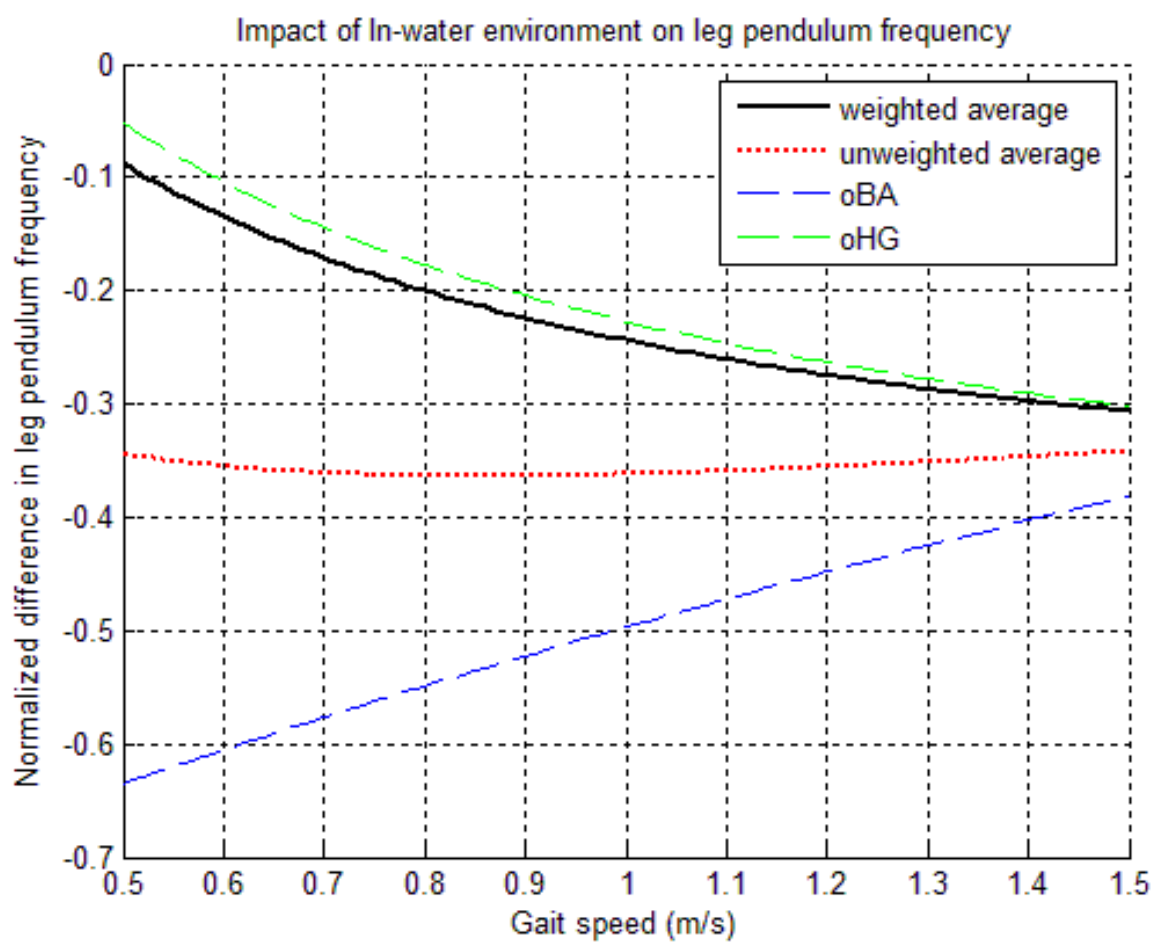

Figure 44: In-water impact on swing frequency (extended test matrix)

This weighted average indicates that over the range of speeds considered, pendulum frequencies are expected to be greater in underwater environments than drag-free environments. 


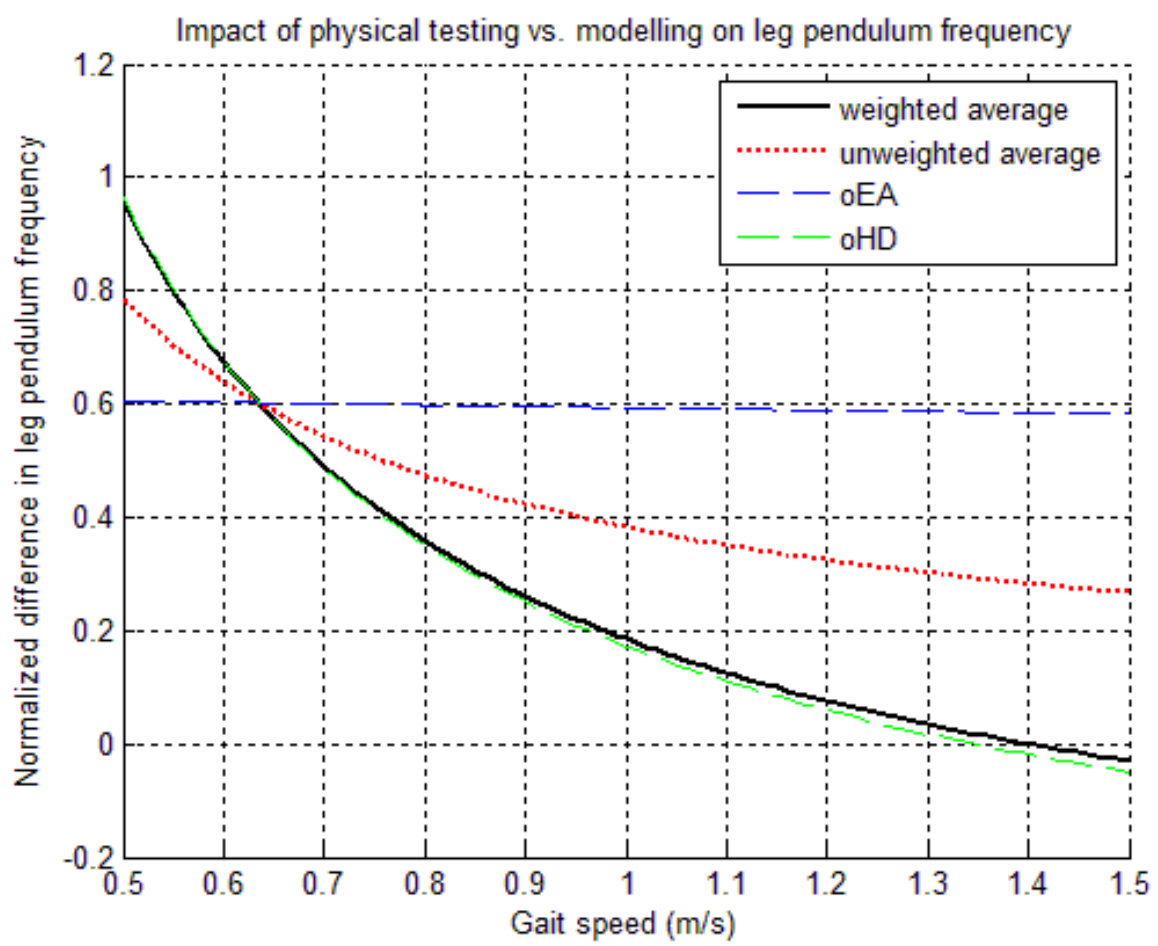

Figure 45: Modelling impact on swing frequency (extended test matrix)

This weighted average indicates that for most gait speeds, pendulum frequencies are expected to be greater in physical testing than in the employed gait models. 
Appendix 4: Extended test matrix - model data

Earth gravity:

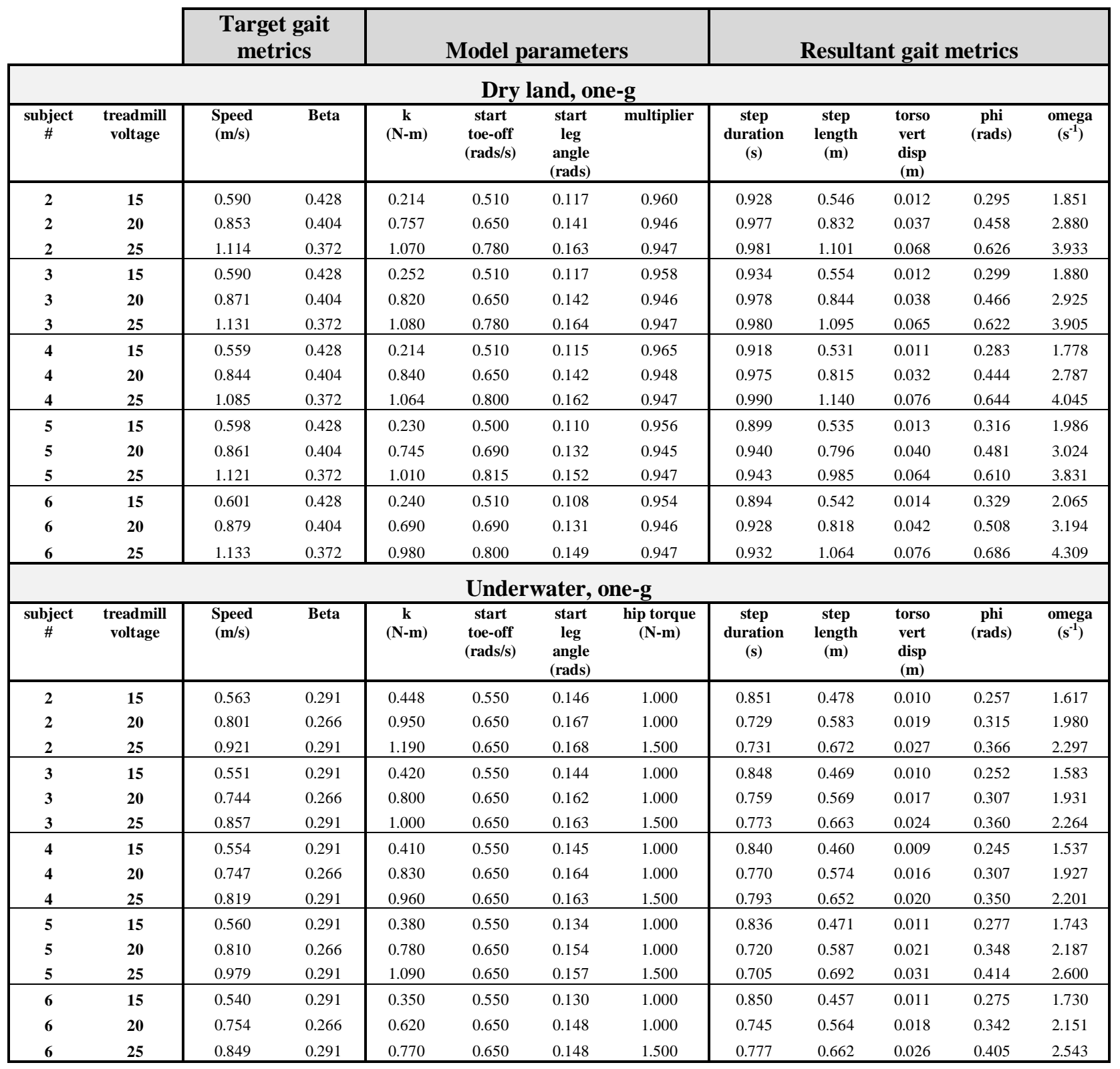




\section{Lunar gravity:}

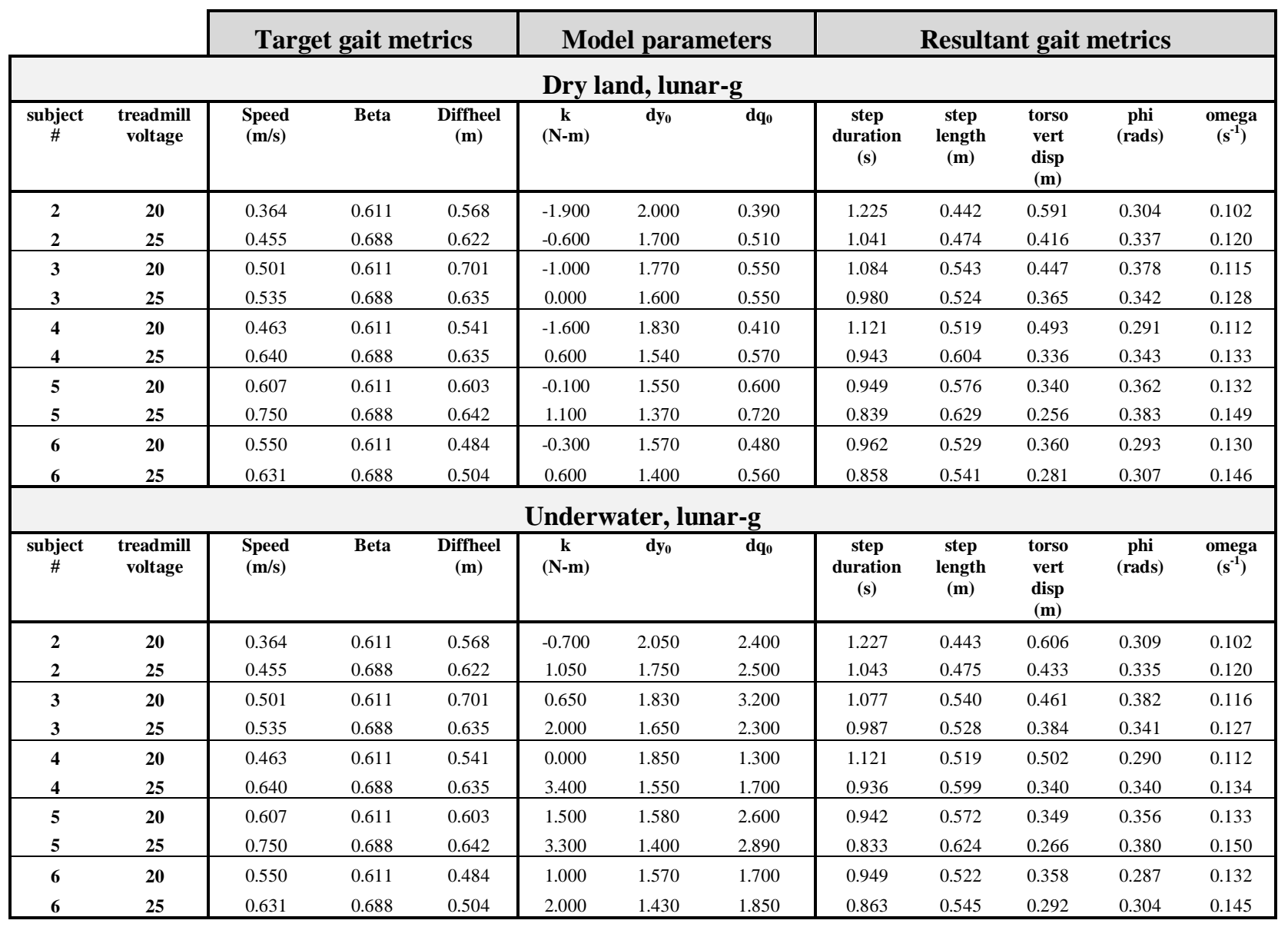




\section{Appendix 5: Matlab code}

\section{Appendix 5a: analyses.m}

function metrics = dry_analyses(datset, secs, knee_height, hip_height, plots, dry, strobe)

$\%$ Cleans up and analyzes a run

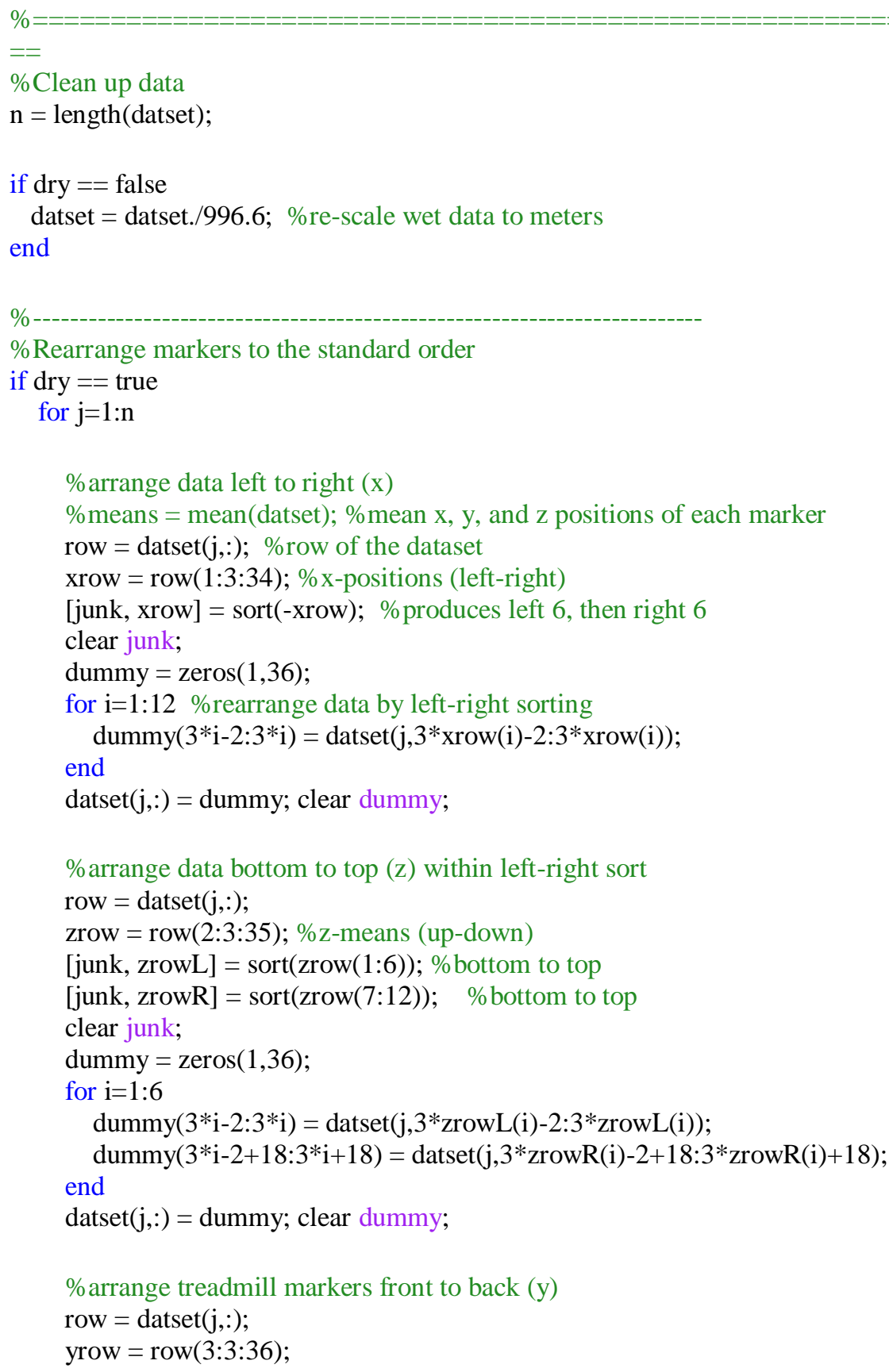




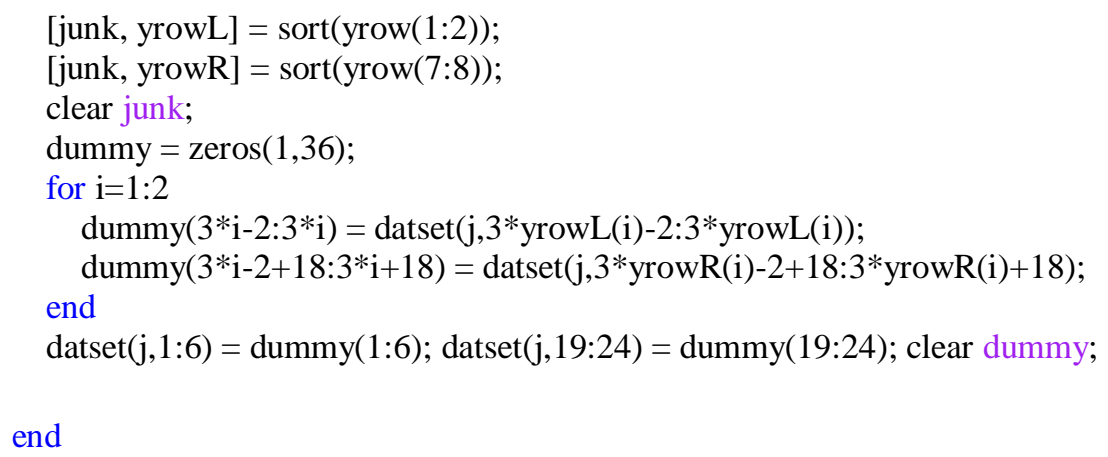

Lfrontx $=$ datset(:,1); Lfronty $=$ datset(:,2); Lfrontz $=$ datset(:,3);

Rfrontx $=$ datset(:,4); Rfronty $=$ datset(:,5); Rfrontz $=$ datset(:,6);

Lrearx $=\operatorname{datset}(:, 7) ; \quad$ Lreary $=\operatorname{datset}(:, 8) ; \quad$ Lrearz $=$ datset(:,9);

Rrearx $=$ datset(:,10); Rreary $=\operatorname{datset}(:, 11) ;$ Rrearz $=\operatorname{datset}(:, 12)$;

Lanklex = datset(:,13); Lankley $=$ datset(:,14); Lanklez $=\operatorname{datset}(:, 15)$;

Ranklex = datset(:,16); Rankley $=$ datset(:,17); Ranklez $=$ datset(:,18);

Lkneex $=$ datset(:,19); Lkneey $=$ datset(:,20); Lkneez $=$ datset(:,21);

Rkneex $=$ datset(:,22); Rkneey $=$ datset(:,23); Rkneez $=$ datset $(:, 24)$;

Lthighx $=$ datset(:,25); Lthighy $=$ datset(:,26); Lthighz $=$ datset(:,27);

Rthighx $=$ datset(:,28); Rthighy $=\operatorname{datset}(:, 29) ;$ Rthighz $=\operatorname{datset}(:, 30)$;

Lhipx = datset(:,31); Lhipy = datset(:,32); Lhipz = datset(:,33);

Rhipx $=\operatorname{datset}(:, 34) ; \quad$ Rhipy $=\operatorname{datset}(:, 35) ; \quad$ Rhipz $=\operatorname{datset}(:, 36)$;

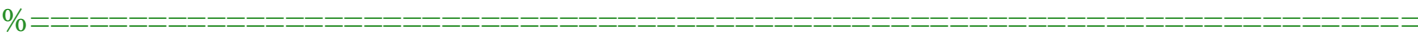

$==$

\%Create virtual knee, heel, and torso markers from known geometry

$\%$ Torso

torsox = mean $([$ Lhipx Rhipx $], 2)$;

torsoy $=$ mean ([Lhipy Rhipy $], 2)$;

torsoz $=$ mean $([$ Lhipz Rhipz $], 2)$; 


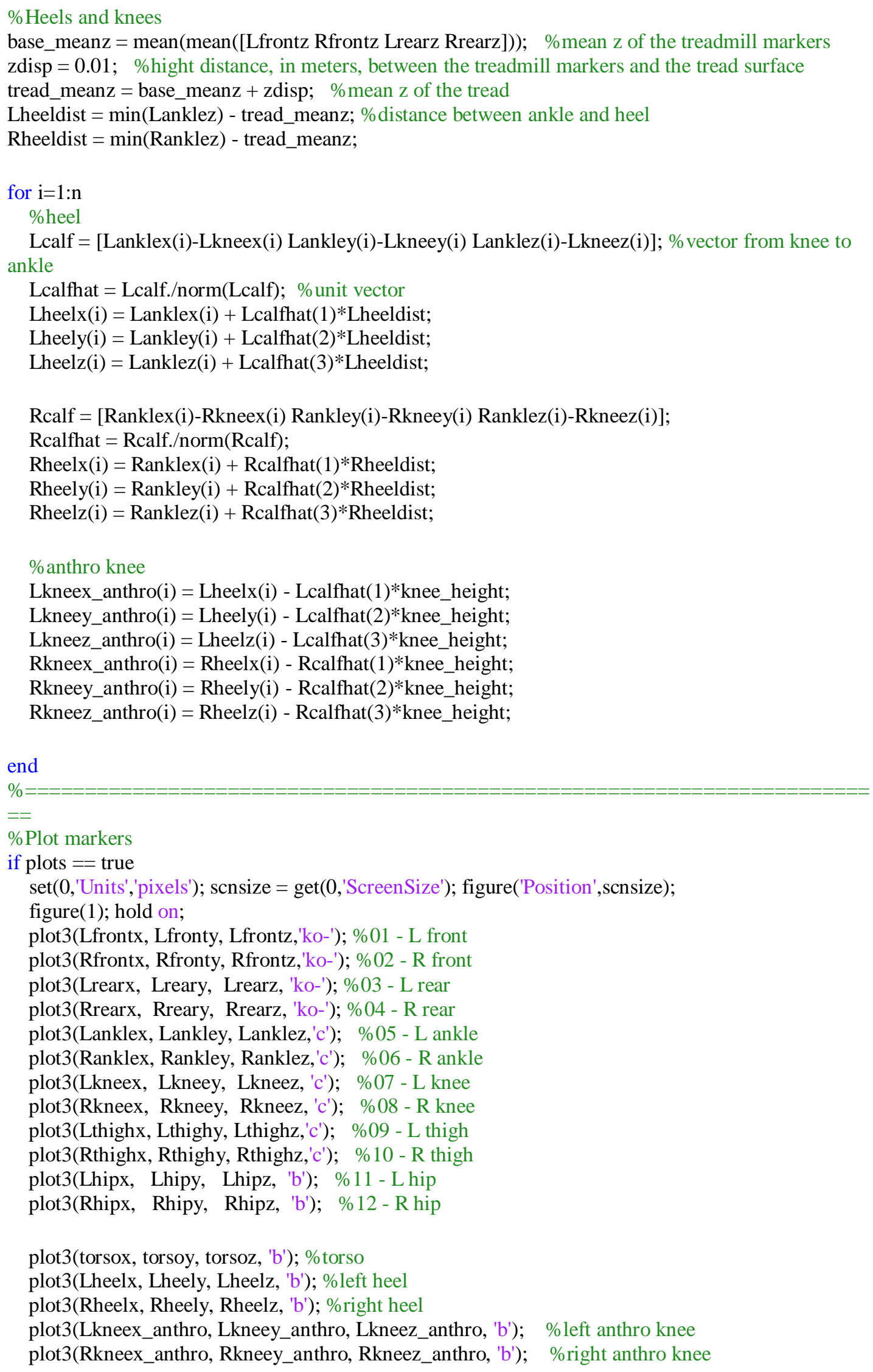


grid on; axis square; axis equal; xlabel('x'); ylabel('y'); zlabel('z'); view(160,10);

end

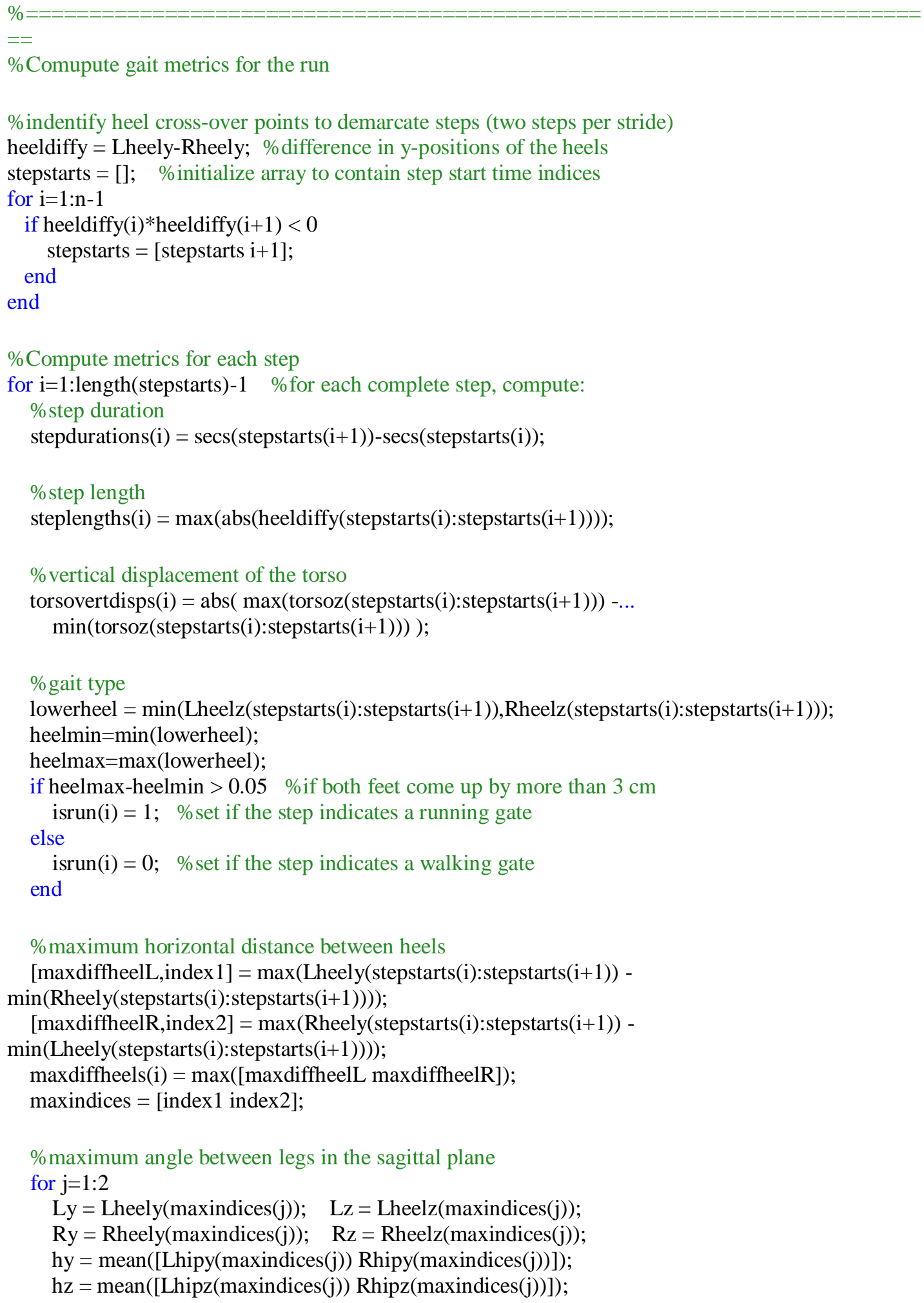




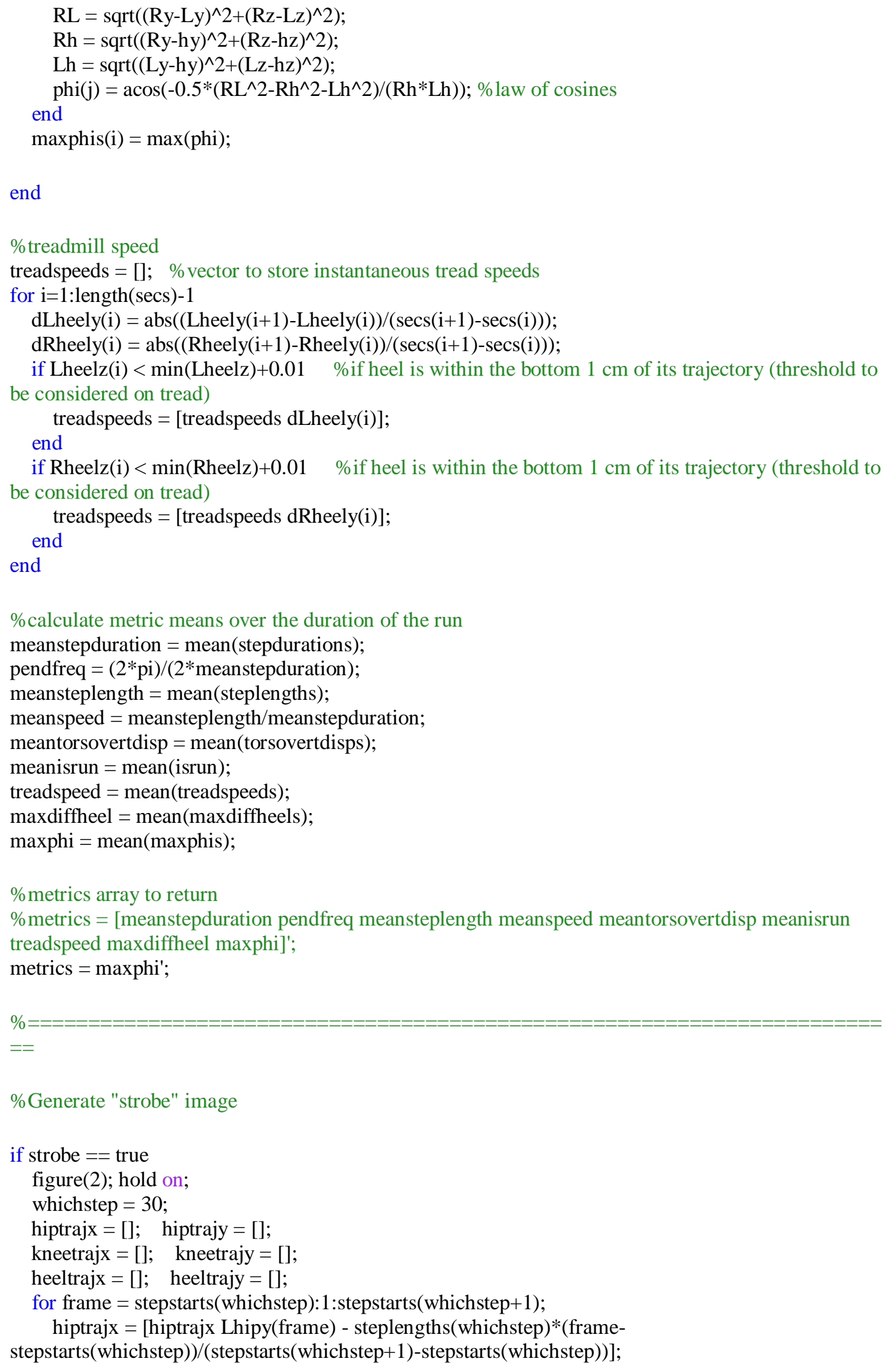




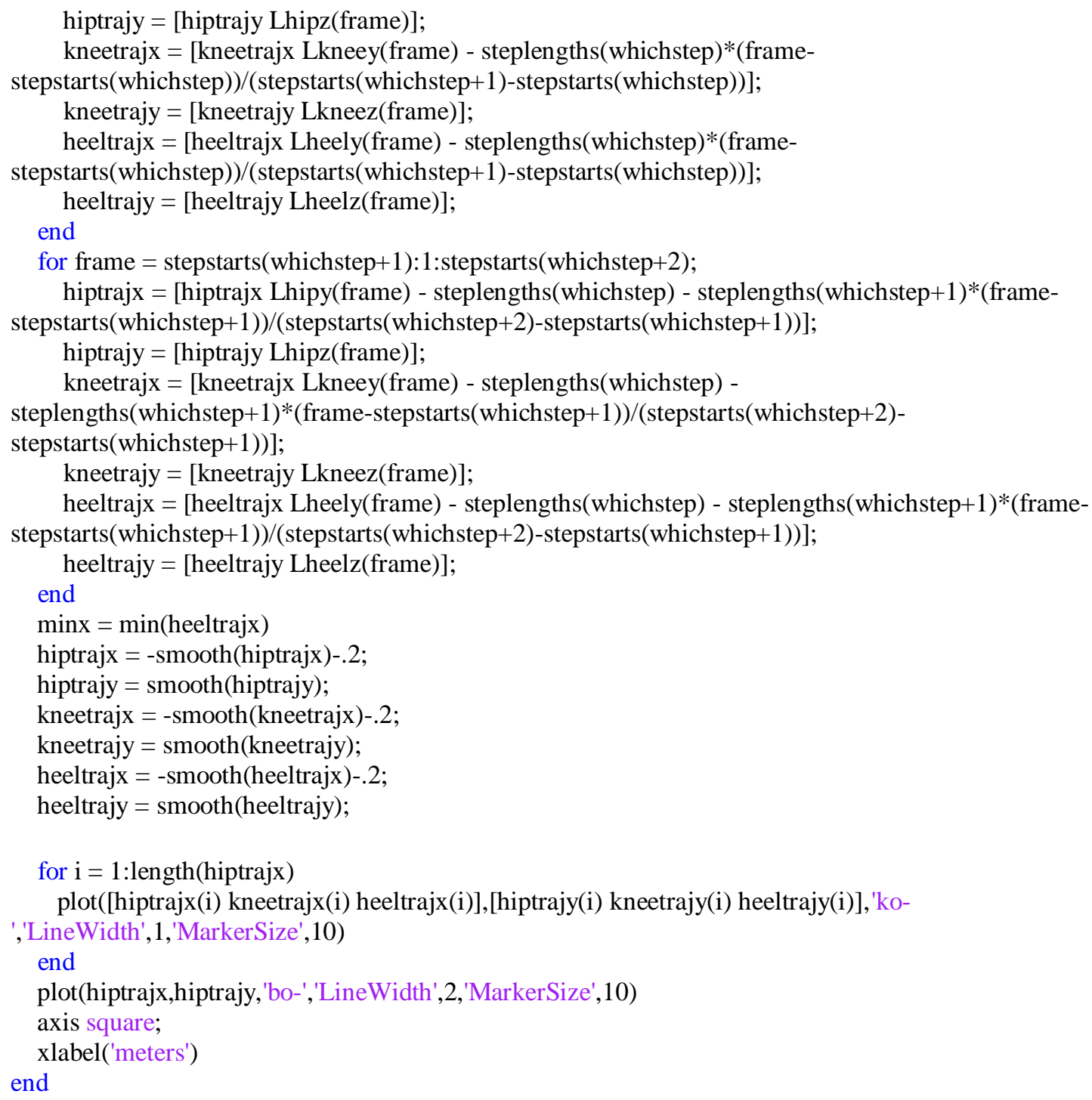




\section{Appendix 5b: container.m}

$\%$ Get data, get it cleaned up and analyzed

clear all; close all; clc;

\%subj2 (one-g dry, one-g wet, lunar wet)

[datset secs knee_height hip_height] = dry15_subj2();

subj2_metrics $(:, 1)=$ analyses(datset, secs, knee_height, hip_height, 0, 1, 0);

[datset secs knee_height hip_height] = dry20_subj2();

subj2_metrics $(:, 2)=$ analyses(datset, secs, knee_height, hip_height, 0, 1, 0);

[datset secs knee_height hip_height] = dry25_subj2();

subj2_metrics $(:, 3)=$ analyses(datset, secs, knee_height, hip_height, 0, 1, 0);

[datset secs knee_height hip_height] = oneg15_subj2();

subj2_metrics $(:, 4)=$ analyses(datset, secs, knee_height, hip_height, 0, 0, 0); [datset secs knee_height hip_height] = oneg20_subj2( $)$;

subj2_metrics $(:, 5)=$ analyses(datset, secs, knee_height, hip_height, 0, 0, 0); [datset secs knee_height hip_height] = oneg25_subj2();

subj2_metrics $(:, \overline{6})=$ analyses(datset, secs, knee_height, hip_height, 0, 0, 0);

[datset secs knee_height hip_height] = lunar15_subj2();

subj2_metrics $(:, 7)=$ analyses(datset, secs, knee_height, hip_height, $0,0,0$ ); [datset secs knee_height hip_height] = lunar20_subj2();

subj2_metrics $(:, 8)=$ analyses(datset, secs, knee_height, hip_height, 0, 0, 0); [datset secs knee_height hip_height] = lunar25_subj2();

subj2_metrics(:,9) = analyses(datset, secs, knee_height, hip_height, 0, 0,0);

$\%$ subj3 (one-g dry, one-g wet, lunar wet)

[datset secs knee_height hip_height] = dry15_subj3();

subj3_metrics $(:, \overline{1})=$ analyses(datset, secs, knee_height, hip_height, $0,1,0)$;

[datset secs knee_height hip_height] = dry20_subj3();

subj3_metrics $(:, 2)=$ analyses(datset, secs, knee_height, hip_height, 0, 1, 0);

[datset secs knee_height hip_height] = dry25_subj3();

subj3_metrics $(:, 3)=$ analyses(datset, secs, knee_height, hip_height, 0, 1, 0);

[datset secs knee_height hip_height] = oneg15_subj3( );

subj3_metrics $(:, 4)=$ analyses(datset(:,1:36), secs, knee_height, hip_height, 0, 0, 0);

[datset secs knee_height hip_height] = oneg20_subj3();

subj3_metrics $(: \overline{5})=$ analyses $(\operatorname{datset}(20: 1109,1: 36), \operatorname{secs}(20: 1109)$, knee_height, hip_height, 0, 0, 0);

[datset secs knee_height hip_height] = oneg25_subj3();

subj3_metrics $(:, 6)=$ analyses(datset(:,1:36), secs, knee_height, hip_height, 0, 0, 0);

[datset secs knee_height hip_height] = lunar15_subj3();

subj3_metrics(:,7) = analyses(datset(:,1:36), secs, knee_height, hip_height, 0, 0, 0);

[datset secs knee_height hip_height] = lunar20_subj3();

subj3_metrics $(:, 8)=$ analyses(datset(:,1:36), secs, knee_height, hip_height, 0, 0, 0);

[datset secs knee_height hip_height] = lunar25_subj3();

subj3_metrics(:,9) = analyses(datset(:,1:36), secs, knee_height, hip_height, 0,0,0);

\%subj4 (one-g dry, one-g wet, lunar wet)

[datset secs knee_height hip_height] = dry15_subj4(); 
subj4_metrics1 $=$ analyses(datset(1:769,:), secs(1:769), knee_height, hip_height, $0,1,0)$;

subj4_metrics2 = analyses(datset(772:1059,:), secs(772:1059), knee_height, hip_height, 0, 1, 0); subj4_metrics(:,1) $=(769 *$ subj4_metrics1 + 288*subj4_metrics2)./1057;

[datset secs knee_height hip_height] = dry20_subj4();

subj4_metrics3 = analyses(datset(1:449,:), secs(1:449), knee_height,hip_height, $0,1,0)$;

subj4_metrics4 = analyses(datset(451:890,:), secs(451:890), knee_height, hip_height, 0, 1, 0);

subj4_metrics5 = analyses(datset(892:1023,:), $\operatorname{secs}(892: 1023)$, knee_height, hip_height, 0, 1, 0);

subj4_metrics $(:, 2)=\left(449 * \operatorname{subj} 4 \_m e t r i c s 3+440 * \operatorname{subj} 4 \_m e t r i c s 4+132 * s u b j 4 \_m e t r i c s 5\right) . / 1021$;

[datset secs knee_height hip_height] = dry25_subj4();

subj4_metrics(:,3) = analyses(datset, secs, knee_height, hip_height, $0,1,0)$;

[datset secs knee_height hip_height] = oneg15_subj4();

subj4_metrics(:,4) = analyses (datset, secs, knee_height, hip_height, 0, 0, 0);

[datset secs knee_height hip_height] = oneg20_subj4();

subj4_metrics(:,5) = analyses (datset, secs, knee_height, hip_height, 0, 0, 0);

[datset secs knee_height hip_height] = oneg25_subj4();

subj4_metrics $(:, 6)=$ analyses(datset, secs, knee_height, hip_height, 0, 0, 0);

[datset secs knee_height hip_height] = lunar15_subj4();

subj4_metrics $(:, 7)=$ analyses (datset, secs, knee_height, hip_height, $0,0,0$ );

[datset secs knee_height hip_height] = lunar20_subj4();

subj4_metrics(:,8) = analyses(datset, secs, knee_height, hip_height, 0, 0, 0);

[datset secs knee_height hip_height] = lunar25_subj4();

subj4_metrics(:,9) = analyses(datset, secs, knee_height, hip_height, 0, 0, 0);

$\%$ subj5 (one-g dry, one-g wet, lunar wet)

[datset secs knee_height hip_height] = dry15_subj5();

subj5_metrics(:,1) = analyses(datset, secs, knee_height, hip_height, $0,1,0$ );

[datset secs knee_height hip_height] = dry20_subj5();

subj5_metrics(:,2) = analyses(datset, secs, knee_height, hip_height, 0, 1, 0);

[datset secs knee_height hip_height] = dry25_subj5();

subj5_metrics(:,3) = analyses(datset, secs, knee_height, hip_height, 0, 1, 0);

[datset secs knee_height hip_height] = oneg15_subj5();

subj5_metrics(:,4) = analyses (datset, secs, knee_height, hip_height, 0, 0, 0); [datset secs knee_height hip_height] = oneg20_subj5();

subj5_metrics(:,5) = analyses(datset, secs, knee_height, hip_height, 0, 0, 0); [datset secs knee_height hip_height] = oneg25_subj5();

subj5_metrics(:,6) = analyses(datset, secs, knee_height, hip_height, 0, 0, 0);

[datset secs knee_height hip_height] = lunar15_subj5();

subj5_metrics(:,7) = analyses(datset, secs, knee_height, hip_height, 0, 0, 0); [datset secs knee_height hip_height] = lunar20_subj5();

subj5_metrics(:(8) = analyses(datset, secs, knee_height, hip_height, 0, 0, 0); [datset secs knee_height hip_height] = lunar25_subj5();

subj5_metrics(:,9) = analyses(datset, secs, knee_height, hip_height, $0,0,0)$;

\%subj6 (one-g dry, one-g wet, lunar wet)

[datset secs knee_height hip_height] = dry15_subj6();

subj6_metrics(:,1) = analyses(datset, secs, knee_height, hip_height, 0, 1, 0); [datset secs knee_height hip_height] = dry20_subj6();

subj6_metrics(:,2) = analyses(datset, secs, knee_height, hip_height, 0, 1, 0); [datset secs knee_height hip_height] = dry25_subj6();

subj6_metrics(:,3) = analyses(datset, secs, knee_height, hip_height, $0,1,0)$; 
[datset secs knee_height hip_height] = oneg15_subj6();

subj6_metrics(:,4) = analyses (datset, secs, knee_height, hip_height, 0, 0, 0);

[datset secs knee_height hip_height] = oneg20_subj6();

subj6_metrics(:,5) = analyses(datset, secs, knee_height, hip_height, 0, 0, 0);

[datset secs knee_height hip_height] = oneg25_subj6();

subj6_metrics1 = analyses(datset(1:229,:), secs(1:229), knee_height, hip_height, 0, 0, 0);

subj6_metrics2 $=$ analyses (datset(260:419,:), secs(260:419),knee_height, hip_height, 0, 0, 0);

subj6_metrics3 = analyses(datset(430:497,:), secs(430:497), knee_height, hip_height, 0, 0, 0);

subj6_metrics(:,6) = (229*subj6_metrics1 + 160*subj6_metrics2 + 68*subj6_metrics3)./497;

[datset secs knee_height hip_height] = lunar15_subj6();

subj6_metrics(:,7) = analyses(datset, secs, knee_height, hip_height, 0, 0, 0);

[datset secs knee_height hip_height] = lunar20_subj6();

subj6_metrics(:,8) = analyses (datset, secs, knee_height, hip_height, 0, 0, 0);

[datset secs knee_height hip_height] = lunar25_subj6();

subj6_metrics(:,9) = analyses(datset, secs, knee_height, hip_height, 0, 0, 0);

subj2_metrics

subj3_metrics

subj4_metrics

subj5_metrics

subj6_metrics

[subj2_metrics;subj3_metrics;subj4_metrics;subj5_metrics;subj6_metrics] 


\section{Appendix 5c: simplerunnerEOM.m}

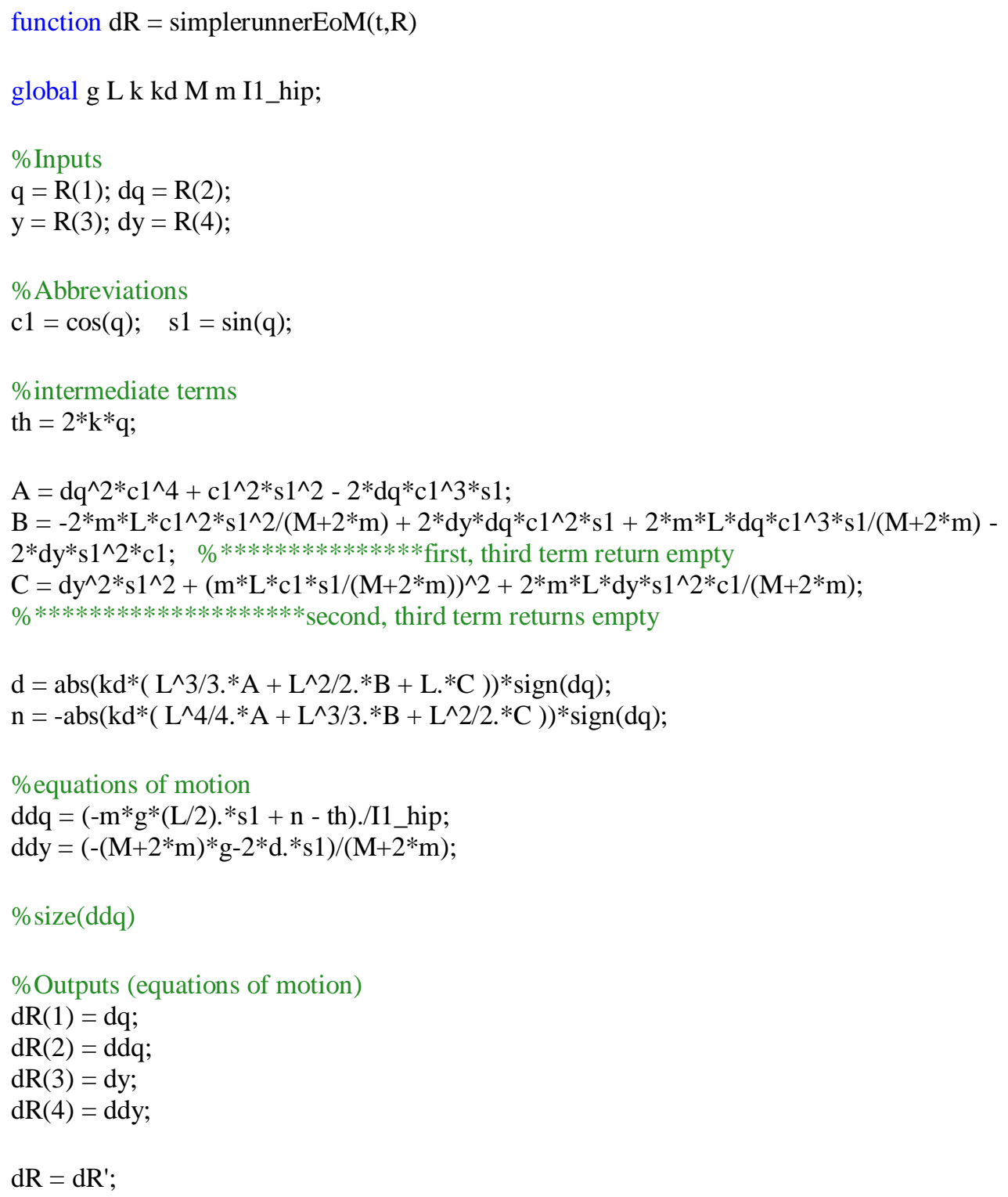




\section{Appendix 5d: simplerunner_sim.m}

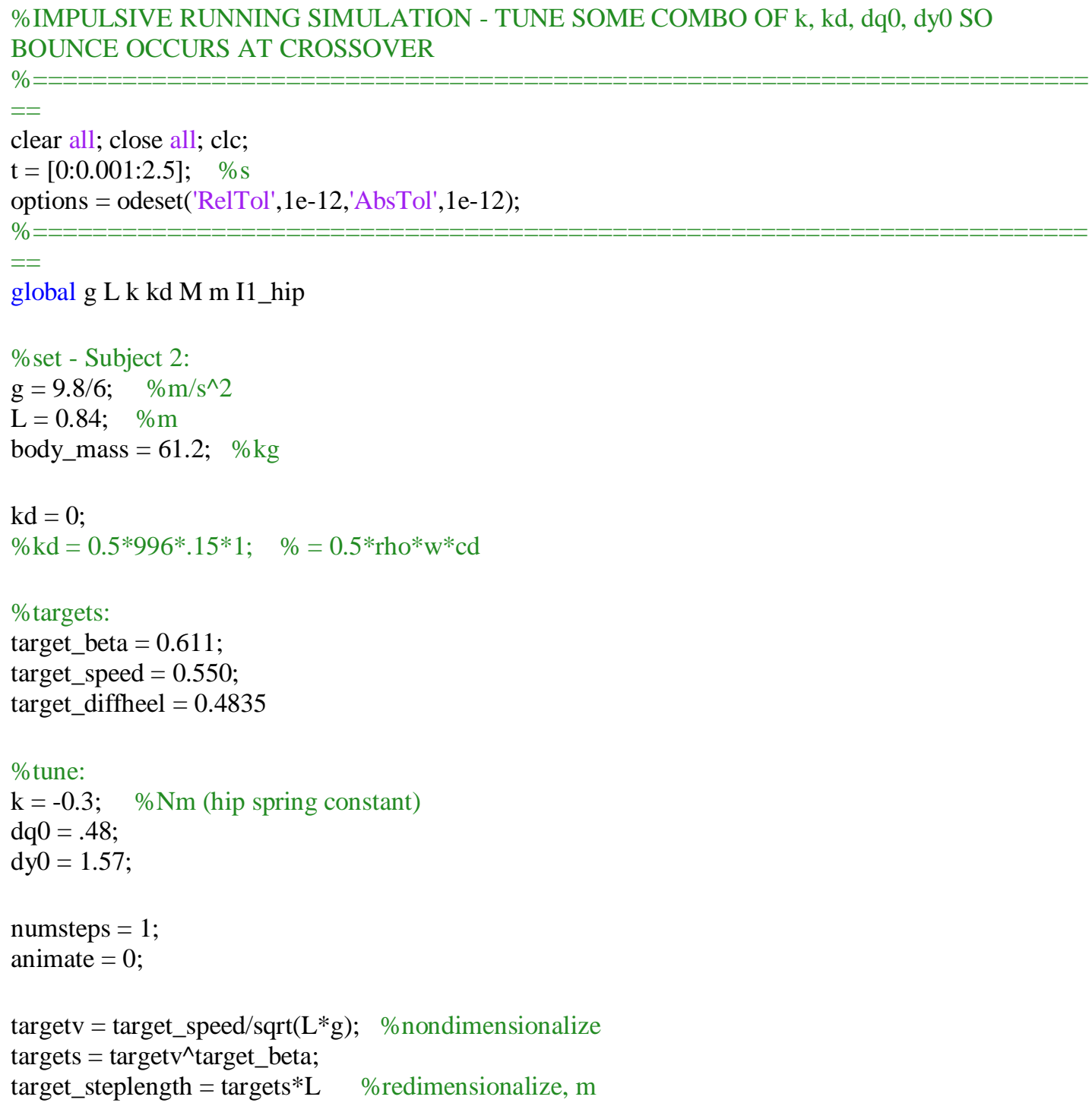

[k kd dy0 dq0]'

$\%$ Working values

$\% \mathrm{M}=51.4 ; \% \mathrm{~kg}$ (torso)

$\% \mathrm{~m}=15.4 ; \% \mathrm{~kg}$ (one leg)

$\mathrm{M}=0.62 *$ body_mass;

$\mathrm{m}=0.19 *$ body_mass;

I1_hip $=m * L^{\wedge} 2 / 3$;

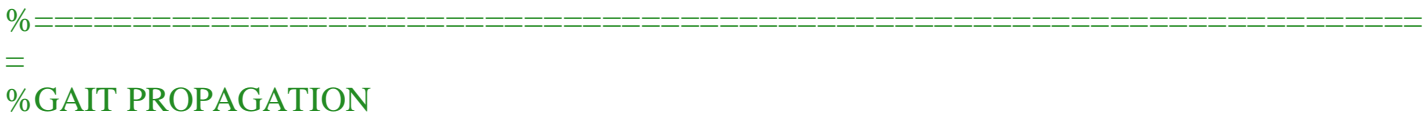




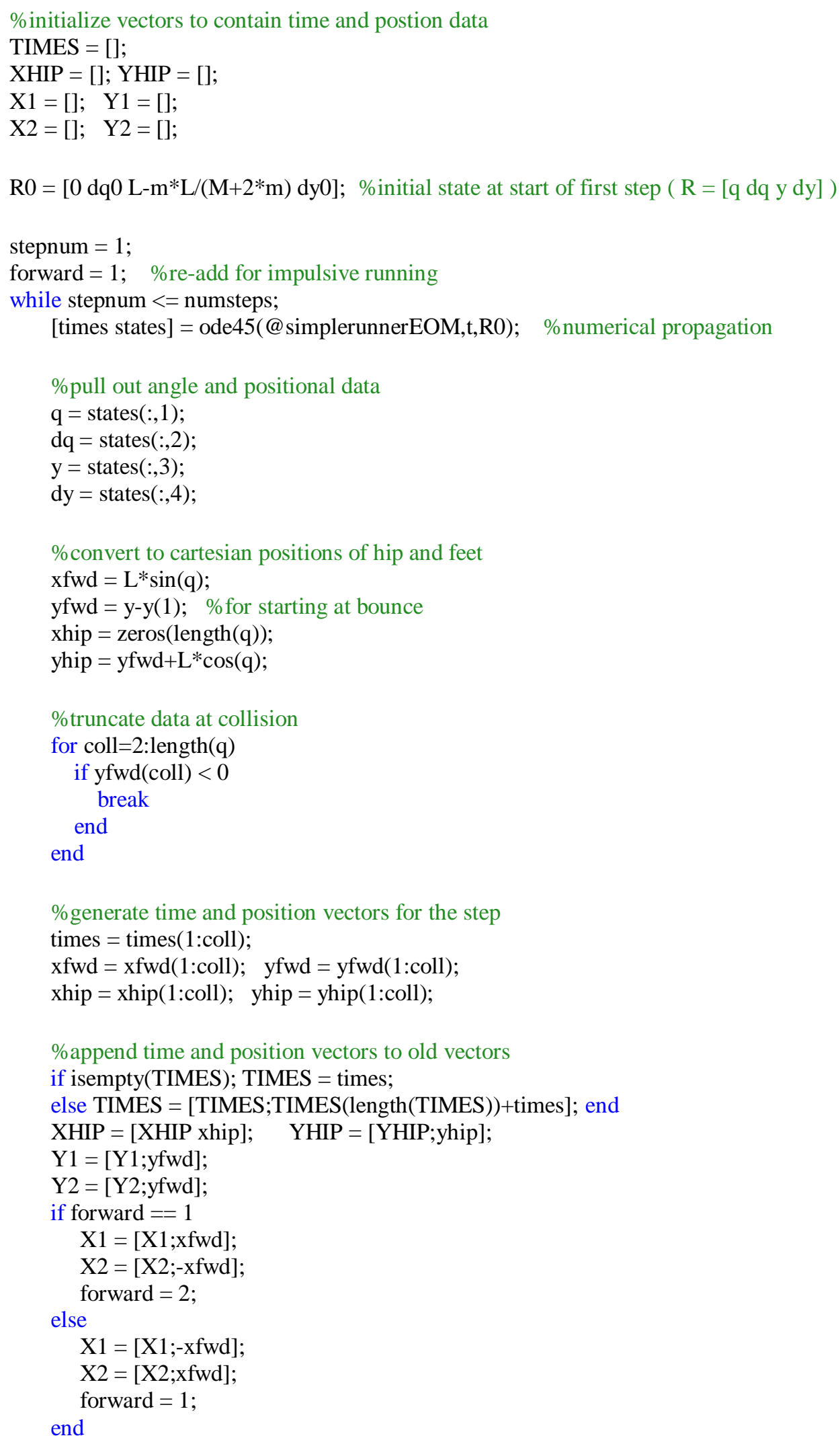




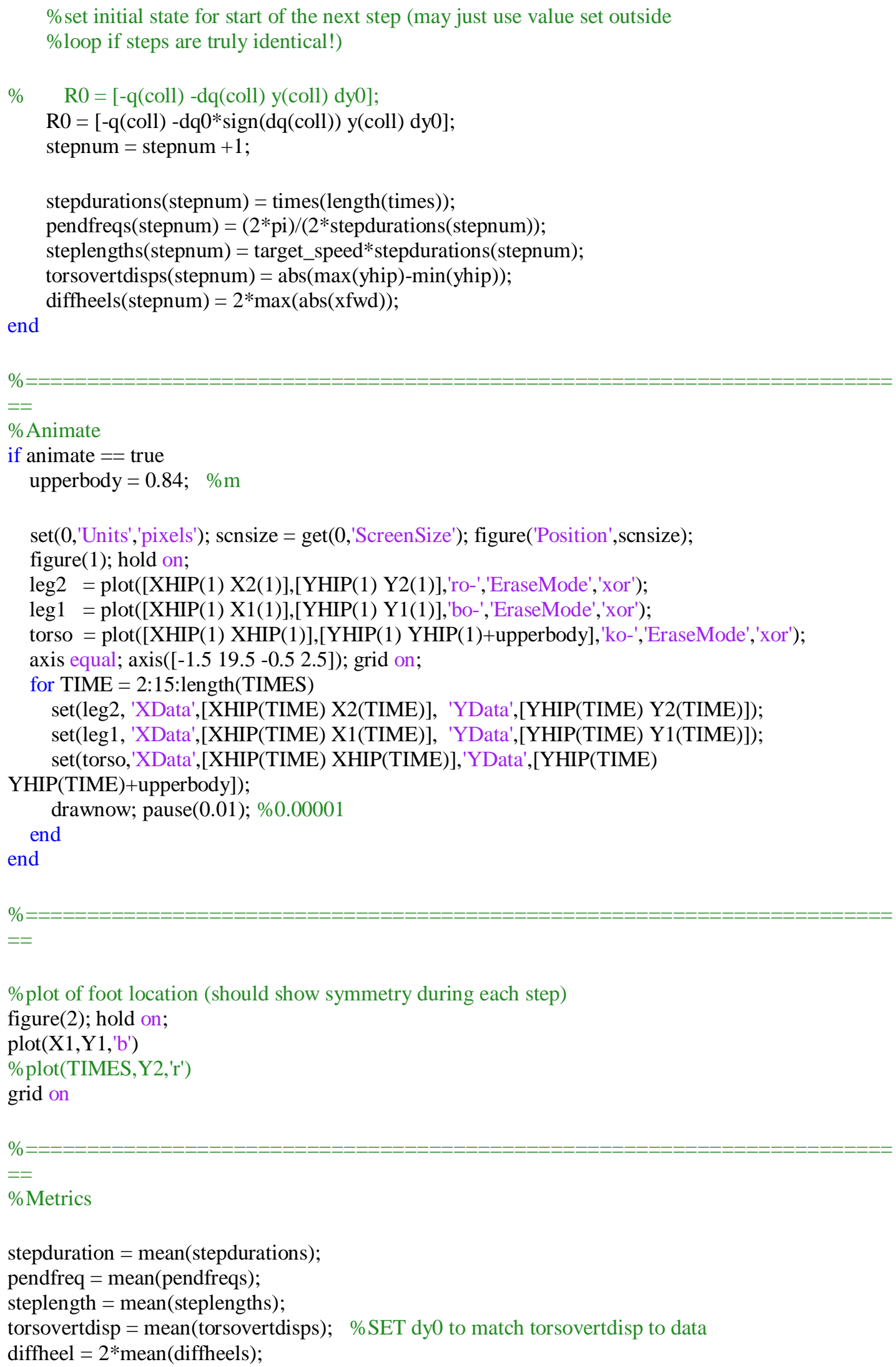


[stepduration pendfreq steplength torsovertdisp diffheel]'

diffheel

steplength

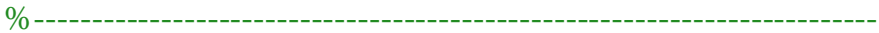

$\%$ END OF CODE 


\section{Appendix 5e: gaitEOMnew.m}

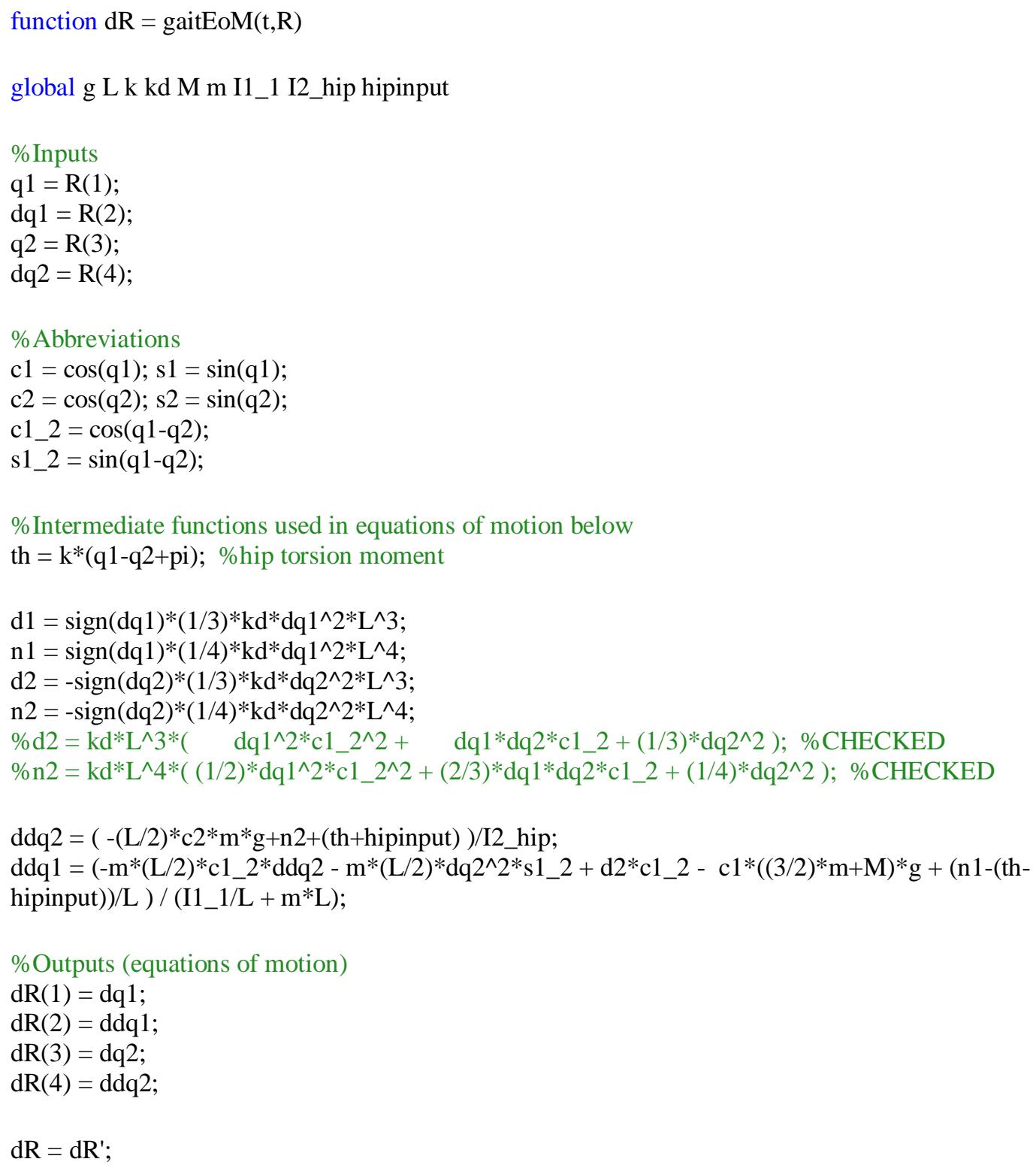




\section{Appendix 5f: gait_sim.m}

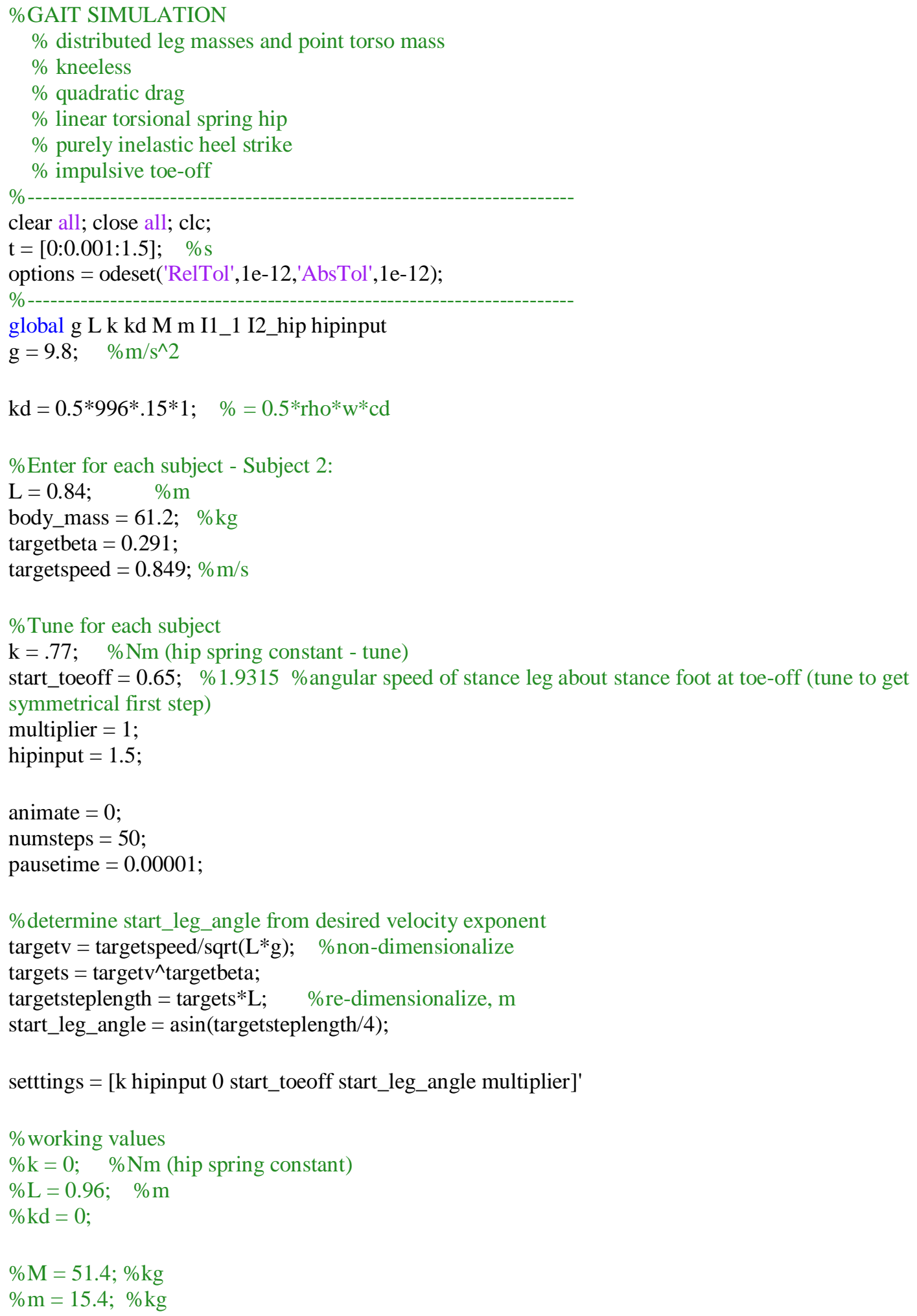


\%start_leg_angle $=0.11 *$ pi; \%initial maximum angle of legs from vertical

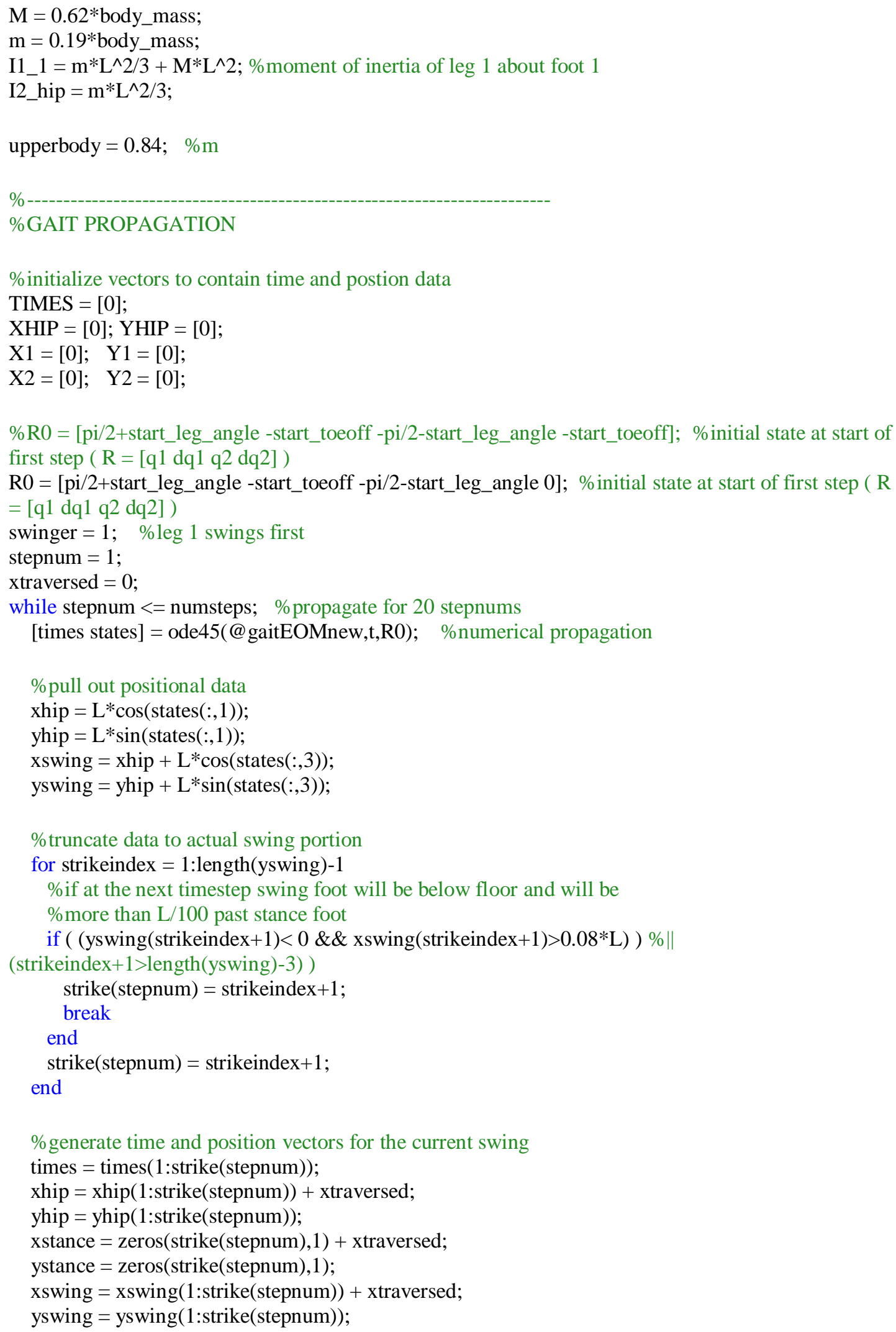




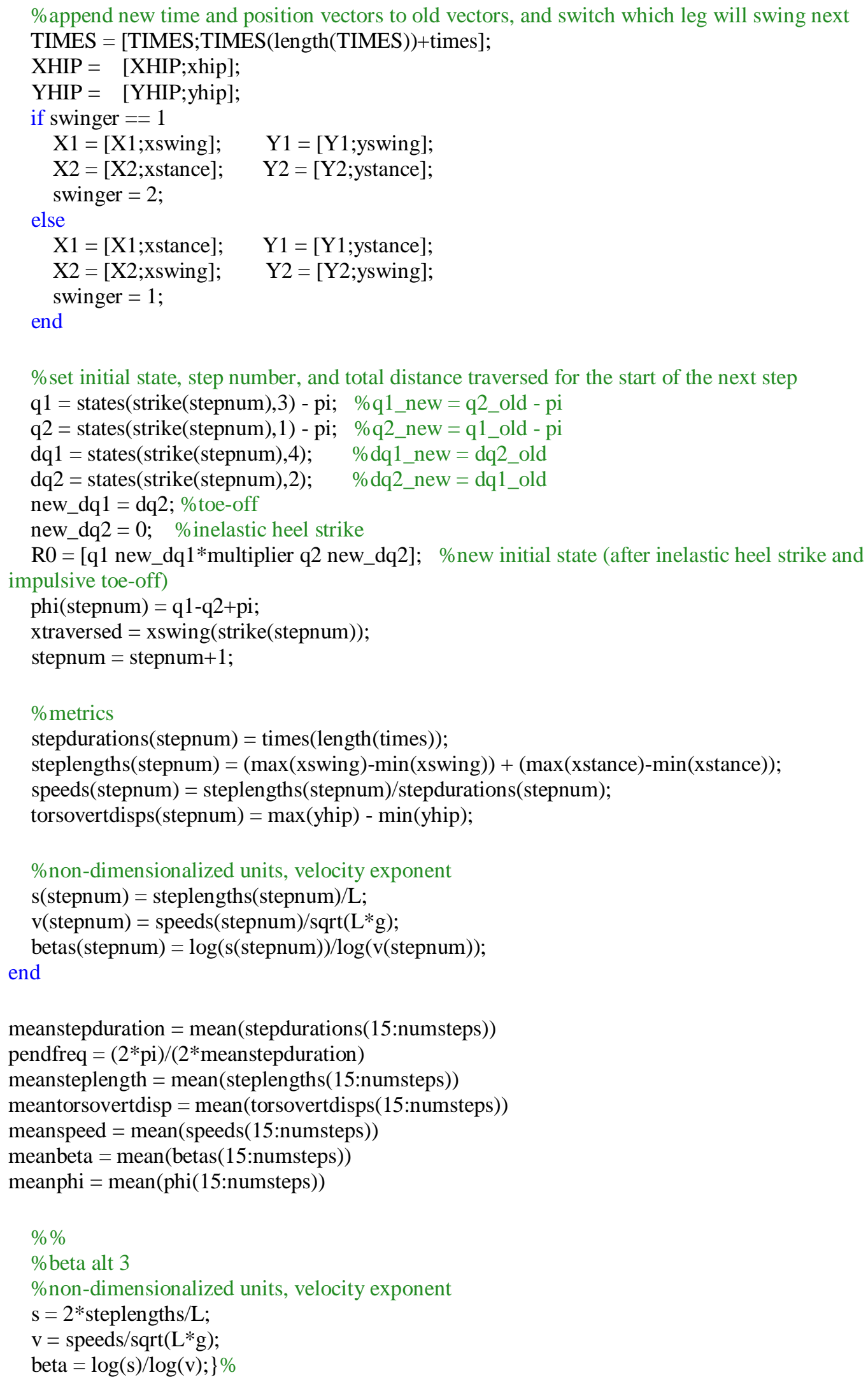


[meanstepduration pendfreq meansteplength meantorsovertdisp meanspeed meanbeta meanphi]'

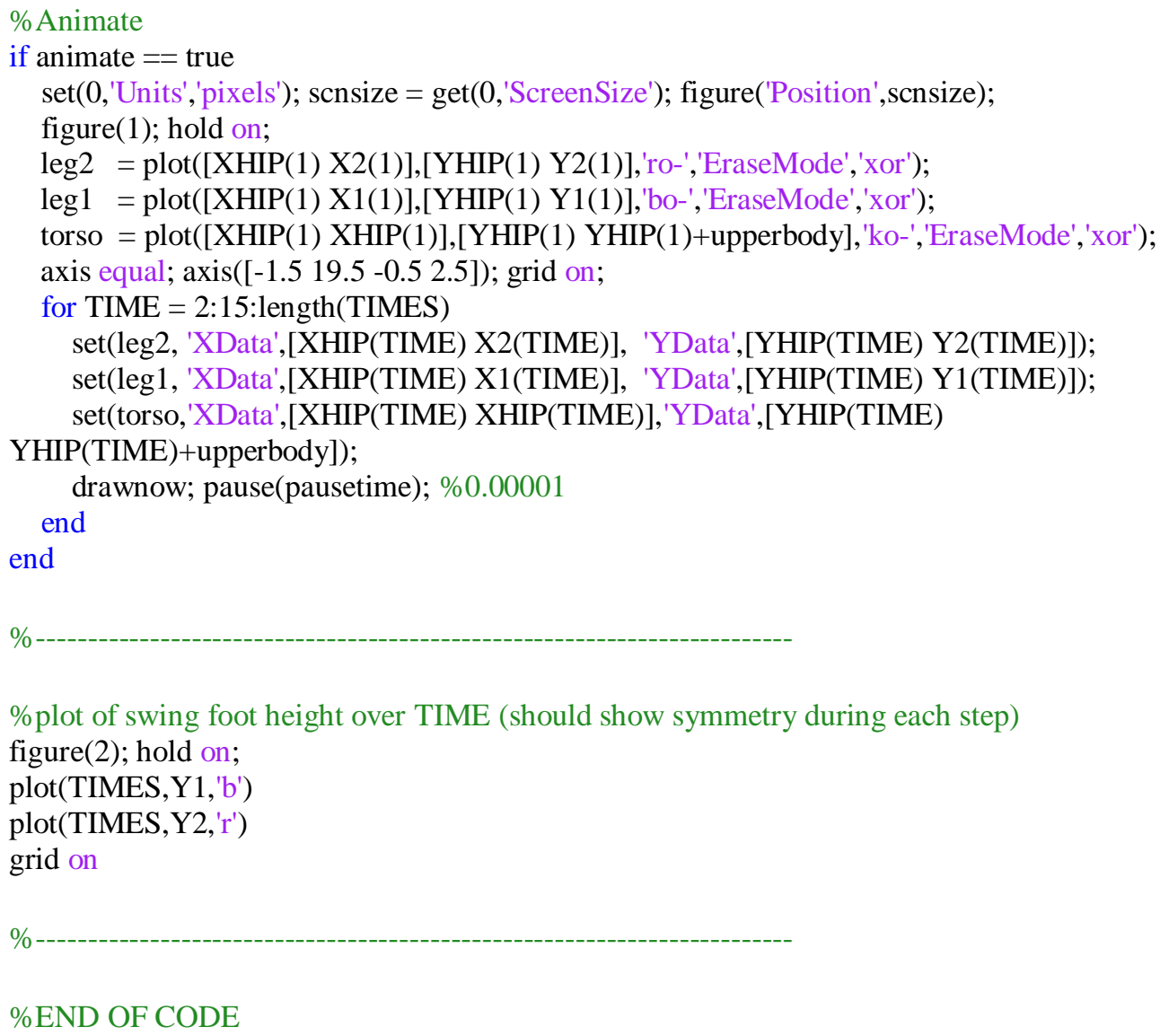


Appendix 5g: goal1.m

clear all; close all; clc;

$\%$ Estimate gait metrics as a function of speed for true lunar gravity

$$
\begin{aligned}
& \mathrm{x}=(0: 0.01: 3) ; \% \text { speed }, \mathrm{m} / \mathrm{s} \\
& \% \text { Metric-estimating functions } \\
& \% \text { Step length vs. gait speed } \\
& \mathrm{sH}=0.3275 . * \mathrm{x}+0.2651 ; \mathrm{RsH}=0.6782 ; \\
& \mathrm{sG}=0.2826 * \mathrm{x}+0.3995 ; \mathrm{RsG}=0.5285 \text {; } \\
& \mathrm{sE}=0.2766 . * \mathrm{x}+0.3846 ; \mathrm{RsE}=0.2503 ; \\
& \mathrm{sD}=1.0149 \cdot{ }^{*} \mathrm{x}-0.0566 ; \mathrm{RsD}=0.9918 ; \\
& \mathrm{sB}=0.4647 . * \mathrm{x}+0.2828 ; \mathrm{RsB}=0.8675 \text {; } \\
& \mathrm{sA}=0.4514 . * \mathrm{x}+0.2886 ; \mathrm{RsA}=0.8796 \\
& \% \text { Vertical torso displacement vs. gait speed } \\
& \mathrm{tH}=0.0319 . * \mathrm{x}+0.0026 ; \mathrm{RtH}=0.6509 ; \\
& \mathrm{tG}=0.0590 . * \mathrm{x}-0.0031 ; \mathrm{RtG}=0.4326 ; \\
& \mathrm{tE}=-0.1858 . * \mathrm{x}+0.1606 ; \mathrm{RtE}=0.5213 \text {; } \\
& \mathrm{tD}=0.1092 . * \mathrm{x}-0.0537 ; \mathrm{RtD}=0.9877 \text {; } \\
& \mathrm{tB}=-0.8430 . * \mathrm{x}+0.8515 ; \mathrm{RtB}=0.8760 ; \\
& \mathrm{tA}=-0.8641 . * \mathrm{x}+0.8736 ; \mathrm{RtA}=0.8836 ; \\
& \% \text { Maximum leg angle vs. gait speed } \\
& \mathrm{pH}=0.3509 . *_{\mathrm{x}}+0.3071 ; \mathrm{RpH}=0.4101 \text {; } \\
& \mathrm{pG}=0.6842 . * \mathrm{x}+0.1673 ; \mathrm{RpG}=0.5452 \text {; } \\
& \mathrm{pE}=0.0942{ }^{*} \mathrm{x}+0.4832 ; \mathrm{RpE}=0.0132 ; \\
& \mathrm{pD}=0.6310 *{ }^{*} \mathrm{x}-0.0697 ; \mathrm{RpD}=0.9870 \text {; } \\
& \mathrm{pB}=0.1283 * \mathrm{x}+0.2619 ; \mathrm{RpB}=0.1754 \text {; } \\
& \mathrm{pA}=0.0644 . * \mathrm{x}+0.2980 ; \mathrm{RpA}=0.0264 \text {; }
\end{aligned}
$$

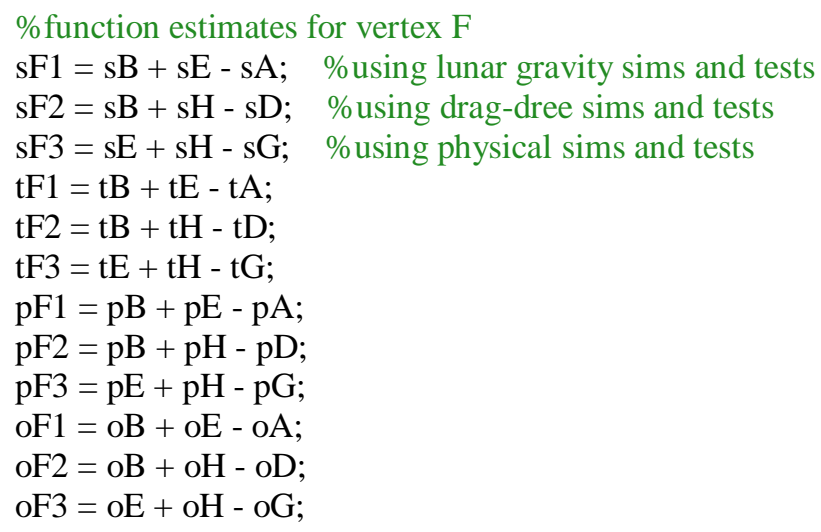




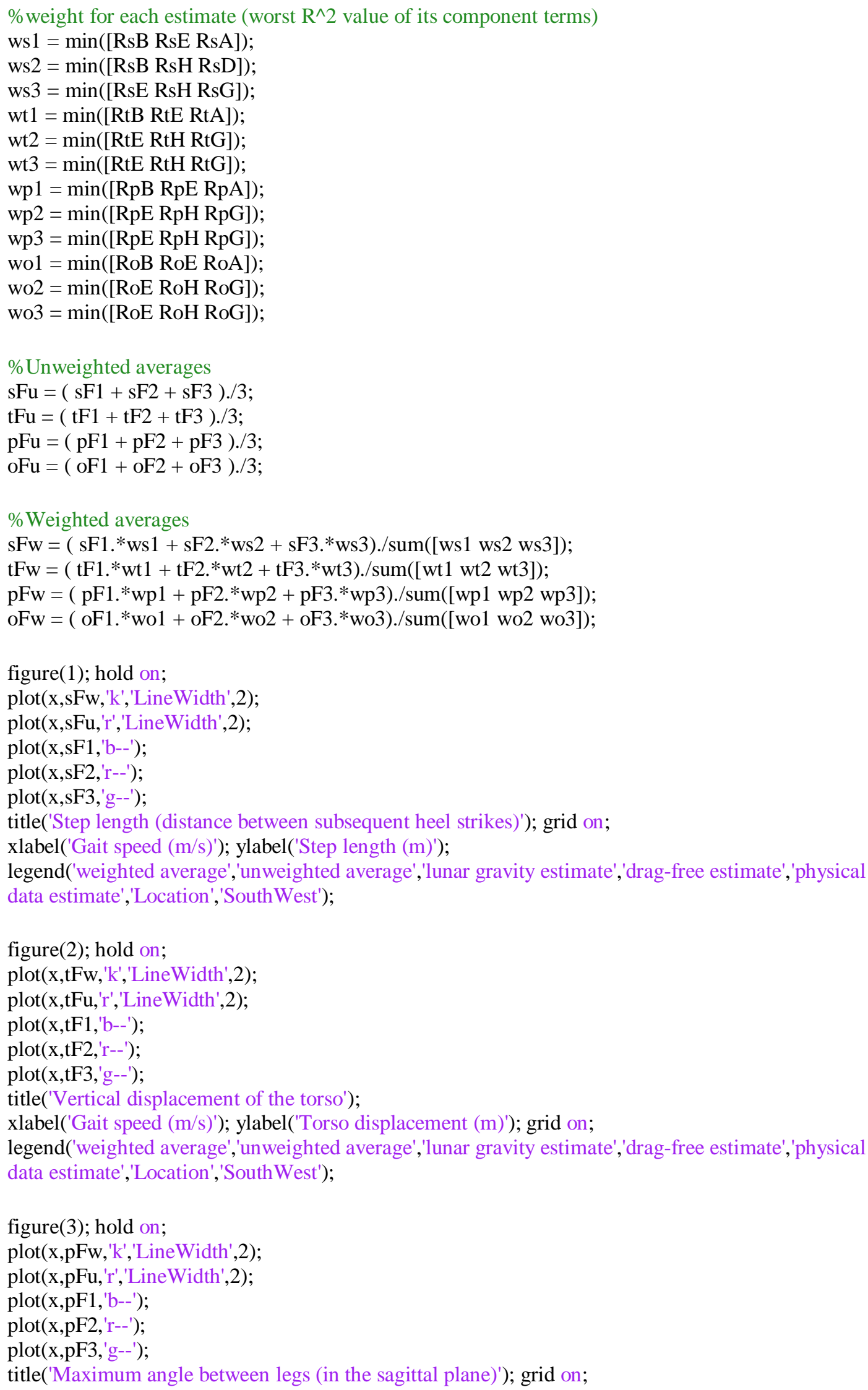


xlabel('Gait speed (m/s)'); ylabel('Leg angle (radians)');

legend('weighted average','unweighted average','lunar gravity estimate','drag-free estimate','physical data estimate','Location','SouthWest');

figure(4); hold on;

plot(x,oFw,'k','LineWidth',2);

plot(x,oFu,'r','LineWidth',2);

$\operatorname{plot}\left(\mathrm{x}, \mathrm{oF} 1, \mathrm{~b}-\mathrm{-}^{\prime}\right)$;

plot $\left(\mathrm{x}, \mathrm{oF} 2, \mathrm{r}^{--}\right)$;

$\operatorname{plot}\left(\mathrm{x}, \mathrm{oF} 3, \mathrm{~g}^{--}\right)$;

title('Leg pendulum frequency'); grid on;

xlabel('Gait speed $\left.(\mathrm{m} / \mathrm{s})^{\prime}\right)$; ylabel('Pendulum frequency $\left.\left(\mathrm{s}^{\wedge}\{-1\}\right)^{\prime}\right)$;

legend('weighted average','unweighted average','lunar gravity estimate','drag-free estimate','physical data estimate','Location','SouthWest'); 
Appendix 5h: goal2.m

$\%$ Determine the impact of each factor (gravity, drag, and modelling $\%$ assumptions) on each metric. Take difference of metric functions between $\%$ adjacent elements of the test matrix, such that one of the elements is $\%$ adjacent to the true lunar gravity element.

clear all; close all; clc;

$\mathrm{x}=(0.5: 0.01: 1.5) ; \%$ speed, $\mathrm{m} / \mathrm{s}$

$\%$ Metric-estimating functions for each element of the text matrix

$\%$ Step length vs. gait speed

$\mathrm{sH}=0.3275{ }^{*} \mathrm{x}+0.2651 ; \mathrm{RsH}=0.6782 ;$

$\mathrm{sG}=0.2826 * \mathrm{x}+0.3995 ; \mathrm{RsG}=0.5285$;

$\mathrm{sE}=0.2766{ }^{*} \mathrm{x}+0.3846 ; \mathrm{RsE}=0.2503$;

$\mathrm{sD}=1.0149 . * \mathrm{x}-0.0566 ; \mathrm{RsD}=0.9918$;

$\mathrm{sB}=0.4647 . * \mathrm{x}+0.2828 ; \mathrm{RsB}=0.8675$;

$\mathrm{sA}=0.4514{ }^{*} \mathrm{x}+0.2886 ; \mathrm{RsA}=0.8796$

$\%$ Vertical torso displacement vs. gait speed

$\mathrm{tH}=0.0319 .{ }^{*} \mathrm{x}+0.0026 ; \mathrm{RtH}=0.6509 ;$

$\mathrm{tG}=0.0590{ }^{*} \mathrm{x}-0.0031 ; \mathrm{RtG}=0.4326$;

$\mathrm{tE}=-0.1858 * \mathrm{x}+0.1606 ; \mathrm{RtE}=0.5213$;

$\mathrm{tD}=0.1092{ }^{*} \mathrm{x}-0.0537 ; \mathrm{RtD}=0.9877$;

$\mathrm{tB}=-0.8430 . * \mathrm{x}+0.8515 ; \mathrm{RtB}=0.8760$;

$\mathrm{tA}=-0.8641 . * \mathrm{x}+0.8736 ; \operatorname{RtA}=0.8836$;

$\%$ Maximum leg angle vs. gait speed

$\mathrm{pH}=0.3509 .{ }^{*} \mathrm{x}+0.3071 ; \mathrm{RpH}=0.4101 ;$

$\mathrm{pG}=0.6842 *^{*} \mathrm{x}+0.1673 ; \mathrm{RpG}=0.5452$;

$\mathrm{pE}=0.0942 * \mathrm{x}+0.4832 ; \mathrm{RpE}=0.0132 ;$

$\mathrm{pD}=0.6310 . * \mathrm{x}-0.0697 ; \mathrm{RpD}=0.9870$;

$\mathrm{pB}=0.1283{ }^{*} \mathrm{x}+0.2619 ; \mathrm{RpB}=0.1754$;

$\mathrm{pA}=0.0644{ }^{*} \mathrm{x}+0.2980 ; \mathrm{RpA}=0.0264$;

$\%$ Leg pendulum frequency vs. gait speed

$\mathrm{oH}=2.2048{ }^{*} \mathrm{x}+1.9294 ; \mathrm{RoH}=0.4101 ;$

$\mathrm{oG}=4.2990 . * \mathrm{x}+1.0510 ; \mathrm{RoG}=0.5452 ;$

$\mathrm{oE}=0.5919 . * \mathrm{x}+3.0362 ; \mathrm{RoE}=0.0132 ;$

$\mathrm{oD}=3.9645 . * \mathrm{x}-0.4381 ; \mathrm{RoD}=0.9870$;

$\mathrm{oB}=0.7773{ }^{*} \mathrm{x}+0.3684 ; \mathrm{RoB}=0.8899 ;$

$\mathrm{oA}=0.4046{ }^{*} \mathrm{x}+1.8724 ; \operatorname{RoA}=0.0264$;

$\%$ Normalized differences and worst $\mathrm{R}^{\wedge} 2$ values of each pair of adjacent vertices $\mathrm{sEG}=(\mathrm{sE}-\mathrm{sG}) . / \mathrm{sG} ; \mathrm{RsEG}=\min ([\mathrm{RsE} \mathrm{RsG}])$;

$\mathrm{sBD}=(\mathrm{sB}-\mathrm{sD}) . / \mathrm{sD} ; \mathrm{RsBD}=\min ([\mathrm{RsB} \mathrm{RsD}])$;

$\mathrm{sBA}=(\mathrm{sB}-\mathrm{sA}) . / \mathrm{sA} ; \mathrm{RsBA}=\min ([\mathrm{RsB} \mathrm{RsA}])$;

$\mathrm{sHG}=(\mathrm{sH}-\mathrm{sG}) . / \mathrm{sG} ; \mathrm{RsHG}=\min ([\mathrm{RsH} \mathrm{RsG}])$;

$s E A=(s E-s A) . / s A ; \operatorname{RsEA}=\min ([\operatorname{RsE} \mathrm{RsA}])$;

$\mathrm{sHD}=(\mathrm{sH}-\mathrm{sD}) . / \mathrm{sD} ; \mathrm{RsHD}=\min ([\mathrm{RsH} \mathrm{RsD}])$;

$\mathrm{pEG}=(\mathrm{pE}-\mathrm{pG}) \cdot / \mathrm{pG} ; \mathrm{RpEG}=\min ([\mathrm{RpE} \mathrm{RpG}]) ;$

$\mathrm{pBD}=(\mathrm{pB}-\mathrm{pD}) \cdot / \mathrm{pD} ; \operatorname{RpBD}=\min ([\mathrm{RpB} R \mathrm{RD}]) ;$ 


$$
\begin{aligned}
& \mathrm{pBA}=(\mathrm{pB}-\mathrm{pA}) \cdot / \mathrm{pA} ; \mathrm{RpBA}=\min ([\mathrm{RpB} \mathrm{RpA}]) ; \\
& \mathrm{pHG}=(\mathrm{pH}-\mathrm{pG}) . / \mathrm{pG} ; \mathrm{RpHG}=\min ([\mathrm{RpH} \mathrm{RpG}]) ; \\
& \mathrm{pEA}=(\mathrm{pE}-\mathrm{pA}) \cdot / \mathrm{pA} ; \mathrm{RpEA}=\min ([\mathrm{RpE} \mathrm{RpA}]) ; \\
& \mathrm{pHD}=(\mathrm{pH}-\mathrm{pD}) . / \mathrm{pD} ; \mathrm{RpHD}=\min ([\mathrm{RpH} \mathrm{RpD}]) ; \\
& \mathrm{oEG}=(\mathrm{oE}-\mathrm{oG}) . / \mathrm{oG} ; \mathrm{RoEG}=\min ([\mathrm{RoE} \mathrm{RoG}]) ; \\
& \mathrm{oBD}=(\mathrm{oB}-\mathrm{oD}) . / \mathrm{oD} ; \mathrm{RoBD}=\min ([\mathrm{RoB} \operatorname{RoD}]) ; \\
& \mathrm{oBA}=(\mathrm{oB}-\mathrm{oA}) . / \mathrm{oA} ; \mathrm{RoBA}=\min ([\operatorname{RoB} \operatorname{RoA}]) ; \\
& \mathrm{oHG}=(\mathrm{oH}-\mathrm{oG}) \cdot / \mathrm{oG} ; \mathrm{RoHG}=\min ([\mathrm{RoH} \mathrm{RoG}]) ; \\
& \mathrm{oEA}=(\mathrm{oE}-\mathrm{oA}) \cdot / \mathrm{oA} ; \operatorname{RoEA}=\min ([\operatorname{RoE} \mathrm{RoA}]) ; \\
& \mathrm{oHD}=(\mathrm{oH}-\mathrm{oD}) . / \mathrm{oD} ; \mathrm{RoHD}=\min ([\mathrm{RoH} \mathrm{RoD}]) ;
\end{aligned}
$$

$\%$ Non-normalized due to values crossing zero

$\mathrm{tEG}=(\mathrm{tE}-\mathrm{tG}) ; \mathrm{RtEG}=\min ([\mathrm{RtE} \mathrm{RtG}]) ;$

$\mathrm{tBD}=(\mathrm{tB}-\mathrm{tD}) ; \mathrm{RtBD}=\min ([\mathrm{RtB} \mathrm{RtD}]) ;$

$\mathrm{tBA}=(\mathrm{tB}-\mathrm{tA}) ; \mathrm{RtBA}=\min ([\mathrm{RtB} \mathrm{RtA}]) ;$

$\mathrm{tHG}=(\mathrm{tH}-\mathrm{tG}) ; \mathrm{RtHG}=\min ([\mathrm{RtH} \mathrm{RtG}]) ;$

tEA $=($ tE - tA $) ;$ RtEA = min $([$ RtE RtA $]) ;$

$\mathrm{tHD}=(\mathrm{tH}-\mathrm{tD}) ; \mathrm{RtHD}=\min ([\mathrm{RtH} \mathrm{RtD}]) ;$

$\%$ Unweighted averages

$\mathrm{s} \_g r a v \_u=(\mathrm{sEG}+\mathrm{sBD}) . / 2$;

s_drag_u $=(\mathrm{sBA}+\mathrm{sHG}) \cdot / 2$;

s_phys_u $=($ sEA + sHD $) . / 2$;

$\mathrm{t} \_$grav_u $=(\mathrm{tEG}+\mathrm{tBD}) . / 2$;

t_drag_u $=($ tBA + tHG $) . / 2$;

t_phys_u $=(\mathrm{tEA}+\mathrm{tHD}) . / 2$;

p_grav_u $=(\mathrm{pEG}+\mathrm{pBD}) . / 2$;

p_drag_u $=(\mathrm{pBA}+\mathrm{pHG}) . / 2$;

p_phys_u $=(p E A+p H D) . / 2$;

o_grav_u $=(\mathrm{oEG}+\mathrm{oBD}) . / 2$;

o_drag_u $=(\mathrm{oBA}+\mathrm{oHG}) . / 2$;

o_phys_u $=($ oEA + oHD $) . / 2$;

$\%$ Weighted averages

s_grav_w $=($ sEG. $*$ RsEG + sBD. $*$ RsBD $) . /($ RsEG+RsBD $)$;

s_drag_w $=($ sBA. $*$ RsBA + sHG. $*$ RsHG $) . /($ RsBA+RsHG $)$;

s_phys_w $=($ sEA. $*$ RsEA + sHD. $*$ RsHD $) . /($ RsEA+RsHD $)$;

t_grav_w = ( tEG.*RtEG + tBD.*RtBD $). /(\mathrm{RtEG}+\mathrm{RtBD})$;

t_drag_w $=($ tBA.*RtBA + tHG.*RtHG $) . /(\mathrm{RtBA}+\mathrm{RtHG})$;

t_phys_w $=($ tEA. $*$ RtEA + tHD. $*$ RtHD $) . /(\mathrm{RtEA}+\mathrm{RtHD})$;

p_grav_w $=($ pEG. $* R p E G+$ pBD. $*$ RpBD $) . /(R p E G+R p B D) ;$

p_drag_w $=($ pBA. $*$ RpBA + pHG. $*$ RpHG $) . /(\mathrm{RpBA}+\mathrm{RpHG})$;

p_phys_w $=($ pEA. $*$ RpEA + pHD. $*$ RpHD $) . /(\mathrm{RpEA}+\mathrm{RpHD})$;

o_grav_w $=($ oEG. $*$ RoEG + oBD. $*$ RoBD $) . /($ RoEG+RoBD $)$;

o_drag_w $=($ oBA. $*$ RoBA + oHG. $*$ RoHG $) . /($ RoBA+RoHG $)$;

o_phys_w $=($ oEA. $*$ RoEA + oHD. $*$ RoHD $) . /($ RoEA+RoHD $)$; 


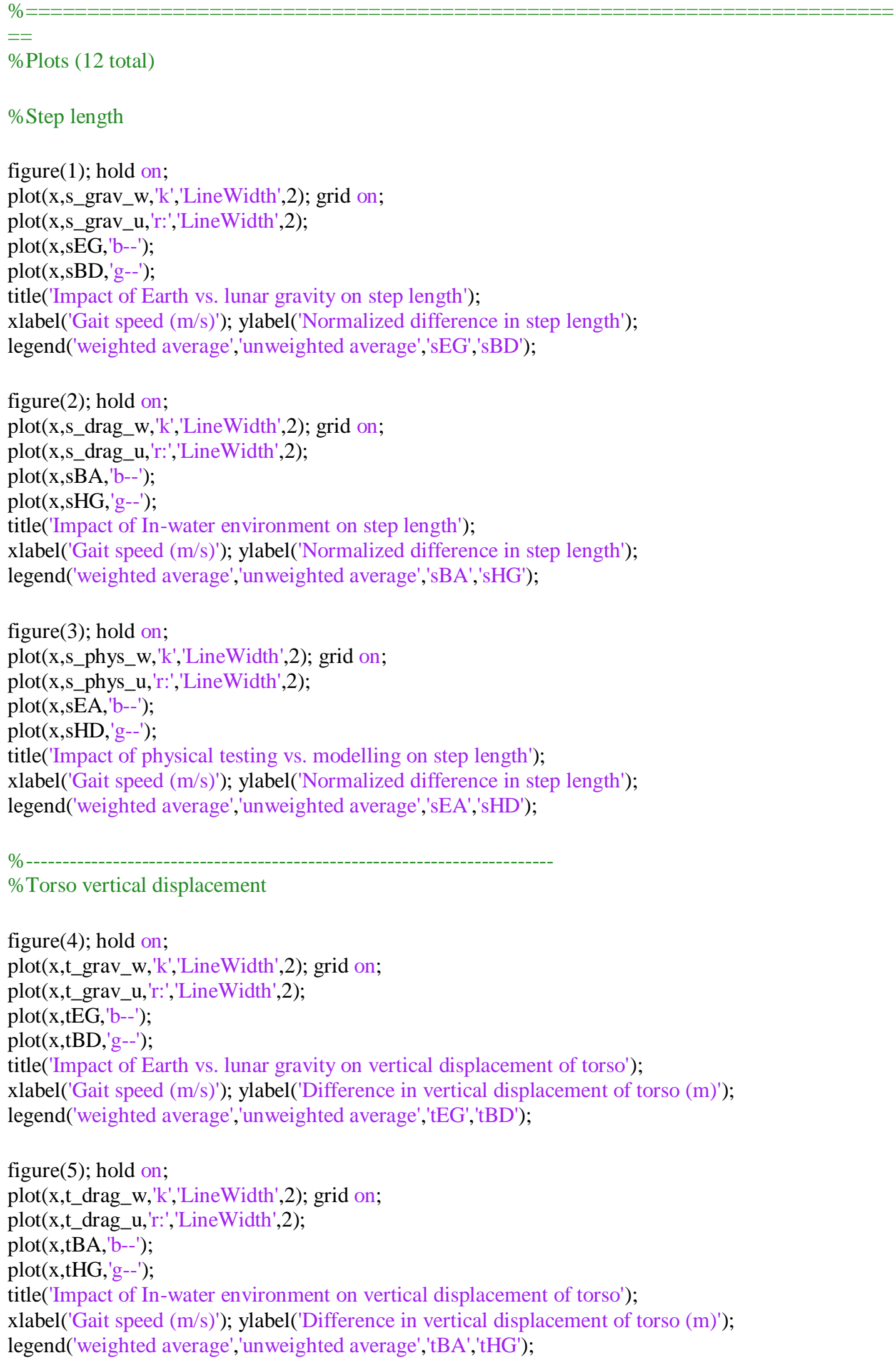




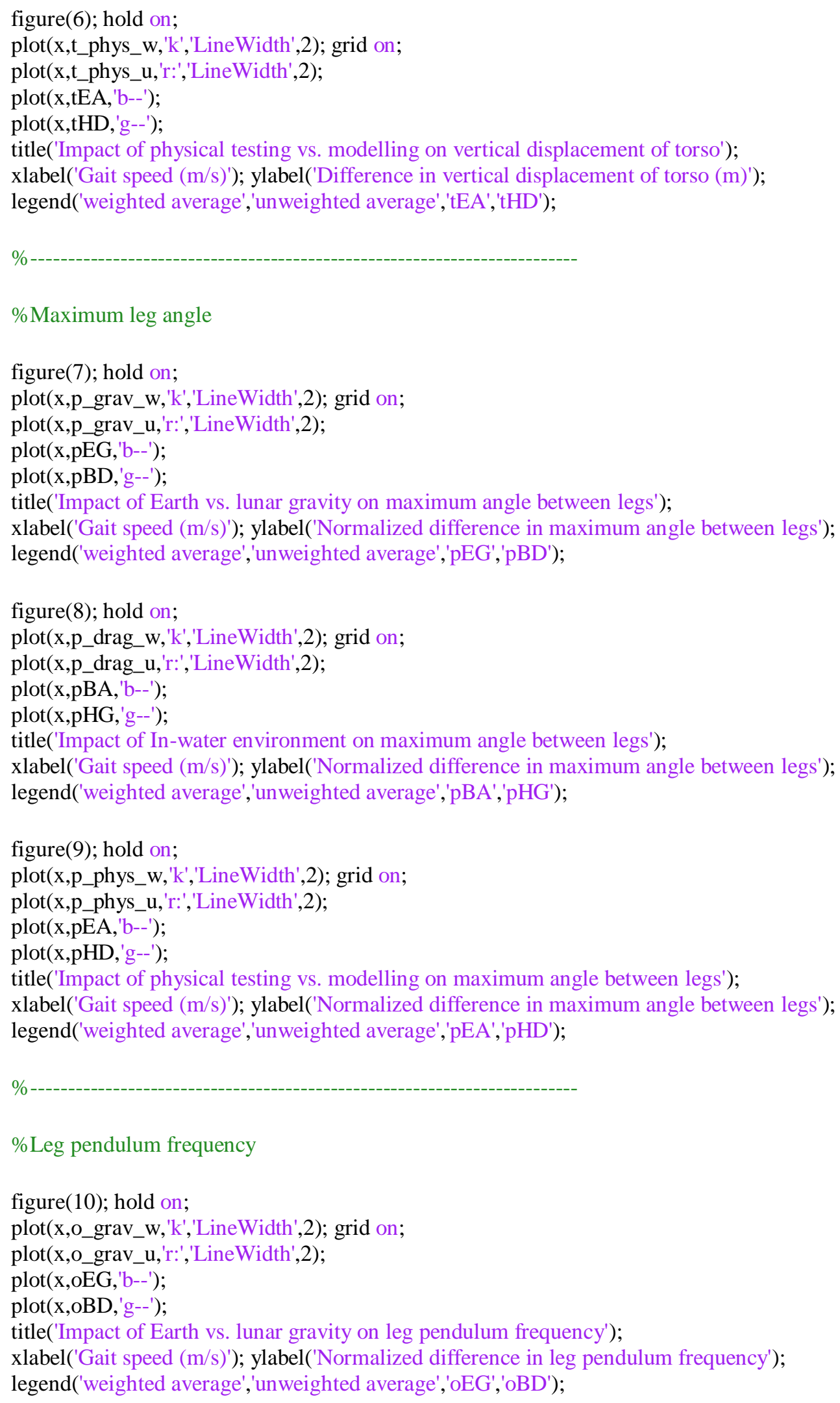


figure(11); hold on;

plot(x,o_drag_w,'k','LineWidth',2); grid on;

plot(x,o_drag_u,'r:','LineWidth',2);

$\operatorname{plot}\left(\mathrm{x}, \mathrm{oBA}, \mathrm{b}^{--}\right)$;

$\operatorname{plot}\left(\mathrm{x}, \mathrm{oHG}, \mathrm{g}-\mathrm{-}^{\prime}\right)$;

title('Impact of In-water environment on leg pendulum frequency');

xlabel('Gait speed $(\mathrm{m} / \mathrm{s})$ '); ylabel('Normalized difference in leg pendulum frequency');

legend('weighted average','unweighted average','oBA','oHG');

figure(12); hold on;

plot(x,o_phys_w,'k','LineWidth',2); grid on;

plot(x,o_phys_u,'r:','LineWidth',2);

$\operatorname{plot}(\mathrm{x}, \mathrm{oEA}, \mathrm{,b--})$;

$\operatorname{plot}\left(\mathrm{x}, \mathrm{oHD},{ }^{\prime} \mathrm{g}-\mathrm{-}^{\prime}\right)$;

title('Impact of physical testing vs. modelling on leg pendulum frequency');

xlabel('Gait speed $(\mathrm{m} / \mathrm{s})$ '); ylabel('Normalized difference in leg pendulum frequency');

legend('weighted average','unweighted average','oEA','oHD');

$\%$ 
Appendix 5i: tripleimport.m

clear all; close all; clc;

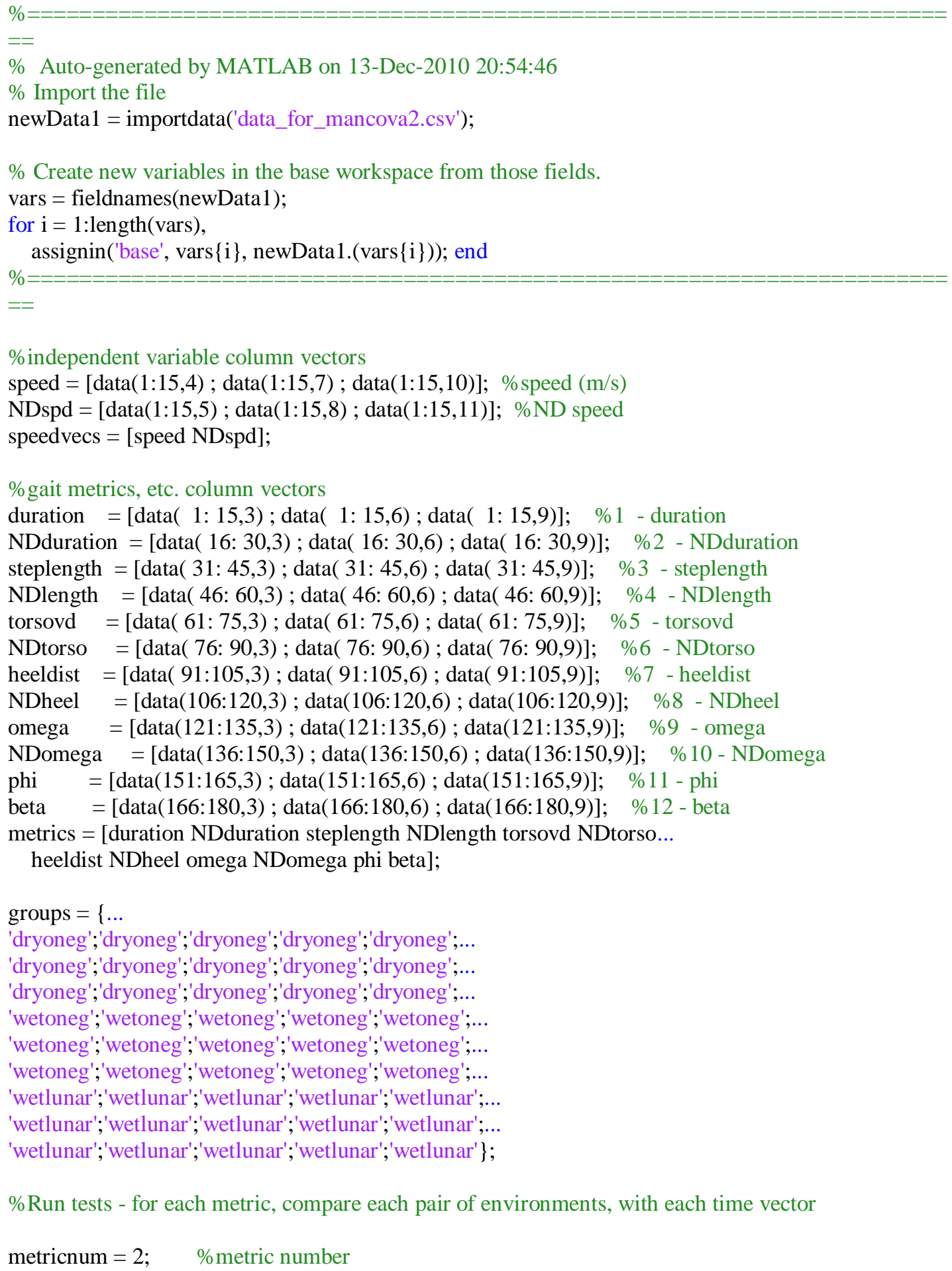




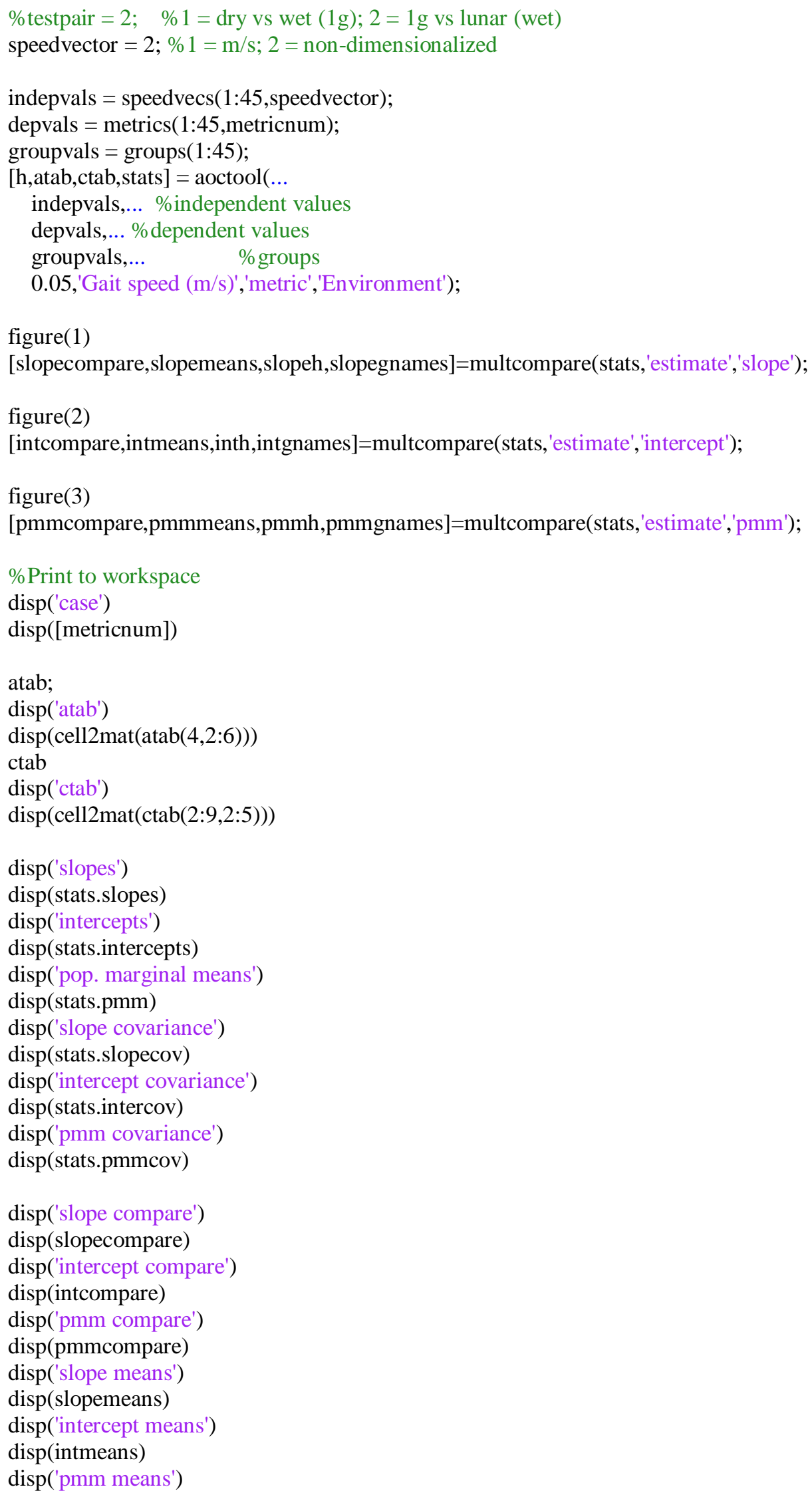


disp(pmmmeans) 


\section{Appendix 5j: triplebands.m}

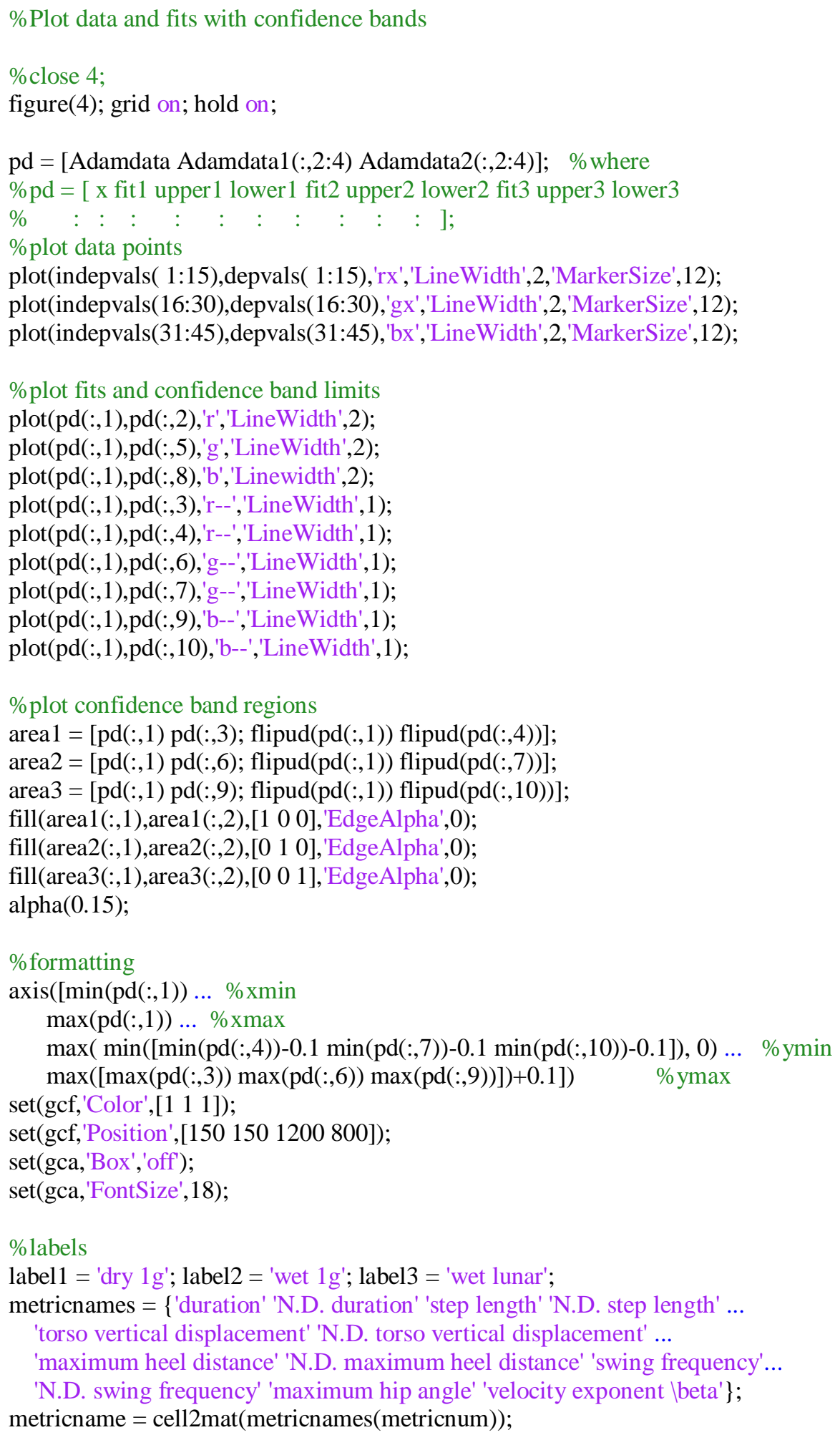


metriclabs $=\left\{\right.$ 'Duration $(\mathrm{s})^{\prime}$ 'N.D. duration' 'Step length (m)' ...

'N.D. step length' 'Torso vertical displacement (m)' ...

'N.D. torso vertical displacement' 'Maximum heel distance (m)' ...

'N.D. maximum heel distance' 'Swing frequency (Hz)' ...

'N.D. swing frequency' 'Maximum hip angle (radians)' ...

'Velocity exponent $\backslash$ beta' $\}$;

indeplabel $=\operatorname{cell} 2 \mathrm{mat}($ metriclabs $($ metricnum $))$;

xlabel('Non-dimensionalized gait speed:

speed/sqrt(\fontname $\{$ Times $\}\{$ litlbflg $\} \backslash$ fontname $\{$ Arial $\}$ )','FontWeight','bold','FontSize',20)

ylabel(indeplabel,'FontWeight','bold','FontSize',20)

title( \{'Linear fits and 95\% confidence bands for $\backslash \mathrm{bf'}$ [metricname ' Irmvs. N.D. gait

speed']\},'FontSize',24)

legend([label1 ' data'],[label2 ' data'],[label3 ' data'],[label1 ': lin. fit'],[label2 ': lin. fit'],[label3 ': lin.

fit'],'Location','EastOutside')

legend('boxoff') 


\section{Glossary}

\section{Cross-over:}

The point in a stride at which the feet exchange positions, i.e., the rear foot becomes the forward foot

\section{Heel-strike:}

The collision between foot and ground, treated in simplified passive dynamic walker models as perfectly inelastic

\section{Impulsive runner:}

A dynamic model of running in which contact with the ground is assumed to be an instantaneous, impulsive change in momentum

\section{Passive dynamic walker:}

A dynamic model of walking which achieves stable gaits without requiring active control

\section{Single-support:}

A portion of a gait cycle in which exactly one foot is in contact with, and transferring force to, the ground. In simplified passive dynamic walker models, the entire gait cycle is single support with the exception of two instantaneous transitions. Real walking gaits exhibit a period of dual support which makes up a small but finite portion of each stride. ${ }^{[18]}$

\section{Stance leg:}

A leg whose foot is in contact with the ground at a given moment

\section{Swing leg:}

A leg whose foot is not in contact with the ground at a given moment

\section{Toe-off:}

The instantaneous impulse applied to a foot at the moment it leaves contact with the ground. This impulse imparts the amount of energy lost due to inelastic heel-strike, plus any energy lost to non-conservative forces over the duration of the step. 


\section{Bibliography}

1. Alexander, R. and A.S. Jayes. A Dynamic Similarity Hypothesis for the Gaits of Quadrupedal Mammals. Journal of Zoology, v. 201, 1983. pp. 135-152.

2. Chang, Young-Hui, Hsuan-Wen Cathy Huang, Chris M. Hamerski, and Rodger Kram. The Independent Effects of Gravity and Inertia on Running Mechanics. The Journal of Experimental Biology, v. 203, 2000. pp. 229-238.

3. Donelan, J. Maxwell and Rodger Kram. The Effect of Reduced Gravity on the Kinematics of Human Walking: A Test of the Dynamic Similarity Hypothesis for Locomotion. The Journal of Experimental Biology, v. 200, 1997. pp. 3193-3201.

4. Donelan, J. Maxwell and Rodger Kram. Exploring Dynamic Similarity in Human Running Using Simulated Reduced Gravity. The Journal of Experimental Biology, v. 203, 2000. pp. 2405-2415.

5. Garcia, Mariano, Anindya Chatterjee, Andy Ruina, Michael Coleman. The Simplest Walking Model: Stability, Complexity, and Scaling. ASME Journal of Biomechanical Engineering. February 10, 1998.

6. Goldberg, J.H. and J.W. Alfred. Prediction of Physical Workload in Reduced Gravity. Aviation, Space, and Environmental Medicine, v. 59, 1988. pp. 11501157.

7. Koscielniak, Agnieszka. Development and Testing of a Metabolic Workload Measurement System for Space Suits. Digital Repository at the University of Maryland, June 5, 2007.

8. Kram, Rodger, Antoinette Domingo and Daniel P. Ferris. Effect of Reduced Gravity on the Preferred Walk-Run Transition Speed. The Journal of Experimental Biology, v. 200, 1997. pp. 821-826.

9. Kuo, Arthur D. Energetics of Actively Powered Locomotion Using the Simplest Walking Model. Journal of Biomechanical Engineering - Transactions of the ASME. (02002 ASME. February 2002, v.124. pp.113-120

10. Kuo, Arthur D. A Simple Model Predicts of Bipedal Walking Predicts the Preferred Speed-Step Length Relationship. Journal of Biomechanical Engineering - Transactions of the ASME. C2001 ASME. Vol. 123, June 2001. pp.264-269

11. Man-Systems Integration Standards. NASA. Volume I, Section 3 ANTHROPOMETRY AND BIOMECHANICS 3.2 General Anthropometrics \& 
Biomechanics Related Design Considerations

http://msis.jsc.nasa.gov/sections/section03.htm Last Updated: 5/7/08 2:04 PM

12. McGeer, T. Passive Dynamic Walking. May 1988. pp. 1-9.

13. McGeer, T. Chapter 4: Principles of Walking and Running. Advances in Comparative and Environmental Physiology, Vol 11. CSpringer-Verlag Berlin 1992. pp. 113-139.

14. Newman, Dava J., Harold L. Alexander, and Bruce W. Webbon. Energetics and Mechanics for Partial Gravity Locomotion. Aviation, Space, and Environmental Medicine, September 1994. V.65. pp. 815-823

15. Perusek, Gail P., John K. DeWitt, Carlos M. Grodsinsky, Kelly M. Gilkey, Peter R. Cavanagh. Zero Gravity Locomotion Simlators: New Ground-Based Analogs for Microgravity Exercise Simulation. Human Research Program Exercise Countermeasures Project. ISS and Human Research Office. ESA Symposium Technology for artificial gravity and microgravity simulation. ESTEC, Noorwijk, The Netherlands. Dec, 10-12, 2007.

16. Probe, John D. Quantitative Assessment of Human Motion Using Video Motion Analysis. Third Annual Workshop on Space Operations Automation and Robotics (SOAR 1989). pp. 155-157.

17. Srinivasan, Manoj and Andy Ruina. Computer optimization of a minimal biped model discovers walking and running. Nature. V.429, 5 January 2006. (02006 Nature Publishing Group. pp.72-75.

18. Whittle, Michael W. Gait Analysis: An Introduction. Fourth Ed. Butterworth Heinemann Elsevier. Edinburgh 2007. 
